\title{
Organocatalytic Asymmetric Reaction Cascade to Substituted Cyclohexylamines Highly
}

Jian Zhou and Benjamin List*

Max-Planck-Institut für Kohlenforschung, D-45470 Mülheim an der Ruhr, Germany

E-mail: list@mpi-muelheim.mpg.de

General: Reactions were monitored by thin layer chromatography using $0.25 \mathrm{~mm}$ E. Merck silica gel coated glass plates $(60 \mathrm{~F}-254)$ using UV light to visualize the course of reaction. Flash column chromatography was performed using E. Merck siliga gel (60, particle size $0.040-0.063 \mathrm{~mm}$ ). Chemical yields refer to pure isolated substances. $1 \mathrm{H}$ and $13 \mathrm{C} \mathrm{NMR}$ spectra were obtained using either a Bruker DPX-300 or AV-400 spectrometer. Chemical shifts are reported in ppm from tetramethylsilane with the solvent resonance as the internal standard. The following abbreviations were used to designate chemical shift mutiplicities: $\mathrm{s}=$ singlet, $\mathrm{d}=$ doublet, $\mathrm{t}=$ triplet, $\mathrm{q}=$ quartet, $\mathrm{h}=$ heptet, $\mathrm{m}=$ multiplet, $\mathrm{br}=$ broad. MS (EI): Finnigan MAT 8200 (70 eV), ESI-MS: Finnigan MAT 95, accurate mass determinations: Bruker APEX III FT-MS (7 T magnet).

Anhydrous Cyclohexane, MS $5 \AA$ powder, and all the Grignard reagents were purchased from Aldrich. MS $5 \AA$ powder was dried at $120^{\circ} \mathrm{C}$ under vacuum overnight before use. 3,4-Dihydro-6-methyl-2H-pyran-2-one was purchased from Aldrich and purified by column chromatography (pentane/ $\left.\mathrm{Et}_{2} \mathrm{O}, 10 / 1\right)$ before use. 1-Chloro-2-(chloromethyl)-3,5dioxahexane was purchased from TCI. 3,3'-bis(2,4,6-triisopropyl phenyl)-1,1'-binaphthyl2,2'-diyl hydrogen phosphate (TRIP) was synthesized according to literature report. ${ }^{1}$

\section{Substrate Synthesis}

Synthesis of substrates 1a, 1c, 1d, 1i, and $1 \mathrm{j}$ using a modification of a literature procedure $^{2}$.

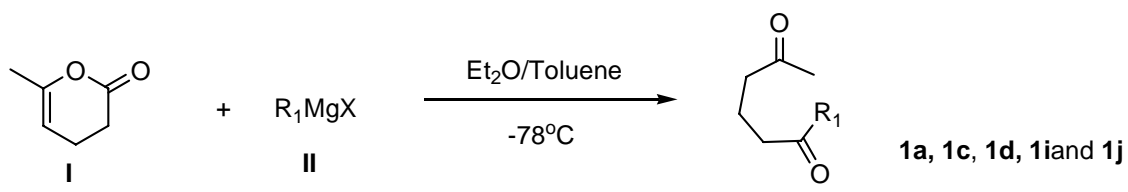

A solution of Grignard reagent II $(2.0 \mathrm{mmol})$ in toluene $(5.0 \mathrm{~mL})$ was slowly dropped into a vigorously stirred solution of 3,4-Dihydro-6-methyl-2H-pyran -2-one I (4.0 mmol) in $\mathrm{Et}_{2} \mathrm{O}(5.0 \mathrm{~mL})$ at $-78^{\circ} \mathrm{C}$ under argon. After the reaction was stirred at $-78^{\circ} \mathrm{C}$ for $8-10$ hours, $\mathrm{HCl}(1.0 \mathrm{M}, 10 \mathrm{~mL})$ was added at this temperature. The reaction mixture was warmed to room

(1) Seayad, J.; Seayad, A. M.; List, B. J. Am. Chem. Soc. 2006, 128, 1086.

(2) Harrowven, D. C.; Hannam, J. C. Tetrahedron 1999, 55, 9333. 
temperature, and then extracted with $\mathrm{Et}_{2} \mathrm{O}(2 \times 20 \mathrm{~mL})$. The combined organic phases were washed with water and brine, and then concentrated, followed by purification on a short $\mathrm{SiO}_{2}$ column (hexane or pentane/ $\mathrm{Et}_{2} \mathrm{O}, 10 / 1$ to $1 / 1$ ).

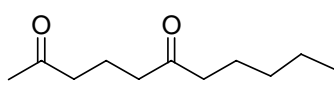

Diketone 1a was obtained as a colorless solid in $68 \%$ yield: ${ }^{1} \mathrm{H}$ NMR $\left(400 \mathrm{MHz}, \mathrm{CDCl}_{3}\right): \delta 0.8(\mathrm{t}, J=7.0 \mathrm{~Hz}, 3 \mathrm{H}), 1.25-1.32(\mathrm{~m}, 4 \mathrm{H}), 1.54-$ $1.58(\mathrm{~m}, 2 \mathrm{H}), 1.82-1.87(\mathrm{~m}, 2 \mathrm{H}), 2.13(\mathrm{~s}, 3 \mathrm{H}), 2.37(\mathrm{t}, J=7.4 \mathrm{~Hz}, 2 \mathrm{H}), 2.41-2.48(\mathrm{~m}, 4 \mathrm{H})$.<smiles>CC(=O)CCCC(=O)Cc1ccccc1</smiles>

Diketone 1c was obtained as a colorless solid in 53\% yield . ${ }^{1} \mathrm{H}$ NMR (400 $\left.\mathrm{MHz} \mathrm{CDCl}_{3}\right): \delta 1.77-1.85(\mathrm{~m}, 2 \mathrm{H}), 2.07(\mathrm{~s}, 3 \mathrm{H}), 2.40(\mathrm{t}, J=7.0 \mathrm{~Hz}, 2 \mathrm{H})$, $2.49(\mathrm{t}, J=7.4 \mathrm{~Hz}, 2 \mathrm{H}), 3.67(\mathrm{~s}, 2 \mathrm{H}), 7.18-7.20(\mathrm{~m}, 2 \mathrm{H}), 7.24-7.28(\mathrm{~m}, 1 \mathrm{H}), 7.31-7.35$ (m, 2H). ${ }^{13} \mathrm{C}$ NMR $\left(100 \mathrm{MHz}, \mathrm{CDCl}_{3}\right): \delta 17.62,29.85,40.66,42.33,50.17,127.05,128.76$, $129.39,134.13,207.86,208.35$.

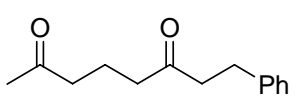

Diketone 1d was obtained as a colorless solid in $48 \%$ yield ${ }^{1} \mathrm{H}$ NMR (400 $\left.\mathrm{MHz}, \mathrm{CDCl}_{3}\right): \delta 1.78-1.85(\mathrm{~m}, 2 \mathrm{H}), 2.11(\mathrm{~s}, 3 \mathrm{H}), 2.40-2.44(\mathrm{~m}, 4 \mathrm{H}), 2.70-$ $2.73(\mathrm{~m}, 2 \mathrm{H}), 2.87-2.91(\mathrm{~m}, 2 \mathrm{H}), 7.16-7.20(\mathrm{~m}, 3 \mathrm{H}), 7.26-7.29(\mathrm{~m}, 2 \mathrm{H}) ;{ }^{13} \mathrm{C} \mathrm{NMR}(100 \mathrm{MHz}$, $\left.\mathrm{CDCl}_{3}\right): \delta 17.63,29.77,29.87,41.74,42.44,44.19,126.13,128.32,128.50,140.99,208.34$, 209.56. MS (EI): $218\left(\mathrm{M}^{+}, 52\right), 91(100), 105$ (74), 43 (73), 85 (38), 133 (34), 113 (26), 148 (6.7); HRMS (ESI): Exact mass calcd for $\mathrm{C}_{14} \mathrm{H}_{18} \mathrm{O}_{2} \mathrm{Na}_{1}[\mathrm{M}+\mathrm{Na}]^{+}$241.1199, Found: 241.1198.

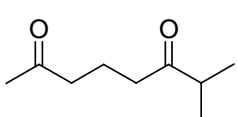

Diketone 1i was obtained as colorless oil in $41 \%$ yield. ${ }^{3}{ }^{1} \mathrm{H}$ NMR $(300 \mathrm{MHz}$, $\left.\mathrm{CDCl}_{3}\right): \delta 1.08(\mathrm{~d}, J=6.9 \mathrm{~Hz}, 6 \mathrm{H}), 1.83(\mathrm{~m}, 2 \mathrm{H}), 2.13(\mathrm{~s}, 3 \mathrm{H}), 2.44-2.53(\mathrm{~m}$, $4 \mathrm{H}), 2.56-2.63(\mathrm{~m}, 1 \mathrm{H})$.

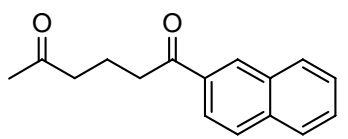

Diketone 1j was obtained as colorless solid in $42 \%$. ${ }^{1} \mathrm{H}$ NMR (400 $\left.\mathrm{MHz}, \mathrm{CDCl}_{3}\right): \delta 2.04-2.11(\mathrm{~m}, 2 \mathrm{H}), 2.17(\mathrm{~s}, 3 \mathrm{H}), 2.61(\mathrm{t}, J=7.0 \mathrm{~Hz}$, 2H), 3.15 (t, $J=7.4 \mathrm{~Hz}, 2 \mathrm{H}), 7.53-7.62(\mathrm{~m}, 2 \mathrm{H}), 7.88(\mathrm{t}, J=8.3,7.4 \mathrm{~Hz}, 2 \mathrm{H}), 7.97(\mathrm{~d}, J=8.0$ $\mathrm{Hz}, 1 \mathrm{H}), 8.03$ (q, $J=4.3,1.6 \mathrm{~Hz}, 1 \mathrm{H}), 8.48(\mathrm{~s}, 1 \mathrm{H}) .{ }^{13} \mathrm{C} \mathrm{NMR}\left(100 \mathrm{MHz}, \mathrm{CDCl}_{3}\right): \delta 18.01$, 29.60, 37.11, 42.28, 123.44, 126.41, 127.40, 128.09, 129.22, 129.40, 132.18, 133.77, 135.23, 199.35, 208.21; MS (EI): $240\left(\mathrm{M}^{+}, 21\right), 155(100), 127$ (53), 170 (16), 43 (10), 77 (5), 197 (5); HRMS (ESI): Exact mass calcd for $\mathrm{C}_{16} \mathrm{H}_{16} \mathrm{O}_{2} \mathrm{Na}_{1}[\mathrm{M}+\mathrm{Na}]^{+}$263.1042, Found: 263.1042.

(3) Our method is more convenient than the literature method: (a) Jun J.-G.; Shin, H. S.; Kim, S. H. J. Chem. Soc. Perkin Trans. 1. 1993, 1815. (b) Jun J.-G.; Mundy, B. P. Bull. Korean Chem. Soc. 1988, 9, 135. 
Substrates 1b, 1f, $1 \mathbf{g}$ and $\mathbf{1 h}$ were synthesized according to a known procedure from 2,6lutidine: ${ }^{4}$
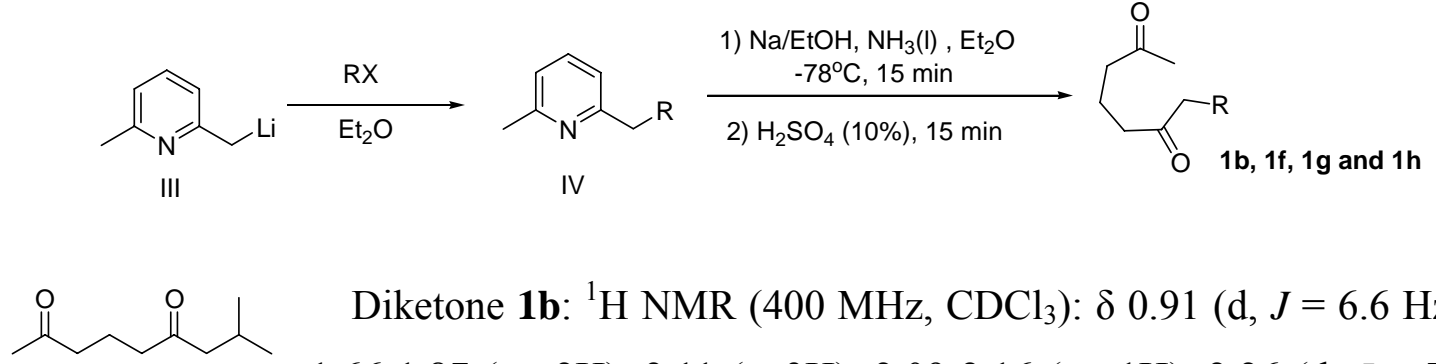

Diketone 1b: ${ }^{1} \mathrm{H}$ NMR (400 MHz, $\left.\mathrm{CDCl}_{3}\right): \delta 0.91(\mathrm{~d}, J=6.6 \mathrm{~Hz}, 6 \mathrm{H})$, 1.66-1.87 (m, 2H), $2.11(\mathrm{~s}, 3 \mathrm{H}), 2.09-2.16(\mathrm{~m}, 1 \mathrm{H}), 2.26(\mathrm{~d}, J=7.0 \mathrm{~Hz}$, $2 \mathrm{H}), 2.39-2.47(\mathrm{~m}, 4 \mathrm{H})$.

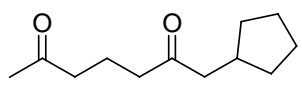

Diketone 1f: ${ }^{1} \mathrm{H}$ NMR (400 MHz, $\left.\mathrm{CDCl}_{3}\right): \delta$ 1.05-1.08 (m, 2H), 1.52$1.60(\mathrm{~m}, 4 \mathrm{H}), 1.79-1.85(\mathrm{~m}, 4 \mathrm{H}), 2.13(\mathrm{~s}, 3 \mathrm{H}), 2.15-2.25(\mathrm{~m}, 1 \mathrm{H}), 2.40-$ $2.48(\mathrm{~m}, 6 \mathrm{H}) ;{ }^{13} \mathrm{C} \mathrm{NMR}\left(100 \mathrm{MHz}, \mathrm{CDCl}_{3}\right): \delta 17.73,24.95,29.89,32.61,35.63,41.70,42.58$, 49.14, 208.48, 210.63. MS (EI): 196 (M+2, 2), 43 (100), 128 (89), 83 (84), 111 (44), 95 (26), 71 (52), 58 (18), 139 (3); HRMS (ESI): Exact mass calcd for $\mathrm{C}_{12} \mathrm{H}_{20} \mathrm{O}_{2} \mathrm{Na}_{1}[\mathrm{M}+\mathrm{Na}]^{+}$219.135552, Found: 219.135392.

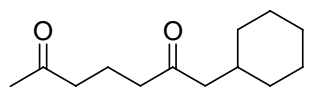

Diketone 1g: ${ }^{1} \mathrm{H}$ NMR $\left(300 \mathrm{MHz}, \mathrm{CDCl}_{3}\right): \delta$ 0.85-0.93 $(\mathrm{m}, 2 \mathrm{H})$, 1.05-1.32 (m, 3H), 1.58-1.69 (m, 6H), 1.78-1.87 (m, 2H), $2.13(\mathrm{~s}, 3 \mathrm{H})$, $2.25(\mathrm{~d}, J=6.9 \mathrm{~Hz}, 2 \mathrm{H}), 2.39-2.48(\mathrm{~m}, 4 \mathrm{H})$.

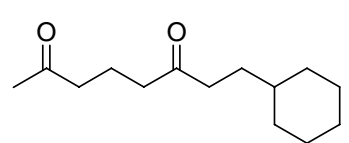

Diketone 1h: ${ }^{1} \mathrm{H}$ NMR (300 MHz, $\left.\mathrm{CDCl}_{3}\right): \delta$ 0.85-0.92 (m, 2H), $1.08-1.26(\mathrm{~m}, 4 \mathrm{H}), 1.46(\mathrm{q}, J=3.9,4.5 \mathrm{~Hz}, 2 \mathrm{H}), 1.59-1.71(\mathrm{~m}, 5 \mathrm{H})$, 1.83 (h, $J=7.2 \mathrm{~Hz}, 2 \mathrm{H}), 2.13$ (s, 3H), 2.39-2.48 (m, 6H).

Diketone 1e was prepared according to a known procedure ${ }^{5}{ }^{1} \mathrm{H}$ NMR $\left(400 \mathrm{MHz}, \mathrm{CDCl}_{3}\right): \delta$ $1.84(\mathrm{~h}, J=7.1 \mathrm{~Hz}, 2 \mathrm{H}), 2.13(\mathrm{~s}, 6 \mathrm{H}), 2.47$ (t, $J=7.1 \mathrm{~Hz}, 4 \mathrm{H})$.
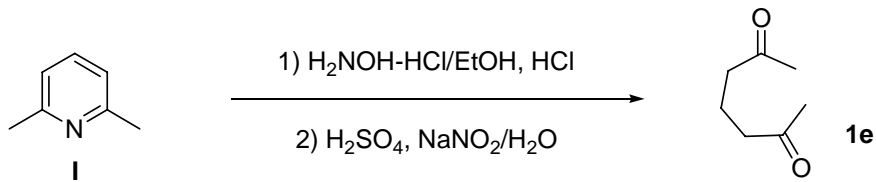

(4) Danishefsky, B.; Zimmer, A. J. Org. Chem. 1976, 41, 4059.

(5) Overberger, C. G.; Gibb, Jr. T. B.; Chibnik, S.; Huang, P. T.; Monagle, J. J. J. Am. Chem. Soc. 1952, 74, 3290. 
Diketone 1k was prepared according to a known procedure ${ }^{6}$ from 1-Chloro-2-(chloromethyl)3,5-dioxahexane VI. ${ }^{1} \mathrm{H}$ NMR $\left(300 \mathrm{MHz}, \mathrm{CDCl}_{3}\right): \delta 2.18(\mathrm{~s}, 3 \mathrm{H}), 4.17$ (s, 2H).

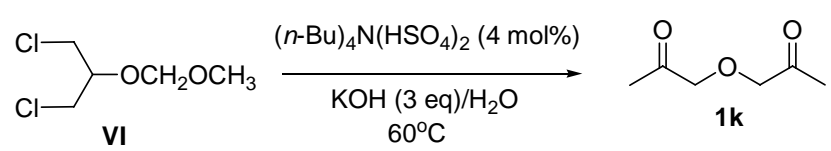

Diketone 11 was prepared according to a known procedure ${ }^{7}$ from 1-chloropropan-2-one VII. ${ }^{1} \mathrm{H}$ NMR (300 MHz, $\mathrm{CDCl}_{3}$ ): $\delta 2.27$ (s, 3H), 3.31 (s, 2H).

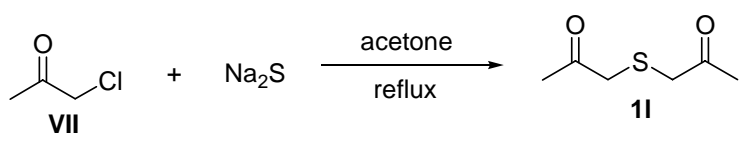

\section{Assignment of the Relative Configuration}

Although compound 4e-PMP is known, ${ }^{8}$ its relative configuration has not been assigned by now. A NMR study was carried out to assign the relative configuration.

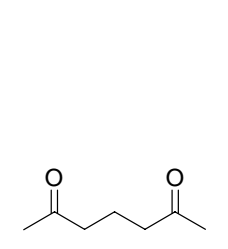

$1 \mathrm{e}$
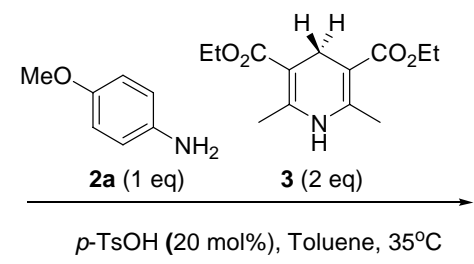

$72 \%$

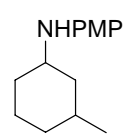

4e-PMP

$\operatorname{trans} / c i s=3 / 1$

By ${ }^{1} \mathrm{H}$ NMR, ${ }^{13} \mathrm{C}$ NMR, DEPT $\left(\mathrm{CH}, \mathrm{CH}_{3}\right.$ positive, $\mathrm{CH}_{2}$ negative),

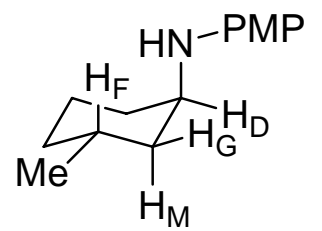

trans-4e-PMP
$\mathrm{C}-\mathrm{H}-\mathrm{COSY}$ and $\mathrm{H}-\mathrm{H}-\mathrm{COSY}$, the major product in the $p-\mathrm{TsOH}$ catalyzed reaction was determined to be the trans product, with the $\mathrm{N}$ moiety is on the axial position and methyl group on the equatorial position.

(6) Gu, X.-P.; Ikeda, I.; Komada, S.; Masuyama, A.; Okahara, M. J. Org. Chem. 1986, 51, 5425.

(7) Böhme, H.; Pfeifer, H.; Schneider, E. Chem. Ber. 1942, 75, 900.

(8) De Savignac; M. A.; Bon, M. M.; Mazarguil, H.; Latters, A. Bull. Soc. Chim. Fran. 1975, 2057-2065. 


\begin{tabular}{|c|c|c|}
\hline Carbon & Chemical shift & $\mathrm{H}$ \\
\hline a & $151.77 \mathrm{~s}$ & - \\
\hline$b$ & $141.64 \mathrm{~s}$ & - \\
\hline c & $114.96 \mathrm{~d}$ & A \\
\hline$d$ & $114.64 \mathrm{~d}$ & B \\
\hline e & $55.84 \quad q$ & C \\
\hline$f$ & $48.55 \mathrm{~d}$ & $\mathrm{D}$ \\
\hline g & 38.92 & $G, M$ \\
\hline $\mathrm{h}$ & 33.98 & $\mathrm{IH}, \mathrm{N}$ \\
\hline i & 30.49 & $\mathrm{HI}, \mathrm{J}$ \\
\hline j & 27.13 & $\mathrm{~F}$ \\
\hline $\mathrm{k}$ & 21.72 & $\mathrm{O}$ \\
\hline 1 & 20.52 & $\mathrm{~K}, \mathrm{~L}$ \\
\hline
\end{tabular}

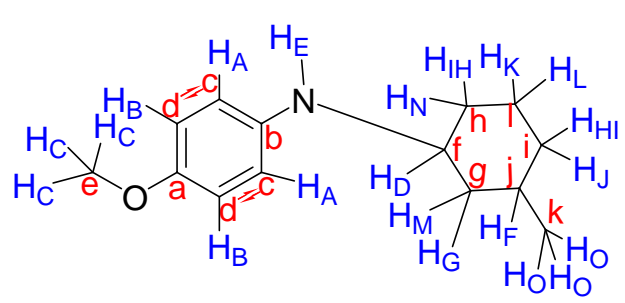

\begin{tabular}{|c|c|c|c|c|}
\hline \multirow{2}{*}{$\begin{array}{l}\text { Proton } \\
\text { A }\end{array}$} & \multicolumn{2}{|c|}{ Chemical shift } & \multirow{2}{*}{$\frac{[\mathrm{H}]}{2}$} & \multirow{2}{*}{$\begin{array}{l}\text { cosy } \\
\text { B }\end{array}$} \\
\hline & 6.76 & d & & \\
\hline B & 6.57 & d & 2 & $A$ \\
\hline $\mathrm{C}$ & 3.73 & s & 3 & - \\
\hline D & 3.58 & & 1 & $\mathrm{FE}, \mathrm{HI}, \mathrm{M}$ \\
\hline$E$ & ca.3.4 & br. & 1 & - \\
\hline $\mathrm{F}$ & 1.73 & & 1 & $\mathrm{O}, \mathrm{G}$ \\
\hline G & 1.69 & & 1 & $\mathrm{M}, \mathrm{F}$ \\
\hline $\mathrm{H}$ & 1.64 & & 1 & $\mathrm{~N}$ \\
\hline I & 1.61 & & 1 & $\mathrm{~N}$ \\
\hline $\mathrm{J}$ & 1.56 & & 1 & $\mathrm{KL},(\mathrm{N})$ \\
\hline K & 1.53 & & 1 & $\mathrm{~N}, \mathrm{~L}$ \\
\hline $\mathrm{L}$ & 1.50 & & 1 & $\mathrm{~K}, \mathrm{~N}$ \\
\hline M & 1.33 & $t(d, d)$ & 1 & $\mathrm{~F}$ \\
\hline$N$ & 1.04 & & 1 & $\mathrm{HI}$ \\
\hline $\mathrm{O}$ & 0.90 & $d$ & 3 & FG \\
\hline
\end{tabular}

The red color indicates the carbon, while the blue color the proton. The differentiation of carbon $\mathrm{c}$ and $\mathrm{d}$, proton $\mathrm{H}_{\mathrm{H}}$ and $\mathrm{H}_{\mathrm{f}}$ failed.

The assignment was based on the following facts:

1. The chemical shift of the signals at $\delta_{\mathrm{C}}=20.5 \mathrm{ppm}(\mathrm{t})$ indicates that if the cyclohexane ring has a chair conformation, then one of the subsitituents in $\gamma$-position must be axial. The assignment was based on the following facts:

2. The chemical shift of $\mathrm{C}_{\mathrm{j}}(27.1 \mathrm{ppm})$ is exactly what would be expected if the methyl group were axial. However, the ${ }^{13} \mathrm{C}$ chemical shift of the methyl substituent itself (21.7 ppm) is more consistent with this group being equatorial (expected shift ca. 22.8-23.5 ppm). The signal of an axial methyl group would be expected to be observed at ca. $17.5 \mathrm{ppm}$. Therefore, the high field shifts of $C_{j}(27.1 \mathrm{ppm})$ and $C_{1}(20.5 \mathrm{ppm})$ must be due to the NHPMP substituent in $\gamma$-position being axial.

3. A full assignment of the proton NMR spectrum was difficult because of severe crowding in the range 1.75 to $1.47 \mathrm{ppm}$. However, examination of the multiplet at $1.33 \mathrm{ppm}$ (signal M, $\mathrm{H}_{\mathrm{M}}$, axial) does show that the $1 \mathrm{H}, 1 \mathrm{H}$ couplings are consistent with the above analysis: ca. $13.5 \mathrm{~Hz} \quad{ }^{2} \mathrm{~J}$ (H-7ax, H-7eq)

ca. $10.6 \mathrm{~Hz} \quad{ }^{3} \mathrm{~J}(\mathrm{H}, \mathrm{H})$, trans coupling to an axial proton) and ca. $3.9 \mathrm{~Hz} \quad{ }^{3} \mathrm{~J}(\mathrm{H}, \mathrm{H})$, gauche coupling to an equatorial proton.

Both of the NMR data of trans and cis product 4e-PMP were attached at the end. As can be seen, the chemical shift of $\mathrm{H}_{\mathrm{D}}(3.58 \mathrm{ppm})$ in this trans diastereomer is in lower field than that of $\mathrm{H}_{\mathrm{D}}(3.15 \mathrm{ppm})$ in this cis diastereomer. This characteristic chemical shift difference was used to assign cis and trans configuration of all products. 


\section{Reaction Optimization}

\section{I) Phosphoric Acids}

Commercially available 3-methylcyclohexenone was subjected to the model reaction, using $10 \mathrm{~mol} \%$ of chiral acid, 1.5 equivalents of $\mathrm{PMP}^{-\mathrm{NH}_{2}}$ 2a, and 2.2 equivalents of Hantzsch ester in toluene at $50^{\circ} \mathrm{C}$. Of the studied phosphoric acids, TRIP turned out to be the most reactive and enantioselective catalyst (entry 6).

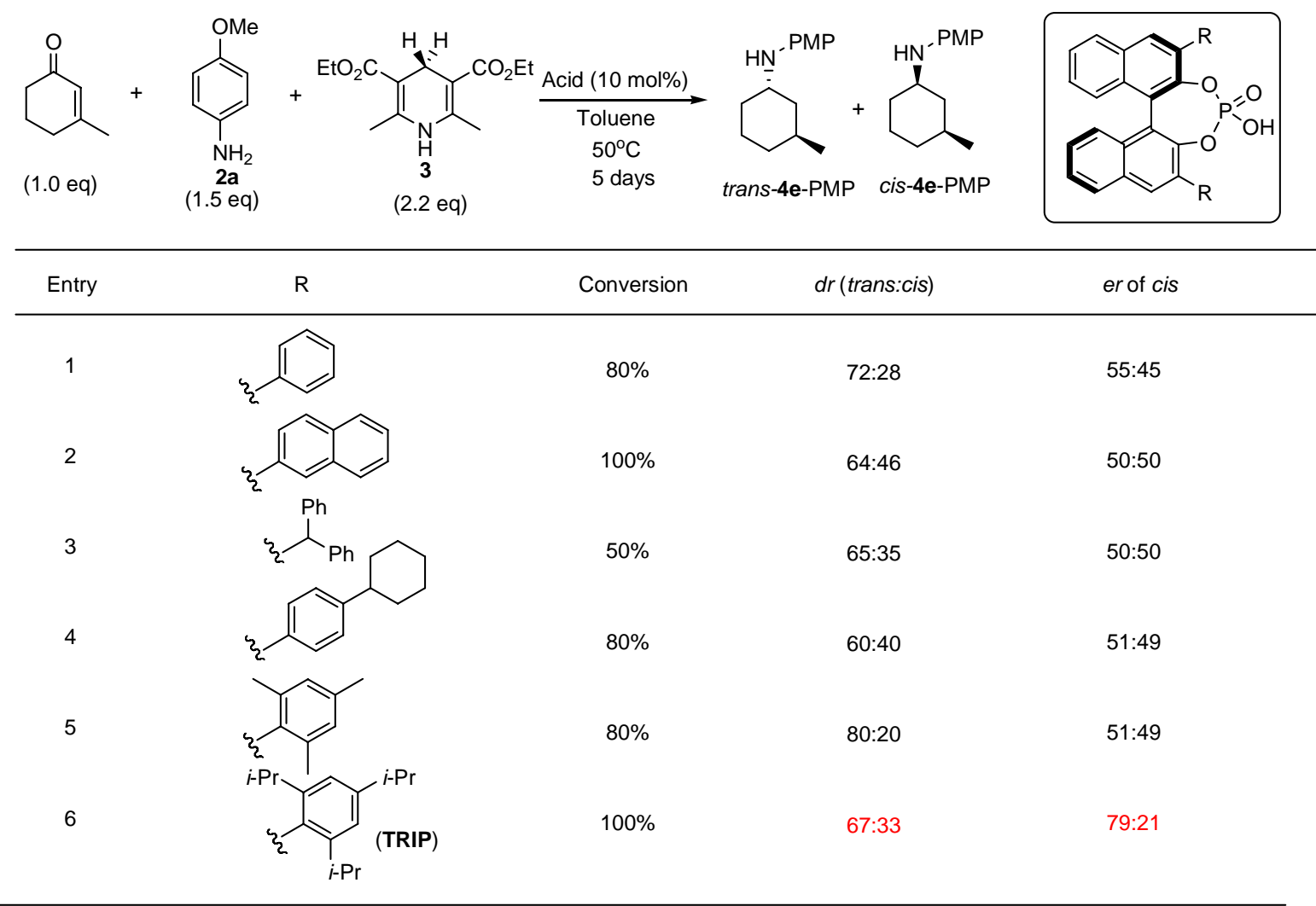

Note: Cis/Trans dertermined by ${ }^{1} \mathrm{H}$ NMR and er by HPLC

\section{II) Additives}<smiles>CC1=CC(=O)CCC1</smiles>

(1.0 eq)<smiles>COc1ccc(N)cc1</smiles>

$2 \mathrm{a}$<smiles>CCOC(=O)c1[cH+]c(C(=O)OCC)c(C)[nH]c1C</smiles>$$
(2.2 \mathrm{eq})
$$

$\underset{\text { Toluene }}{\stackrel{\text { TRIP }(10 \mathrm{~mol} \%)}{\longrightarrow}}$ $50^{\circ} \mathrm{C}$

trans-4e-PMP Without MS 5Å: With MS $5 \AA$ :
3 days

1 day full conversion er of cis: $79: 21$

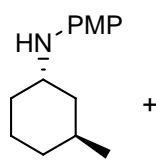
er of cis: $87: 13$

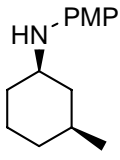

cis-4e-PMP 
MS $5 \AA$ has a strong effect on this reaction. Both reactivity and enantioselectivity greatly improved, and the diastereoselectivity reversed. In the presence of MS $5 \AA$, the reaction was complete within one day, the trans/cis ratio reversed from $67 / 33$ to $24 / 76$, and the er of the cis product improved from 79:21 to 87:13.

It should be noted that TRIP in combination with MS $5 \AA$ is crucial for the high cis selectivity in the reductive amination step, supported by the following control experiments employing (R)-3-methylcyclo-hexanone 11e. With $\mathrm{TsOH}$ as the catalyst, product 4e was obtained with trans selectivity (Condition A). The "matched" case (condition B) and "mismatched" case (Condtion C) further confirmed the importance of TRIP to secure cis selectivity. Furthermore, two typical reductive amination methods (condition D and E) only afforded the product with trans-selectivity, which demonstrated it difficult to obtain high cisselectivity by reductive amination of chiral 3-substituted cyclohexanone $\mathbf{1 1 .}$

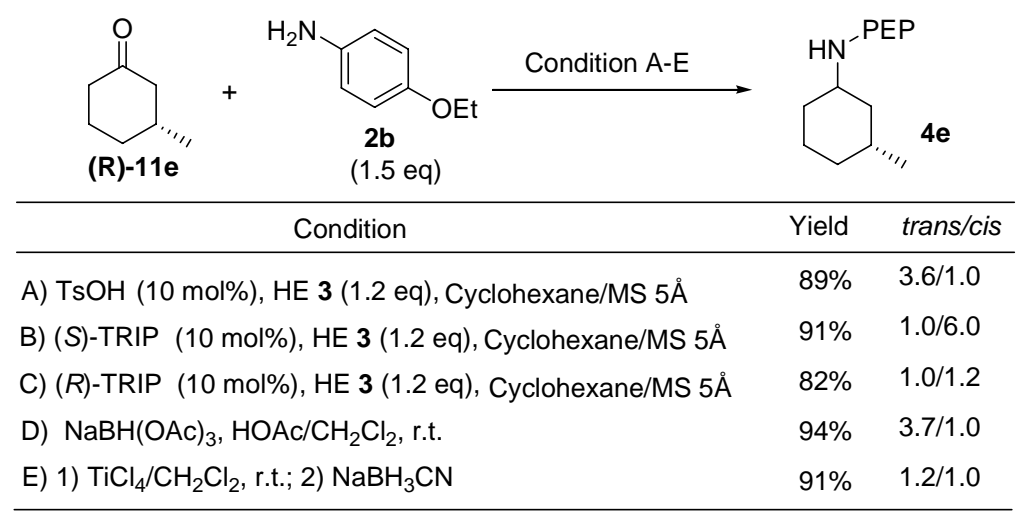




\section{III) Solvents}

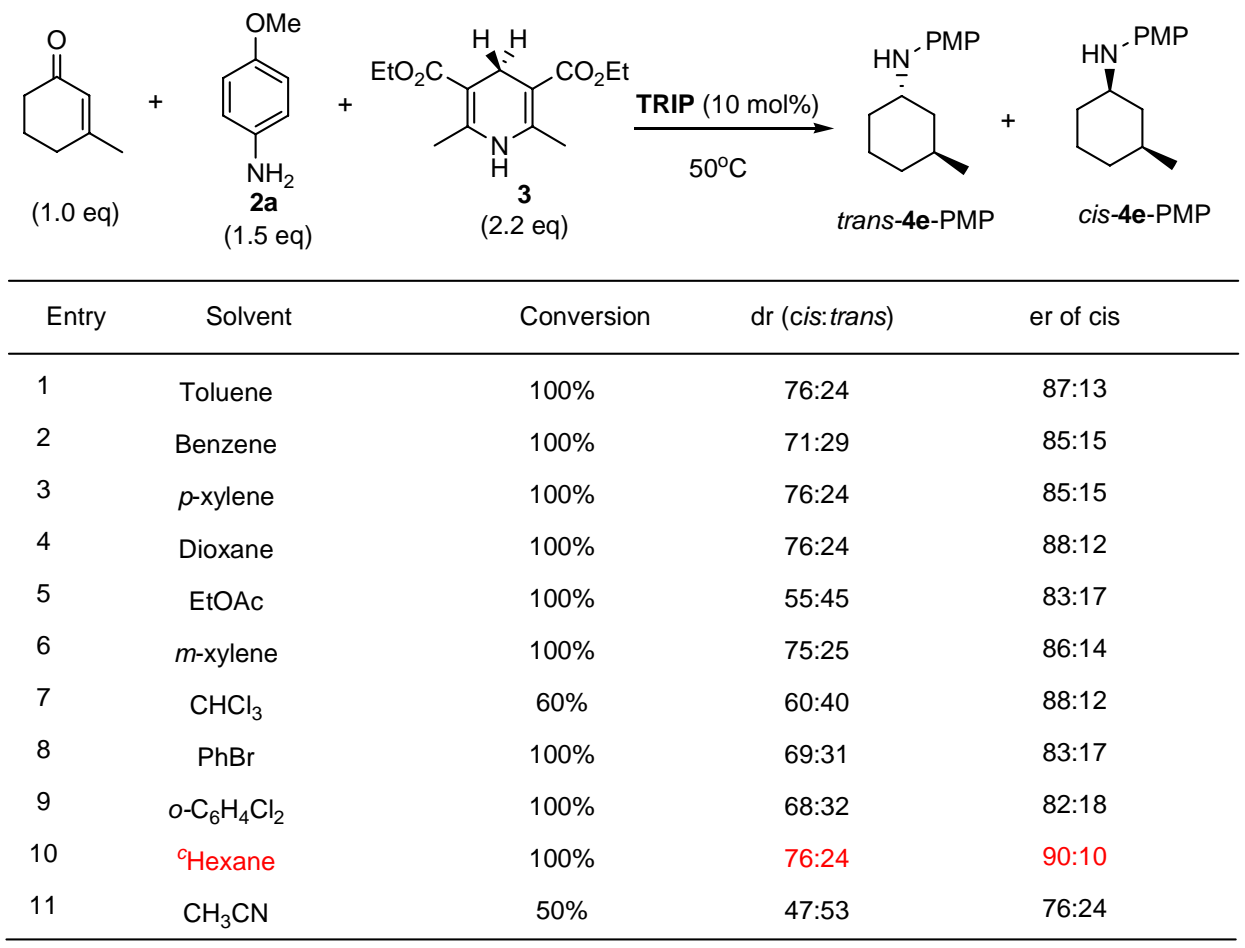

Note: Cis/Trans dertermined by ${ }^{1} \mathrm{H}$ NMR and Ee\% by HPLC

Of the solvents studied, cyclohexane afforded the highest selectivity (entry 10).

\section{IV) Amines}

Several commercially available aniline derivatives were employed to improve the stereoselectivity. Generally speaking, p-alkoxy-substituted anilines afforded much better stereoselectivity than ortho-substituted ones. p-Ethoxyphenylamine gave the highest diastereoselectivity and enantioselectivity (entry 2 ). 


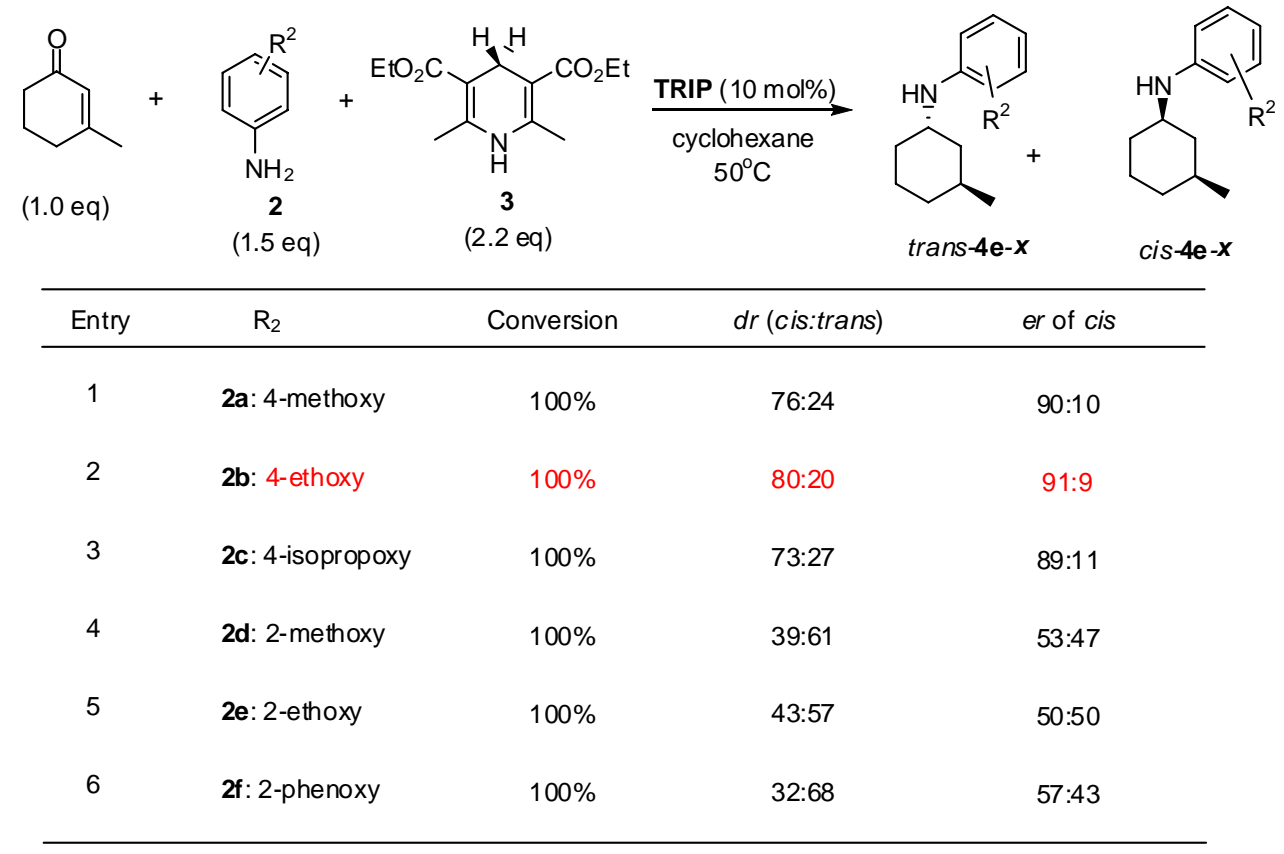

Note: Cis/T rans dertermined by GC-MS or ${ }^{1} \mathrm{H}$ NMR and er by HPLC

\section{General Procedure for the Organocatalytic Cascade Reaction}

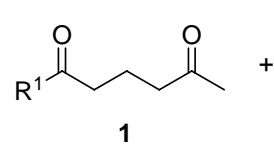

(1.0 eq)<smiles>[R]Oc1ccc(N)cc1</smiles>

(1.5 eq)<smiles>CCOC(=O)C1=C(C)NC(C)=C(C(=O)OCC)C1</smiles>

(2.2 eq)

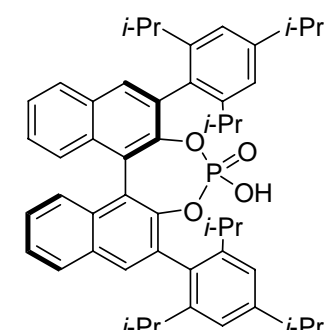

(R)-TRIP (10 mol\%)

Cyclohexane/MS $5 \AA$ $50^{\circ} \mathrm{C}$

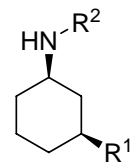

cis-4

To a Schlenk tube was added 2,6-dione 1 (0.25 mmol), p-ethoxyphenyl amine $(0.38 \mathrm{mmol})$, Hantzsch ester 3 (0.55 mmol) and TRIP (19 mg, $0.025 \mathrm{mmol})$, and then charged with argon, followed by adding MS $5 \AA(250 \mathrm{mg})$ and anhydrous cyclohexane $(2.5 \mathrm{~mL})$. The mixture was stirred at $50^{\circ} \mathrm{C}$ for 72 hours. To determine the ratio of trans to cis product, $20 \mu \mathrm{L}$ of reaction mixture was taken for GC-MS analysis, or $0.5 \mathrm{~mL}$ of crude mixture for ${ }^{1} \mathrm{H}$ NMR analysis. And then the sample for analysis and the rest of the product were recombined for column chromatographic purification using the indicated solvent mixtures. 


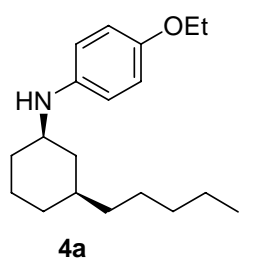

Column chromatography (3\% EtOAc in hexane) afforded the desired product 4a in 75\% yield as colorless oil. HPLC analysis (Chiralcel AD-H, 4\% ${ }^{\mathrm{i}} \mathrm{PrOH} /$ heptane, $1.0 \mathrm{~mL} / \mathrm{min}, 247 \mathrm{~nm}$; $\mathrm{t}_{\mathrm{r}}($ major $)=6.68 \mathrm{~min}, \mathrm{t}_{\mathrm{r}}($ minor $)=7.52$ min) gave the isomeric composition of the product: er $=95 \cdot 3: 4.7$. ${ }^{1} \mathrm{H}$ NMR $\left(400 \mathrm{MHz}, \mathrm{CDCl}_{3}\right): \delta 0.70(\mathrm{q}, J=12.0 \mathrm{~Hz}, 1 \mathrm{H}), 0.76-0.87(\mathrm{~m}, 1 \mathrm{H}), 0.88(\mathrm{t}, J$ $=7.2 \mathrm{~Hz}, 3 \mathrm{H}), 0.91-1.00(\mathrm{~m}, 1 \mathrm{H}), 1.17-1.40(\mathrm{~m}, 10 \mathrm{H}), 1.36(\mathrm{t}, J=7.0 \mathrm{~Hz}, 3 \mathrm{H}), 1.71-1.81(\mathrm{~m}$, 2H), 2.09-2.13 (m, 2H), 2.5-3.0 (br, 1H), 3.13 (tt, $J=11.2,3.6 \mathrm{~Hz}, 1 \mathrm{H}), 3.95$ (q, $J=7.0 \mathrm{~Hz}$, $2 \mathrm{H}), 6.52-6.57(\mathrm{~m}, 2 \mathrm{H}), 6.73-6.77(\mathrm{~m}, 2 \mathrm{H}) .{ }^{13} \mathrm{C} \mathrm{NMR}\left(100 \mathrm{MHz}, \mathrm{CDCl}_{3}\right): \delta 14.09,15.05$, $22.68,25.10,26.51,32.15,32.74,34.04,37.11,37.22,40.84,53.35,64.19,114.84,115.90$, 141.70, 151.20. MS (EI): $289\left(\mathrm{M}^{+}, 100\right), 290\left[(\mathrm{M}+\mathrm{H})^{+}, 20\right], 137$ (26), 108 (25), 176 (24), 218 (22), 246 (20), 163 (13); HRMS (ESI): Exact mass calcd for $\mathrm{C}_{19} \mathrm{H}_{31} \mathrm{O}_{1} \mathrm{~N}_{1} \mathrm{Na}[\mathrm{M}+\mathrm{Na}]^{+}$: 312.2298, Found: 312.2297

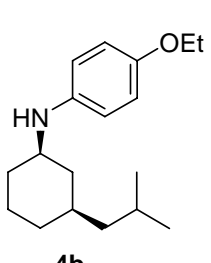

Column chromatography (5\% EtOAc in hexane) afforded the desired product 4b in $88 \%$ yield as colorless oil. HPLC analysis (Chiralcel AD-H, 2\% ${ }^{\mathrm{i}} \mathrm{PrOH} /$ heptane, $1.0 \mathrm{~mL} / \mathrm{min}, 247 \mathrm{~nm} ; \mathrm{t}_{\mathrm{r}}($ minor $)=7.83 \mathrm{~min}, \mathrm{t}_{\mathrm{r}}($ major $)=8.43$ $\min$ ) gave the isomeric composition of the product: er $=97.5: 2.5$. ${ }^{1} \mathrm{H}$ NMR $\left(400 \mathrm{MHz}, \mathrm{CDCl}_{3}\right): \delta 0.67(\mathrm{q}, J=11.8 \mathrm{~Hz}, 1 \mathrm{H}), 0.74-0.84(\mathrm{~m}, 1 \mathrm{H}), 0.85(\mathrm{~d}, J=$ $2.0 \mathrm{~Hz}, 3 \mathrm{H}), 0.86(\mathrm{~d}, J=2.0 \mathrm{~Hz}, 3 \mathrm{H}), 0.91-1.01(\mathrm{~m}, 1 \mathrm{H}), 1.03-1.13(\mathrm{~m}, 2 \mathrm{H}), 1.28-1.38(\mathrm{~m}$, $1 \mathrm{H}), 1.36(\mathrm{t}, J=7.0 \mathrm{~Hz}, 3 \mathrm{H}), 1.40-1.48(\mathrm{~m}, 1 \mathrm{H}), 1.61-1.81(\mathrm{~m}, 3 \mathrm{H}), 2.07-2.11(\mathrm{~m}, 2 \mathrm{H}), 2.5-$ 3.0 (br, 1H), 3.14 (tt, $J=11.2,3.6 \mathrm{~Hz}, 1 \mathrm{H}), 3.95$ (q, $J=6.8 \mathrm{~Hz}, 2 \mathrm{H}), 6.53-6.57$ (m, 2H), 6.73$6.78(\mathrm{~m}, 2 \mathrm{H}) .{ }^{13} \mathrm{C} \mathrm{NMR}\left(100 \mathrm{MHz}, \mathrm{CDCl}_{3}\right): \delta 15.04,22.80,22.89,24.74,25.07,32.93,34.01$, 34.63, 41.00, 46.76, 53.29, 64.15, 114.82, 115.85, 141.63, 151.17. MS (EI): 275 ( $\left.\mathrm{M}^{+}, 100\right)$, $276\left[(\mathrm{M}+\mathrm{H})^{+}, 20\right], 232$ (18), 108 (16), 218 (17), 163 (11), 137 (12), 246 (12); HRMS (ESI): Exact mass calcd for $\mathrm{C}_{18} \mathrm{H}_{29} \mathrm{O}_{1} \mathrm{~N}_{1} \mathrm{Na},[\mathrm{M}+\mathrm{Na}]^{+}:$298.2141, Found: 298.2143.

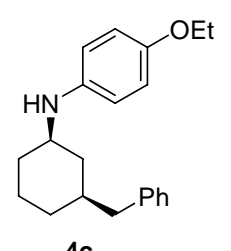

Column chromatography ( $3 \%$ EtOAc in hexane) afforded the desired product 4c in 77\% yield as colorless oil. HPLC analysis (Chiralcel OD-H, 5\% ${ }^{\mathrm{i}} \mathrm{PrOH} /$ heptane, $1.0 \mathrm{~mL} / \mathrm{min}, 247 \mathrm{~nm} ; \mathrm{t}_{\mathrm{r}}($ major $)=10.45 \mathrm{~min}, \mathrm{t}_{\mathrm{r}}($ minor $)=$ $14.59 \mathrm{~min}$ ) gave the isomeric composition of the product: er $=92.9: 7.1 .{ }^{1} \mathrm{H}$ NMR (400 MHz, $\left.\mathrm{CDCl}_{3}\right): \delta 0.81(\mathrm{q}, J=11.6 \mathrm{~Hz}, 1 \mathrm{H}), 0.81-1.01(\mathrm{~m}, 2 \mathrm{H})$, 1.23-1.32 (m, 1H), $1.36(\mathrm{t}, J=7.0 \mathrm{~Hz}, 3 \mathrm{H}), 1.62-1.79(\mathrm{~m}, 3 \mathrm{H}), 2.06-2.14$ (m, 2H), 2.53 (AB-d, $J=13.6,6.8 \mathrm{~Hz}, 2 \mathrm{H}), 2.5-3.0(\mathrm{br}, 1 \mathrm{H}), 3.11(\mathrm{tt}, J=11.2,3.6 \mathrm{~Hz}, 1 \mathrm{H}), 3.94(\mathrm{q}, J=7.0 \mathrm{~Hz}, 2 \mathrm{H})$, 
6.50-6.55 (m, 2H), 6.72-6.76 (m, 2H), 7.12-7.15 (m, 2H), 7.16-7.19 (m, 1H), 7.24-7.28 (m, $2 \mathrm{H}) ;{ }^{13} \mathrm{C} \mathrm{NMR}\left(100 \mathrm{MHz}, \mathrm{CDCl}_{3}\right): \delta 15.03,24.84,32.29,33.82,39.09,40.57,43.76,53.27$, 64.13, 114.80, 115.82, 125.78, 128, 14, 129.14, 140.66, 141.53, 151.20. MS (EI): $309\left(\mathrm{M}^{+}\right.$, 100), $310\left[(\mathrm{M}+\mathrm{H})^{+}, 23\right], 218$ (51), 108 (24), 176 (20), 163 (14), 137 (13), 280 (10); HRMS (ESI): Exact mass calcd for $\mathrm{C}_{21} \mathrm{H}_{27} \mathrm{O}_{1} \mathrm{~N}_{1} \mathrm{Na}[\mathrm{M}+\mathrm{Na}]^{+}$: 332.1985 , Found: 332.1987 .

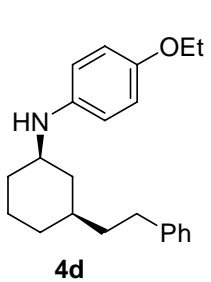

Column chromatography (3\% EtOAc in hexane) afforded the desired product 4d in 82\% yield as colorless oil. HPLC analysis (Chiralcel OD-H, 4\% ${ }^{\mathrm{i}} \mathrm{PrOH} /$ heptane, $1.0 \mathrm{~mL} / \mathrm{min}, 247 \mathrm{~nm}$; $\mathrm{t}_{\mathrm{r}}($ minor $)=12.33 \mathrm{~min}, \mathrm{t}_{\mathrm{r}}$ (major) $=21.42$ $\min )$ gave the isomeric composition of the product: er $=98 \cdot 0: 2.0$. ${ }^{1} \mathrm{H}$ NMR (400 MHz, $\left.\mathrm{CDCl}_{3}\right): \delta 0.76(\mathrm{q}, J=11.8 \mathrm{~Hz}, 1 \mathrm{H}), 0.84-1.03(\mathrm{~m}, 2 \mathrm{H}), 1.25-1.46(\mathrm{~m}, 5 \mathrm{H}), 1.48-$ $1.62(\mathrm{~m}, 2 \mathrm{H}), 1.78-1.83(\mathrm{~m}, 2 \mathrm{H}), 2.06-2.14(\mathrm{~m}, 1 \mathrm{H}), 2.16-2.24(\mathrm{~m}, 1 \mathrm{H}), 2.63(\mathrm{t}, J=8.0 \mathrm{~Hz}$, 2H), 2.5-3.0 (br, 1H), 3.13 (tt, $J=11.2,3.6 \mathrm{~Hz}, 1 \mathrm{H}), 3.95$ (q, $J=7.0 \mathrm{~Hz}, 2 \mathrm{H}), 6.53-6.57$ (m, $2 \mathrm{H}), 6.74-6.78(\mathrm{~m}, 2 \mathrm{H}), 7.15-7.19(\mathrm{~m}, 3 \mathrm{H}), 7.25-7.29(\mathrm{~m}, 2 \mathrm{H}) ;{ }^{13} \mathrm{C} \mathrm{NMR}\left(100 \mathrm{MHz}, \mathrm{CDCl}_{3}\right)$ : $\delta 15.04,24.97,32.63,33.15,33.92,36.57,39.00,40.53,53.18,64.14,114.84,115.85,125.63$, 128.30, 141.54, 142.78, 151.21. MS (EI): $323\left(\mathrm{M}^{+}, 100\right), 324\left[(\mathrm{M}+\mathrm{H})^{+}, 24\right], 108$ (21), 176 (20), 218 (19), 280 (13), 137 (13), 294 (9); HRMS (ESI): Exact mass calcd for $\mathrm{C}_{22} \mathrm{H}_{29} \mathrm{O}_{1} \mathrm{~N}_{1} \mathrm{Na}$ $[\mathrm{M}+\mathrm{Na}]^{+}:$346.2141, Found: 346.2141.

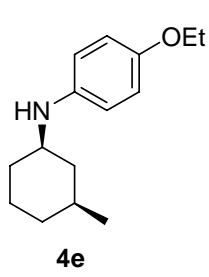

Column chromatography (5\% EtOAc in hexane) afforded the desired product 4e in $88 \%$ yield as colorless oil. HPLC analysis (Chiralcel OB-H, 4\% ${ }^{\mathrm{i}} \mathrm{PrOH} /$ heptane, $1.0 \mathrm{~mL} / \mathrm{min}, 247 \mathrm{~nm} ; \mathrm{t}_{\mathrm{r}}($ minor $)=8.55 \mathrm{~min}, \mathrm{t}_{\mathrm{r}}$ (major) $=10.74$ $\min )$ gave the isomeric composition of the product: er $=91.9: 8.1 .{ }^{1} \mathrm{H}$ NMR (400 MHz, $\left.\mathrm{CDCl}_{3}\right): \delta 0.71(\mathrm{q}, J=12.0 \mathrm{~Hz}, 1 \mathrm{H}), 0.73-0.95(\mathrm{~m}, 2 \mathrm{H}), 0.88(\mathrm{~d}, J=6.8 \mathrm{~Hz}, 3 \mathrm{H})$, $1.29-1.41(\mathrm{~m}, 1 \mathrm{H}), 1.36(\mathrm{t}, J=6.8 \mathrm{~Hz}, 3 \mathrm{H}), 1.43-1.54(\mathrm{~m}, 1 \mathrm{H}), 1.64-1.72(\mathrm{~m}, 1 \mathrm{H}), 1.73-1.81$ (m, 1H), 2.06-2.11 (m, 2H), 2.5-3.0 (br, 1H), 3.14 (tt, $J=11.2,3.6 \mathrm{~Hz}, 1 \mathrm{H}), 3.95$ (q, $J=6.8$ $\mathrm{Hz}, 2 \mathrm{H}), 6.54-6.57$ (m, 2H), 6.74-6.78 (m, 2H). $\left.{ }^{13} \mathrm{C} \mathrm{NMR} \mathrm{(100} \mathrm{MHz,} \mathrm{CDCl}_{3}\right): \delta 15.04,22.54$, 25.10, 32.09, 33.57, 34.73, 42.70, 53.28, 64.16, 114.91, 115.84, 141.62, 151.21. MS (EI): 233 $\left(\mathrm{M}^{+}, 100\right), 234\left[(\mathrm{M}+\mathrm{H})^{+}, 20\right], 190$ (48), 108 (32), 176 (30), 204 (24), 163 (13), 218 (8); HRMS (ESI): Exact mass calcd for $\mathrm{C}_{15} \mathrm{H}_{24} \mathrm{O}_{1} \mathrm{~N}_{1}[\mathrm{M}+\mathrm{H}]^{+}$: 234.1852, Found: 234.1852 .

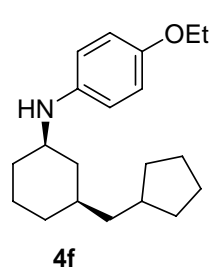

Column chromatography (4\% EtOAc in hexane) afforded the desired product 4f in $72 \%$ yield as colorless oil. HPLC analysis (Chiralcel AD-H, 4\% ${ }^{\mathrm{i}} \mathrm{PrOH} /$ heptane, $1.0 \mathrm{~mL} / \mathrm{min}, 247 \mathrm{~nm} ; \mathrm{t}_{\mathrm{r}}($ minor $)=6.44 \mathrm{~min}, \mathrm{t}_{\mathrm{r}}$ (major) $=7.01$ 
min) gave the isomeric composition of the product: er $=97.8: 2.2 .{ }^{1} \mathrm{H}$ NMR $(400 \mathrm{MHz}$, $\left.\mathrm{CDCl}_{3}\right): \delta 0.69(\mathrm{q}, J=11.6 \mathrm{~Hz}, 1 \mathrm{H}), 0.75-0.86(\mathrm{~m}, 1 \mathrm{H}), 0.90-1.08(\mathrm{~m}, 3 \mathrm{H}), 1.16-1.26(\mathrm{~m}, 2 \mathrm{H})$, 1.27-1.44 (m, 2H), $1.36(\mathrm{t}, J=7.0 \mathrm{~Hz}, 3 \mathrm{H}), 1.45-1.53(\mathrm{~m}, 2 \mathrm{H}), 1.54-1.64(\mathrm{~m}, 2 \mathrm{H}), 1.69-1.82$ $(\mathrm{m}, 4 \mathrm{H}), 1.82-1.90(\mathrm{~m}, 1 \mathrm{H}), 2.08-2.14(\mathrm{~m}, 2 \mathrm{H}), 2.5-3.0(\mathrm{br}, 1 \mathrm{H}), 3.13(\mathrm{tt}, J=11.2,3.6 \mathrm{~Hz}, 1 \mathrm{H})$, 3.95 (q, $J=7.0 \mathrm{~Hz}, 2 \mathrm{H}), 6.53-6.57(\mathrm{~m}, 2 \mathrm{H}), 6.74-6.78(\mathrm{~m}, 2 \mathrm{H}) .{ }^{13} \mathrm{C}$ NMR $\left(100 \mathrm{MHz}, \mathrm{CDCl}_{3}\right)$ : $\delta 15.04,25.08,25.12,32.95,32.99,33.01,34.00,36.14,37.20,41.06,43.92,53.31,64.14$, 114.82, 115.84, 141.65, 151.16. MS (EI): $301\left(\mathrm{M}^{+}, 100\right), 302\left[(\mathrm{M}+\mathrm{H})^{+}, 22\right], 176(21), 108$ (19), 218 (18), 258 (11), 137 (16), 163 (11); HRMS (ESI): Exact mass calcd for $\mathrm{C}_{15} \mathrm{H}_{24} \mathrm{O}_{1} \mathrm{~N}_{1}$ $\mathrm{Na}[\mathrm{M}+\mathrm{Na}]^{+}: 324.2298$, Found: 324.2297.

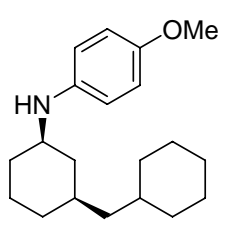

$4 \mathrm{~g}$

Column chromatography (3\% EtOAc in hexane) afforded the desired product $\mathbf{4 g}$ in $89 \%$ yield as colorless oil. HPLC analysis (Chiralcel AD-H, 4\% ${ }^{\mathrm{i}} \mathrm{PrOH} /$ heptane, $1.0 \mathrm{~mL} / \mathrm{min}, 247 \mathrm{~nm}$; $\mathrm{t}_{\mathrm{r}}$ (minor) $=10.18 \mathrm{~min}, \mathrm{t}_{\mathrm{r}}$ (major) $=11.17$ $\min )$ gave the isomeric composition of the product: er $=98.0: 2.0$. ${ }^{1} \mathrm{H}$ NMR $\left(400 \mathrm{MHz}, \mathrm{CDCl}_{3}\right): \delta 0.67(\mathrm{q}, J=11.6 \mathrm{~Hz}, 1 \mathrm{H}), 0.77-0.89(\mathrm{~m}, 3 \mathrm{H}), 0.89-1.02(\mathrm{~m}, 1 \mathrm{H}), 1.05-$ $1.11(\mathrm{~m}, 2 \mathrm{H}), 1.14-1.25(\mathrm{~m}, 3 \mathrm{H}), 1.26-1.38(\mathrm{~m}, 2 \mathrm{H}), 1.42-1.54(\mathrm{~m}, 1 \mathrm{H}), 1.60-1.78(\mathrm{~m}, 6 \mathrm{H})$, 1.75-1.82 (m, 1H), 2.06-2.14 (m, 2H), 2.5-3.0 (br, 1H), 3.13 (tt, $J=11.2,3.6 \mathrm{~Hz}, 1 \mathrm{H}), 3.74$ (s, $3 \mathrm{H}), 6.54-6.58(\mathrm{~m}, 2 \mathrm{H}), 6.74-6.78(\mathrm{~m}, 2 \mathrm{H}) .{ }^{13} \mathrm{C} \mathrm{NMR}\left(100 \mathrm{MHz}, \mathrm{CDCl}_{3}\right): \delta 25.06,26.41$, $26.74,33.03,33.65,33.74,33.85,33.98,34.40,41.10,45.23,53.30,55.84,114.85,114.95$, 141.64, 151.90. MS (EI): $301\left(\mathrm{M}^{+}, 100\right), 302\left[(\mathrm{M}+\mathrm{H})^{+}, 22\right], 162$ (29), 123 (26), 204 (20), 149 (16), 136 (13), 108 (10); HRMS (ESI): Exact mass calcd for $\mathrm{C}_{20} \mathrm{H}_{31} \mathrm{O}_{1} \mathrm{~N}_{1} \mathrm{Na}[\mathrm{M}+\mathrm{Na}]^{+}$: 324.2298, Found: 324.2297.

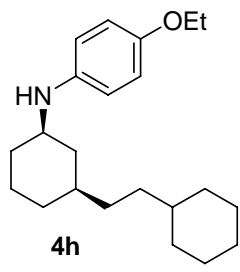

Column chromatography (3\% EtOAc in hexane) afforded the desired product 4h in 78\% yield as colorless oil. HPLC analysis (Chiralcel AD-H, 5\% ${ }^{\mathrm{i}} \mathrm{PrOH} /$ heptane, $1.0 \mathrm{~mL} / \mathrm{min}, 247 \mathrm{~nm} ; \mathrm{t}_{\mathrm{r}}$ (major) $=6.95 \mathrm{~min}, \mathrm{t}_{\mathrm{r}}$ (minor) $=8.76$ min) gave the isomeric composition of the product: er $=96.4: 3.6 .{ }^{1} \mathrm{H}$ NMR $\left(400 \mathrm{MHz}, \mathrm{CDCl}_{3}\right): \delta 0.69(\mathrm{q}, J=11.5 \mathrm{~Hz}, 1 \mathrm{H}), 0.76-0.91(\mathrm{~m}, 3 \mathrm{H}), 0.90-1.00$ (m, 1H), 1.11-1.24 (m, 8H), 1.25-1.35 (m, 2H), $1.36(\mathrm{t}, J=6.8 \mathrm{~Hz}, 3 \mathrm{H}), 1.61-1.80(\mathrm{~m}, 7 \mathrm{H})$, 2.07-2.12 (m, 2H), 2.5-3.0 (br, 1H), 3.12 (tt, $J=11.2,3.6 \mathrm{~Hz}, 1 \mathrm{H}), 3.95$ (q, $J=7.0 \mathrm{~Hz}, 2 \mathrm{H})$, 6.52-6.57 (m, 2H), 6.74-6.78 (m, 2H). ${ }^{13} \mathrm{C}$ NMR (100 MHz, $\left.\mathrm{CDCl}_{3}\right): \delta 15.05,25.10,26.46$, 26.78, 32.78, 33.48, 34.04, 34.47, 34.65, 37.42, 37.96, 40.86, 53.36, 64.19, 114.84, 115.89, 141.70, 151.19. MS (EI): $329\left(\mathrm{M}^{+}, 100\right), 330\left[(\mathrm{M}+\mathrm{H})^{+}, 22\right], 137$ (21), 218 (16), 176 (15), 108 (14), 163 (9), 286 (8); HRMS (ESI): Exact mass calcd for $\mathrm{C}_{22} \mathrm{H}_{36} \mathrm{O}_{1} \mathrm{~N}_{1}[\mathrm{M}+\mathrm{H}]^{+}: 330.2791$, 
Found: 330.2794.

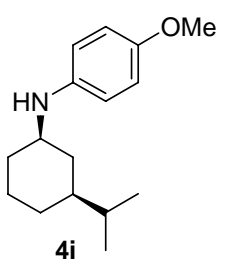

Column chromatography (4\% EtOAc in hexane) afforded the desired product $4 \mathbf{i}$ in $76 \%$ yield as colorless oil. HPLC analysis (Chiralcel OJ-H, 2\% ${ }^{\mathrm{i}} \mathrm{PrOH} / \mathrm{heptane}, 1.0 \mathrm{~mL} / \mathrm{min}, 247 \mathrm{~nm}$; $\mathrm{t}_{\mathrm{r}}$ (major) $=13.63 \mathrm{~min}, \mathrm{t}_{\mathrm{r}}($ minor $)=17.61$ min) gave the isomeric composition of the product: er $=96.4: 3.6$. ${ }^{1} \mathrm{H}$ NMR $\left(300 \mathrm{MHz}, \mathrm{CDCl}_{3}\right): \delta 0.78(\mathrm{q}, J=12.0 \mathrm{~Hz}, 1 \mathrm{H}), 0.86(\mathrm{~d}, J=6.8 \mathrm{~Hz}, 6 \mathrm{H}), 0.86-1.12(\mathrm{~m}, 2 \mathrm{H})$, 1.15-1.40 (m, 2H), 1.40-1.52 (m, 1H), 1.64-1.74 (m, 1H), 1.76-1.88 (m, 1H), 2.07-2.11 (m, 2H), 2.5-3.0 (br, 1H), 3.13 (tt, $J=11.2,3.6 \mathrm{~Hz}, 1 \mathrm{H}), 3.74$ (s, 3H), 6.53-6.59 (m, 2H), 6.73$6.78(\mathrm{~m}, 2 \mathrm{H}) .{ }^{13} \mathrm{C}$ NMR $\left(75 \mathrm{MHz}, \mathrm{CDCl}_{3}\right): \delta$ 19.66, 19.72, 25.15, 29.08, 32.74, 34.02, 37.35, 43.33, 53.64, 55.85, 114.84, 114.96, 141.65, 151.90. MS (EI): $247\left(\mathrm{M}^{+}, 100\right), 248\left[(\mathrm{M}+\mathrm{H})^{+}\right.$, 22], 204 (54), 162 (44), 123 (28), 149 (22), 108 (17), 136 (12); HRMS (ESI): Exact mass calcd for $\mathrm{C}_{16} \mathrm{H}_{26} \mathrm{O}_{1} \mathrm{~N}_{1}[\mathrm{M}+\mathrm{H}]^{+}$: 248.2009, Found: 248.2008.

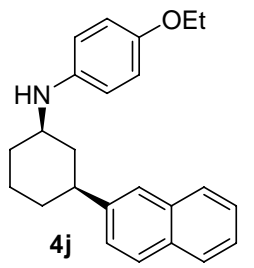

Column chromatography $\left(\mathrm{CH}_{2} \mathrm{Cl}_{2}\right)$ afforded the desired product $\mathbf{4 j}$ in $\mathbf{7 3 \%}$ yield as colorless oil. HPLC analysis (Chiralcel AD-H, 5\% ${ }^{\mathrm{i}} \mathrm{PrOH} /$ heptane, $1.0 \mathrm{~mL} / \mathrm{min}, 247 \mathrm{~nm} ; \mathrm{t}_{\mathrm{r}}($ minor $)=19.56 \mathrm{~min}, \mathrm{t}_{\mathrm{r}}($ major $\left.)=21.10 \mathrm{~min}\right)$ gave the isomeric composition of the product: er $=91.2: 8.8 .{ }^{1} \mathrm{H}$ NMR $(400 \mathrm{MHz}$, $\left.\mathrm{CDCl}_{3}\right): \delta 1.11-1.22(\mathrm{~m}, 1 \mathrm{H}), 1.35(\mathrm{t}, J=7.0 \mathrm{~Hz}, 3 \mathrm{H}), 1.32-1.41(\mathrm{~m}, 1 \mathrm{H}), 1.43-1.63(\mathrm{~m}, 2 \mathrm{H})$, 1.95-2.05 (m, 2H), 2.18-2.26 (m, 1H), 2.38-2.44 (m, 1H), 2.8-3.2 (br, 1H), $2.82(\mathrm{tt}, J=12.0$, $3.2 \mathrm{~Hz}, 1 \mathrm{H}), 3.37$ (tt, $J=11.2,3.6 \mathrm{~Hz}, 1 \mathrm{H}), 3.94$ (q, $J=7.0 \mathrm{~Hz}, 2 \mathrm{H}), 6.58-6.62$ (m, 2H), 6.74$6.78(\mathrm{~m}, 2 \mathrm{H}), 7.35-7.45(\mathrm{~m}, 3 \mathrm{H}), 7.62(\mathrm{t}, J=0.7 \mathrm{~Hz}, 1 \mathrm{H}), 7.75-7.80(\mathrm{~m}, 3 \mathrm{H}) .{ }^{13} \mathrm{C}$ NMR $(100$ $\left.\mathrm{MHz}, \mathrm{CDCl}_{3}\right): \delta 15.02,25.47,33.63,33.92,41.20,43.51,53.69,64.14,115.05,115.86$, $124.59,125.20,125.89,125.95,127.55,127.58,127.89,132.20,133.63,141.33,143.95$, 151.37. MS (EI): $345\left(\mathrm{M}^{+}, 100\right), 346\left[(\mathrm{M}+\mathrm{H})^{+}, 27\right], 141$ (36), 137 (34), 302 (30), 176 (14), 163 (13), 108 (8); HRMS (ESI): Exact mass calcd for $\mathrm{C}_{24} \mathrm{H}_{27} \mathrm{O}_{1} \mathrm{~N}_{1} \mathrm{Na}[\mathrm{M}+\mathrm{Na}]^{+}: 368.1985$, Found: 368.1985.

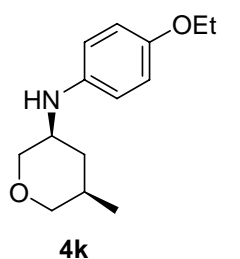

Column chromatography (hexane/EtOAc: 10/1) afforded the desired product 4k in 72\% yield as yellow solid. HPLC analysis (Chiralcel AD-H, 5\% ${ }^{\mathrm{i}} \mathrm{PrOH} /$ heptane, $1.0 \mathrm{~mL} / \mathrm{min}, 247 \mathrm{~nm} ; \mathrm{t}_{\mathrm{r}}($ major $)=15.61 \mathrm{~min}, \mathrm{t}_{\mathrm{r}}($ minor $)=17.87$ min) gave the isomeric composition of the product: er $=96.4: 3.6$. ${ }^{1} \mathrm{H}$ NMR $\left(400 \mathrm{MHz}, \mathrm{CDCl}_{3}\right): \delta 0.85(\mathrm{~d}, J=6.6 \mathrm{~Hz}, 3 \mathrm{H}), 0.92(\mathrm{q}, J=12 \mathrm{~Hz}, 1 \mathrm{H}), 1.36(\mathrm{t}, J=6.8 \mathrm{~Hz}$, $3 \mathrm{H}), 1.83-1.90(\mathrm{~m}, 1 \mathrm{H}), 2.15-2.21(\mathrm{~m}, 1 \mathrm{H}), 2.87$ (m, 2H), 3.0-3.3 (br, 1H), 3.37-3.45 (m, 1H), 
$3.85(\mathrm{ddd}, J=13.2,4.4,1.8 \mathrm{~Hz}, 1 \mathrm{H}), 3.95$ (q, $J=6.8 \mathrm{~Hz}, 2 \mathrm{H}), 4.15(\mathrm{ddd}, J=13.2,4.4,2.0 \mathrm{~Hz}$, $1 \mathrm{H}), 6.58-6.60(\mathrm{~m}, 2 \mathrm{H}), 6.74-6.78(\mathrm{~m}, 2 \mathrm{H}) .{ }^{13} \mathrm{C} \mathrm{NMR}\left(100 \mathrm{MHz}, \mathrm{CDCl}_{3}\right): \delta 15.01,16.94$, 31.05, 40.10, 50.36, 64.18, 72.03, 74.28, 114.74, 115.96, 140.84, 151.68. MS (EI): $235\left(\mathrm{M}^{+}\right.$, 100), $236\left[(\mathrm{M}+\mathrm{H})^{+}, 15\right], 190$ (87), 163 (38), 134 (26), 206 (13), 178 (11), 108 (10); HRMS (EI): Exact mass calcd for $\mathrm{C}_{14} \mathrm{H}_{21} \mathrm{~N}_{1} \mathrm{O}_{2}[\mathrm{M}]^{+}$: 235.1572, Found: 235.1570 .

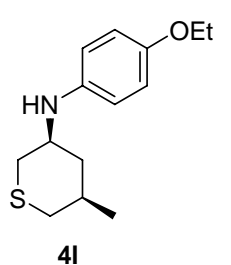

Column chromatography (first using $\mathrm{CH}_{2} \mathrm{Cl}_{2}$ to separate trans/cis product from oxidized Hantzsch ester, then using hexane/acetate 25/1 to separate trans and cis product) afforded the desired product $\mathbf{4 l}$ in $35 \%$ yield as yellow oil. HPLC analysis (Chiralcel AD-H, 5\% ${ }^{\mathrm{i}} \mathrm{PrOH} /$ heptane, $1.0 \mathrm{~mL} / \mathrm{min}, 247 \mathrm{~nm}$; $\mathrm{t}_{\mathrm{r}}$ (major) $=16.89 \mathrm{~min}, \mathrm{t}_{\mathrm{r}}($ minor $\left.)=21.42 \mathrm{~min}\right)$ gave the isomeric composition of the product: er $=$ 95.0:5.0. ${ }^{1} \mathrm{H}$ NMR (400 MHz, $\left.\mathrm{CDCl}_{3}\right): \delta^{1} \mathrm{H} \mathrm{NMR}\left(400 \mathrm{MHz}, \mathrm{CDCl}_{3}\right): \delta 0.88(\mathrm{q}, J=12.4 \mathrm{~Hz}$, $1 \mathrm{H}), 0.99(\mathrm{~d}, J=6.4 \mathrm{~Hz}, 3 \mathrm{H}), 1.36(\mathrm{t}, J=6.8 \mathrm{~Hz}, 3 \mathrm{H}), 1.92-2.02(\mathrm{~m}, 1 \mathrm{H}), 2.06-2.10(\mathrm{~m}, 1 \mathrm{H})$, 2.16-2.24 (m, 2H), 2.44-2.49 (m, 1H), 2.87-2.92 (m, 1H), 3.0-3.4 (br, 1H), 3.47 (tt, $J=10.0$, $3.4 \mathrm{~Hz}, 1 \mathrm{H}), 3.95$ (q, $J=6.8 \mathrm{~Hz}, 2 \mathrm{H}), 6.55-6.60(\mathrm{~m}, 2 \mathrm{H}), 6.75-6.79(\mathrm{~m}, 2 \mathrm{H}) .{ }^{13} \mathrm{C}$ NMR (100 $\left.\mathrm{MHz}, \mathrm{CDCl}_{3}\right): \delta 15.01,22.68,33.56,35.24,35.25,42.58,53.72,64.15,115.09,115.98$, 140.36, 151.73. MS (EI): $251\left(\mathrm{M}^{+}, 70\right), 252\left[(\mathrm{M}+\mathrm{H})^{+}, 11\right], 115$ (100), 190 (40), 163 (38), 134 (22), 150 (21), 81 (15), 108 (10); HRMS (EI): Exact mass calcd for $\mathrm{C}_{14} \mathrm{H}_{21} \mathrm{~N}_{1} \mathrm{O}_{1} \mathrm{~S}_{1}[\mathrm{M}]^{+}$: 251.1341, Found: 251.1344.

\section{Deprotection and Determination of the Absolute Configuration}

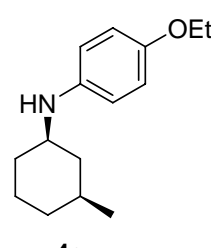

$4 \mathrm{e}$

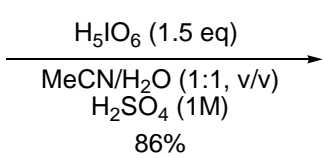

$86 \%$

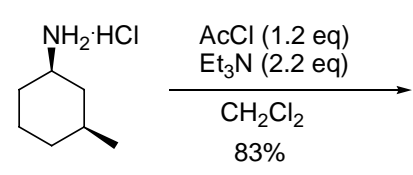

$13 e$

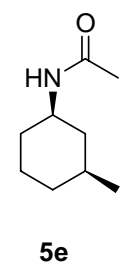

$5 e$

To a solution of cis-N-(p-ethoxyphenyl)-3-methylcyclohexaneamine (233 $\mathrm{mg}, 83 \%$ ee, 1 $\mathrm{mmol})$ in $\mathrm{MeCN} / \mathrm{H}_{2} \mathrm{O}(20 \mathrm{~mL}, 1: 1, \mathrm{v} / \mathrm{v})$ was added $\mathrm{H}_{5} \mathrm{IO}_{6}(375 \mathrm{mg}, 1.5 \mathrm{mmol})$ and $1 \mathrm{M}$ aqueous solution of $\mathrm{H}_{2} \mathrm{SO}_{4}(1 \mathrm{~mL}) .{ }^{9}$ The mixture was stirred for 14 hours at room temperature, and then washed with $\mathrm{CH}_{2} \mathrm{Cl}_{2}(4 \times 75 \mathrm{~mL})$. The organic phase was discarded and $5 \mathrm{M}$ aqueous $\mathrm{KOH}$ solution was added drop wise to the resulting colorless aqueous phase until $\mathrm{pH}$

(9) Verkade, J. M. M.; van Hermert, L. J. C.; Quaedflieg, P. J. L. M.; Alsters, P. L.; van Delft, F. L.; Rutjes, F. P. J. T. Tetrahedron Lett. 2006, 47, 8109-8113 
11 was reached. The basic aqueous solution was extracted with EtOAc $(4 \times 100 \mathrm{~mL})$. The combined organic layer were brought to $\mathrm{pH} 1$ via addition of ${ }^{i} \mathrm{PrOH} / \mathrm{HCl}$, dried over $\mathrm{MgSO}_{4}$, and concentrated to afford the $\mathrm{HCl}$ salt $13 \mathrm{e}\left(128 \mathrm{mg}, 86 \%\right.$ yield) as slightly yellow solid. ${ }^{1} \mathrm{H}$ $\operatorname{NMR}\left(400 \mathrm{MHz}, \mathrm{D}_{2} \mathrm{O}\right): \delta 0.82(\mathrm{qd}, J=12.6,3.6 \mathrm{~Hz}, 1 \mathrm{H}), 0.91(\mathrm{~d}, J=6.4 \mathrm{~Hz}, 3 \mathrm{H}), 0.98(\mathrm{q}, J$ $=12.0 \mathrm{~Hz}, 1 \mathrm{H}), 1.14-1.37(\mathrm{~m}, 2 \mathrm{H}), 1.41-1.51(\mathrm{~m}, 1 \mathrm{H}), 1.63-1.66(\mathrm{~m}, 1 \mathrm{H}), 1.77-1.82(\mathrm{~m}, 1 \mathrm{H})$, 1.90-1.98 (m, 2H), 3.09-3.18 (m, 1H). ${ }^{13} \mathrm{C}$ NMR (100 MHz, $\left.\mathrm{D}_{2} \mathrm{O}\right): \delta 21.85,24.07,30.39$, $31.96,33.40,39.04,50.84$.

To a solution of the above prepared amine salt $(108 \mathrm{mg}, 0.73 \mathrm{mmol})$ in dichloromethane $(10 \mathrm{~mL})$ was added drop wise $\mathrm{Et}_{3} \mathrm{~N}(0.22 \mathrm{~mL}, 2.2 \mathrm{eq})$ and $\mathrm{AcCl}(60 \mu \mathrm{L}, 1.2 \mathrm{eq})$. The resulting mixture was stirred at room temperature for 6 hours, and then $20 \mathrm{~mL}$ of water was added. The mixture was extracted with $\mathrm{CH}_{2} \mathrm{Cl}_{2}(3 \times 30 \mathrm{~mL})$, and the organic phases were combined, dried and concentrated. The crude mixture was purified by column chromatography using gradient eluent (50\% EtOAc in hexane first, then 75\%) afforded the desired product (94 $\mathrm{mg}, 83 \%$ yield) as white solid. ${ }^{1} \mathrm{H}$ NMR $\left(400 \mathrm{MHz}, \mathrm{D}_{2} \mathrm{O}\right): \delta 0.71(\mathrm{q}, J=12.0 \mathrm{~Hz}, 1 \mathrm{H}), 0.78(\mathrm{qd}, J=$ 13.0, 3.6 Hz, 1H), $0.90(\mathrm{~d}, J=6.4 \mathrm{~Hz}, 3 \mathrm{H}), 0.95(\mathrm{qd}, J=12.5,3.8 \mathrm{~Hz}, 1 \mathrm{H}), 1.36$ (qt, $J=13.2$, $3.6 \mathrm{~Hz}, 1 \mathrm{H}), 1.46-1.56(\mathrm{~m}, 1 \mathrm{H}), 1.60-1.68(\mathrm{~m}, 2 \mathrm{H}), 1.70-1.78(\mathrm{~m}, 1 \mathrm{H}), 1.92-1.96(\mathrm{~m}, 1 \mathrm{H})$, $1.95(\mathrm{~s}, 3 \mathrm{H}), 3.75(\mathrm{~m}, 1 \mathrm{H}), 5.24(\mathrm{~s}, \mathrm{br}, 1 \mathrm{H}) .{ }^{13} \mathrm{C} \mathrm{NMR}\left(100 \mathrm{MHz}, \mathrm{CDCl}_{3}\right): \delta$ 22.40, 23.59, $24.82,31.79,32.99,34.27,42.10,48.59,169.11 .[\alpha]_{\mathrm{D}}{ }^{20}=+30.8(\mathrm{c}=0.60$, benzene $)$; reported rotation for $(1 S, 3 R)-N$-acetyl-3- methylcyclohexylamine $[\alpha]_{\mathrm{D}}{ }^{20}=-43.4(\mathrm{c}=0.31$, benzene $){ }^{10}$

(10) a) Demailly, G:; Solladie, G. J. Org. Chem. 1981, 46, 3102. b) Huckel, W.; Thomas, H. D. Justus Liebigs Ann. Chem. 1961, 645, 77. 

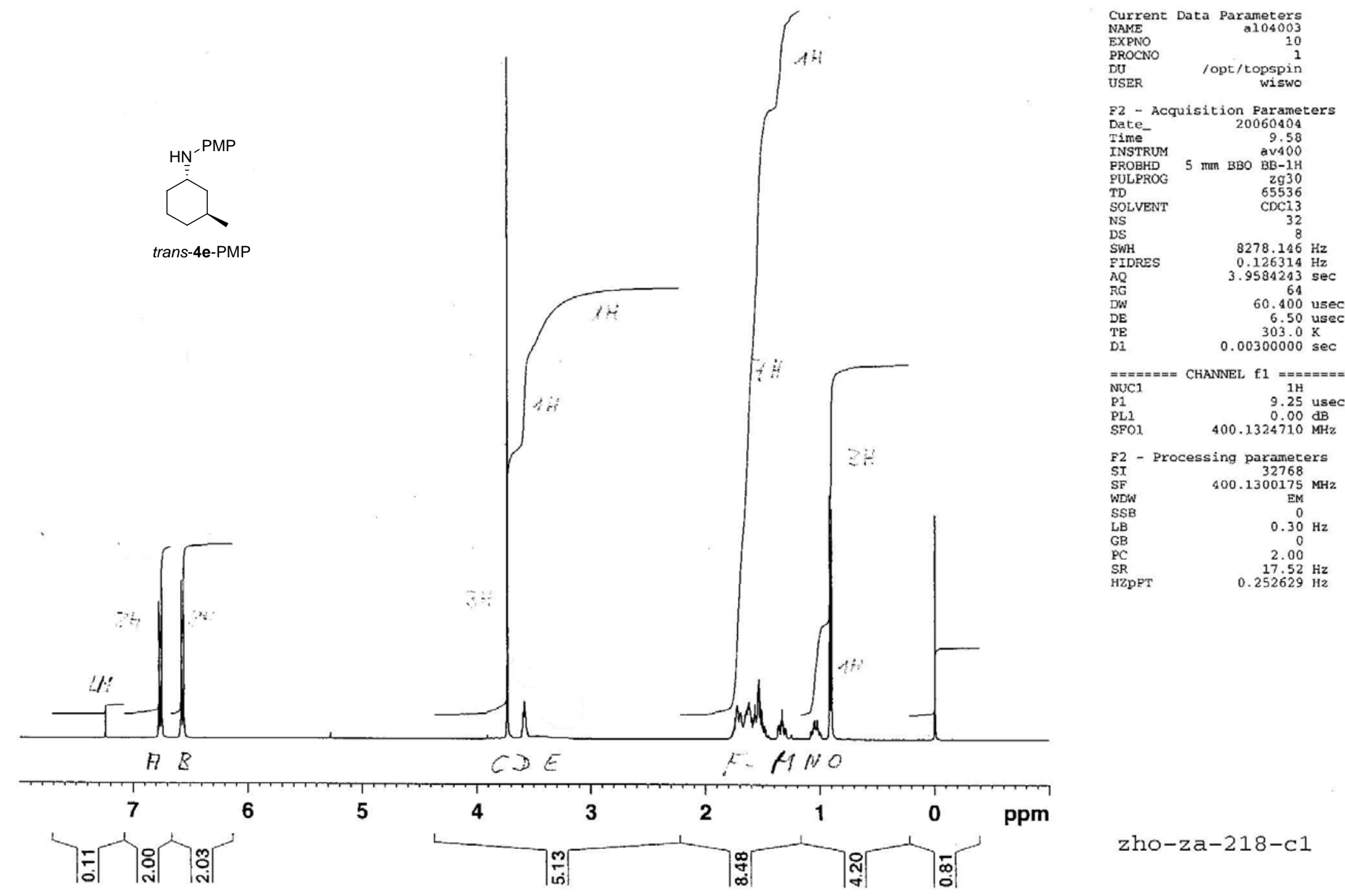
zho-za-218-c1

lopthopspir/wiswo/al04003/10/1

Integrai regions \#4 to $\# 4$
$20 \mathrm{~Hz} / \mathrm{cm}$

$20 \mathrm{~Hz} / \mathrm{Cr}$
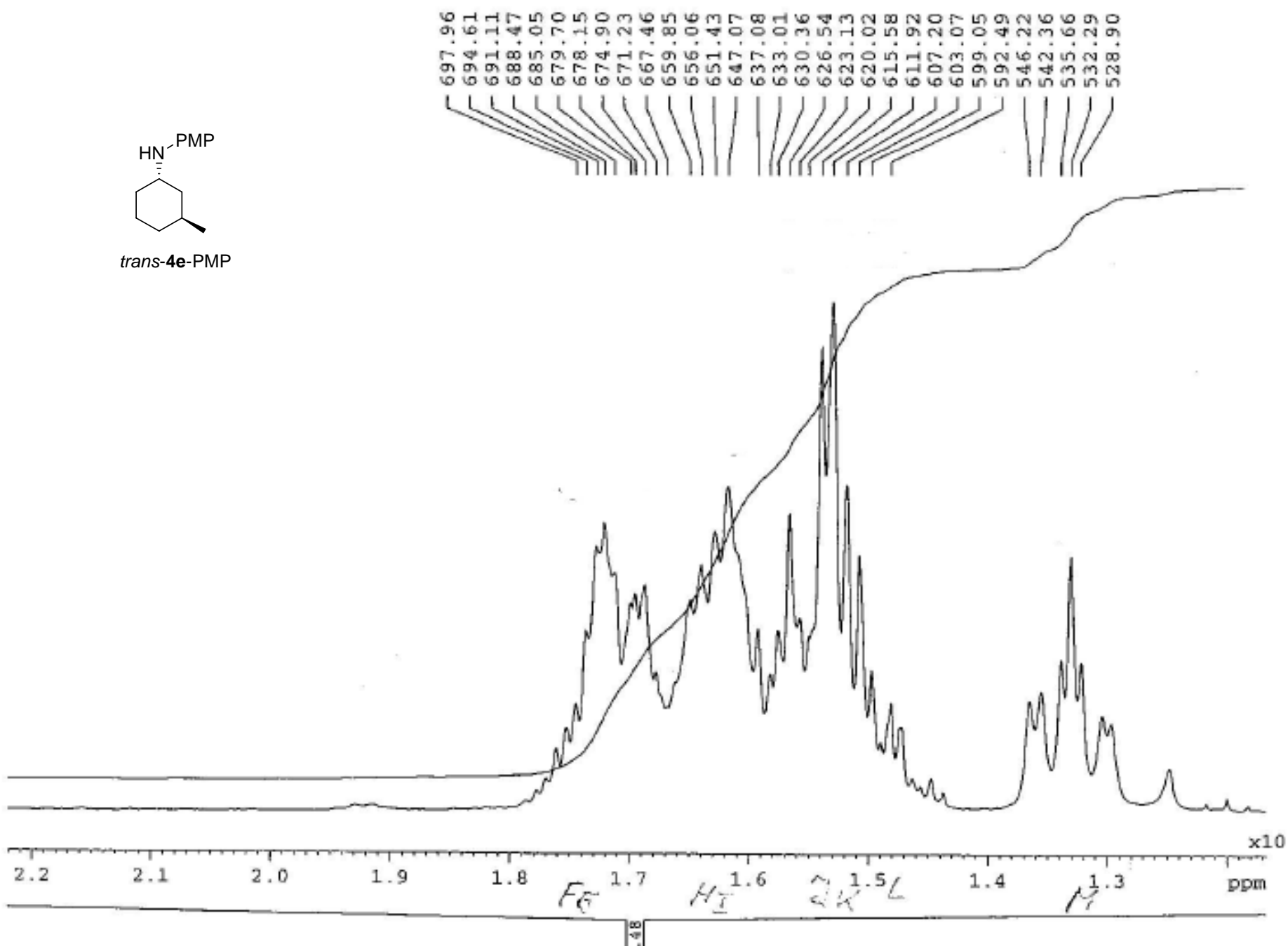

$\mid$

-S 17- 
mr31059 11 i $C:\lfloor$ Bruker \TOPSEIM tho

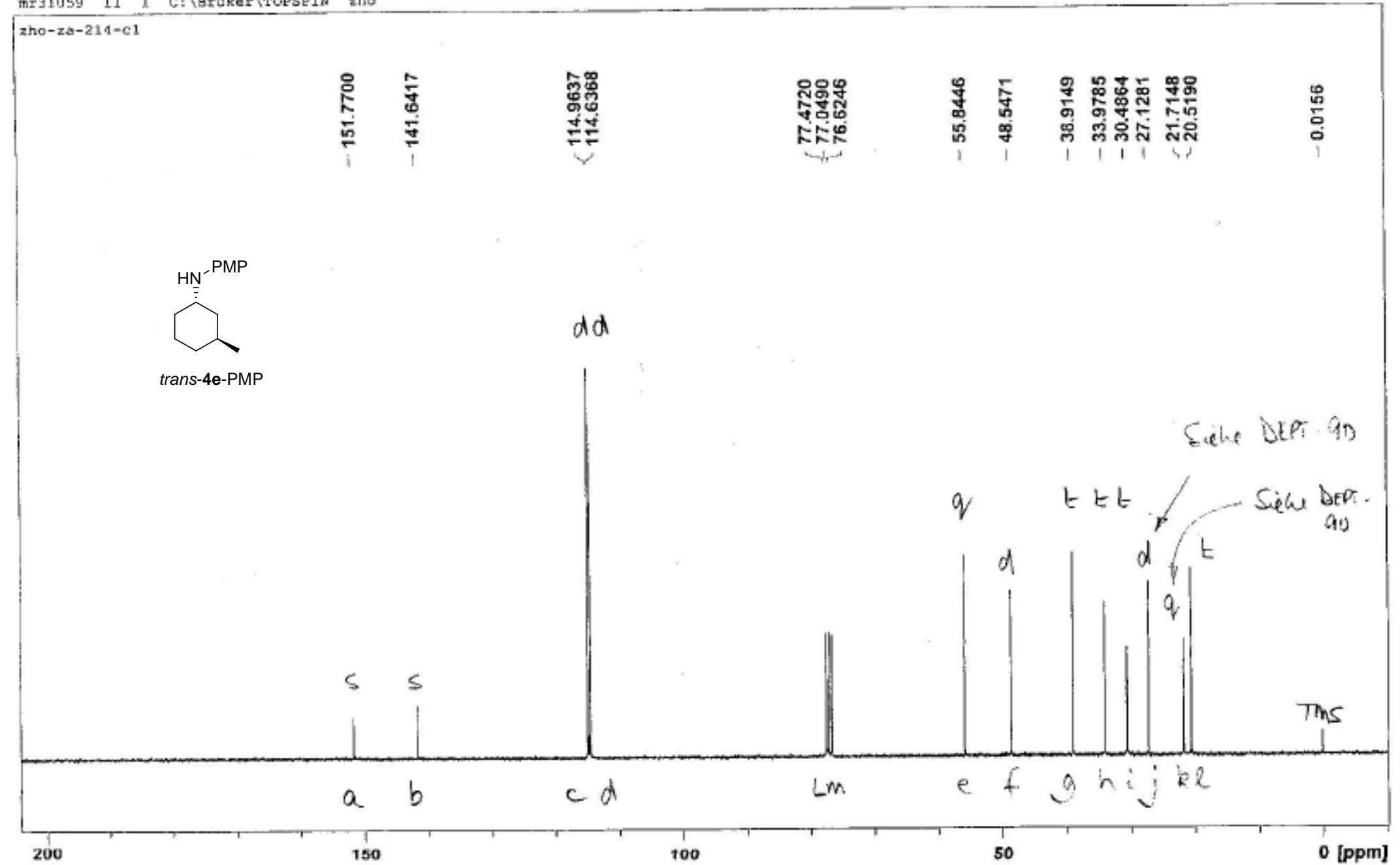


E

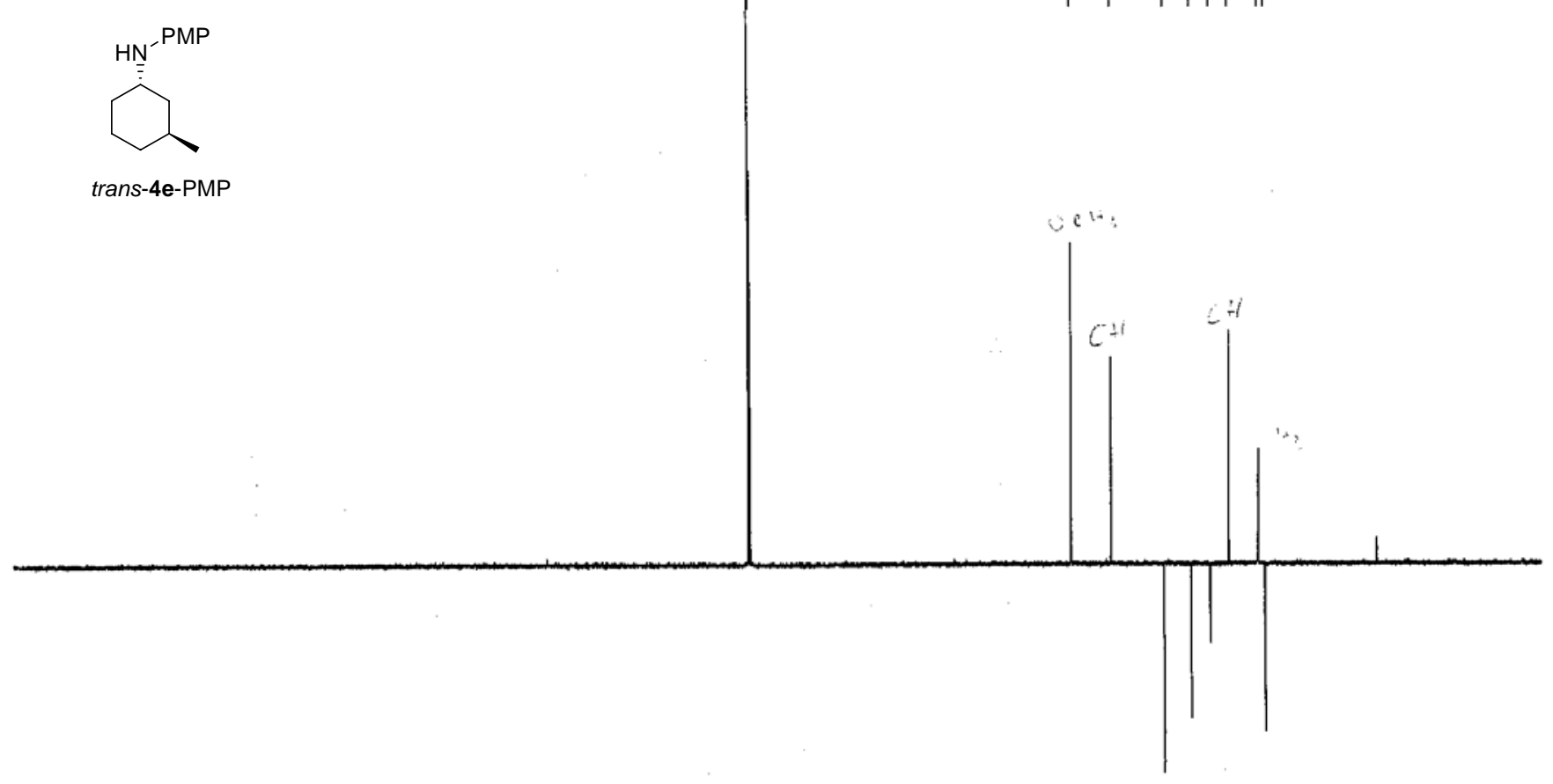

Current Dato Parsmeters
Naye al

NAME
EXPNO
PROCNO

F2 - Acquisstion Paraneters

$\begin{array}{ll}\text { Dote- } & 20060003 \\ \text { Time } & 11.20 \\ & \end{array}$

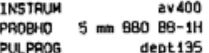

$\quad 65536$

$\begin{array}{lc}\mathrm{NS} & 320 \\ \mathrm{OS} & 4 \\ 5 \mathrm{NH} & 30120.482 \\ & \end{array}$

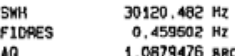

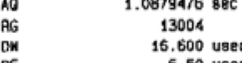

$\begin{array}{ll}5.50 \text { use } \\ \text { TE } & 303.0 \mathrm{~K}\end{array}$

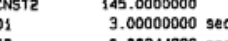

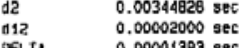

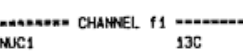

$\begin{array}{ll}\text { MNC1 } & 136 \\ \text { P1 } & 10.94 \text { usec } \\ \text { p2 } & 21,8 \text { use }\end{array}$

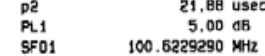

CPDPAG C... CHANEL t2 .........

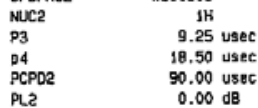

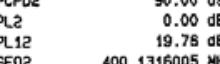

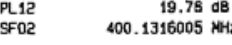

F2 - Processing paraegters

$\begin{array}{lc}\text { SI } & 33768 \\ \text { SF } & 100.6127695 \\ \text { Now } & \text { EHz } \\ \text { SSB } & 0\end{array}$

$\begin{array}{ll}558 & 0 \\ \angle 8 & 1.00 \mathrm{~Hz} \\ \mathrm{FO} & 0 \\ \mathrm{PC} & 1.40\end{array}$

90 NAP plot parsenters

$\begin{array}{lr}\text { CX } & 22.00 \mathrm{~cm} \\ \mathrm{CY} & 8.00 \mathrm{~cm}\end{array}$

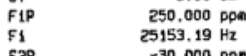

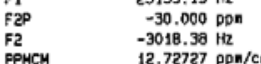

PPHCH
HZCH
$1280.7272512 \mathrm{~Hz} / \mathrm{cm}$

zho-za-214-c1 


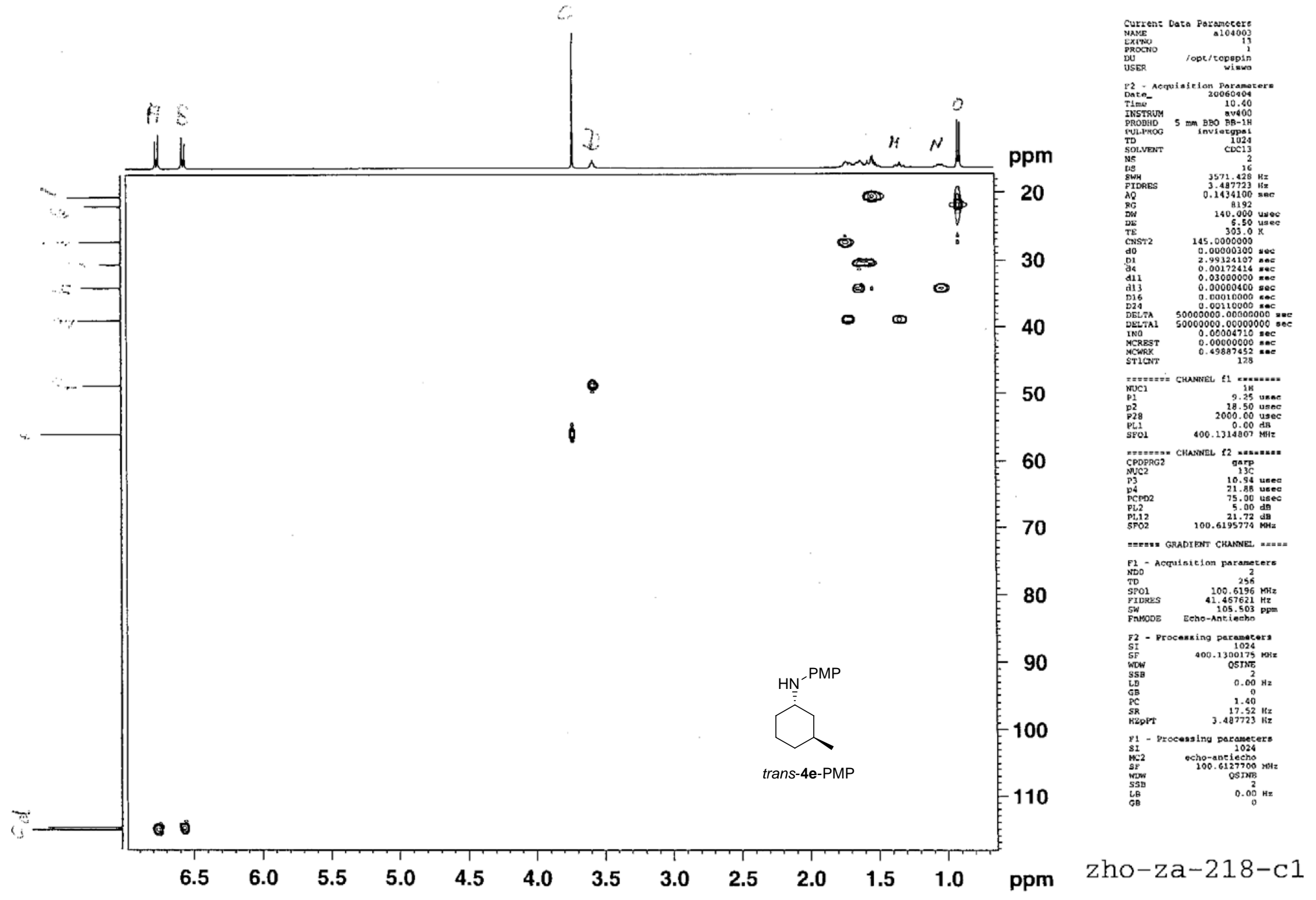




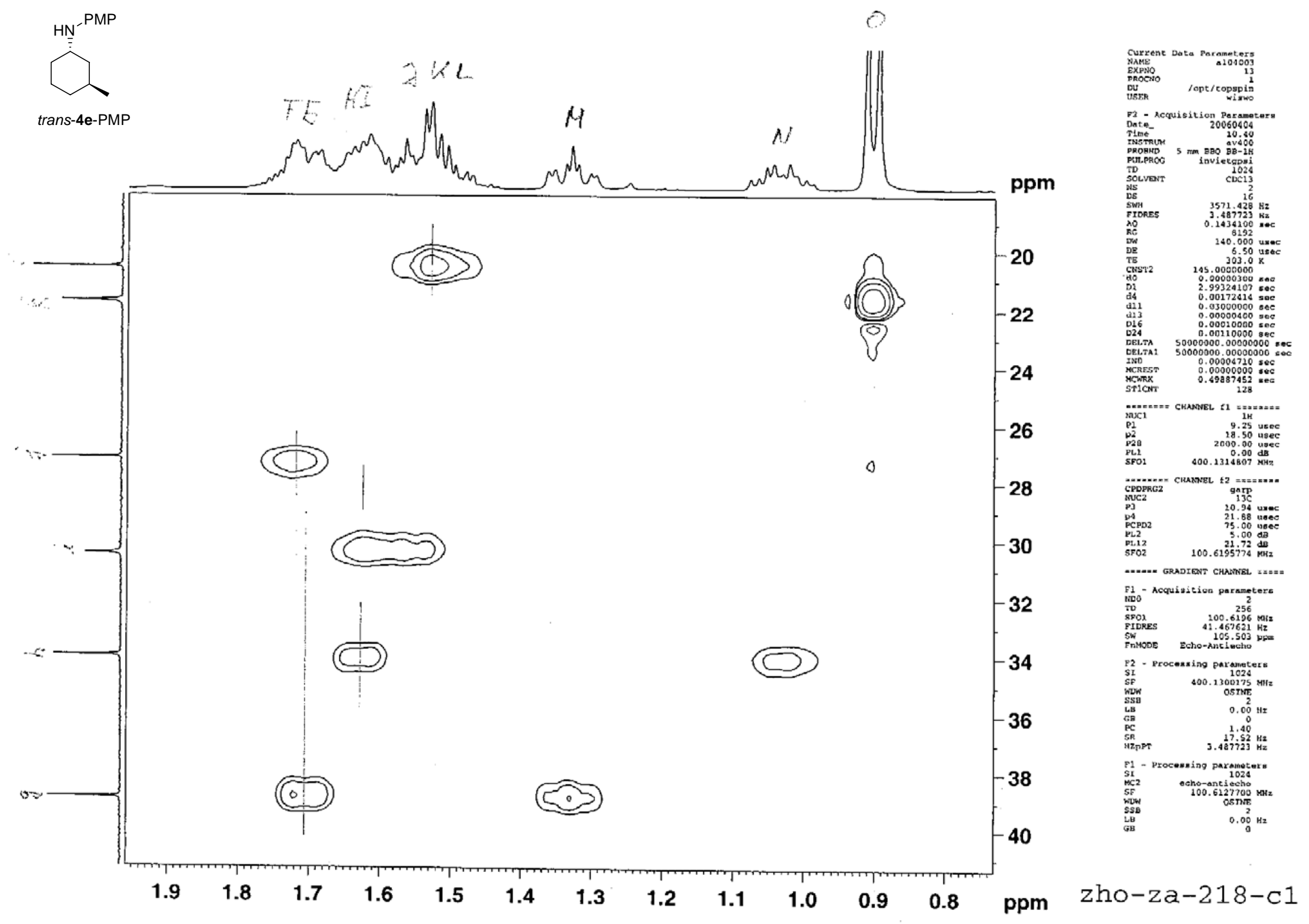




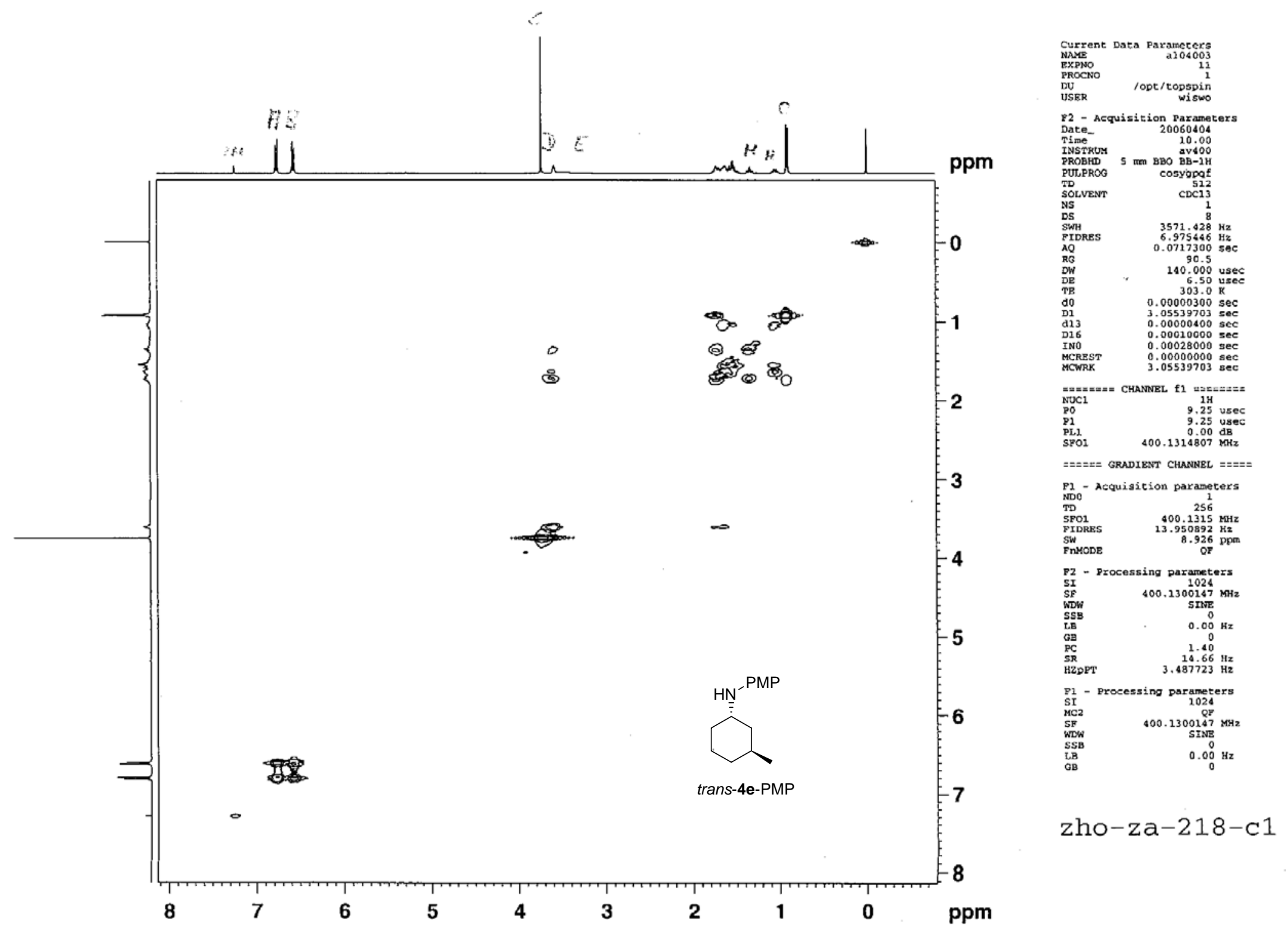

-S 22- 


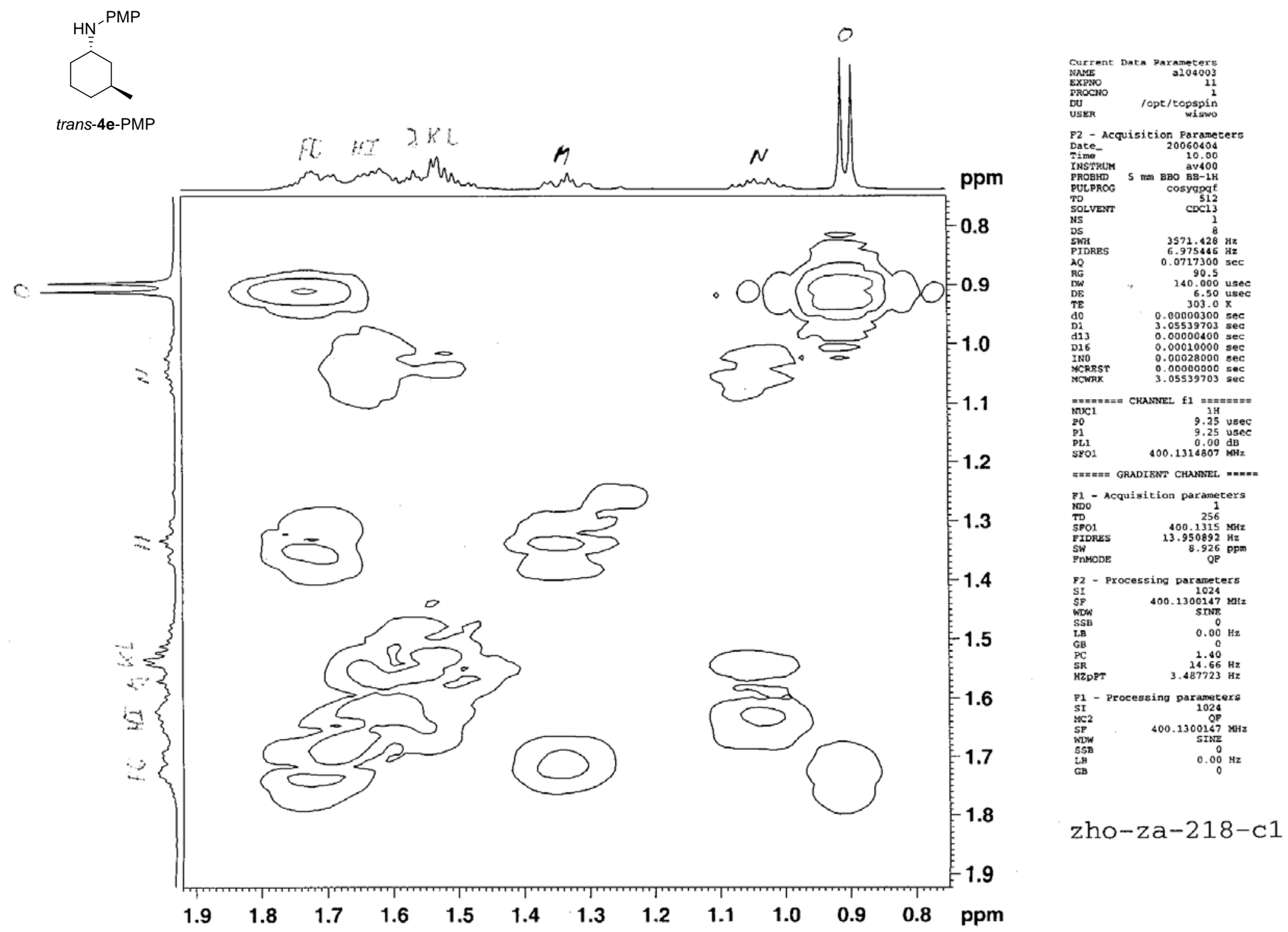



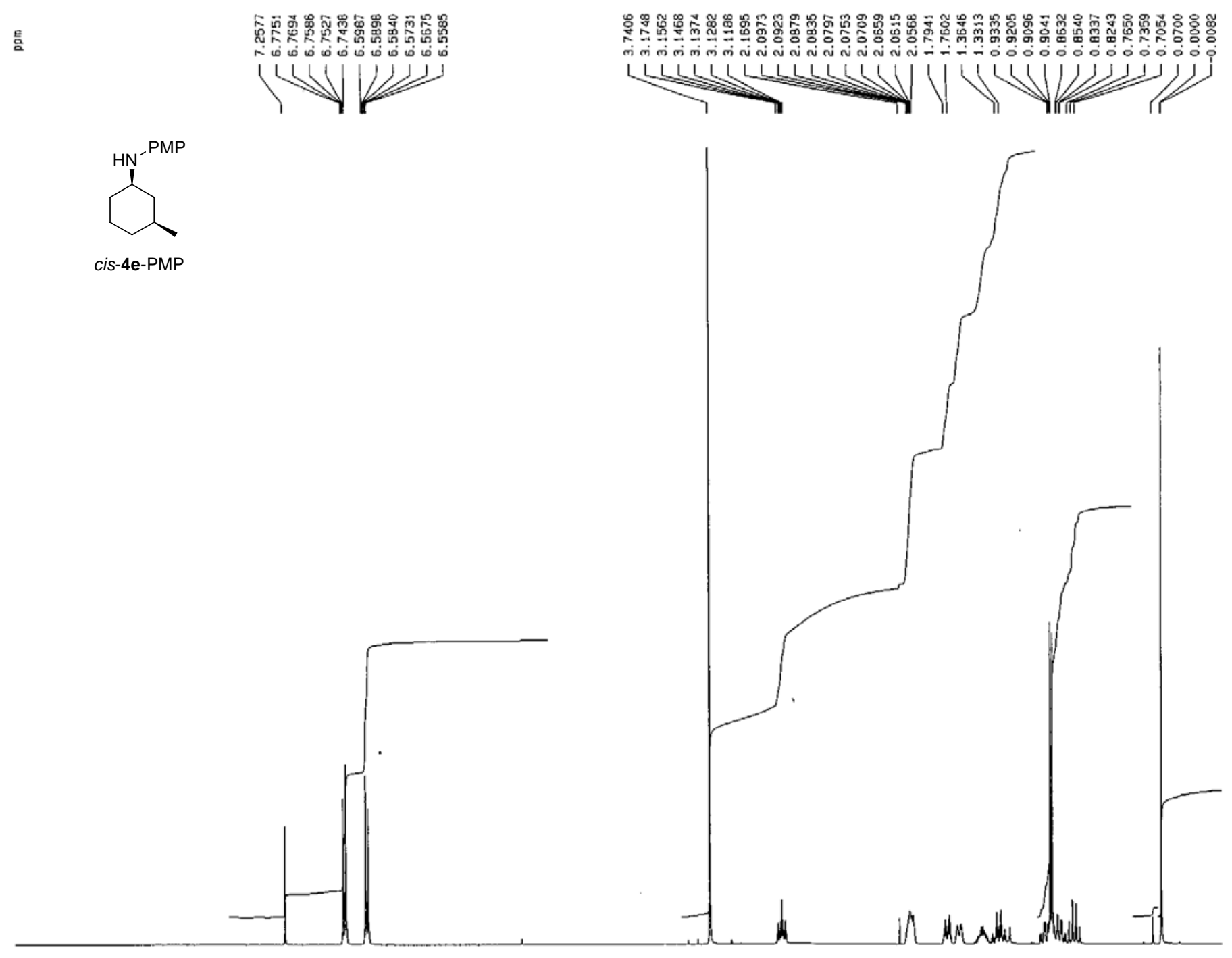

Current Data Parameters
NAYE

EXPNO
PPCCNO

F2 - Acquisition Parameters
Oate_ 20060518

Iime

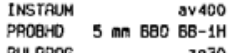

$\begin{array}{lr}2930 \\ \text { PULPROG } & 65535 \\ \text { SOLVENT } & \text { COC13 }\end{array}$

$\begin{array}{lr}\text { NS } & 32 \\ \text { OS } & B\end{array}$

$\begin{array}{ll}\text { SWH } & 8 \\ \text { FHDRES } & \text { B278.145 Hz } \\ \text { FIDES } & 0.126314 \mathrm{~Hz}\end{array}$

$\begin{array}{ll}\text { AO } & 0.126314 \mathrm{~Hz} \\ \mathrm{AO} & 3.9584243 \mathrm{sec}\end{array}$

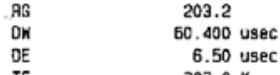

$\begin{array}{ll}\text { TE } & 3.50 \text { use } \\ 01 & 0.0030000 \mathrm{~K}\end{array}$

$=z=z s=z=$ CHANNEL
NUCA

$\begin{array}{lr}\text { NUC1 } & \text { IH } \\ \text { P1 } & 9.25 \text { usec } \\ \text { PL1 } & 0.0008 \\ \text { SF01 } & 400.1324710 \mathrm{HHz}\end{array}$

F2 - Processing paraneters

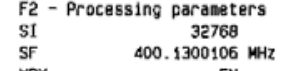

$\begin{array}{cc}\text { SF } & 400.1300106 \mathrm{HHZ} \\ \text { KON } & 0 \mathrm{MH} \\ \text { SSS } & 0 \\ \text { LB } & 0.30 \mathrm{~Hz} \\ \text { GB } & 0\end{array}$

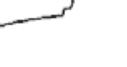

${ }_{c x}^{10}$ NHA plot paraneters 2.00

$\begin{array}{lll}C y & 15.00 \mathrm{~cm} \\ \mathrm{Fip} & 50.502 \mathrm{cos}\end{array}$

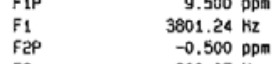

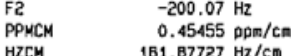

ppm
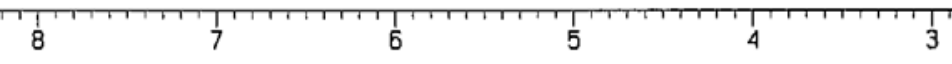

2

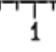

1.

zho-za-266-down 

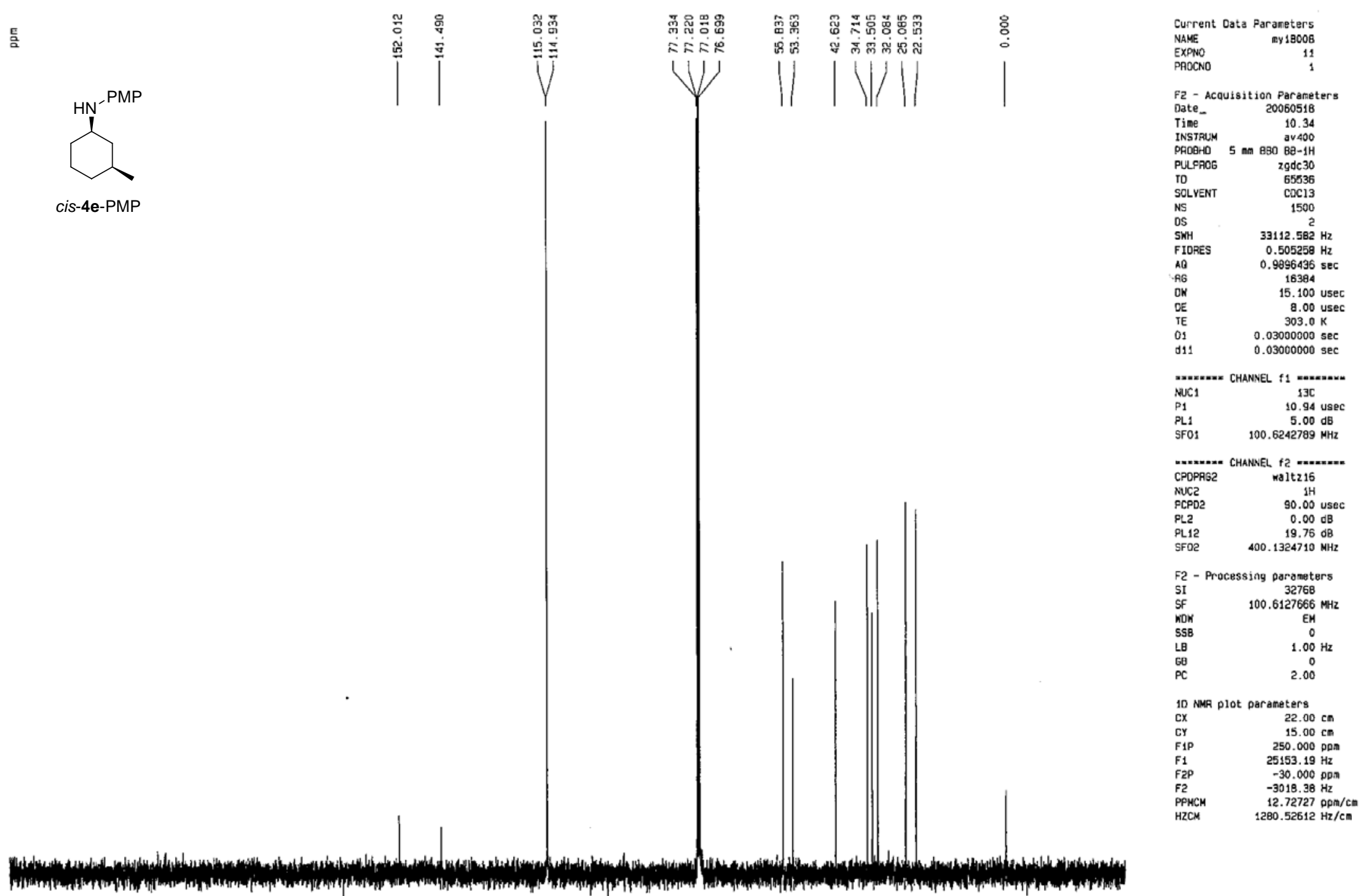

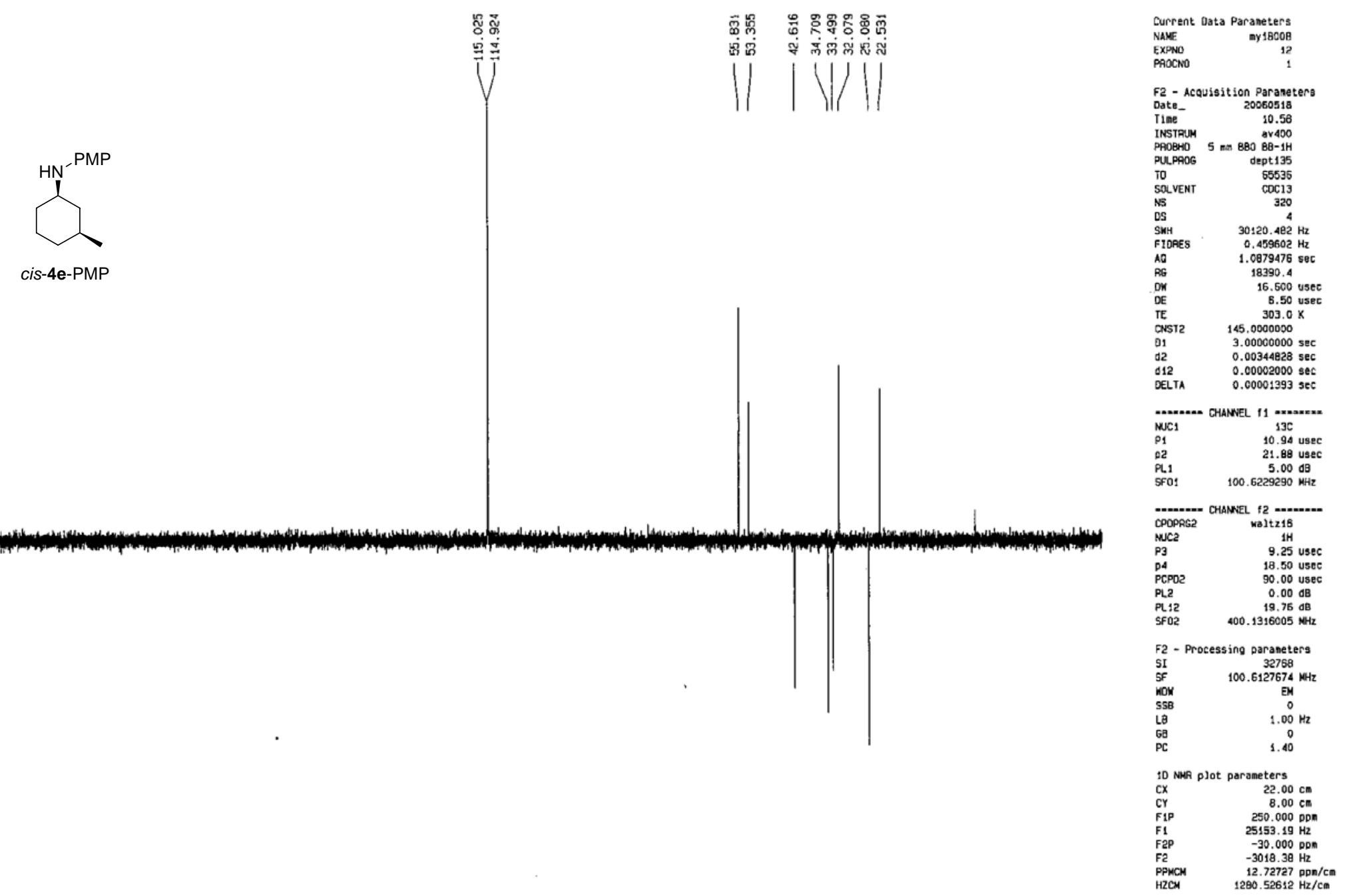
⿷匚⿳亠口冋

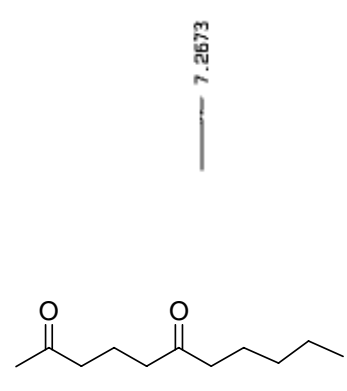

$1 a$

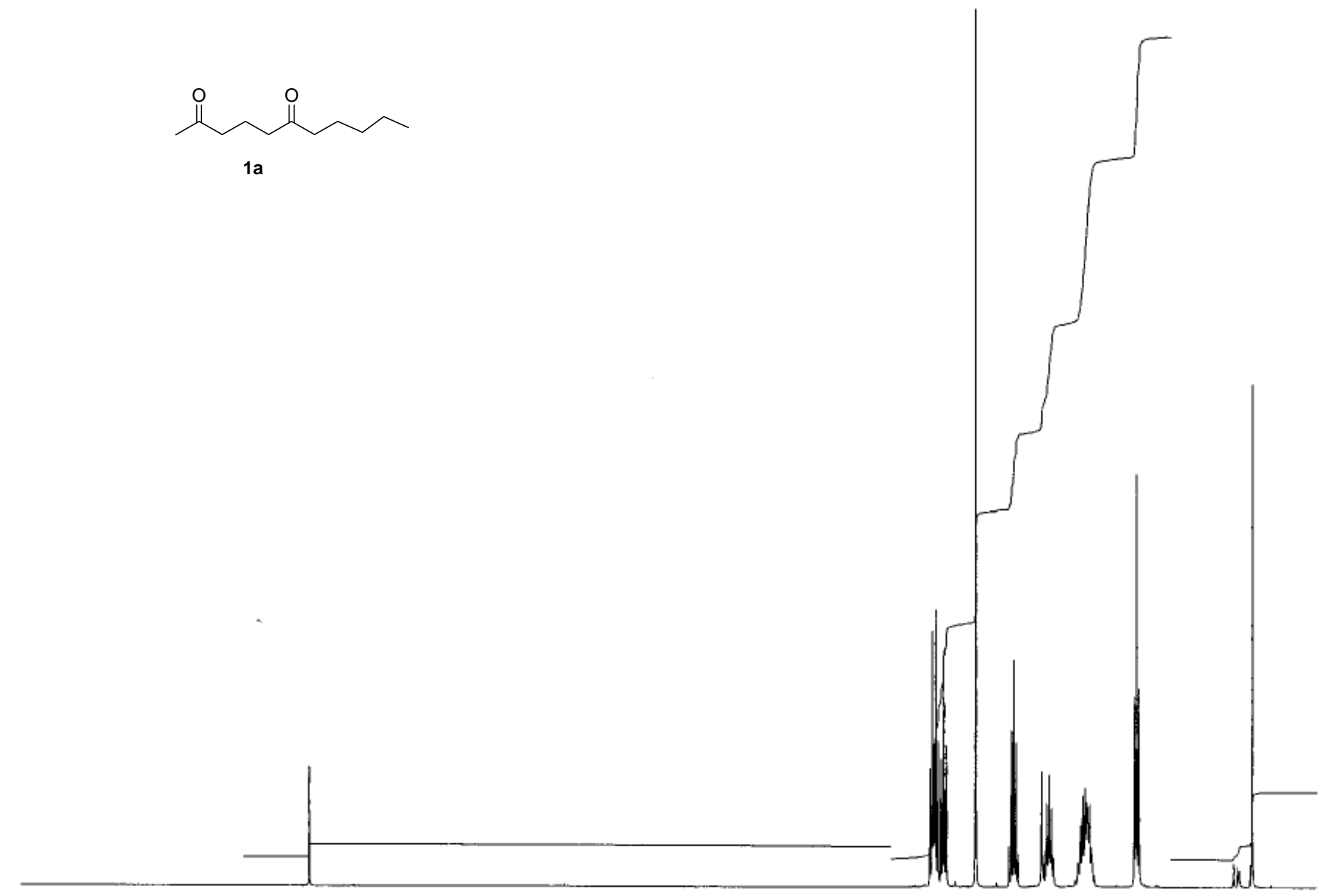

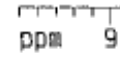

8

11

6

5

4

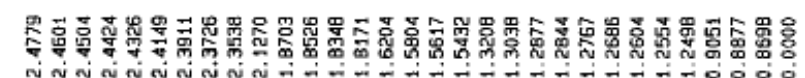

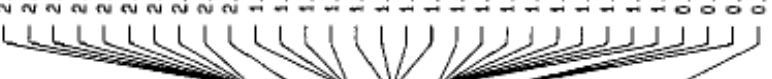

2

11

0
Current Data Paranoters
NAWE Or 17049
ExpNo

EXPNO

F2 - Acquisition Paraneters

Tate

5.38
INSTRUM
av400

Pil PFOG an BEO B8-1H

$\begin{array}{ll} & \begin{array}{l}2930 \\ \text { To }\end{array} \\ \text { Salvent } & 5013 \\ \text { Coc13 }\end{array}$

as 132

$\begin{array}{ll}\text { SWR } & 8278.146 \mathrm{~Hz} \\ \text { FIOAES } & 0.126314 \mathrm{~Hz}\end{array}$

Aa $\quad 3.9584243 \mathrm{sec}$

$\begin{array}{lr}\text { ON } & 60.400 \text { usec } \\ \text { DE } & 6.50 \text { usec } \\ \text { TE } & 303.05 \times\end{array}$

$\begin{array}{ll}0.0303 .0 \mathrm{~K} \\ 01 & 0.00300000 \mathrm{sec}\end{array}$

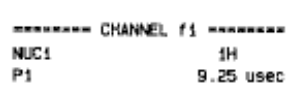

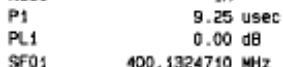

F2 - Processing paranaters

SI $\quad \begin{array}{lll}\text { SI } & \text { 32768 } \\ \text { SF } & 400.1300063 \mathrm{MHz}\end{array}$

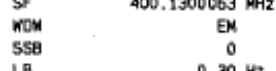

$\begin{array}{ll}\mathrm{GB} & 0 \\ \mathrm{PC} & 2.00\end{array}$

10 NAP plot paraneters

$\begin{array}{ll}\mathrm{CX} & 22.00 \mathrm{~cm} \\ \mathrm{CY} & 15.00 \mathrm{~cm} \\ \mathrm{Fip} & 9.500 \mathrm{pph}\end{array}$

$\begin{array}{ll}F_{1 P} & 9.500 \mathrm{pph} \\ \mathrm{Fi} & 3801.24 \mathrm{~Hz}\end{array}$

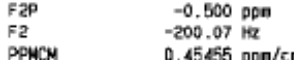

$\begin{array}{ll}\text { PPHCN } & 0.45655 \mathrm{ppm} / \mathrm{cm} \\ \text { HZCM } & 181.07727 \mathrm{~Hz} / \mathrm{cm}\end{array}$

-S 27- 
⿷

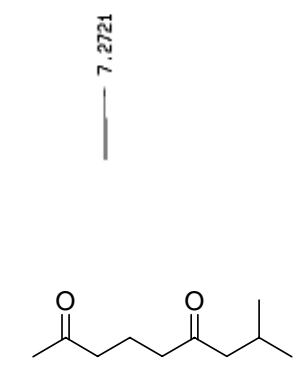

$1 b$

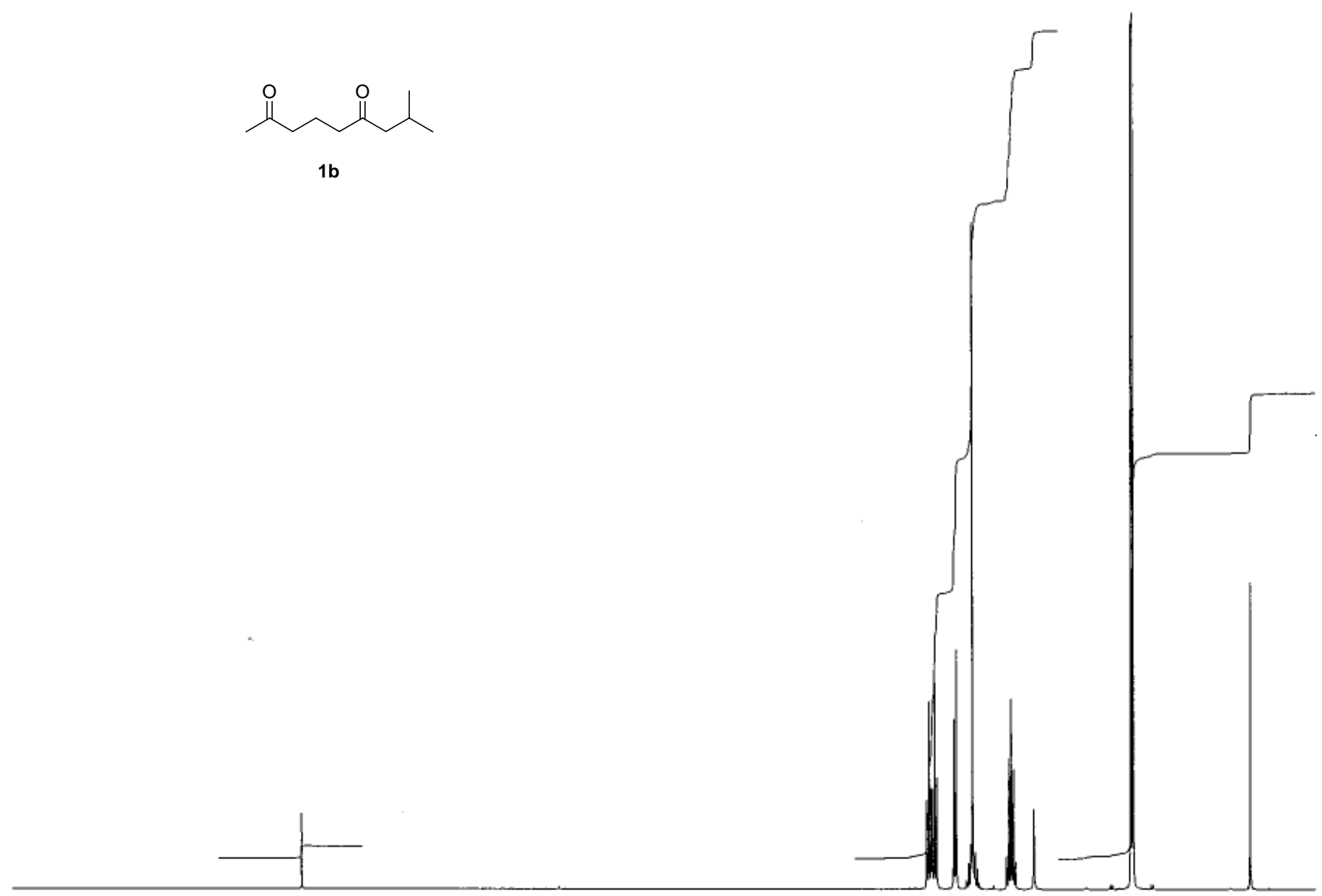

ppm 9

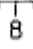

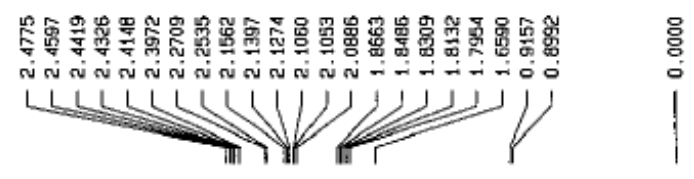

Current dato Paraneters
Sare
ar20057

EXPNO

F2 - Acquisition Paraneters

F2 - Acquisition Paraneters
Date_ 20061021
Tane

Tine

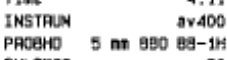

2990
PU. POPOG

$\begin{array}{lr}\text { NS } & 32 \\ \text { DS } & \text { B }\end{array}$

$\begin{array}{ll}\text { FIDFES } & \text { B278. } 146 \mathrm{~Hz} \\ 0.126314 \mathrm{~Hz}\end{array}$

$\begin{array}{ll}\text { A0 } & 3.9584243 \mathrm{sec} \\ \text { RG } & 114\end{array}$

60,400 usec
DE

$0.00300000 \mathrm{stc}$

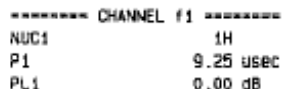

$\begin{array}{lr}\text { PL1 } & 9.00 \mathrm{~dB} \\ \text { SFO1 } & 400.1324710 \mathrm{NHz}\end{array}$

Fa - Processing parameters
SI
32760

$\begin{array}{ll}\text { SI } & 32760 \\ \text { SF } & 400.1300045 \\ \text { NOW } & \text { HHZ }\end{array}$

$\begin{array}{lc}\text { SSB } & 0 \\ \text { LB } & 0.30 \mathrm{~Hz} \\ G B & 0.00\end{array}$

10 NWA plot parsneters

$\begin{array}{ll}\mathrm{CX} & 22.00 \mathrm{~cm} \\ \mathrm{Cr} & 15.00 \mathrm{~cm} \\ \mathrm{~F} & 9.500 \mathrm{com}\end{array}$

$\begin{array}{ll}\mathrm{F} 1 & 9.500 \mathrm{PDO} \\ \mathrm{F} 2 \mathrm{PO} & -0.24 \mathrm{~Hz}\end{array}$

FP $\quad-200.07 \mathrm{~Hz}$

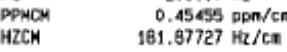



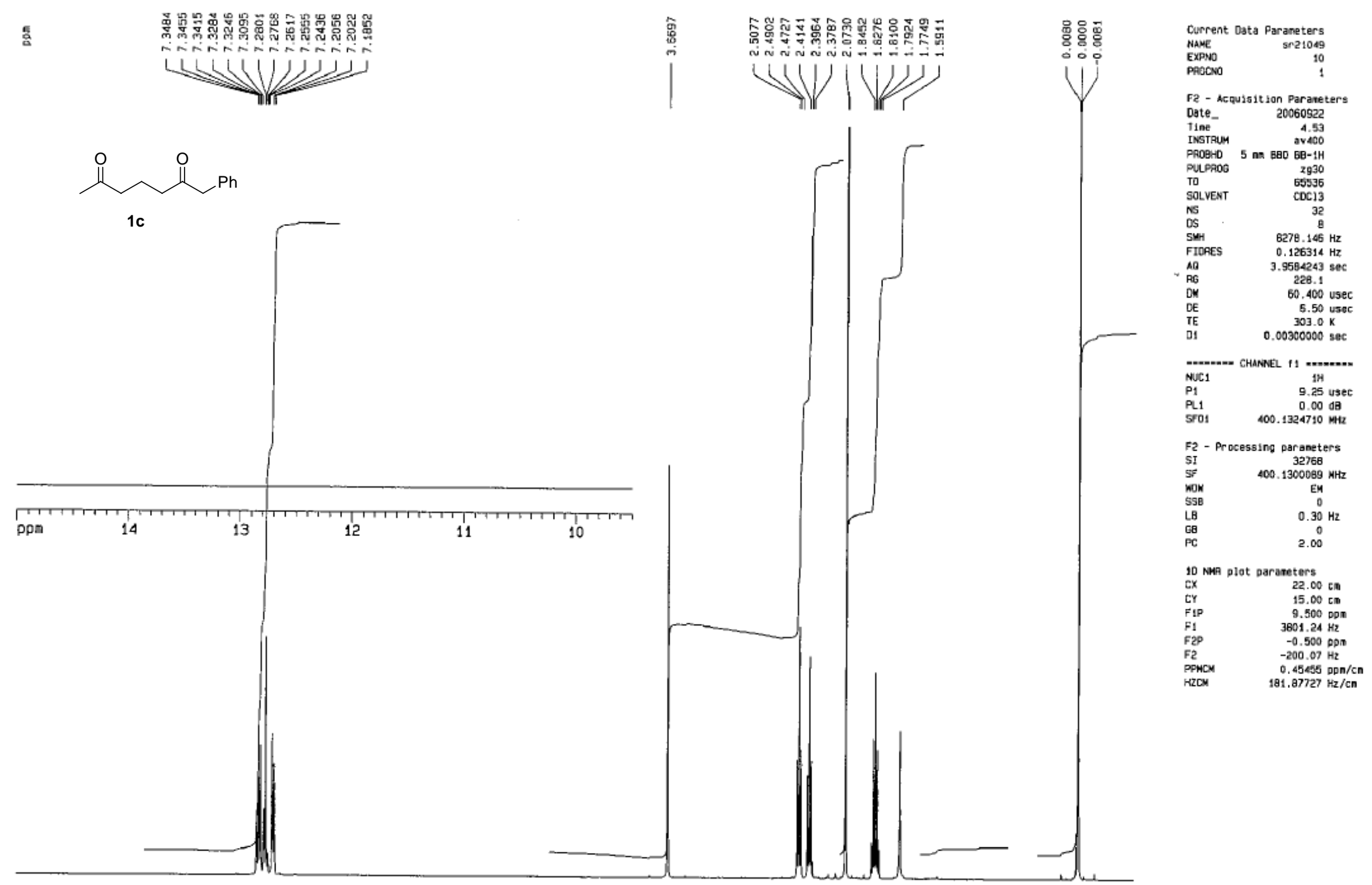

ppe 9

8

6

5

${ }_{4}^{11}$

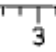

2

1

The

zho-za-462-re-2

-S 29- 

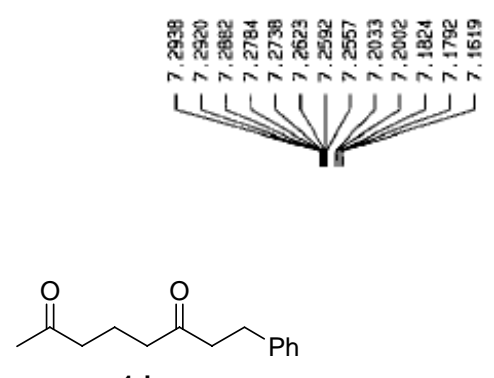

1d

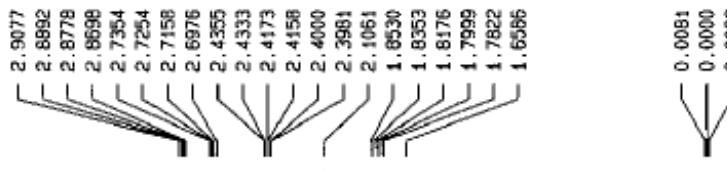

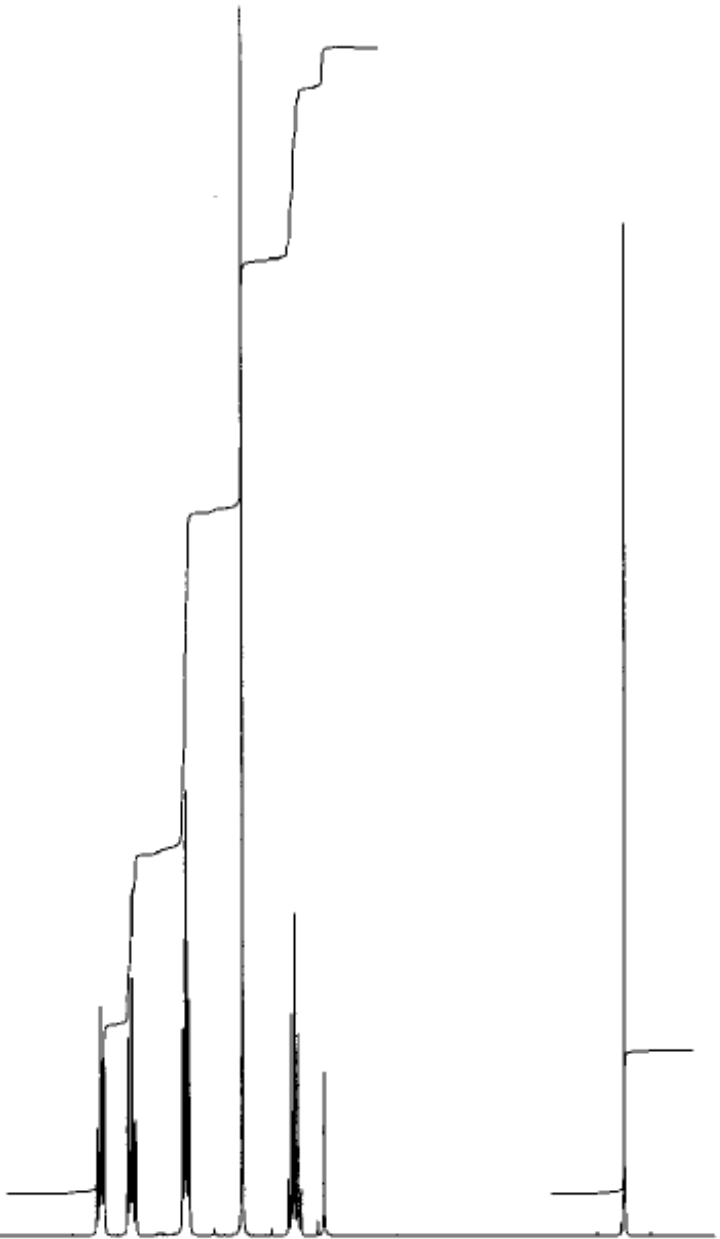

Current Data Paraneters
NaMe
Sr21012

EXPEO

F2 - Acquisition Paraneters

$\begin{array}{lr}\text { Date_- } & \text { 20060921 } \\ \text { Tine } & 13.15\end{array}$

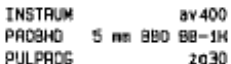

2930
TD TDAOG
SOLIVENT

\begin{tabular}{lr} 
NS & Cocla \\
OS & 32 \\
\hline
\end{tabular}

SWH $8278.196 \mathrm{~Hz}$

AQ $\quad 3.9554243 \mathrm{gec}$

on $\quad 60.400$ usec

$\mathrm{TE}-6.50 \mathrm{Us}$ $0.00300000 \mathrm{sec}$ NUC1

PL1 $\quad 0.00 \mathrm{~dB}$

F2 - Processing paraneters

SI $\quad 400,1300006 \mathrm{HHz}$

$\begin{array}{lc}\text { NOW } & \text { EM } \\ \text { SSB } & 0 \\ \text { LB } & 0.30 \mathrm{~Hz} \\ \text { GB } & 0\end{array}$

10 NuF plot paranaters

$\begin{array}{ll}\mathrm{cX} & 22.00 \mathrm{~cm} \\ \mathrm{cY} & 15.00 \mathrm{cn}\end{array}$

$\begin{array}{ll}\text { F1P } & 9.500 \\ \text { F1 } & 30001.24 \mathrm{~Hz} \\ \mathrm{Fr} & \end{array}$

F2 $\quad-200.07 \mathrm{~Hz}$

$\begin{array}{ll} & 0.456459 \mathrm{ppn} / \mathrm{cm} \\ \mathrm{HZCH} & 181.87727 \mathrm{~Hz} / \mathrm{Ca}\end{array}$ 

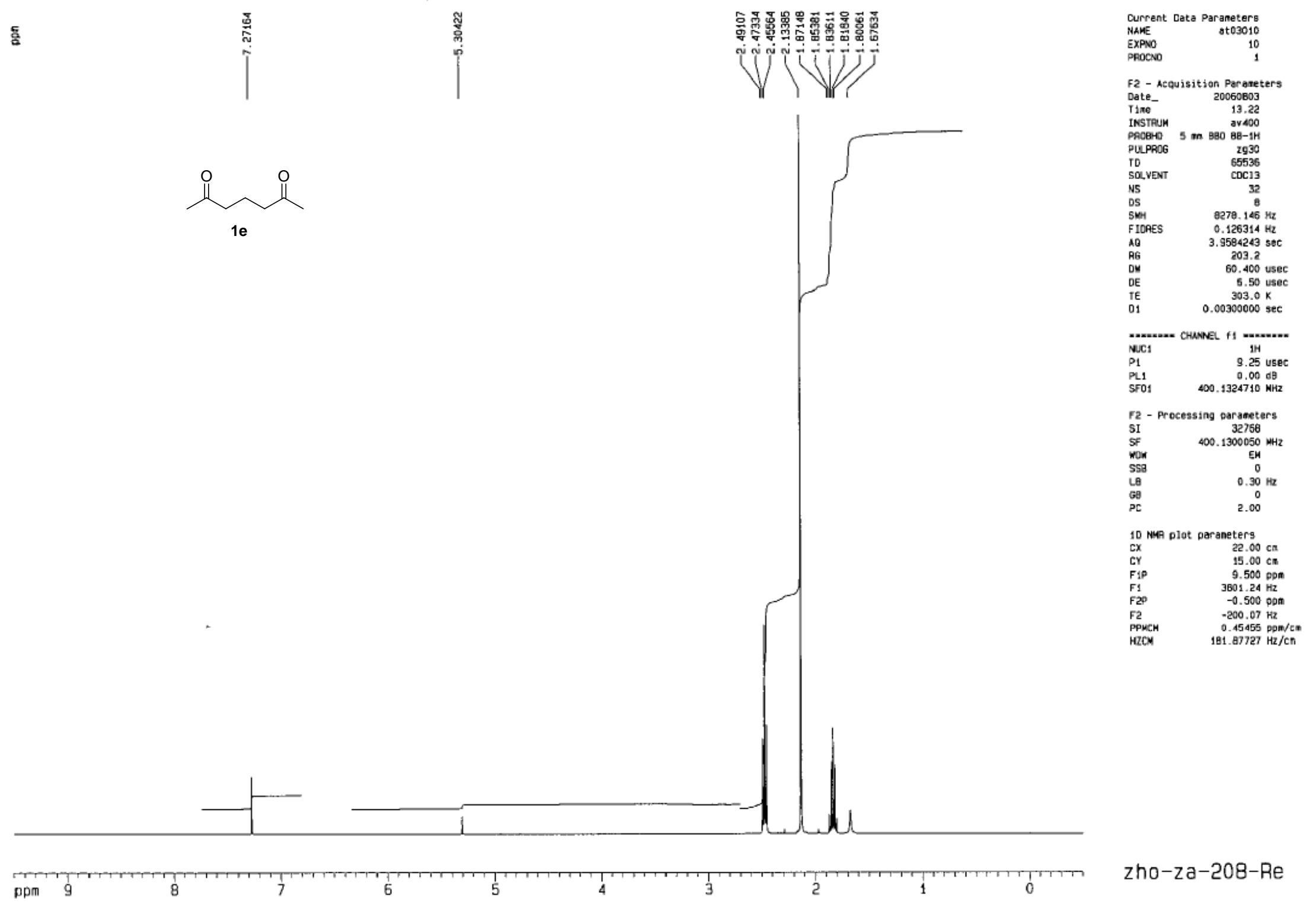

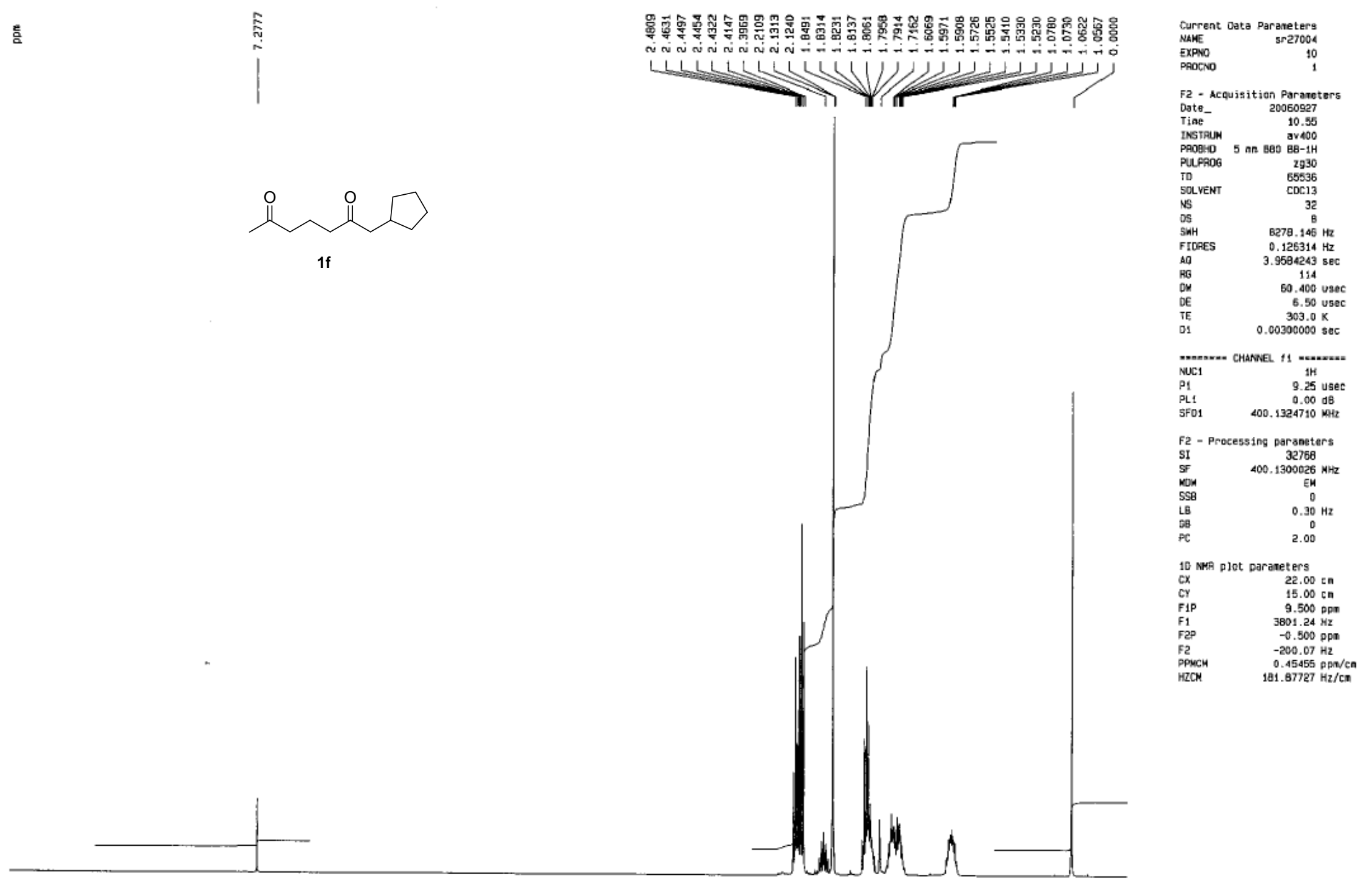

ppm
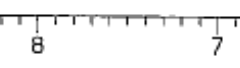

6

5

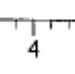




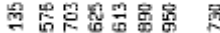

कुष

U1HII
1

'T

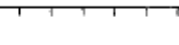

100
50
Current Data Paraneters

EXPEO $\quad 5 r 27004$

F2 - Acquisitition Paraneters

The 11.23

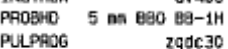

PgdC 30
TD
TD

$\begin{array}{lr}\text { NS } & 1500 \\ \text { OS } & 2\end{array}$

SWH $\quad 33112.52 \mathrm{Z} 2 \mathrm{~Hz}$

$\begin{array}{ll}A B & 0.9896436 \mathrm{sec} \\ 96 & 1890.4\end{array}$

.96
of

15.100 usec
8.00 usec
$303.0 \mathrm{~K}$

$3.00 \mathrm{usec}$
$303.0 \mathrm{~K}$
$.03000000 \mathrm{sec}$

$\begin{array}{ll} & 0.03000000 \mathrm{sec} \\ \text { d11 } & 0.03000000 \mathrm{sec}\end{array}$

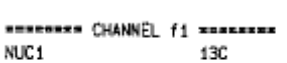

PUt $\quad 10.94$ usec

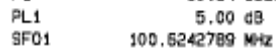

CPOPAS2 CHANAEL fa nus......

$\begin{array}{lc}\text { CPOPAG } & \text { moltz16 } \\ \text { NCL2 } & 1 \mathrm{H} \\ \text { PCPOa } & 90.00 \text { use }\end{array}$

PL2 2

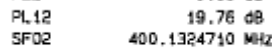

F2 - Processing paremeters

$\begin{array}{ll}\text { SI } & 32769 \\ \text { SF } & 100.6127651 \\ \text { MHI }\end{array}$

$\begin{array}{lc}\text { SF } & 100.6127651 \\ \text { NOW } & \text { EM } \\ \text { SSB } & 0 \\ \text { LB } & \text { S }\end{array}$

$\begin{array}{ll}\mathrm{LB} & 1.00 \\ \mathrm{~GB} & 0 \\ \mathrm{PC} & 2.00\end{array}$

10 NUP Dlot paraneters

$\begin{array}{ll}\mathrm{CX} & 22.00 \mathrm{cR} \\ \mathrm{CY} & 15.00 \mathrm{cE}\end{array}$

$\begin{array}{ll}\text { F1P } & 250.000 \mathrm{ppn} \\ \text { F1 } & 25153.19 \mathrm{~Hz}\end{array}$

F2 $\quad-3018.38 \mathrm{~Hz}$

$\begin{array}{lr}\text { PPHCM } & 12.72727 \\ \mathrm{HZCM} & 1280.52612 \mathrm{pr} / \mathrm{cm}\end{array}$ 


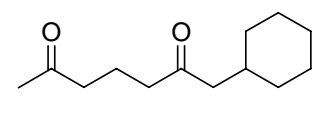

$1 \mathrm{~g}$

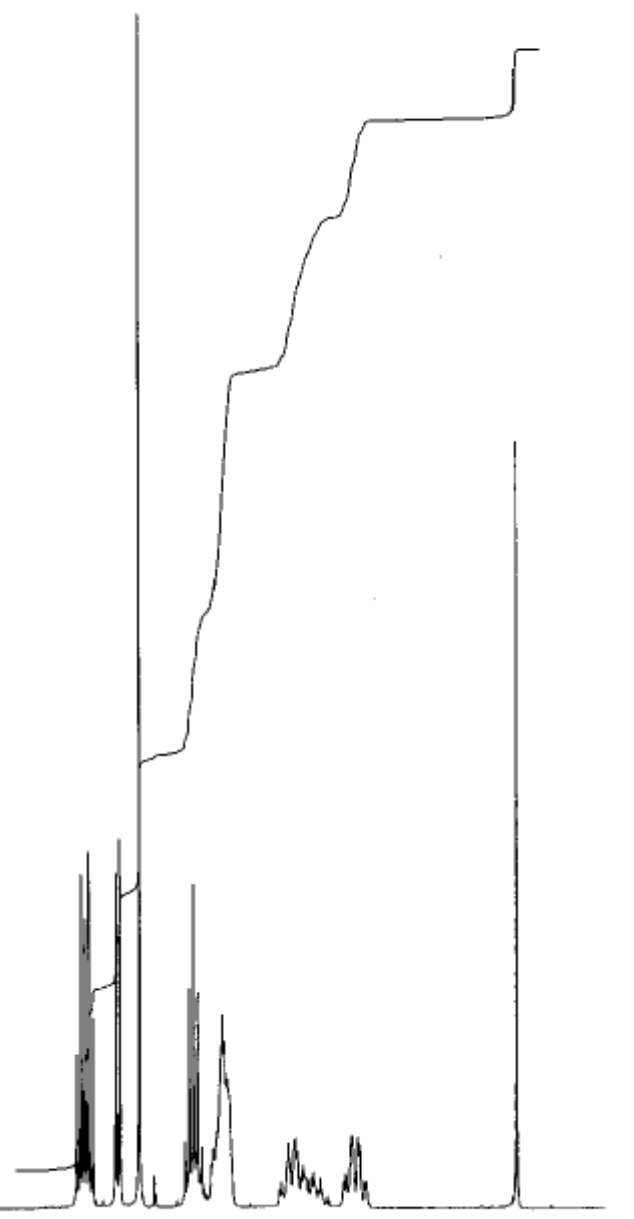
10

F2 - Acquisition Parameters

Date

Date 20060823

INSTRIN $\frac{d .05}{d p \times 300}$

PROEHD $5 \mathrm{~mm}$ QNP $1 \mathrm{H} /$

2930
PULPGOG
TO

NS $\quad 35$

DS 0172.00

SNH $\quad \begin{array}{ll}6172.839 \mathrm{~Hz} \\ \text { FIDRES } & 0.188380 \mathrm{~Hz}\end{array}$

$\begin{array}{ll}\text { FIDRES } & 0.188380 \mathrm{~Hz} \\ A Q & 2.6542580 \mathrm{sec}\end{array}$

RG 143.7

DE $\quad 81.000$ usec

4.50 usec

$01 \quad 2.00000000 \mathrm{sec}$

$==z=z==$ CHANEL
NUC1 $1=z=x=z=x$

$\begin{array}{ll}\text { NUC1 } & 1 \mathrm{H} \\ \mathrm{P} 1 & 7.56 \text { usec }\end{array}$

$\begin{array}{lr}\text { PL1 } & -6.00 \mathrm{~dB} \\ \text { SF01 } & 300.1318534 \mathrm{MHz}\end{array}$

F2 - Processing paraneters

SI 16384

$\begin{array}{lc}\text { SF } & 300.1300006 \text { MH } \\ \text { WDW } & \text { EM }\end{array}$

$\begin{array}{ll}\text { SSB } & 0 \\ \text { LB } & 0.30 \mathrm{~Hz}\end{array}$

$\begin{array}{lr}68 & 0 \\ \mathrm{PC} & 2.00\end{array}$

10 Mur plot paraseters

CX $\quad 22.00 \mathrm{~cm}$

F 1 P $\quad 9.500$ pon

F1 $2851.24 \mathrm{~Hz}$

$\begin{array}{ll}F 2 P & -0.500 \mathrm{ppe} \\ F 2 & -150.07 \mathrm{~Hz}\end{array}$

$0.45455 \mathrm{ppe} / \mathrm{cm}$

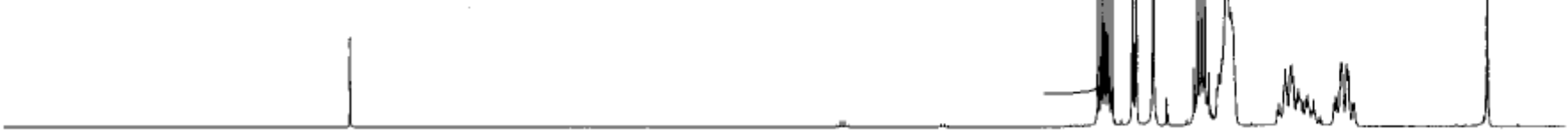


要
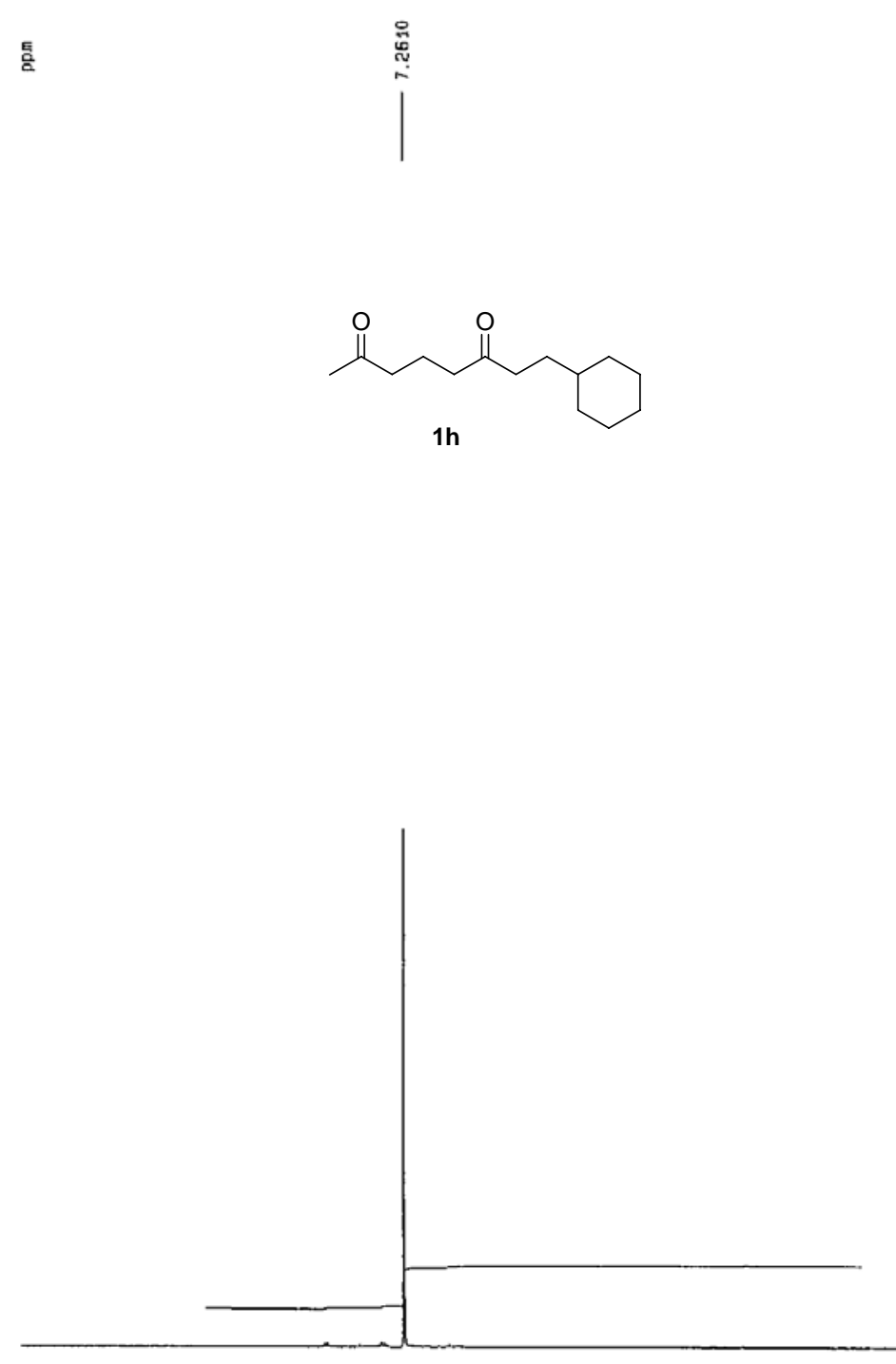

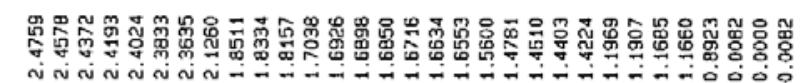
UnN

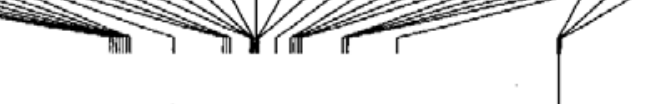

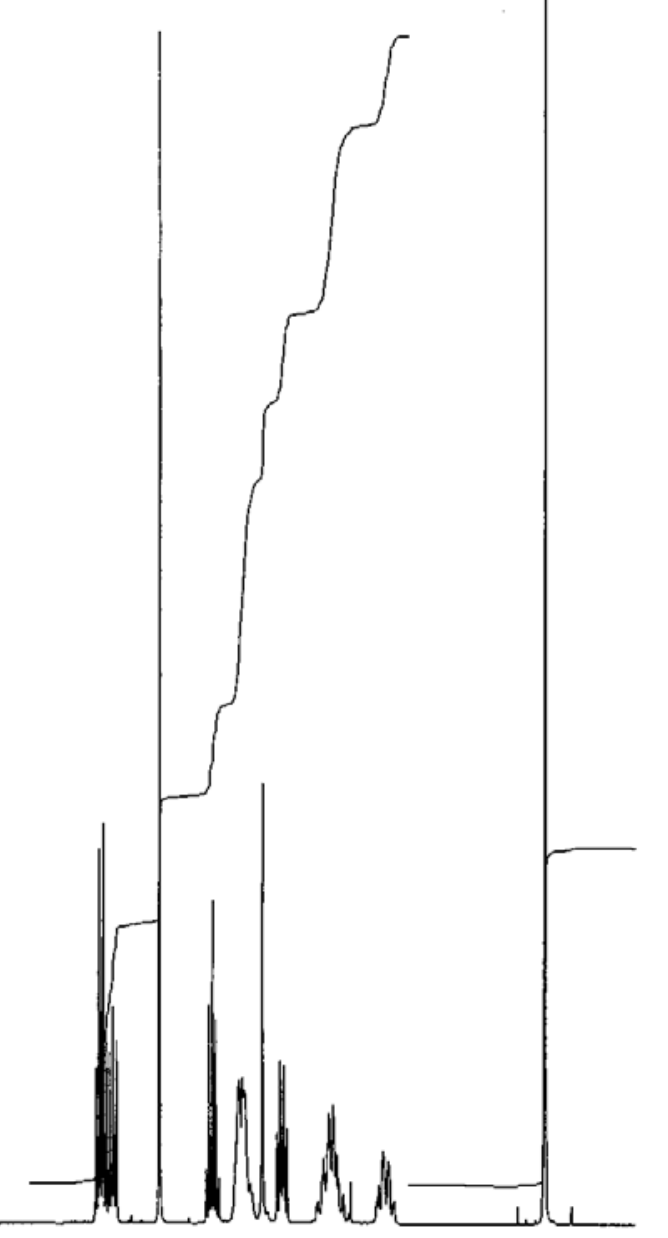

T)
Current Data Parameters

$\begin{array}{lr}\text { NANE } & \text { Or } 31012 \\ \text { EXPNO } & 10 \\ \text { PROCNO } & 1\end{array}$

Fe - Acquisition Parameters

$\begin{array}{lr}\text { Date } & 20061031 \\ \text { Iime } & 12.48 \\ \text { InSTRUM } & \text { ava00 }\end{array}$ INSTRUM
PAOSHO $5 \mathrm{~mm}$ BBO BV $B \mathrm{AO}-1 \mathrm{H}$

$\begin{array}{lr}\text { PULPAOG } & 2930 \\ \text { TO } & 65536 \\ \text { SOLVENT } & \text { COC13 }\end{array}$

$\begin{array}{lr}\text { NS } & 32 \\ \text { DS } & 8\end{array}$

$\begin{array}{ll}\text { SWH } & 8278.145 \mathrm{~Hz} \\ \text { FIDAES } & 0.126314 \mathrm{~Hz}\end{array}$

$\begin{array}{ll}A 0 & 0.126314 \mathrm{mz} \\ A G & 958243 \mathrm{sec}\end{array}$

$\begin{array}{rr}\text { OH } & 50.400 \mathrm{usec} \\ \mathrm{OH} & 6.50 \mathrm{usec} \\ \mathrm{OE} & 3030 \mathrm{~K}\end{array}$

$\begin{array}{lll}01 & 303.0 \mathrm{~K} \\ 0 & 0.00300000 \mathrm{sec}\end{array}$

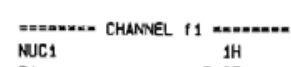

$\begin{array}{ll}\text { NUC1 } & \text { H } \\ P_{1} & 9.25 \mathrm{usec}\end{array}$

$\begin{array}{ll}P L 1 & 0.00 \mathrm{~dB} \\ \mathrm{SFOS} & 400.1324710 \mathrm{KHz}\end{array}$

F2 - Process ing parameters
SI
32768

$\begin{array}{lc}\text { SI } & 32768 \\ \text { SF } & 400.1300069 \mathrm{MHz} \\ \text { KON } & \text { EM }\end{array}$

$\begin{array}{ll}\mathrm{LS} & 0.30 \mathrm{~Hz} \\ \mathrm{HB} & 2.0 \\ \mathrm{PC} & 2.00\end{array}$

10 NHA Dlot Dorsmeters

$\begin{array}{ll}\mathrm{CX} & 22.00 \mathrm{~cm} \\ \mathrm{CY} & 15.00 \mathrm{cs}\end{array}$

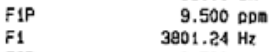

$\begin{array}{ll}\mathrm{FPP} & -0.500 \mathrm{ppm} \\ \mathrm{FP} & -2007 \mathrm{~Hz}\end{array}$

$\begin{array}{lr}\text { PPHCM } & -200.07 \mathrm{~Hz} \\ \mathrm{HZCM} & 181.85775 \mathrm{ppm} / \mathrm{cm}\end{array}$ 


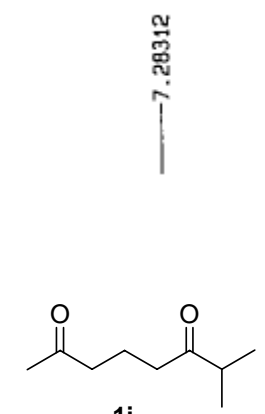

$1 \mathrm{i}$

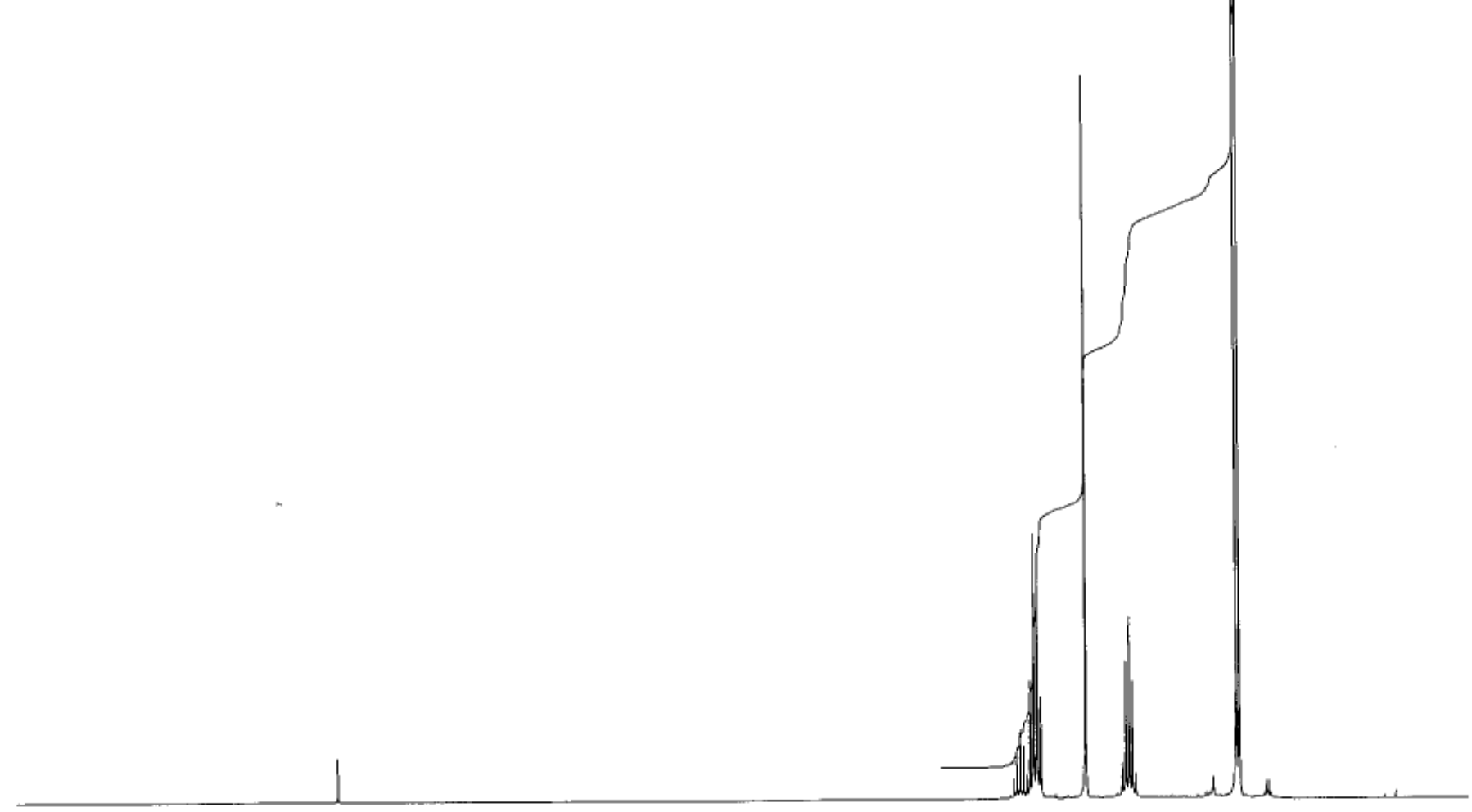

ppm 9

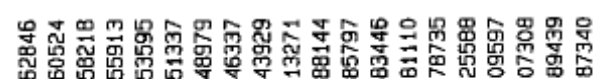

ชัง

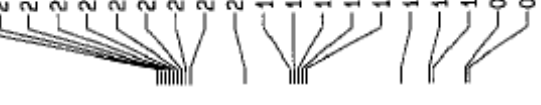

-

$B$

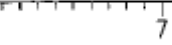

6

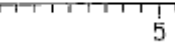

Current Data Paraneters

EXPNO

PACCNO 21006

F2 - ACquisition Parareters

Date_ 20060821

10.27
$90 \times 300$

PPOBHD $5 \mathrm{~mm}$ ONP $1 \mathrm{H} /$

PULPROG zg30

$\begin{array}{ll}\text { TD } & 32768 \\ \text { SOLVENT } & \text { CDC13 }\end{array}$

$\begin{array}{lr}\text { NS } & 32 \\ \text { OS } & 0\end{array}$

OS $\quad 6172.839 \mathrm{~Hz}$

FIDAES $\quad 0.188380 \mathrm{~Hz}$

FG $\quad 181$

$\begin{array}{lr}81.000 \text { usec } \\ \text { DE } & 4.50 \text { usec }\end{array}$

$2120000000 \mathrm{sec}$

$== \pm====$ CHANEL
NUC1

$\begin{array}{lr}\text { P1 } & 7.55 \text { usec } \\ \text { PL1 } & -6.00 \mathrm{~dB}\end{array}$

$\begin{array}{lr}\text { PL1 } & -6.00 \mathrm{~dB} \\ \text { SF01 } & 300.1318534\end{array}$

F2 - Process ing paraneters

SI Procosons prameters

SF $\quad 300.1299994 \mathrm{MHz}$

NDK EM

$\begin{array}{lc}\text { SSB } & 0 \\ L B & 0.30 \mathrm{~Hz} \\ 6 \mathrm{G} & 0\end{array}$

10 NAF plot parameters 22.00

$\begin{array}{ll}\text { CX } & 22.00 \mathrm{~cm} \\ \mathrm{CY} & 15.00 \mathrm{~cm}\end{array}$

F1p $\quad 9.500 \mathrm{ppn}$

$\begin{array}{ll}F_{1} & 2851.24 \mathrm{~Hz} \\ \text { F } & -0.500\end{array}$

$\begin{array}{ll} & -0.500 \mathrm{pPm} \\ \mathrm{FP} & -150.07 \mathrm{~Hz}\end{array}$

$\begin{array}{ll}\text { PPMCM } & 0.45455 \mathrm{ppm} / \mathrm{cm} \\ \mathrm{HZCM} & 136.42273 \mathrm{~Hz} / \mathrm{Ca}\end{array}$

zho-za-378 


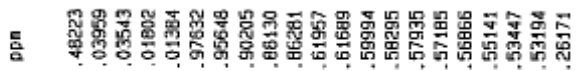

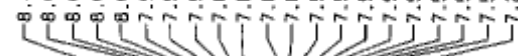
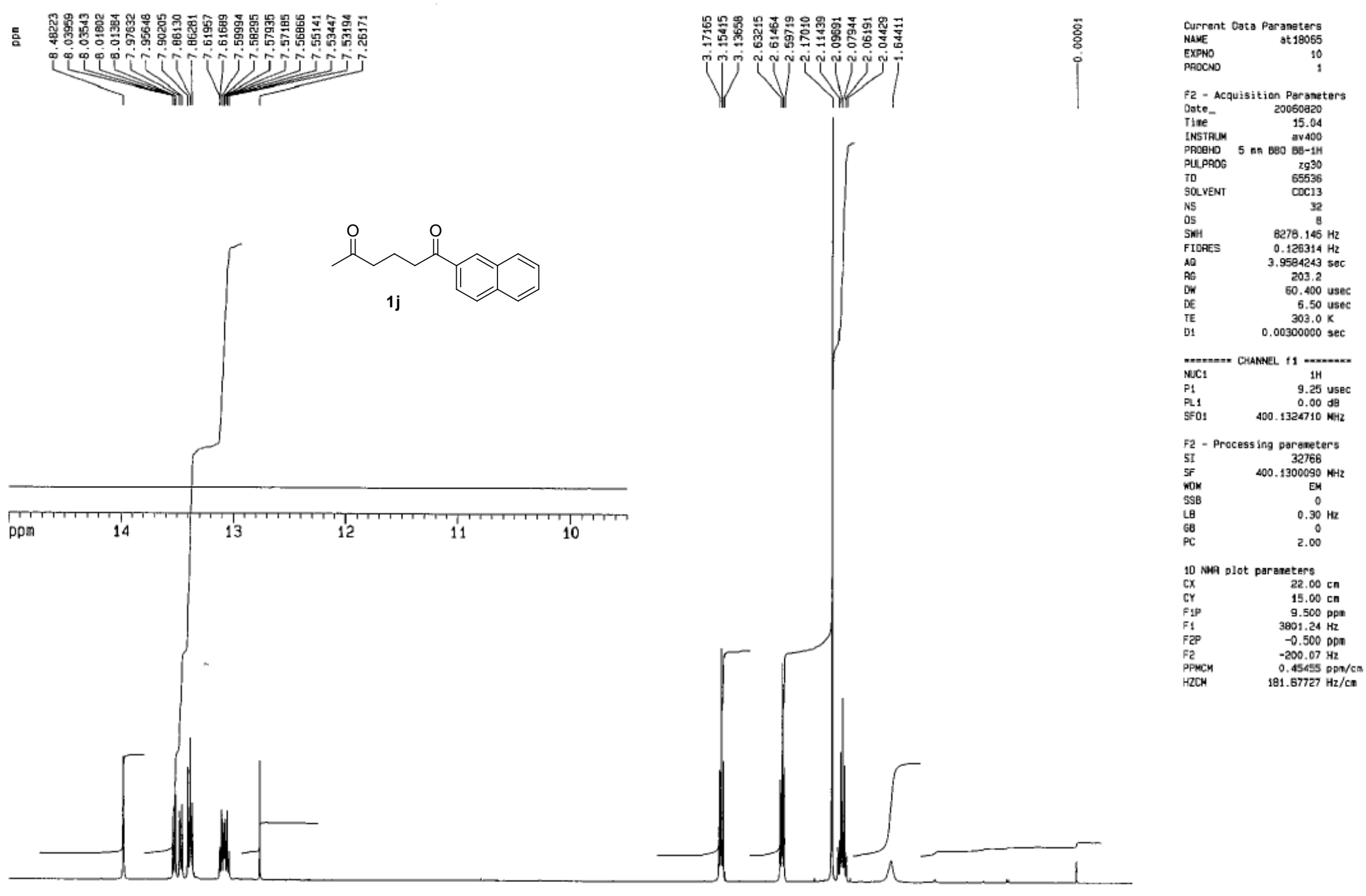
$7727 \mathrm{~Hz} / \mathrm{Cn}$
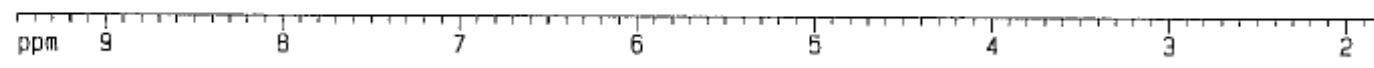

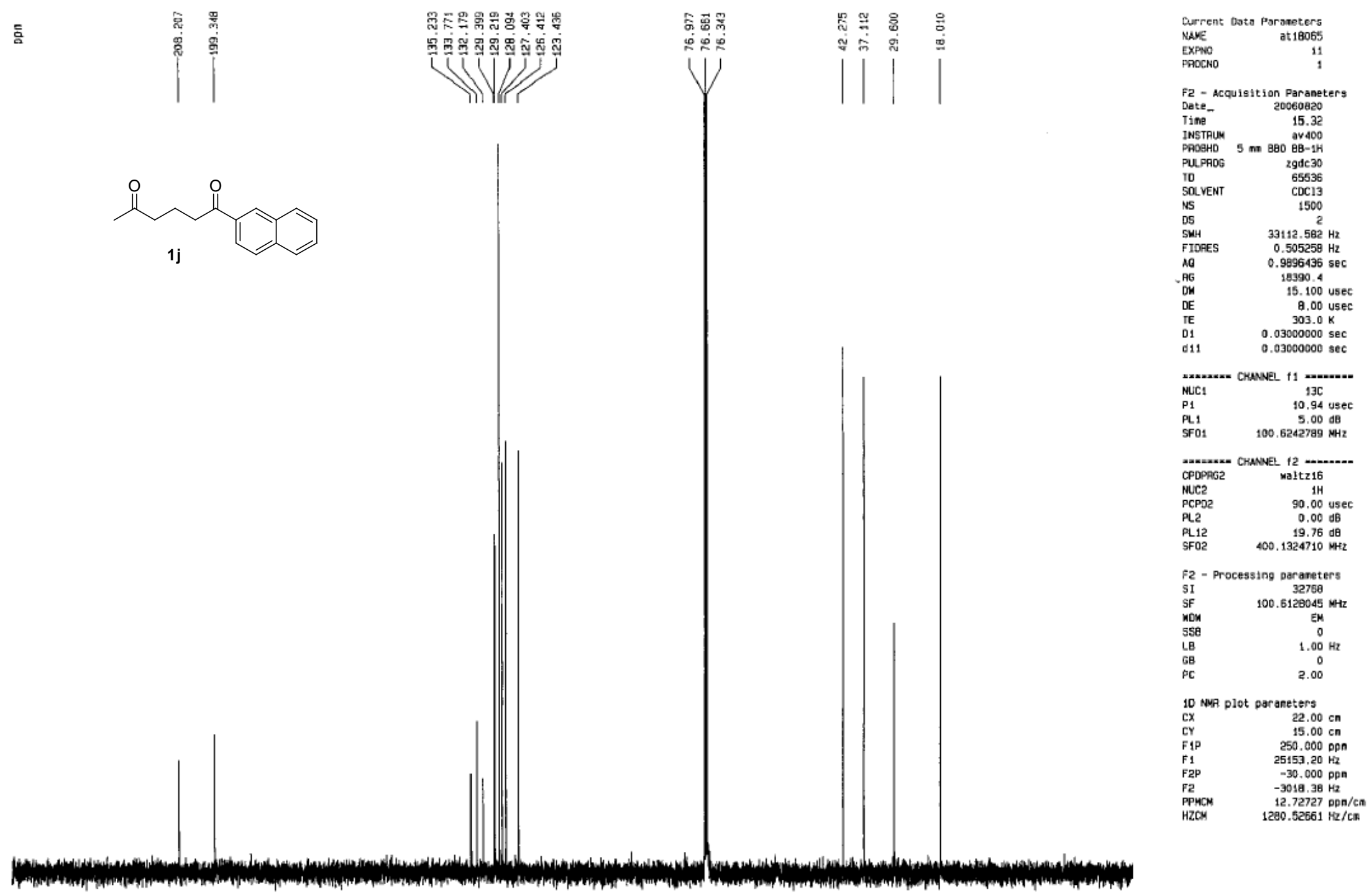
current cata Pararater:

RARE

FrOCNO 4095
10
1

F2 - Acqussition Parareters

20070215

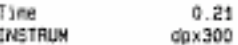

MAEHD $5 \mathrm{ma}$ OSP IH/

$\begin{array}{lr}\text { PUCFROG } & 3990 \\ \text { TO } & 39768\end{array}$

SOVENT Coc13

$\begin{array}{lc}05 & 0 \\ \text { 5.1 } & 6172.639 \mathrm{~Hz}\end{array}$

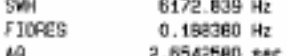

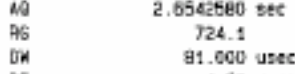

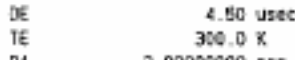

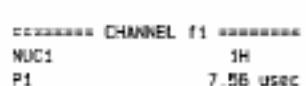

P1 7.56 usec

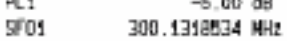

F2 - Processing psraneters

$\begin{array}{ll}\mathrm{SI} & 163 \mathrm{C} \\ \mathrm{FF} & 300.1350047 \mathrm{H}-\mathrm{z}\end{array}$

$\begin{array}{lr}55 & 300.1350047 \\ \text { Vow } & \text { EM } \\ 558 & 0\end{array}$

$\begin{array}{ll}\mathrm{LB} & 0.30 \mathrm{H} \\ \mathrm{SO} & 0\end{array}$

10 NAP plot paraneters
$c 2$
$22.00 \mathrm{c}$

$\begin{array}{ll}\mathrm{CX} & 22.00 \mathrm{~cm} \\ \mathrm{CY} & 15.00 \mathrm{ca}\end{array}$

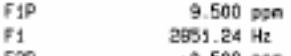

$7 \%-0.500$ pse

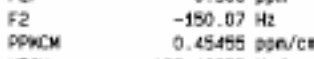

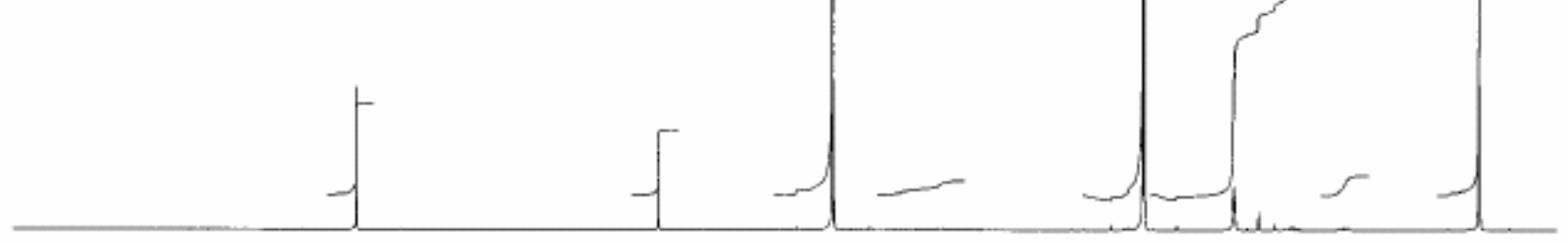

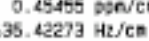

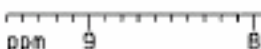

6

${ }_{5}^{1}$

4

$T_{3}$

zho-zb-103-p 


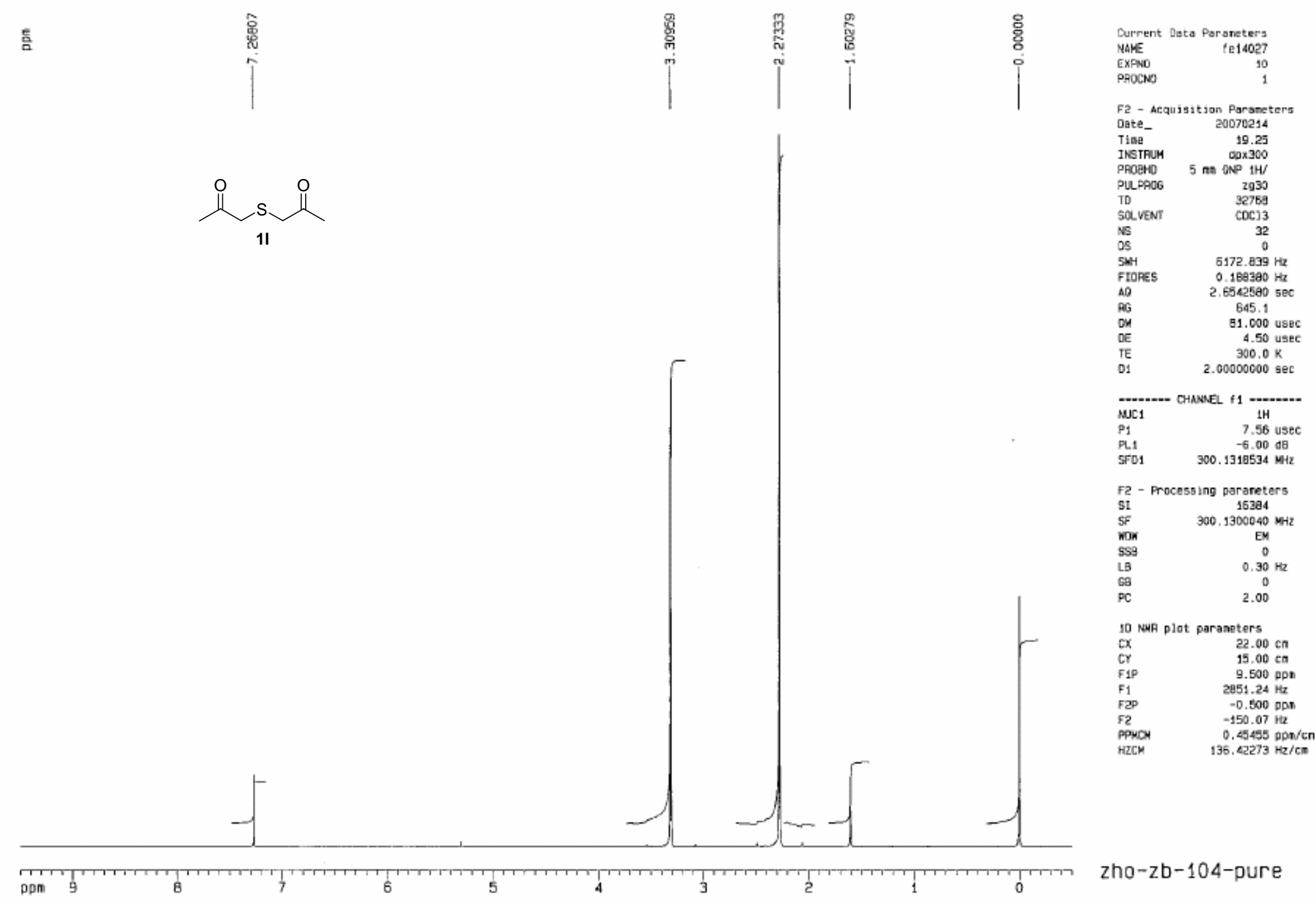



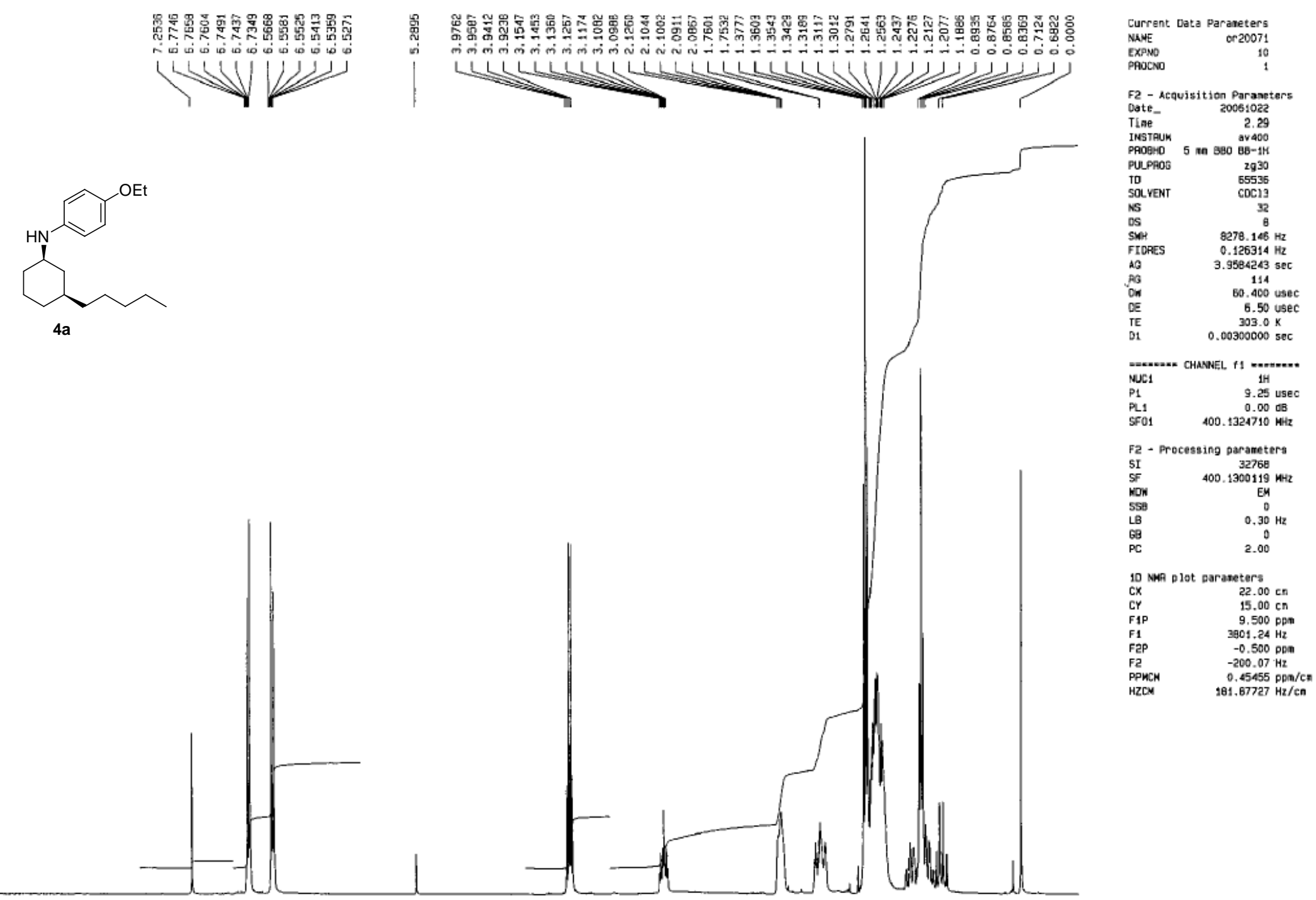

pn 9 8

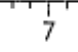

6

5 $T_{4}^{1}$ 3 2 1 


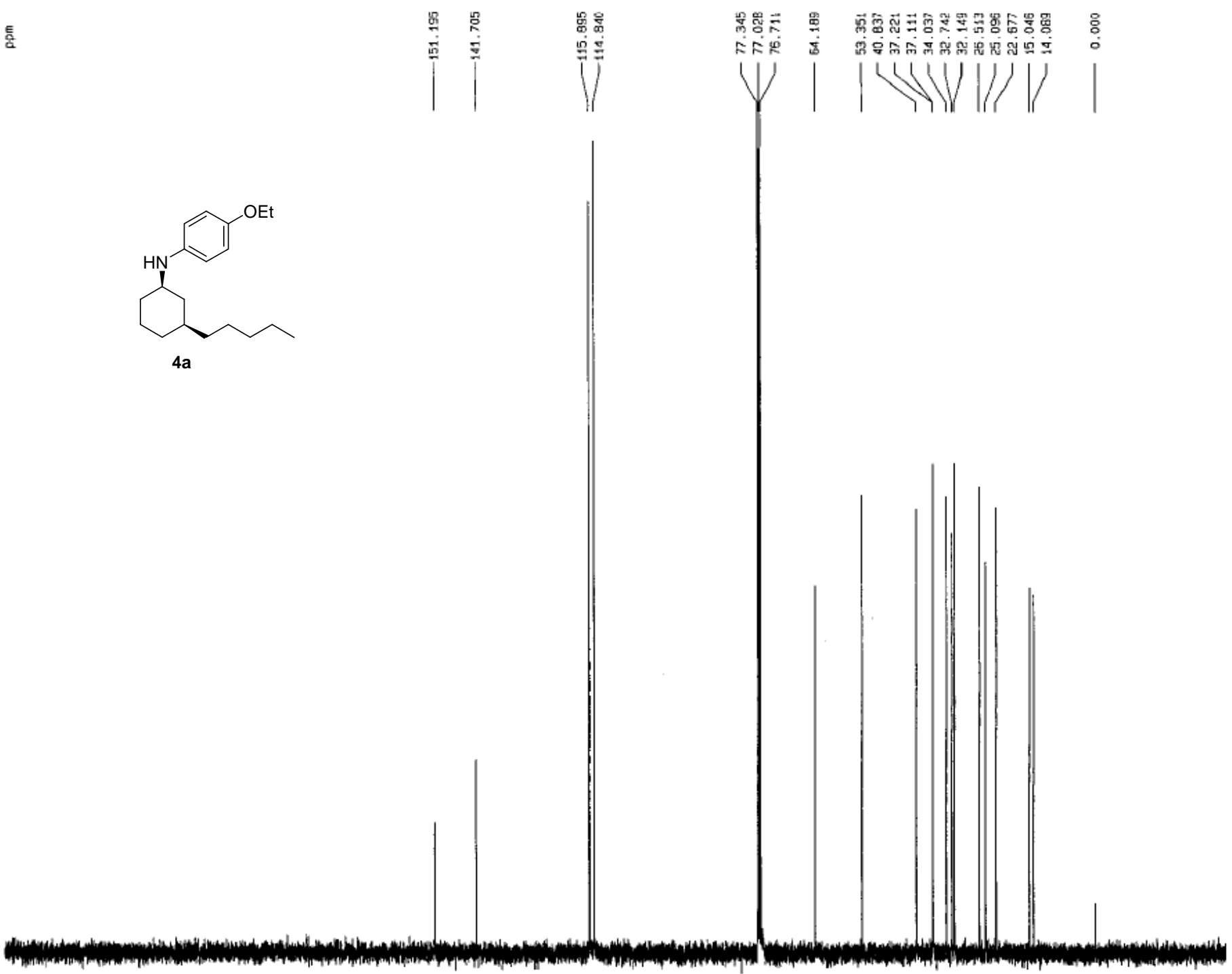

Current Data Paraneters

$\begin{array}{lr}\text { NAME } & \text { Or } 20071 \\ \text { DPPND } & 11 \\ \text { PFOCNO } & 1\end{array}$

Fe - Acquisition Par aneters

$\begin{array}{lr}\text { Date } & 20061022 \\ \text { Tine } & 2.59 \\ \text { INSTPIM } & \text { ave }\end{array}$

INSTPUM 5 m BBO $\mathrm{BV} 400$

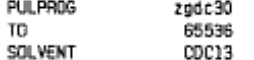

$\begin{array}{lr}\text { SOLVENT } & \text { COC13 } \\ \text { NS } & 1500 \\ \text { DS } & \end{array}$

$\begin{array}{lr}\text { SWH } & 33112.582 \mathrm{~Hz} \\ \text { FIDRES } & 0.505258 \mathrm{~Hz}\end{array}$

$\begin{array}{ll}\text { AO } & 0.505258 \mathrm{~Hz} \\ \text { AO } & 0.989436 \mathrm{sec}\end{array}$

$\begin{array}{ll} & 16354 \\ \text { ON } & 15.100 \text { usec } \\ \text { oe } & B .00 \text { usec }\end{array}$

$\begin{array}{ll}\text { TE } & 303.0 \mathrm{~K} \\ 01 & 0.0300000 \mathrm{sec}\end{array}$

......... CHawNEL $\mathrm{f1}$-........

NUCS
P1

$\begin{array}{ll}\text { PL1 } & 5.00 \mathrm{~dB} \\ \text { SF01 } & 100.5242789 \mathrm{HHz}\end{array}$

СР0PAG2 CHawNEL 12 -.......-.

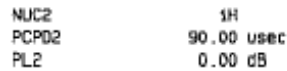

$\begin{array}{lr}\text { PL2 } & 0.00 \mathrm{~dB} \\ \text { PL12 } & 19.75 \mathrm{~dB} \\ \text { SF02 } & 400.1324710 \mathrm{kHz}\end{array}$

Fì - Processing paraneters

$\begin{array}{ll}\text { SI } & 32768 \\ \text { SF } & 100.6127653 \\ \text { NHz }\end{array}$

$\begin{array}{lc}\mathrm{NDN} & \mathrm{EH} \\ \text { SSB } & 0 \\ \mathrm{LB} & 1.00 \mathrm{~Hz} \\ \mathrm{Gg} & 0\end{array}$

PC 2.0

10 NMR plot paraneters
CX
$22.00 \mathrm{~cm}$

$\begin{array}{lr}\mathrm{CY} & \begin{array}{l}22.00 \mathrm{~cm} \\ \mathrm{~F}\end{array} \quad 250.000 \mathrm{~cm} \\ \mathrm{FPan}\end{array}$

$\begin{array}{ll}\text { F1 } & 25153.19 \mathrm{~Hz} \\ \text { Fap } & -30.000 \mathrm{pqg}\end{array}$

PPYCM $12.72727 \mathrm{pga} / \mathrm{c}$ 

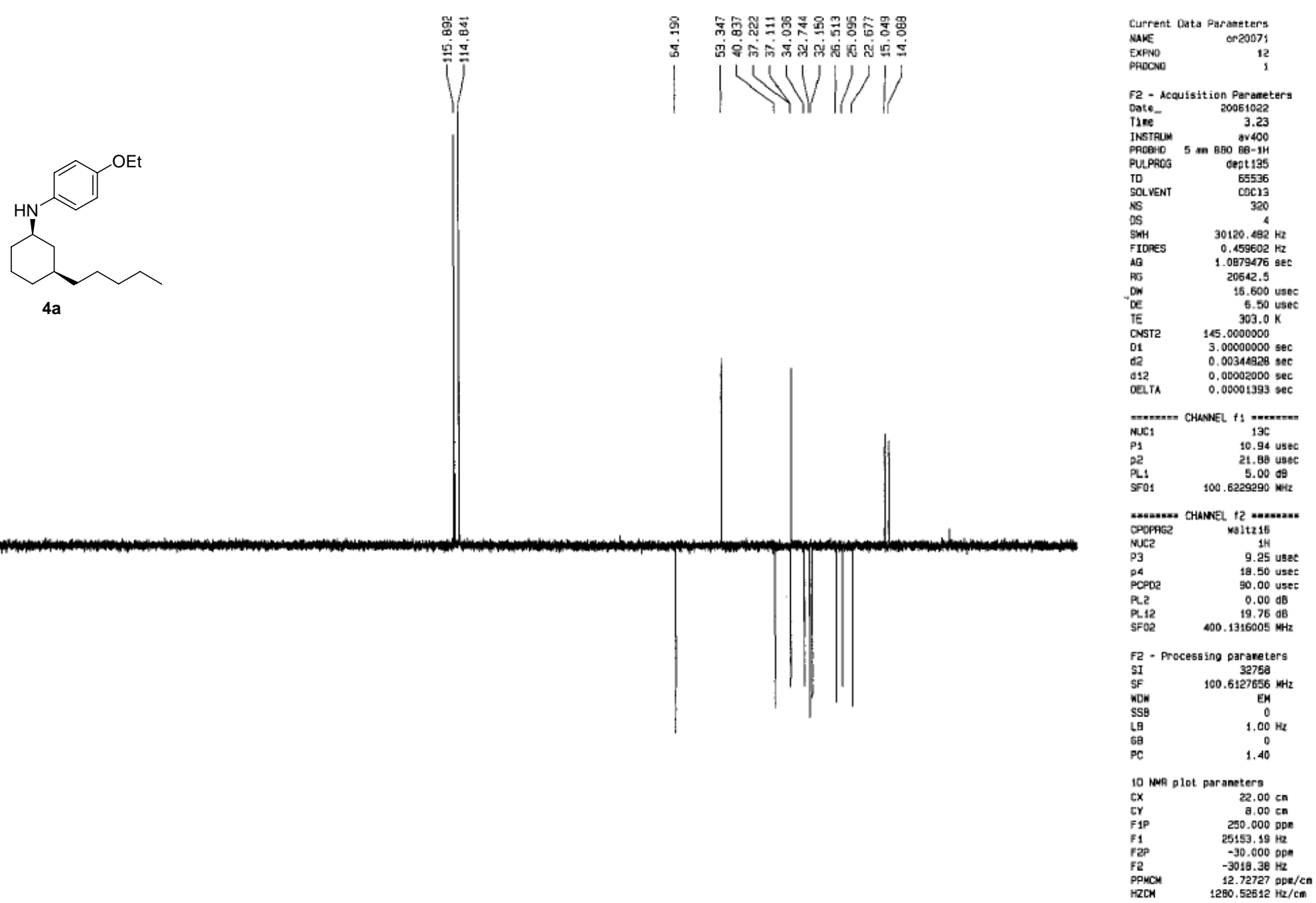

zho-za-480-cis 


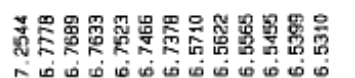

0

N

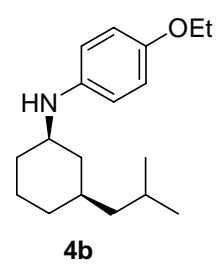

4b

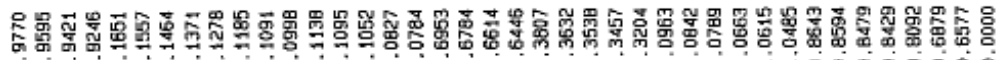

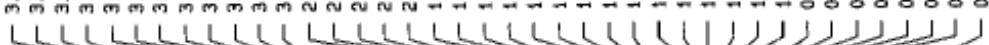
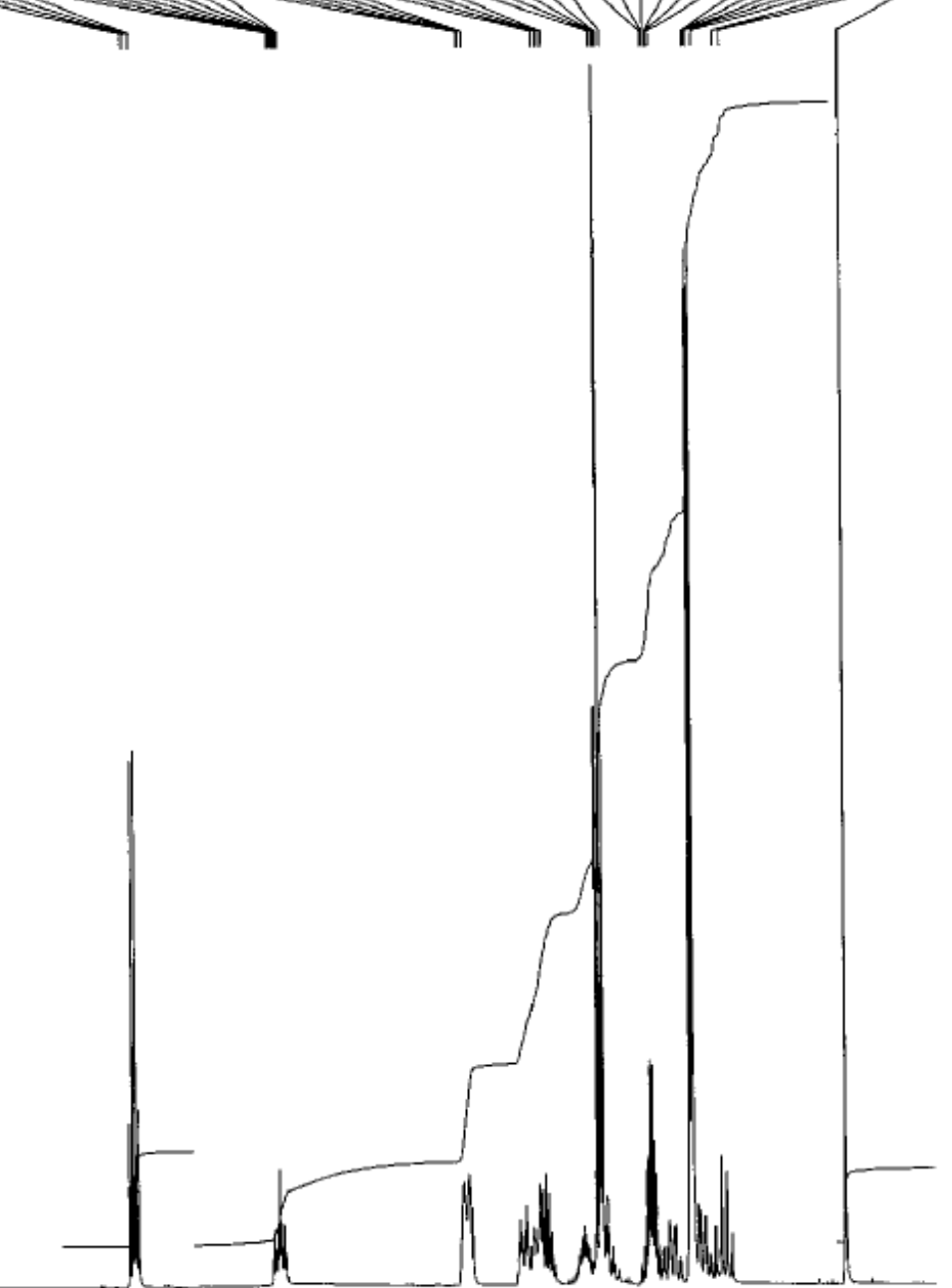

zho-za-402-cis
Current Dats Parameters
save
at24093

EXPNO

F2 - Acquisition Paraneters

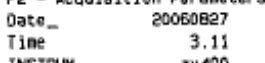

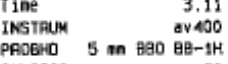

$\begin{array}{lr}2930 \\ \text { TD } & 65536 \\ \text { 5OLVENT } & \text { COC13 }\end{array}$

$\begin{array}{lr}\text { NS } & 32 \\ \text { OS } & \text { B }\end{array}$

$\begin{array}{ll}\text { SWH } & 8278.146 \mathrm{~Hz} \\ \text { EIDPES } & 0.126314 \mathrm{~Hz}\end{array}$

$\begin{array}{ll}A 0 & 3.9564243 \mathrm{sec} \\ \mathrm{AG} & 114\end{array}$

60.400 usec
OE
OE
TE
30.50 user

a1 $\quad \begin{aligned} & 303.0 \mathrm{~K} \\ & 0.0030000 \mathrm{sec}\end{aligned}$

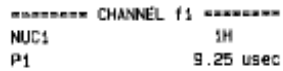

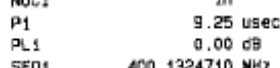

F2 - Processing paraneters

SI $\quad 300.1300119 \mathrm{MHz}$

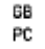

10 NMP plot paraneterg
CX
$22.00 \mathrm{~cm}$

$\begin{array}{ll}\mathrm{CX} & 22.00 \mathrm{~cm} \\ \mathrm{CY} & 15.00 \mathrm{~cm} \\ \mathrm{FIP} & 9.500 \mathrm{pPm}\end{array}$

$\begin{array}{ll}F 1 & 3801.24 \mathrm{~Hz} \\ F 2 P & -0.500 \mathrm{ppn}\end{array}$

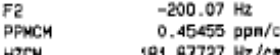

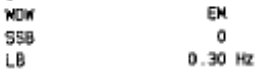

5

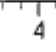

2

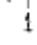

o ppm 9 

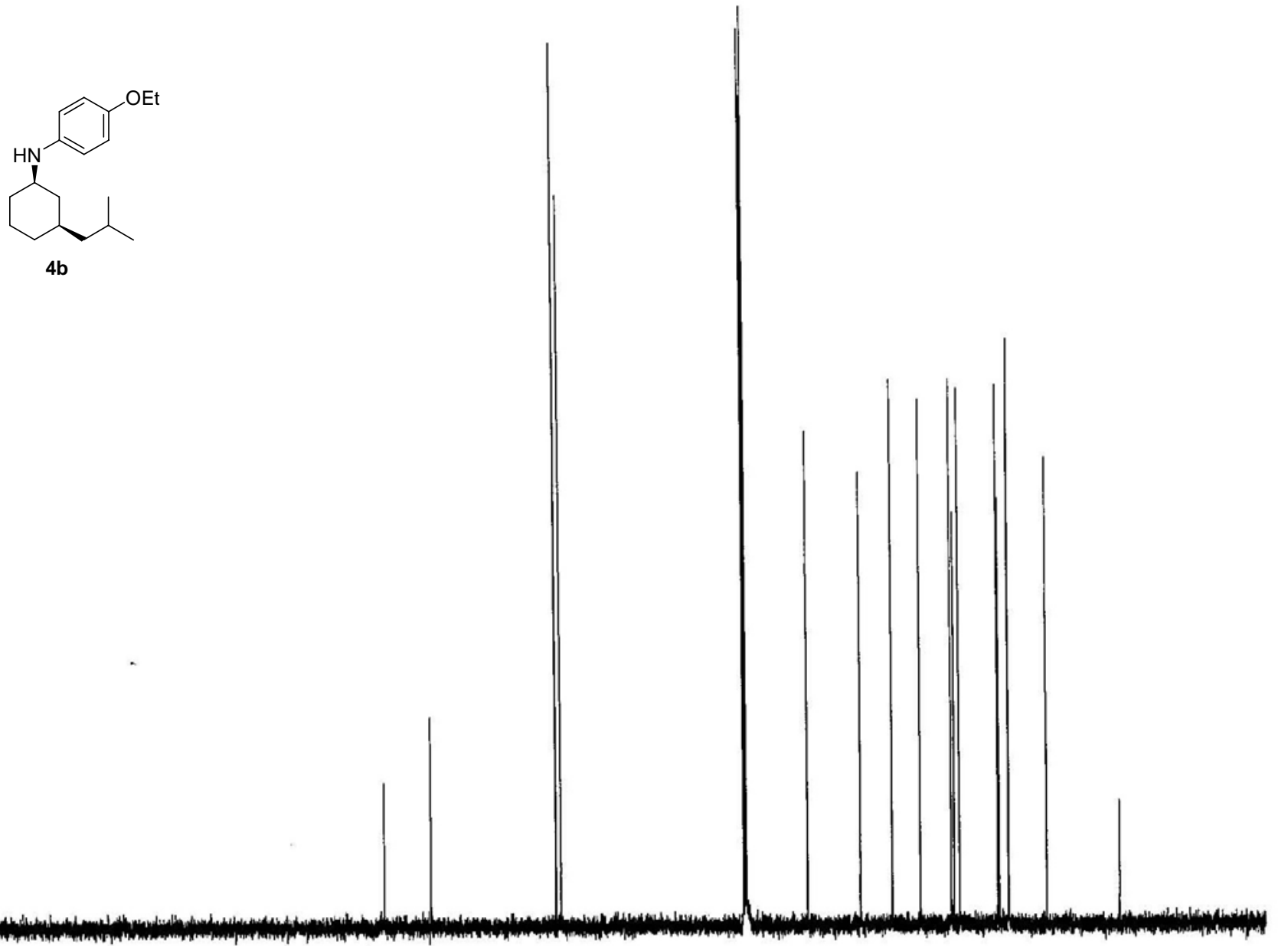

NAME at24093

PAOCNO

F2 - Acquigition Parameters

Date_ 20060027

$\begin{array}{lr}\text { Time } & 3.39 \\ \text { InSTIUM } & \text { av400 }\end{array}$

PAOEHO $5 \mathrm{~mm} B 80 \mathrm{BB}-\mathrm{SH}$
PULPROG
zgde 30

$\begin{array}{lr}\text { IO } & 65535 \\ \text { SOLVENT } & \text { COC13 } \\ \text { NS } & 1500\end{array}$

$\begin{array}{ll}\text { DS } & \text { 2 } \\ \text { SWH } & 33112.582 \mathrm{~Hz}\end{array}$

$0.505258 \mathrm{~Hz}$

$\begin{array}{ll}\text { AO } & 0.9996436 \mathrm{sec} \\ \text { AG } & 1690.4 \\ \text { OK } & 15.00 \mathrm{usec}\end{array}$

$\begin{array}{ll}\text { DE } & 8.00 \mathrm{usec} \\ \text { TE } & 303.0 \mathrm{~K}\end{array}$

$\begin{array}{ll}01 & 0.03000000 \mathrm{sec} \\ 011 & 0.03000000 \mathrm{sec}\end{array}$

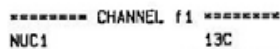

$\begin{array}{ll}\text { P1 } & 13.94 \mathrm{usec} \\ P_{1} & 5.00 \mathrm{dg}\end{array}$

$\begin{array}{lr}\text { PL1 } & 5.00 \mathrm{~dB} \\ \mathrm{SFO1} & 100.5242799\end{array}$

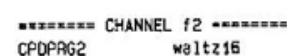

CPOPAG
NUC2

$\begin{array}{ll}\text { PCPO2 } & 90.00 \text { usec } \\ \text { PL2 } & 0.0000 \\ \text { PLe } & 19.76 \text { de }\end{array}$

PL.12
SF02 $\quad \begin{array}{ll}19.76 \\ \mathrm{de}\end{array}$

F2 - Processing parameters

$\begin{array}{ll}\text { SI } & \text { 32768 } \\ \text { SF } & 100.6127673\end{array}$

$\begin{array}{lc}\text { NON } & \text { EM } \\ \text { SSB } & 0 \\ \text { LB } & 1.00 \mathrm{HZ}\end{array}$

$\mathrm{GB}$
$\mathrm{PC}$

10 NHA plot parameters
CX $22.00 \mathrm{~cm}$

$\begin{array}{lr}\text { CY } & 15.00 \mathrm{~cm} \\ \text { F19 } & 250.000 \mathrm{pDg} \\ \text { F1 } & 25153.19 \mathrm{~Hz}\end{array}$

$\begin{array}{ll}F_{1} & 25153.19 \mathrm{~Hz} \\ \text { Fap } & -30.000 \mathrm{ppm}\end{array}$

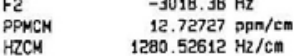



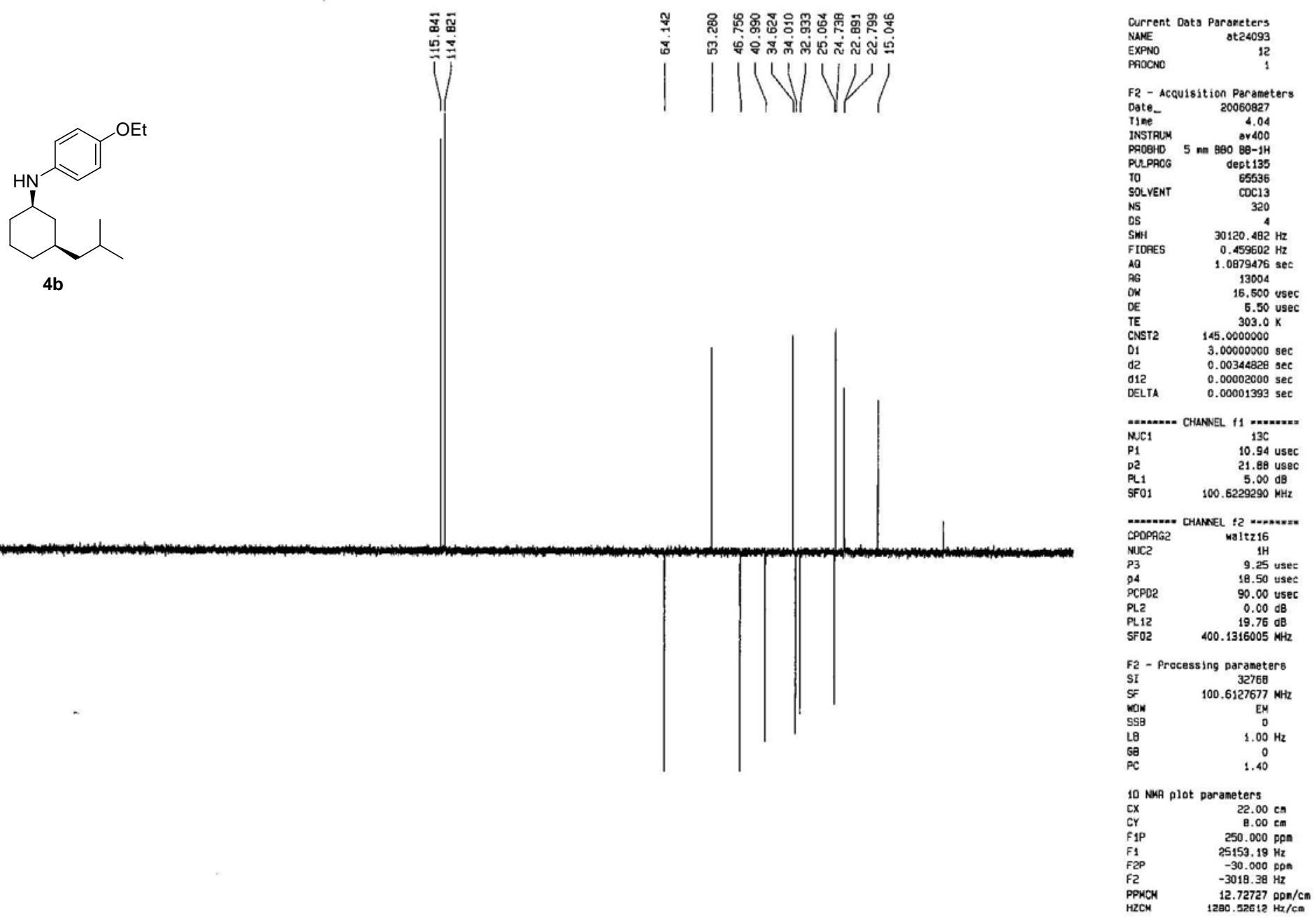
F2 - Acquisition Paraneters

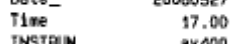

INSTRON
PROOHO

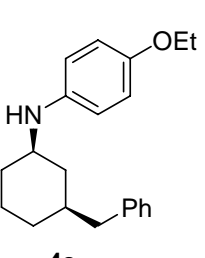

$\begin{array}{ll}\text { TD } & 65536 \\ \text { 50LVENT } & \text { CDCH13 }\end{array}$

$\begin{array}{lr}\mathrm{NS} & 32 \\ \mathrm{OS} & \mathrm{B}\end{array}$

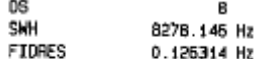

$\begin{array}{ll}{ }_{\text {AG }} & 0.126314 \mathrm{~Hz} \\ \text { AG } & 3.9564243 \\ \text { A6 } & 114\end{array}$

$\begin{array}{lr}\text { ON } & 60.400 \text { usec } \\ \text { DE } & 5.50 \text { ussec } \\ \text { TE } & 303.0 \mathrm{~K} \\ \text { DI } & 0.00300000\end{array}$

4c

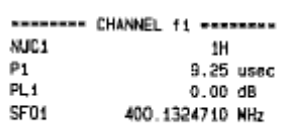

F2 - Processing paraneters

F2 - Processing paraneters
SI $\quad 32768$
SF
SO0.

$\begin{array}{lc}\text { NoN } & \text { SOM } \\ \text { SSB } & 0 \\ L B & 0.30 \mathrm{Kz}\end{array}$

$\begin{array}{ll}\mathrm{LB} & 0.00 \\ \mathrm{FC} & 2.00\end{array}$

10 NuF DJot paraneters

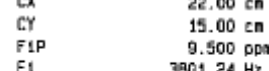

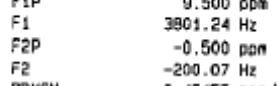

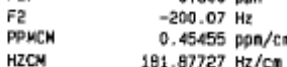

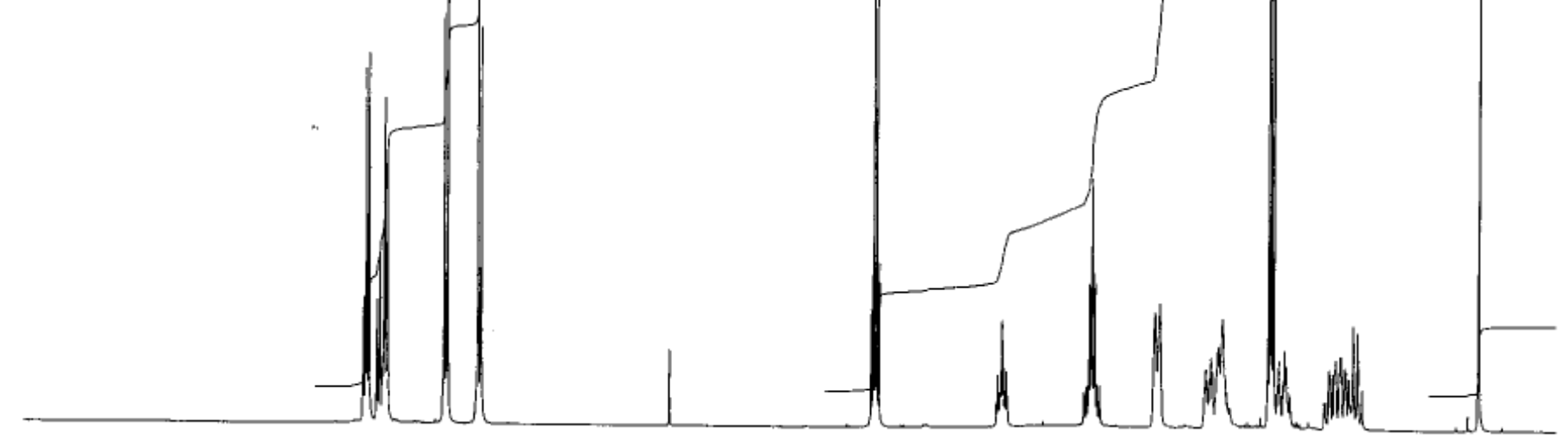

$87727 \mathrm{~Hz} / \mathrm{Cr}$

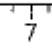

6

5 4

3

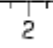

1 


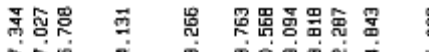

का

Vi ||||||

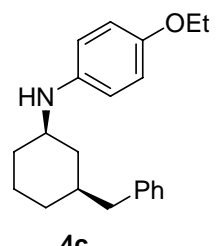

$4 c$

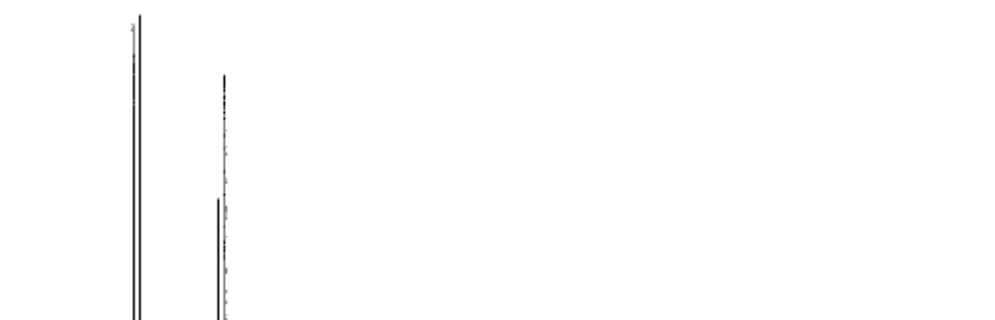

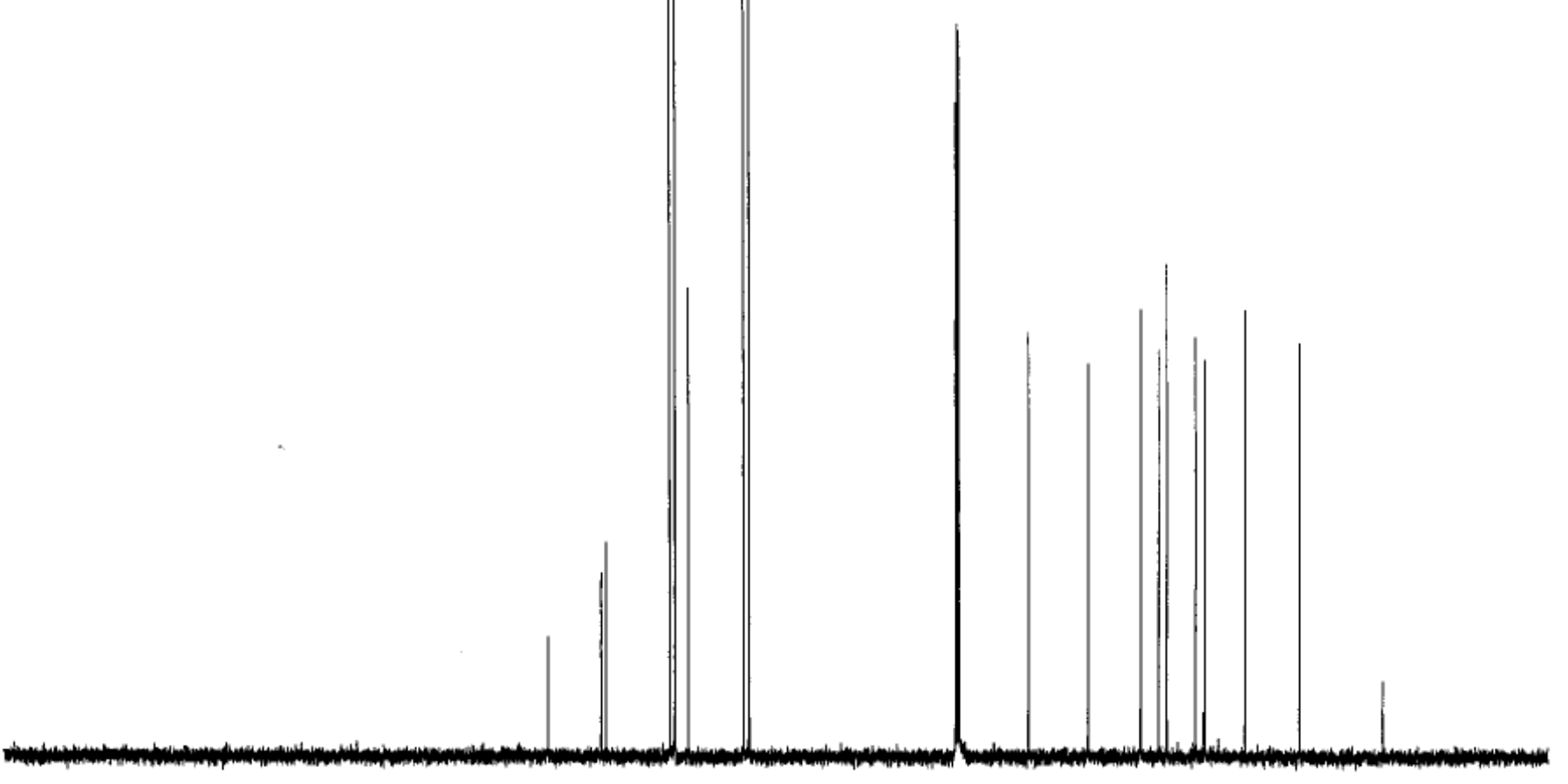



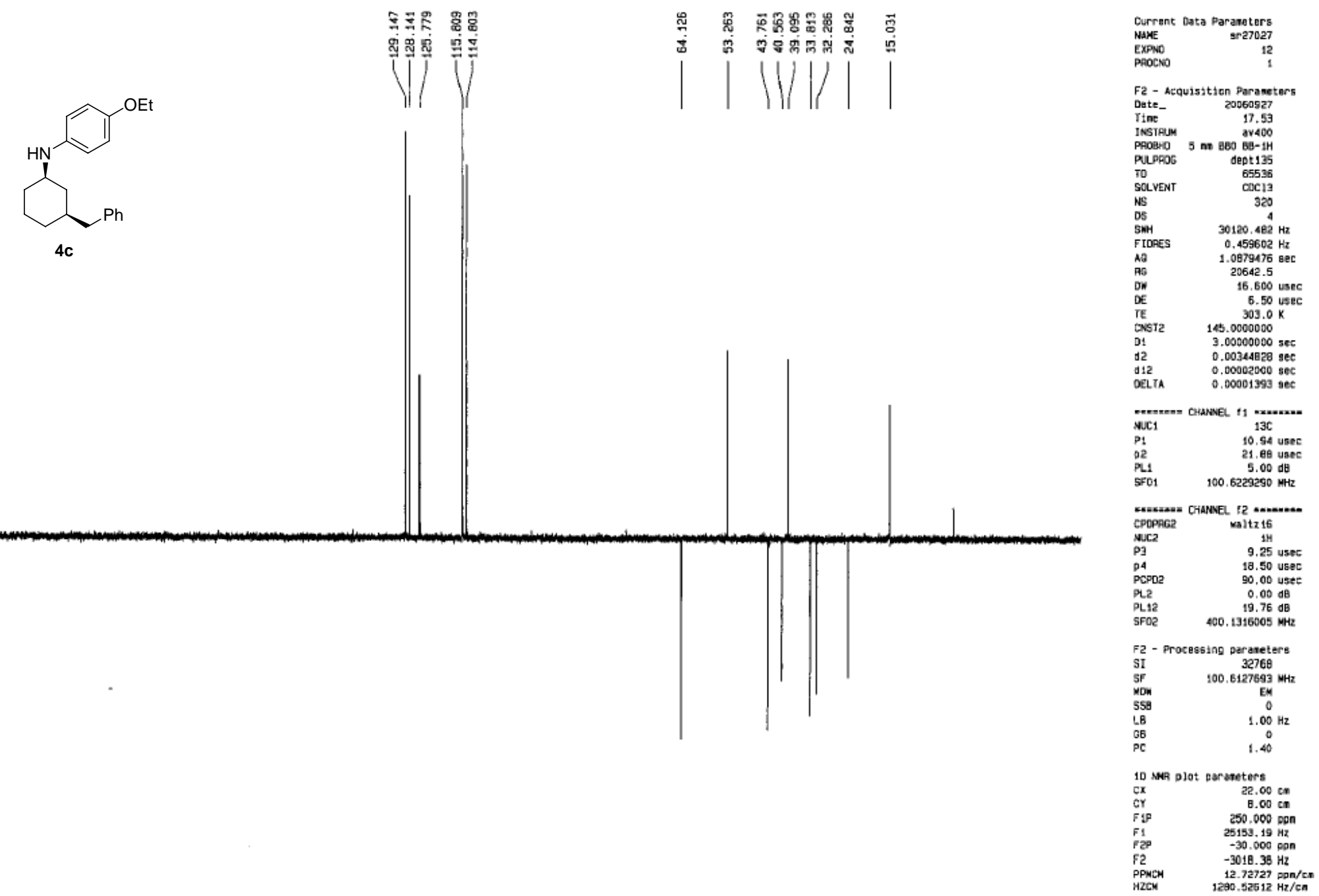

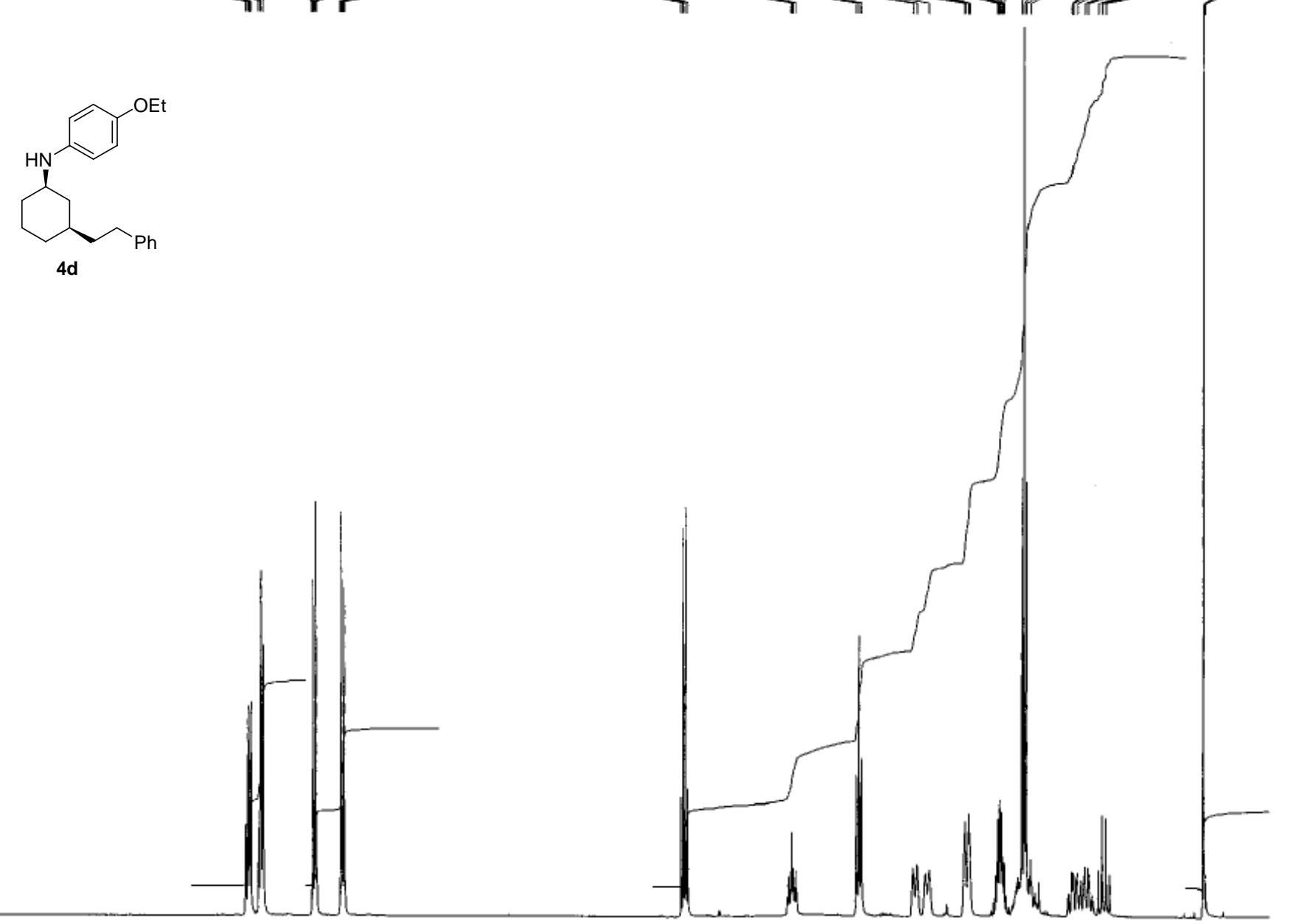

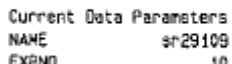

EXPNO
PAOCNO

Fe- Acquisition Paraneters

$\begin{array}{cc}\text { Fe. Acquisition Paranetern } \\ \text { Date_ } & 20051003 \\ \text { Tine } & 1.54\end{array}$

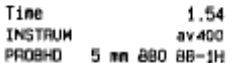

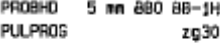

$\begin{array}{lr}\text { TD } & 65536 \\ \text { SOLVENT } & \text { CDC13 } \\ \text { NS } & 32\end{array}$

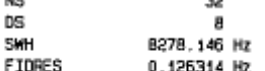

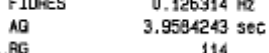

\begin{tabular}{ll} 
A AG & 114 \\
OW & 60.400 usec \\
OE & 6.50 usec \\
\hline
\end{tabular} D1 $\quad 0.00300000 \mathrm{sec}$

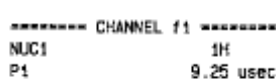

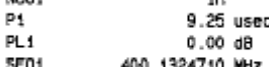

Fa - Processing paraneters

$\begin{array}{ll}\text { SI } & 32768 \\ \text { SF } & 000.1300156 \mathrm{WHz}\end{array}$

SS
48
$G B$
$\rho C$

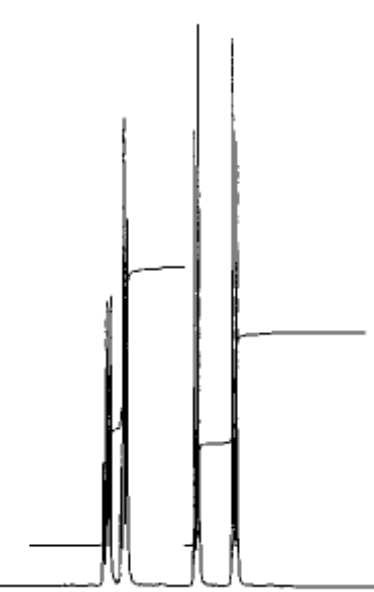

10 NMA Dlot paraneters

10 NWA Dlot paranaters
$C X$
$C Y$

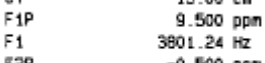

$\begin{array}{ll}\text { Fe } & -0.500 \\ \text { PPHCM } & -200.07 \mathrm{~Hz} \\ \text { PPH } & 0,45455 \mathrm{ppm} / \mathrm{cm}\end{array}$

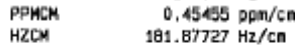




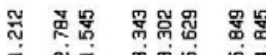

过

i

(1)

VI

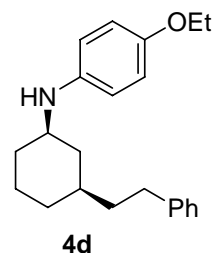

$4 d$
Current Data Parameters

NAME $\quad$ Sr29109

F2 - Acquisition Parameters

20061003
Time

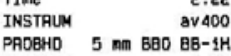

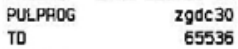

$\begin{array}{lll}\text { SOLVENT } & \text { COC19 } \\ \text { NS } & 1500\end{array}$

SWH $\quad 33112.58$ H $^{2} \mathrm{~Hz}$

FIDRES $\quad 0.505258 \mathrm{~Hz}$

$\begin{array}{ll}\text { AQ } & 0.98966356 \mathrm{sec} \\ \text { AG } & 16384\end{array}$

15.100 usec
8.00 usec

8.00 usec
$303.0 \mathrm{~K}$

$\begin{array}{ll} & 0.03000000 \mathrm{sec} \\ 01 & 0.03000000 \mathrm{sec}\end{array}$

NUC1 1 .... CHAWEL 11 .......

$\begin{array}{lr}\text { PLL } & 5.00 \mathrm{~dB} \\ \text { SF01 } & 100.5242789 \mathrm{HHz}\end{array}$

CPDPAG2 CHANNEL $\mathrm{f2}$.........

$\begin{array}{ll}\text { NUC2 } & 1 \mathrm{H} \\ \mathrm{PCPO2} & 90.00 \mathrm{usec}\end{array}$

PL2 $\quad 0.00 \mathrm{~dB}$

SFO2 $\quad 400.1324710 \mathrm{HHz}$

F2 - Processing parameters

$\begin{array}{ll}\text { SI } & 32768 \\ \text { SF } & 100.6127687 \\ \text { MOH } & \text { EM }\end{array}$

$\begin{array}{lc}\text { MCW } & \text { EM } \\ \text { SSB } & 0 \\ \text { L } & 100\end{array}$

$\begin{array}{ll}L B & 1.00 \\ \text { GB } & 0 \\ \text { PC } & 2.00\end{array}$

10 NWR plot parameters

$\begin{array}{rr}22.00 \mathrm{~cm} \\ \mathrm{CY} & 15.00 \mathrm{~cm} \\ 250.000 & \mathrm{om}\end{array}$

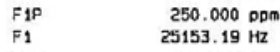

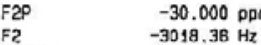

$\begin{array}{lr}P P M C H & 12.72727 \mathrm{pom} / \mathrm{cm} \\ \mathrm{HZCM} & 1280.52612 \mathrm{~Hz} / \mathrm{cm}\end{array}$ 


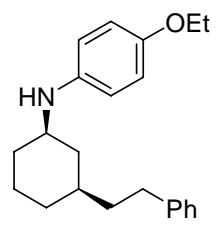

4d
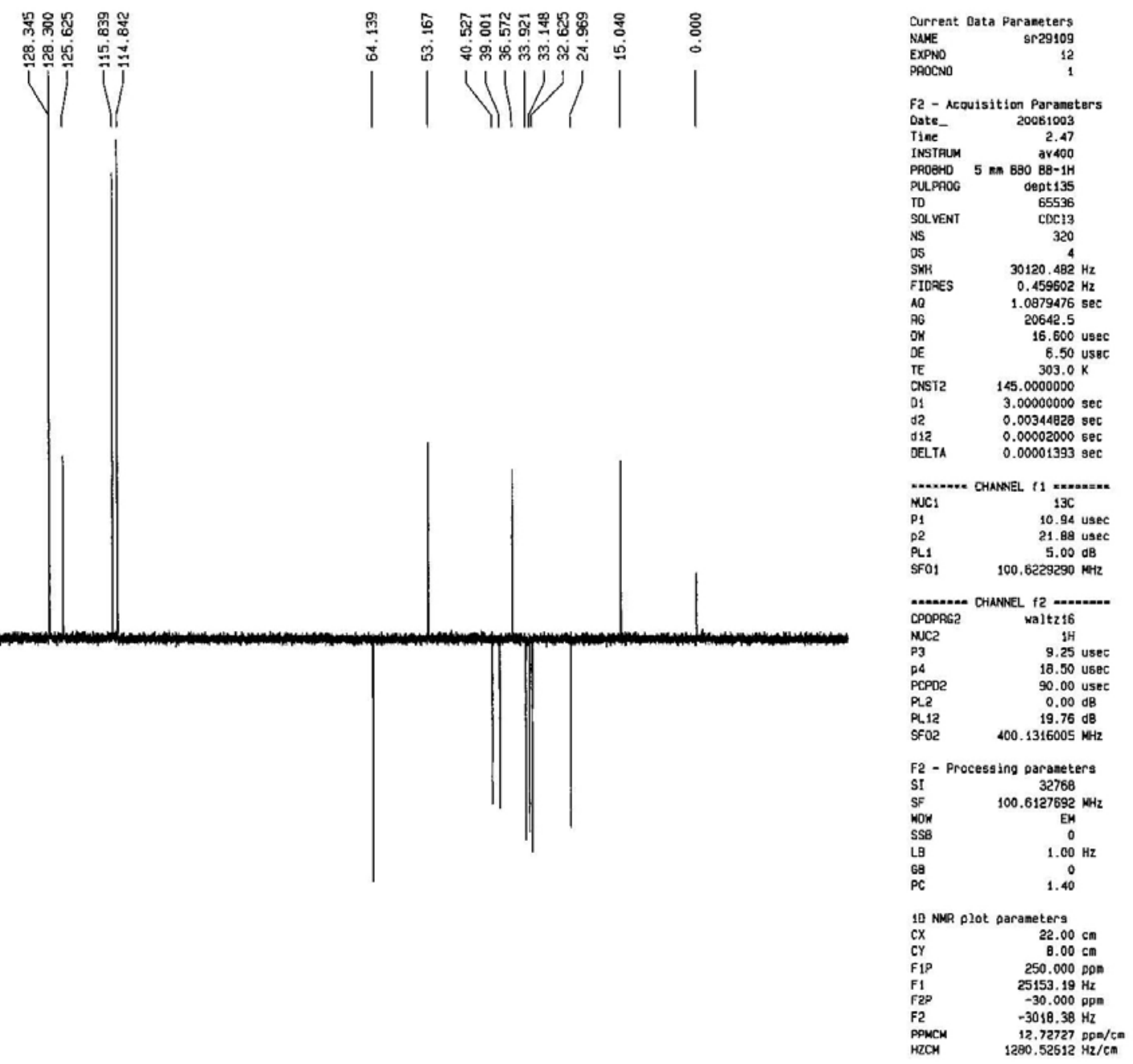

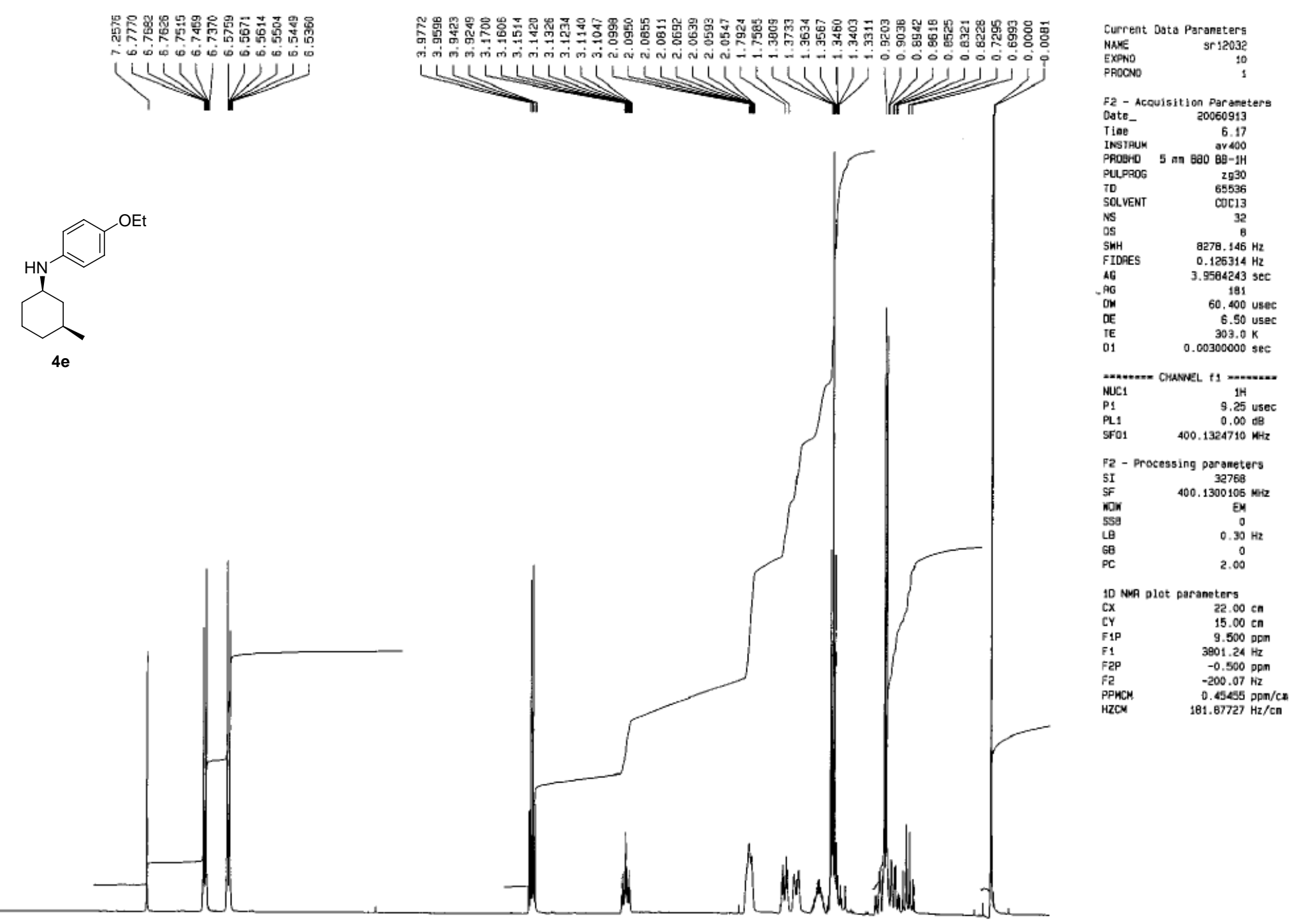

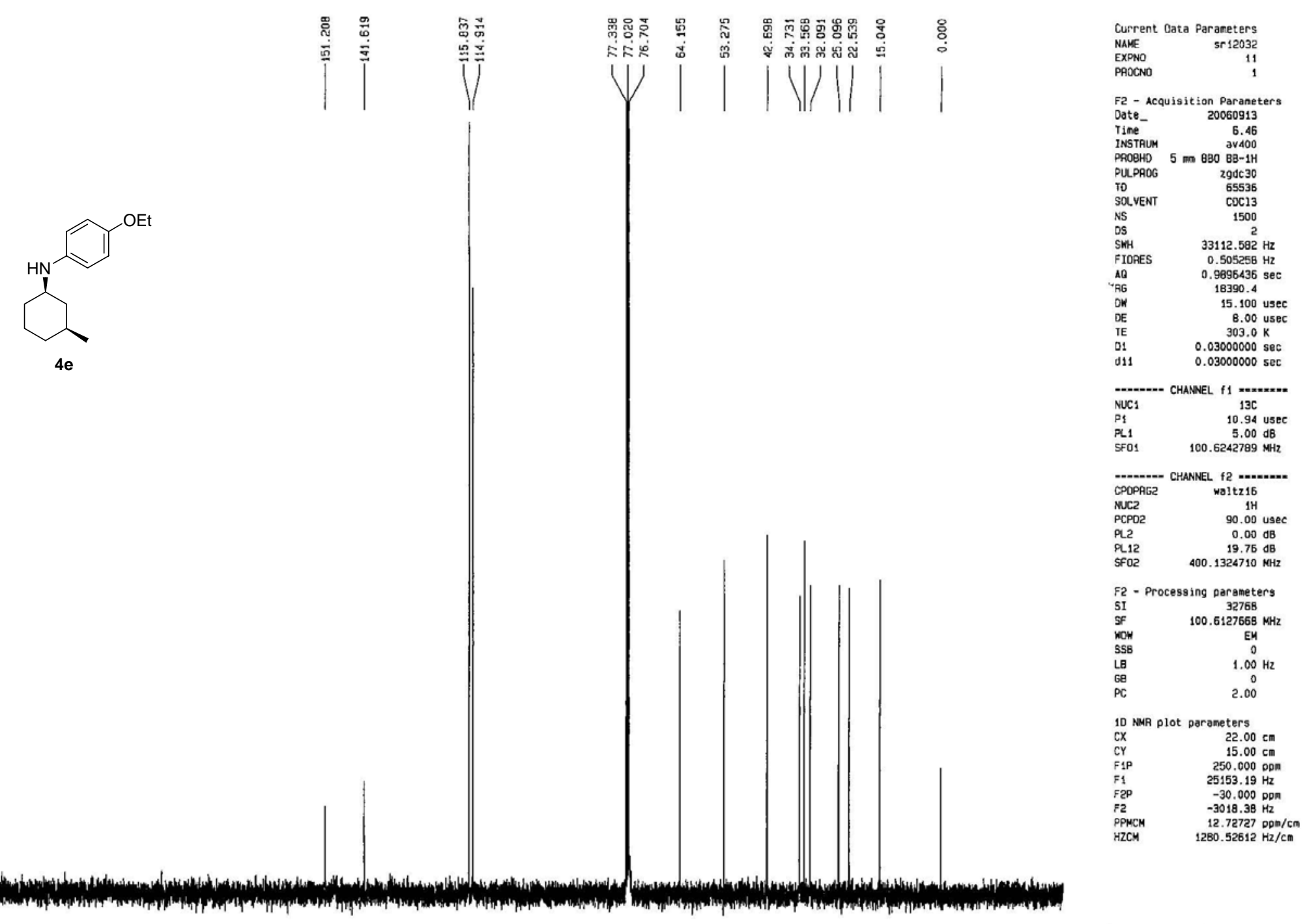


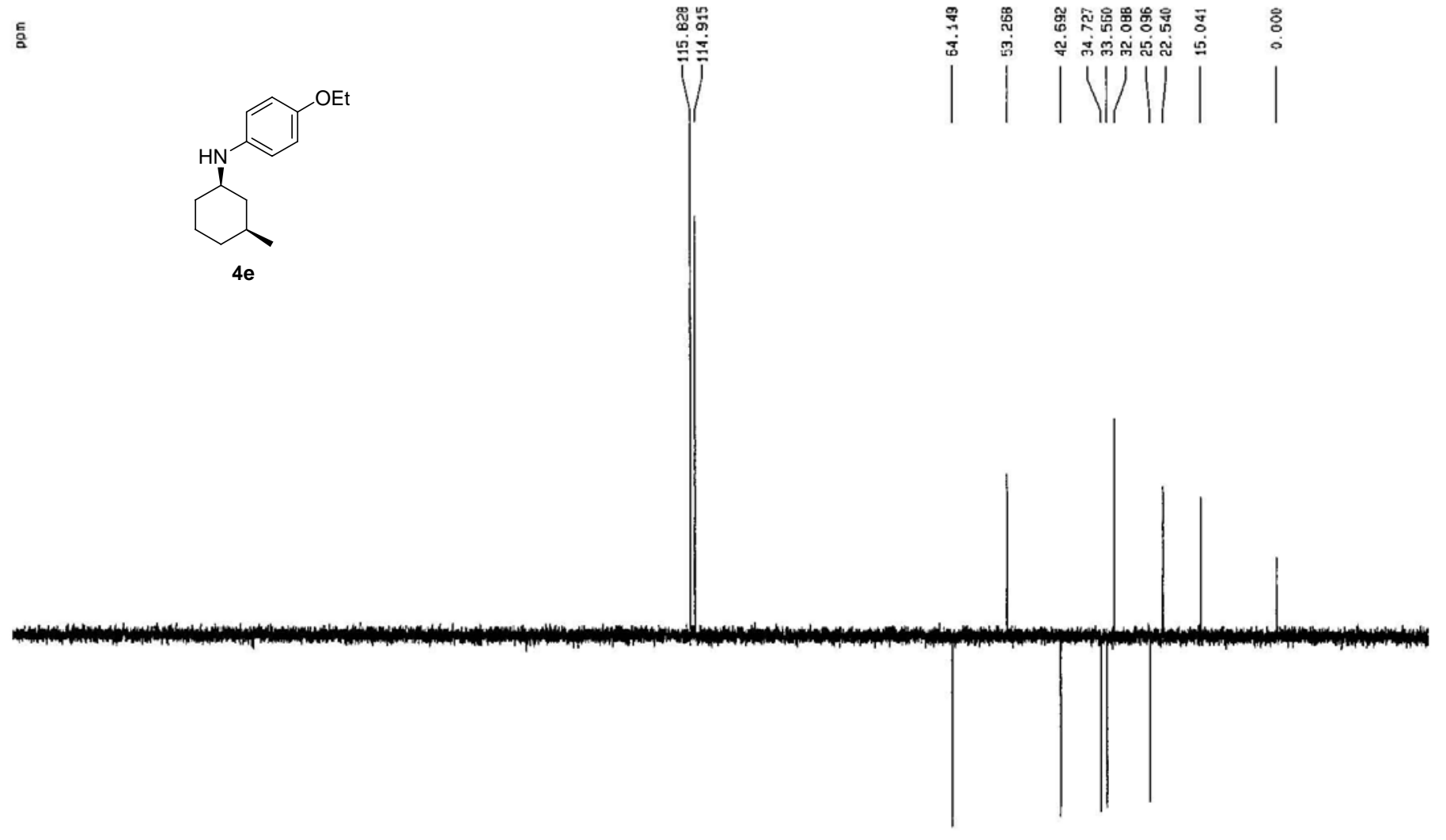

Current Data Parareters
vake

PXPOCOO

F2 - Acquisition Paraneters
20060913
Pate-

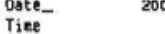

PULPPOG 5 em 800 80 $80-1400$

TD
SOLVENT
CDC13

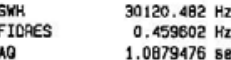

$\begin{array}{ll}A 0 & 1.0879476 \mathrm{sec} \\ \text { AG } & 20642.5 \\ \text { DW } & 16.600 \text { use } \\ \text { DE } & \end{array}$

$\begin{array}{lrl} & 6.50 \text { use } \\ \text { TE } & 303.0 \mathrm{~K}\end{array}$

$\begin{array}{ll}\text { CNST2 } & \begin{array}{l}145.0000000 \\ 01\end{array} \\ 0.00000000 & \mathrm{sec}\end{array}$

$\begin{array}{ll}0 & 0.00344828 \mathrm{sec} \\ 012 & 0.00002000 \mathrm{sec}\end{array}$

DELTA $0.00001393 \mathrm{sec}$

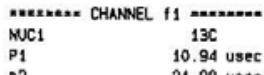

$\begin{array}{lr}P_{1} & 10.94 \text { usec } \\ \text { O2 } & 21.68 \text { usec } \\ \text { PL1 } & 5.00 \mathrm{de} \\ \text { SF01 } & 100.6229290 \mathrm{HHz}\end{array}$

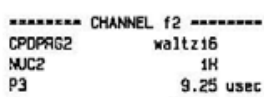

$\begin{array}{lr}9.25 \text { usec } \\ \text { PC } & 18.50 \text { usec } \\ \text { PCPO2 } & 90.0000\end{array}$

$\begin{array}{ll}\text { PCPO2 } & 90.00 \text { use } \\ \text { PL2 } & 0.00 \mathrm{~dB} \\ \text { P.12 } & 19.76 \mathrm{~dB}\end{array}$

SF02 400.1318005 det

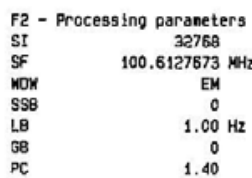

10 NMA plot paraneters
$\mathrm{CX}$
$\mathrm{CY}$

$\begin{array}{lr}C X & 22.00 \mathrm{~cm} \\ \mathrm{Cr} & 8.00 \mathrm{~cm} \\ \mathrm{FIP} & 250.000 \mathrm{~cm} \\ \mathrm{~F} 1 & 25153.19 \mathrm{~Hz}\end{array}$

$\begin{array}{ll}F 1 & 25153.19 \mathrm{~Hz} \\ \text { Frp } & -30.000 \mathrm{pon} \\ F 2 & -3018.39 \mathrm{~Hz}\end{array}$

PPHCM
HZCM
$1280.5272727 \mathrm{pDm} / \mathrm{cm}$

ppm

200

150

100

50

0

zho-za-400b-cis 

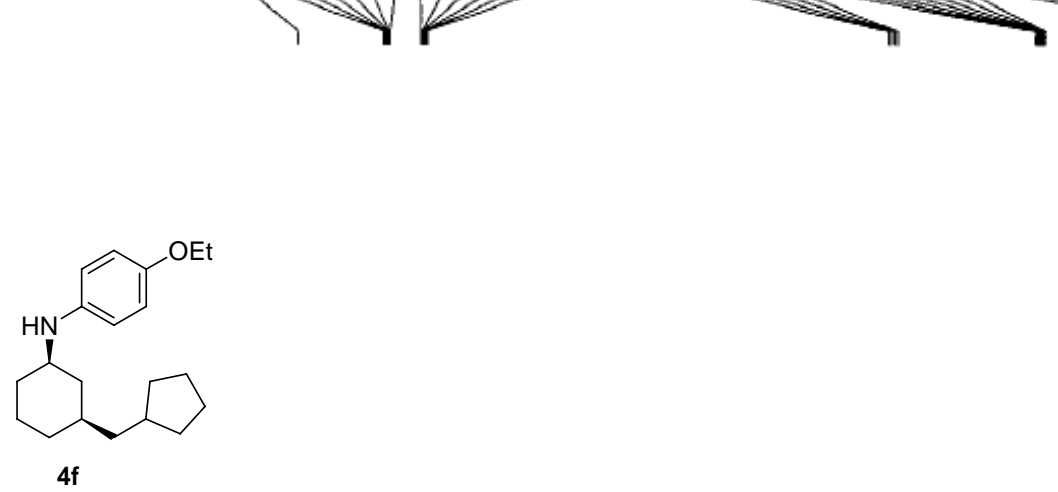

4

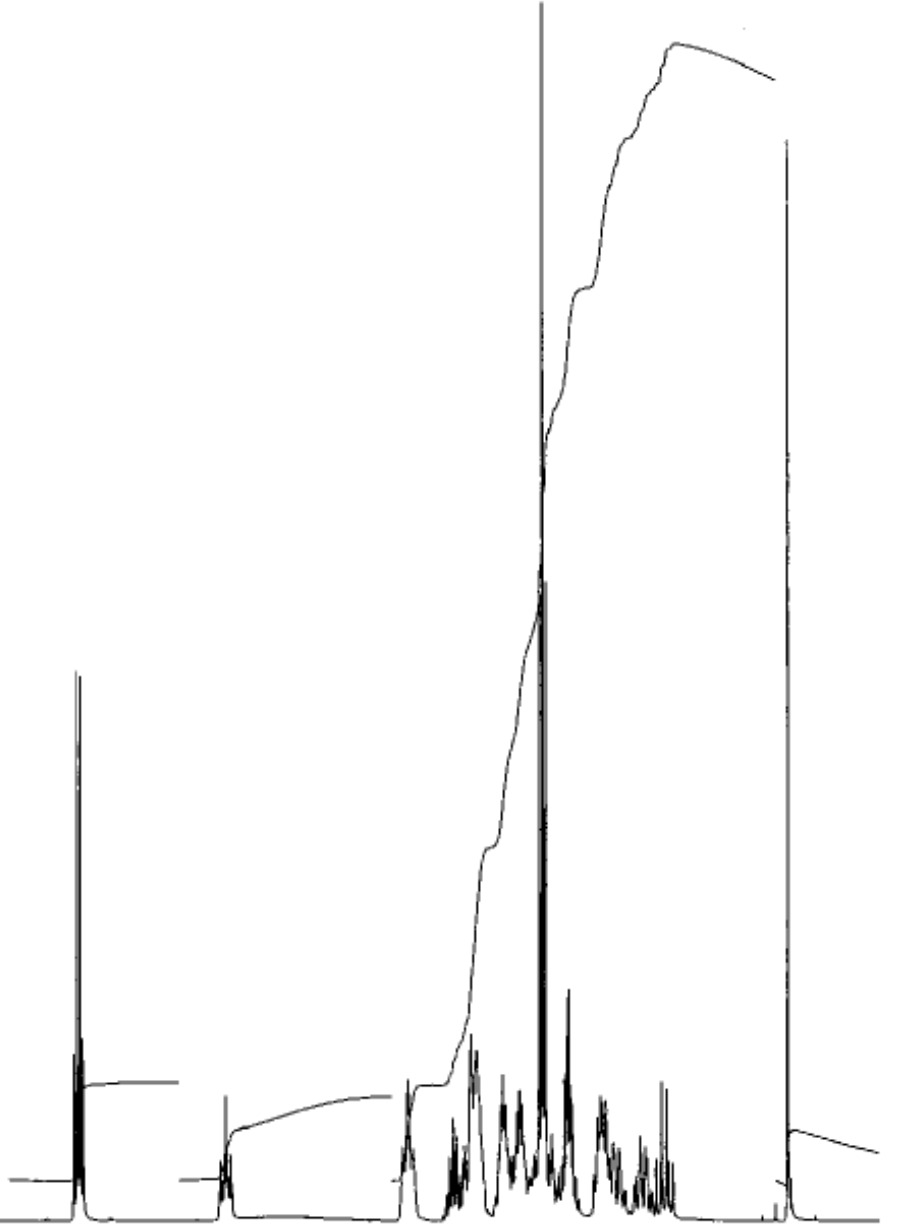

Current Data Pargneters
NAAE
EXPNO
PHOCNO

F2 - Acquisition Paraneters
Dote- 20060930

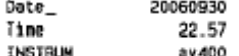

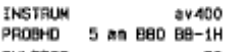

$\begin{array}{lr}2930 \\ \text { PULPADG } & 65536 \\ \text { TD } & \text { C0C13 }\end{array}$

$\begin{array}{lr}\text { SOLVENT } & \mathrm{COC13} \\ \text { NS } & 32 \\ \text { OS } & \text { a }\end{array}$

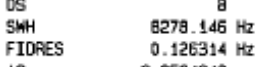

$\begin{array}{ll}A B & 3.9584243 \mathrm{sec} \\ \mathrm{AG} & 80.6\end{array}$

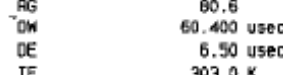

$0.103 .0 \mathrm{~K}$
$0.0030000 \mathrm{sec}$

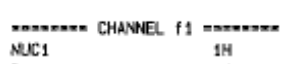

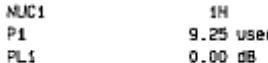

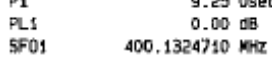

Fe - Processing paraneters

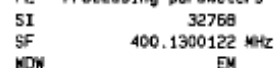

$\begin{array}{lc}\text { NON } & \text { EM } \\ 558 & 0 \\ \text { LB } & 0.30 \mathrm{~Hz} \\ 6 B & 0\end{array}$

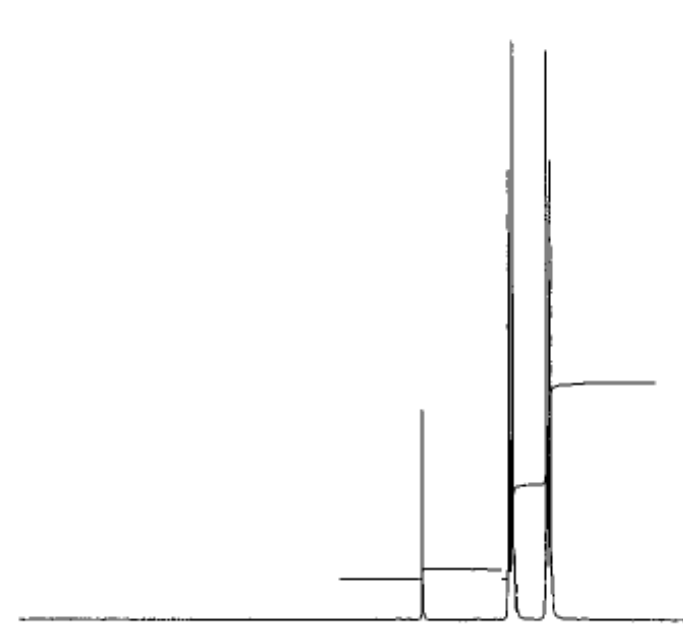

10 NiH plat paraneters
22,00

$\begin{array}{ll}\mathrm{CX} & 22,00 \mathrm{~cm} \\ \mathrm{Cr} & 15,00 \mathrm{~cm} \\ \mathrm{Fin} & 5050 \mathrm{man}\end{array}$

$\begin{array}{ll}F 1 P & 9.500 \mathrm{pen} \\ \mathrm{F} 1 & 3801.24 \mathrm{~Hz}\end{array}$

$\begin{array}{ll}\text { F2P } & -0.500 \mathrm{pPm} \\ \mathrm{FP} & -200.07 \mathrm{~Hz}\end{array}$

$\mathrm{PPHCM}$
$\mathrm{HZCN}$
$181.857727 \mathrm{HZ} / \mathrm{Cm}$
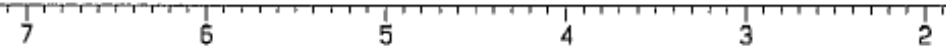

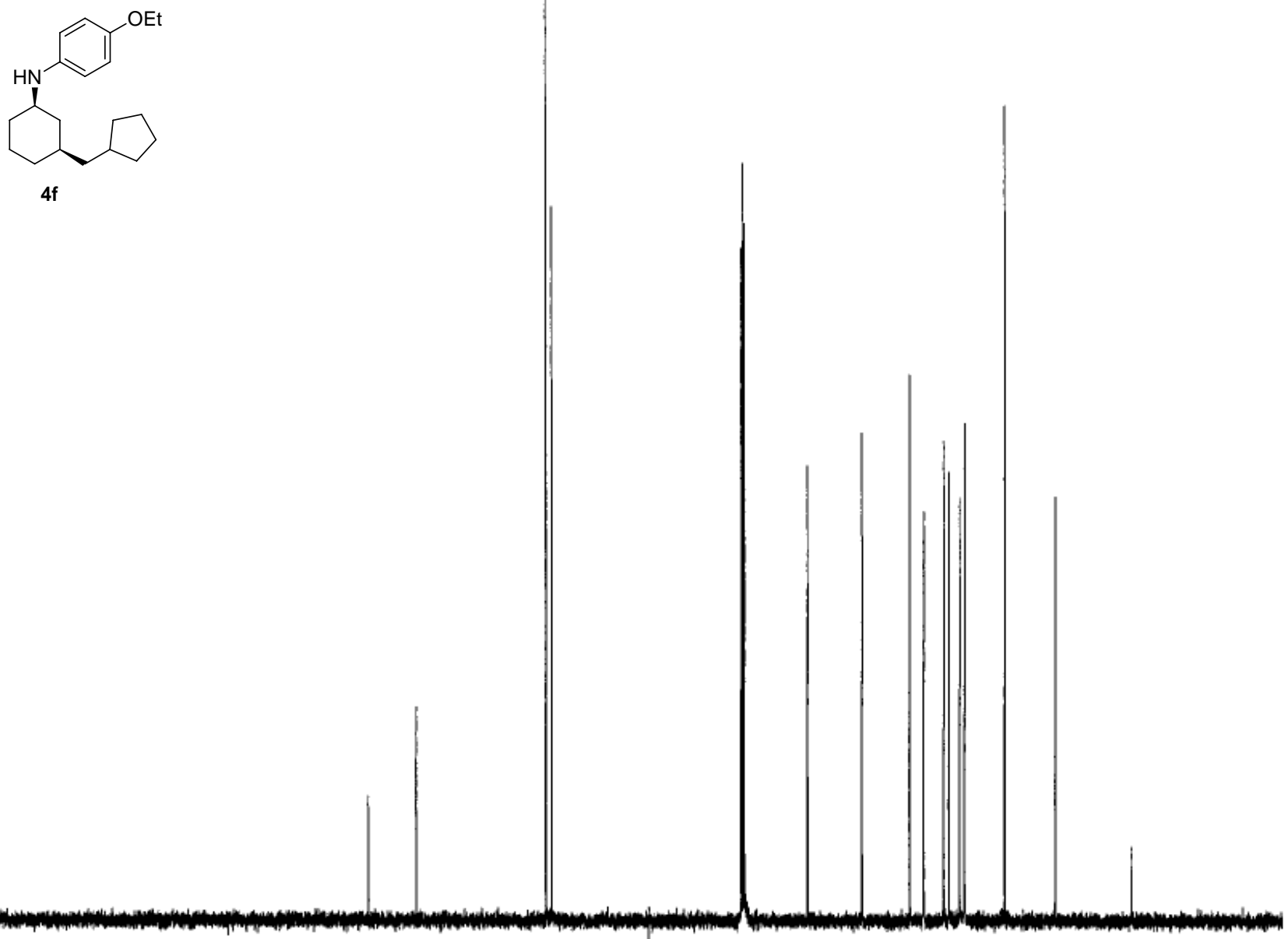

Current gata Paraneters

$\begin{array}{lr}\text { EXPNO } & \text { ST2906 } \\ \text { PFOCNO } & 11\end{array}$

Fa - Acquisition Paranaters

$\begin{array}{lr}\text { Date- } & 20060930 \\ \text { Tine } & 23.25 \\ \text { INSTAum } & \text { av } 400\end{array}$

INSTAUM
PFOBHO 5 m 830 BVAO-1H

PULPAOG
TD
TDdc 30
55536

$\begin{array}{lr}\text { SOLVENT } & \text { COC13 } \\ \text { NS } & 1500 \\ \text { OS } & \end{array}$

$33112.552 \mathrm{~Hz}$
SWH
FTOAES
$0.505258 \mathrm{~Hz}$

$\begin{array}{ll}\text { FIOAES } & 0.505258 \mathrm{~Hz} \\ \text { AD } & 0.9896436 \mathrm{sec}\end{array}$

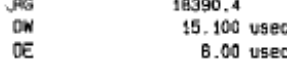

TE $303.0 \mathrm{~K}$

$\begin{array}{ll}\text { dit } & 0.03000000 \mathrm{sec} \\ \text { dis } & 0.03000000 \mathrm{sec}\end{array}$

MCI..... CHANNEL F1 =........

$\begin{array}{ll}\text { MUC1 } & 13 \mathrm{C} \\ \mathrm{P}_{1} & 10.94 \text { usec }\end{array}$

$\begin{array}{lr}P_{1} & 10.94 \\ \text { PL1 } & 5.00 \text { uge } \\ \text { SFO1 } & 100.6242799 \mathrm{HHz}\end{array}$

CPDPPG CHANNEL I2 ................

$\begin{array}{ll}\text { NDC2 } & \text { SH } \\ \text { PCPOS } & 90.00 \text { usec }\end{array}$

$\begin{array}{lr}\text { PL2 } & 0.00 \mathrm{~dB} \\ \text { PL12 } & 19.76 \mathrm{~dB} \\ \text { SFro2 } & 400.1324710 \mathrm{HHz}\end{array}$

F2 - Processing paraneters

$\begin{array}{ll}\text { SI } & 32768 \\ \text { SF } & 100.6127675 \\ \mathrm{HHZ}\end{array}$

NOW

$\begin{array}{ll}\mathrm{LB} & 1.00 \mathrm{~Hz} \\ \mathrm{CB} & 0 \\ \mathrm{PC} & 2.00\end{array}$

to NWF plot paraneterg

$\begin{array}{lr}C X & 22.00 \mathrm{~cm} \\ \mathrm{CY} & 15.00 \mathrm{co} \\ \mathrm{CY} & 250.000 \mathrm{00m}\end{array}$

Fip $\quad 250.000 \mathrm{pom}$
$F_{1}$
$25153.19 \mathrm{~Hz}$

$\begin{array}{ll}\mathrm{F}^{2 P} & -30.000 \mathrm{ppm} \\ \mathrm{FP} & -3018.3 \mathrm{~Hz}\end{array}$

$\begin{array}{lr}\text { PPHCM } & 12.72727 \mathrm{gpm} / \mathrm{cm} \\ \mathrm{HZCM} & 1280.52612 \mathrm{~Hz} / \mathrm{cm}\end{array}$

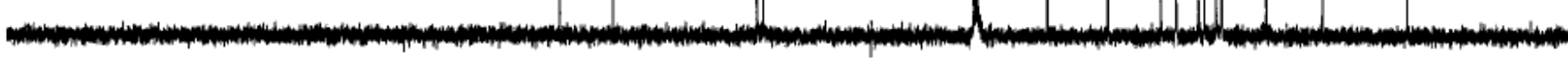

zho-za-474-cis 


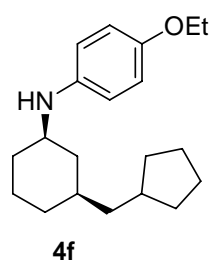

Current data Paraneters
NAME
EXPND
ERr2906

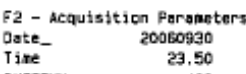

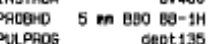

To
Solvent

Ns
os
os

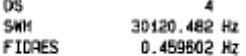

40

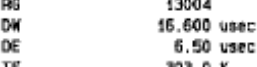

Cost2 145.0000000

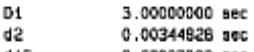

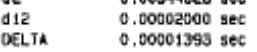

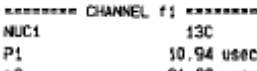

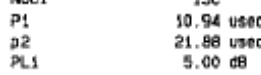

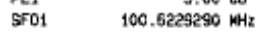

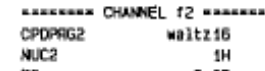

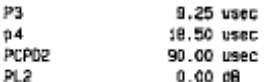

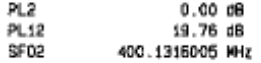

F2 - Processing paraneters

sI
SI
SP

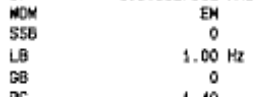

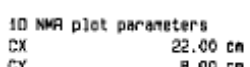

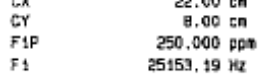

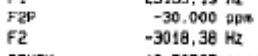

Perlow
PreC 


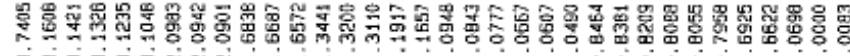

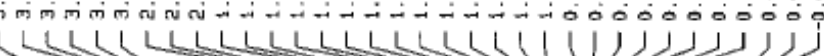

Curront Data Paraneters

$1+1$

L L L L L L L L L L L

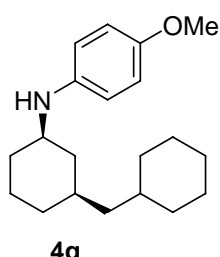

$4 \mathrm{~g}$
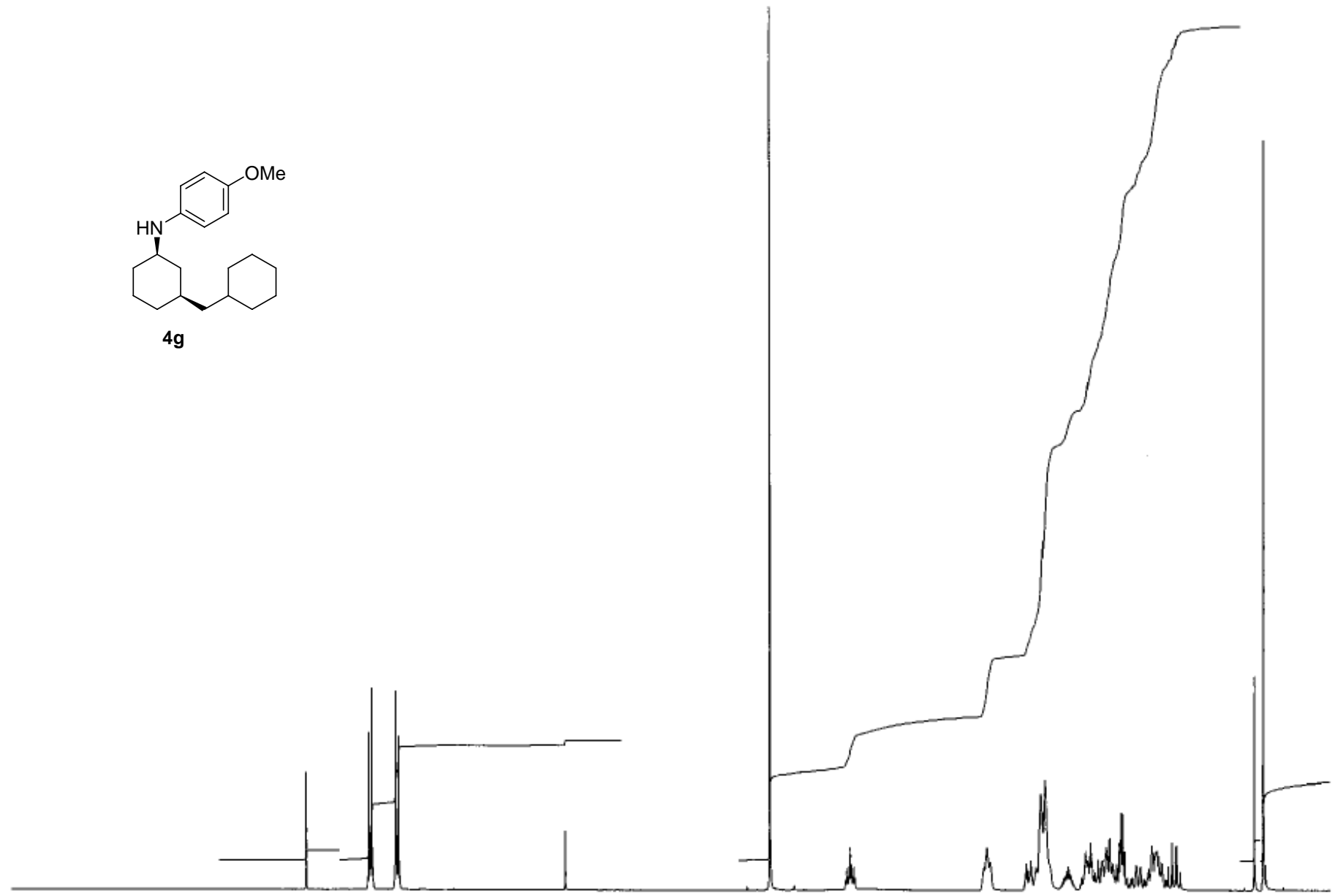

F2. - Acquisition Parareters
Date- 20050829

Date-

Tine
INSTAUM
16.06

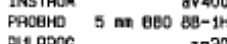

$\begin{array}{lr}\text { TD } & 59536 \\ \text { SOLVENT } & 55513 \\ \text { NS } & 32\end{array}$

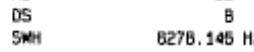

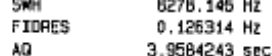

PO

60.400 unece
8.50 unec
DE

$\begin{array}{ll}\text { TE } & \begin{array}{c}303.0 \mathrm{~K} \\ 01\end{array} \\ 0.00300000 \mathrm{sec}\end{array}$

NuCS
$\mathrm{P}_{1}$

$\begin{array}{lr}\text { P1 } & 9.25 \text { usec } \\ \text { PL1 } & 0.00 \text { ob } \\ \text { SF-01 } & 00.1324710 \mathrm{MHz}\end{array}$

F2 - Processing paraneters

SI $\quad \begin{array}{ll}\text { SI } & 32768 \\ \text { SF } & 400.1300113 \\ \text { NHHZ }\end{array}$

$\begin{array}{cc}\mathrm{NON} & \mathrm{EM} \\ \mathrm{SSB} & 0 \\ \mathrm{LBB} & 0.30 \mathrm{~Hz} \\ \mathrm{~GB} & 0\end{array}$

10 NAP piot paranetors

10
$\mathrm{CX}$
$\mathrm{CX}$

$\begin{array}{ll}\mathrm{CY} & 15.00 \mathrm{ch} \\ \mathrm{F} 1 \mathrm{P} & 9.500 \mathrm{ppm}\end{array}$

$\begin{array}{ll}F 1 & 3601.24 \\ \text { F } & -0.52\end{array}$

$\begin{array}{ll}\mathrm{FP} & -200.07 \mathrm{~Hz} \\ \mathrm{PPQCH} & 0.45455 \mathrm{pPm} / \mathrm{cm}\end{array}$

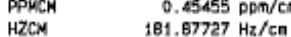



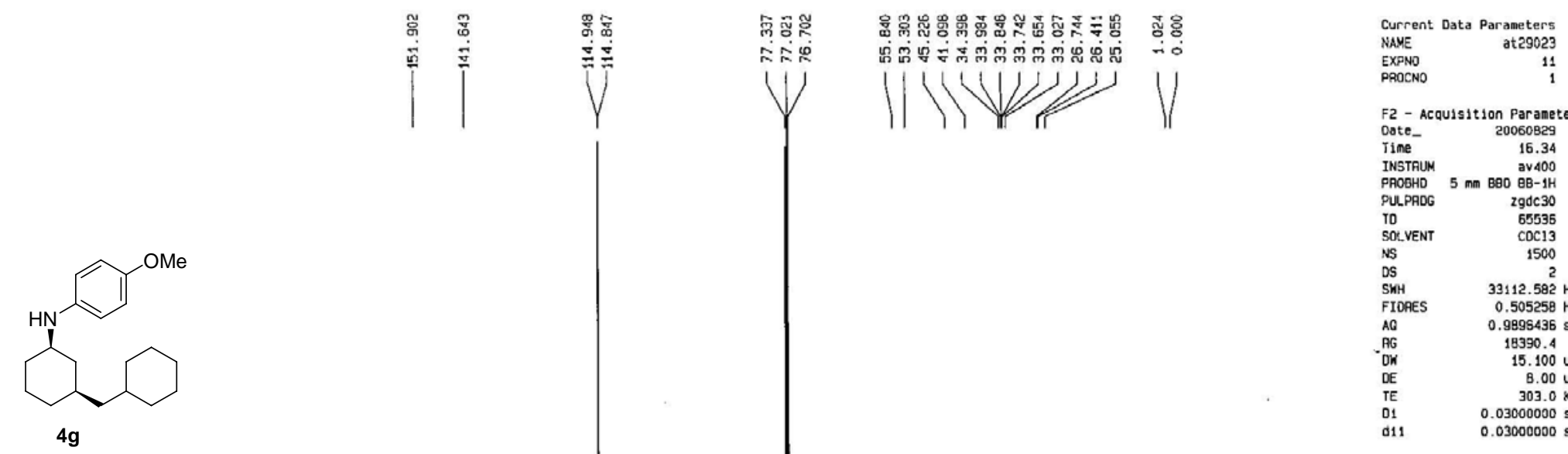

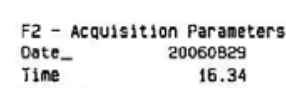

$$
\begin{array}{lr}
\text { Time } & 16.34 \\
\text { INSTFUM } & \text { av400 } \\
\text { PROBHD } & 5 \mathrm{~mm} \mathrm{BOO} 8 \mathrm{~B}-1 \mathrm{H}
\end{array}
$$

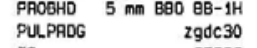

To

SOL.VENT
NS

DS $\quad \begin{aligned} & 1500 \\ & 2\end{aligned}$

$\begin{array}{lr}\text { SWH } & 33112.582 \mathrm{~Hz} \\ \text { FIORES } & 0.505258 \mathrm{~Hz}\end{array}$

$\begin{array}{ll}\text { AG } & 0.9895436 \mathrm{sec} \\ \text { AG } & 18390.4\end{array}$

$\begin{array}{lr}15.100 \mathrm{usec} \\ \mathrm{OW} & B .00 \mathrm{usec} \\ \mathrm{DE} & 0.0000 \mathrm{sec}\end{array}$

$\begin{array}{lr}\text { TE } & 303.0 \mathrm{~K} \\ 01 & 0.0300000 \mathrm{sec} \\ 01 & 0.0300000 \mathrm{sec}\end{array}$

NUC1 CHaNeL 41 1......-

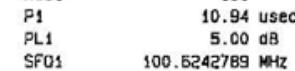

CPOPAG

$\begin{array}{lc}\text { CPOPAG2 } & \text { Naltz16 } \\ \text { NUC2 } & 1 \mathrm{H} \\ \text { PCPO2 } & 90.00 \text { usec } \\ \text { PL2 } & 0.00 \mathrm{~dB}\end{array}$

$\begin{array}{lr}\text { PL2 } & 0.00 \mathrm{~dB} \\ \text { PL12 } & 19.75 \mathrm{~dB} \\ \text { SF02 } & 400.1324710 \mathrm{MHz}\end{array}$

F2 - Processing parameters

$\begin{array}{ll}\mathrm{SI} & 32768 \\ \mathrm{SF} & 100.6127673 \mathrm{MHz}\end{array}$

$\begin{array}{lc}\text { SHF } & 100.6127673 \mathrm{MHz} \\ \text { HOH } & \mathrm{EH} \\ \text { SSB } & 0 \\ \text { LE } & 1.00 \mathrm{~Hz} \\ \text { GQ } & 0 \\ \text { PC } & 2.00\end{array}$

10 NWA plot parameters
CX $22.00 \mathrm{~cm}$

$\begin{array}{lr}C X & 22.00 \mathrm{~cm} \\ C Y & 15.00 \mathrm{~cm} \\ C Y & 250.000 \mathrm{pm}\end{array}$

$\begin{array}{lr}F_{F 1 P} & 15.00 \mathrm{~cm} \\ F 1 & 250.000 \mathrm{ppm} \\ F_{12} & 25153.19 \mathrm{~Hz}\end{array}$

$\begin{array}{ll}\text { F2P } & -30.000 \mathrm{ppm} \\ \text { F2 } & -3018.38 \mathrm{~Hz} \\ \text { PPMCM } & 12.72727 \mathrm{ppm}\end{array}$

$12.72727 \mathrm{ppm} / \mathrm{cm}$
$1280.52612 \mathrm{~Hz} / \mathrm{cm}$

zho-za-436b-cis 

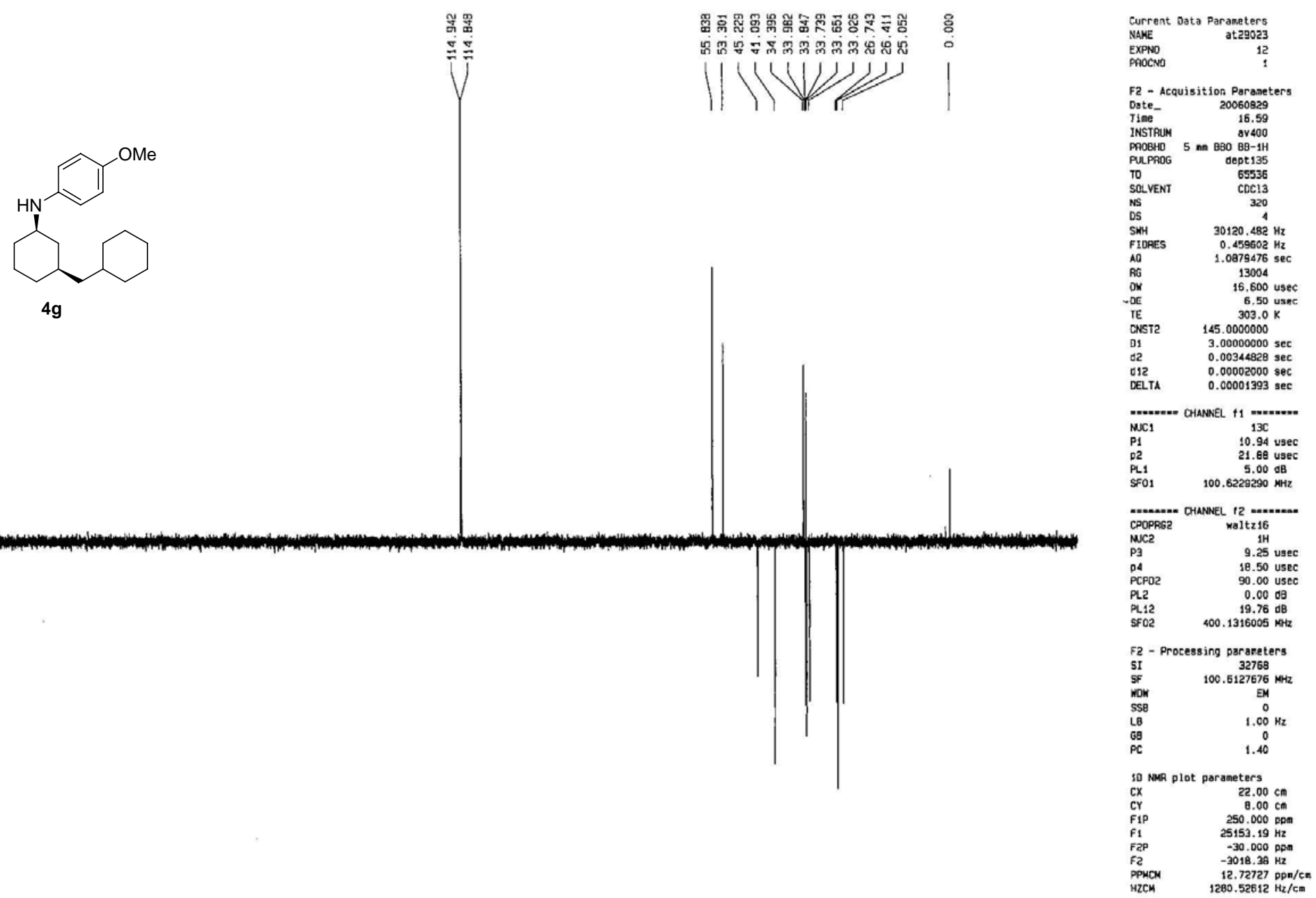

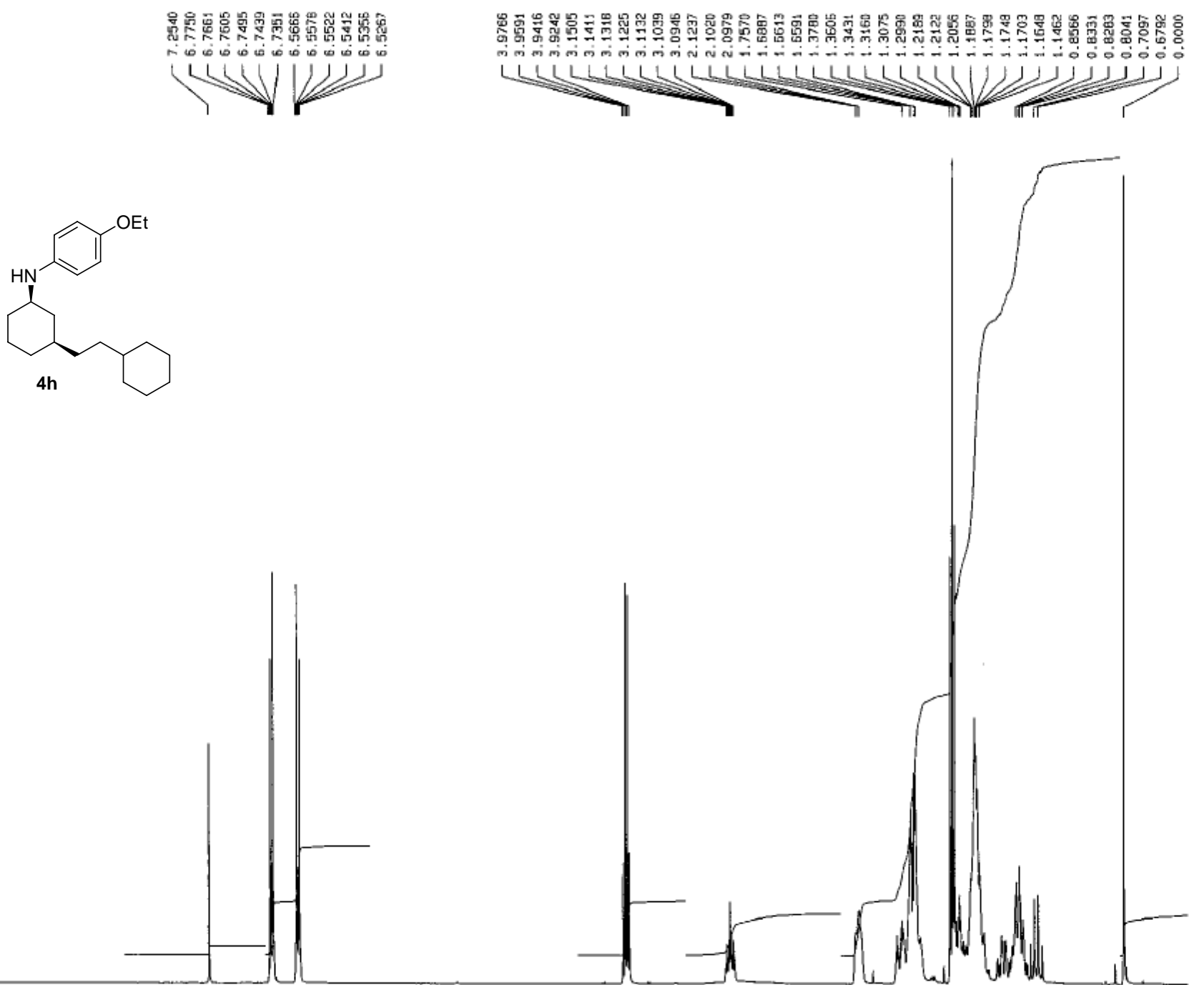

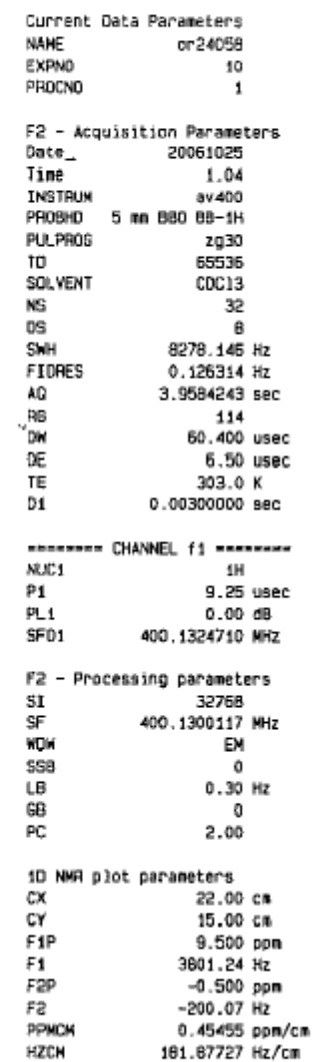

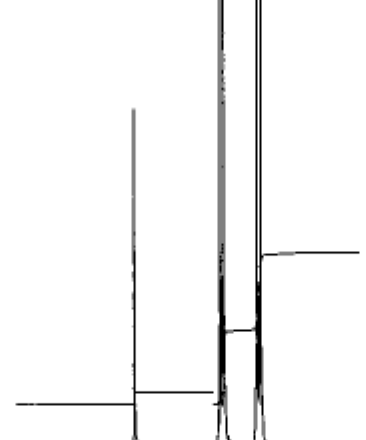



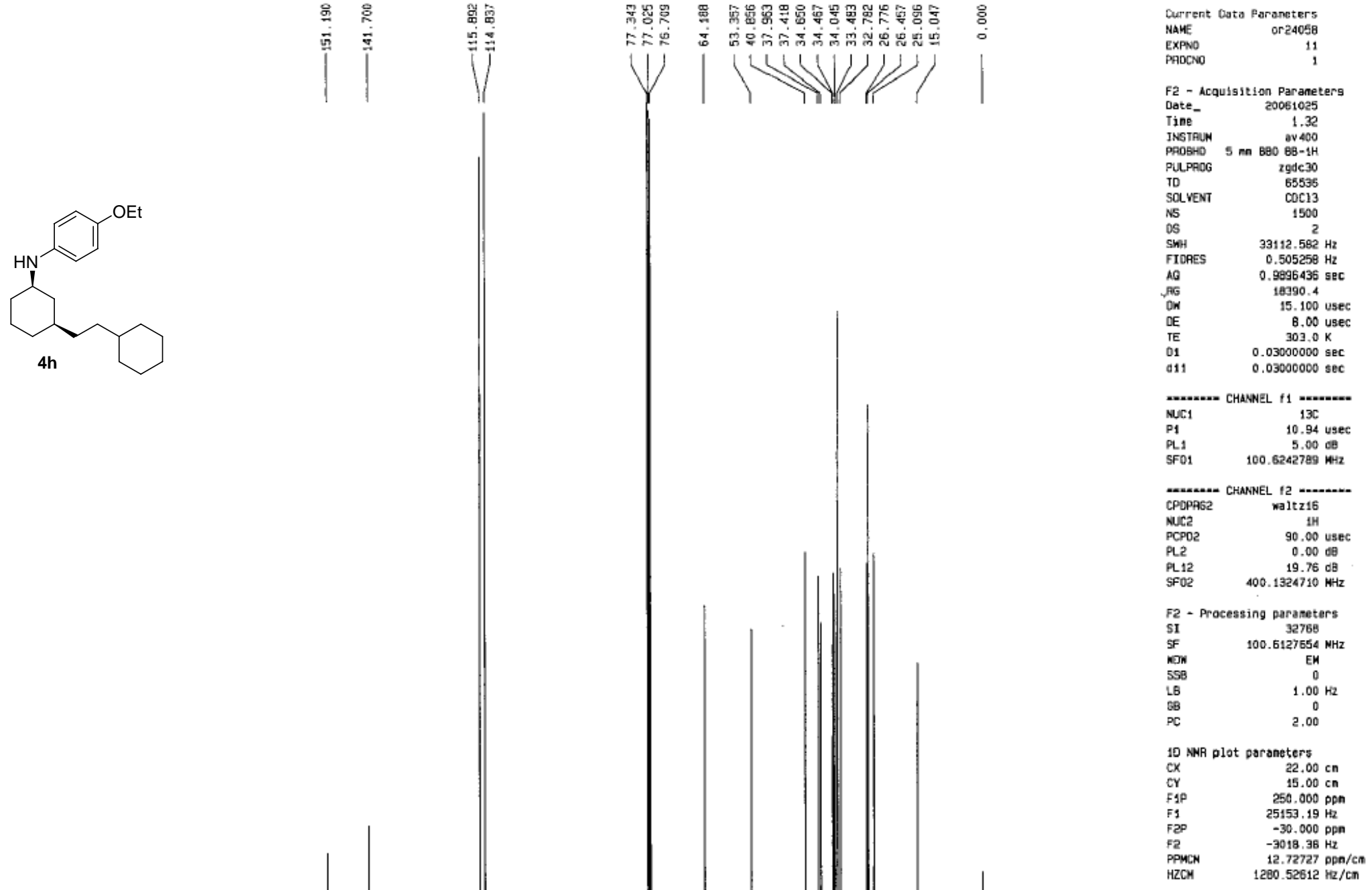

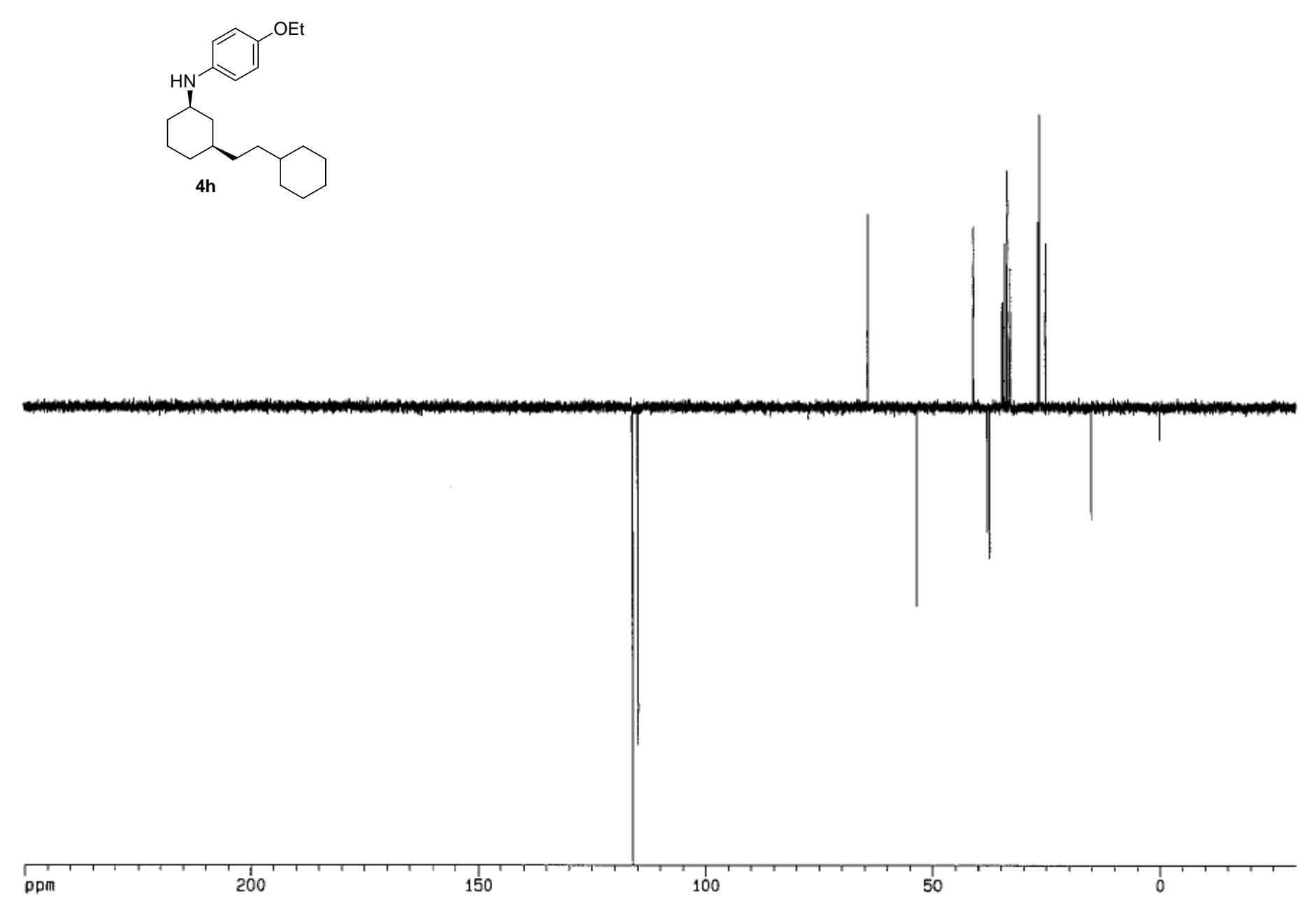

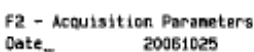

Oate-

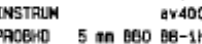

Pup proce 5 opetti35

10
SOLVENT
ML

Sol 1.20 .482 Hz

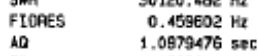

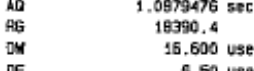

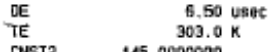

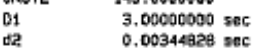

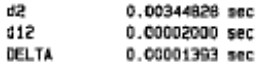

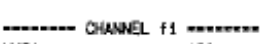

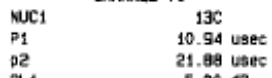

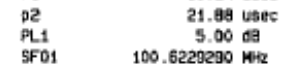

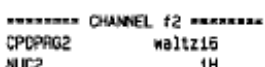

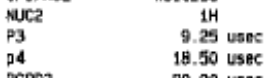

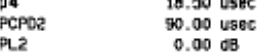

P P 1212

F2 - Processing aar aneters

st $\quad 100.61272755)$

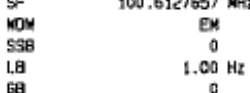

${ }_{\mathrm{PC}} \quad 1.40$

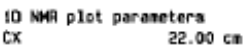

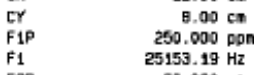

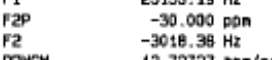

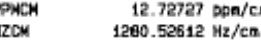



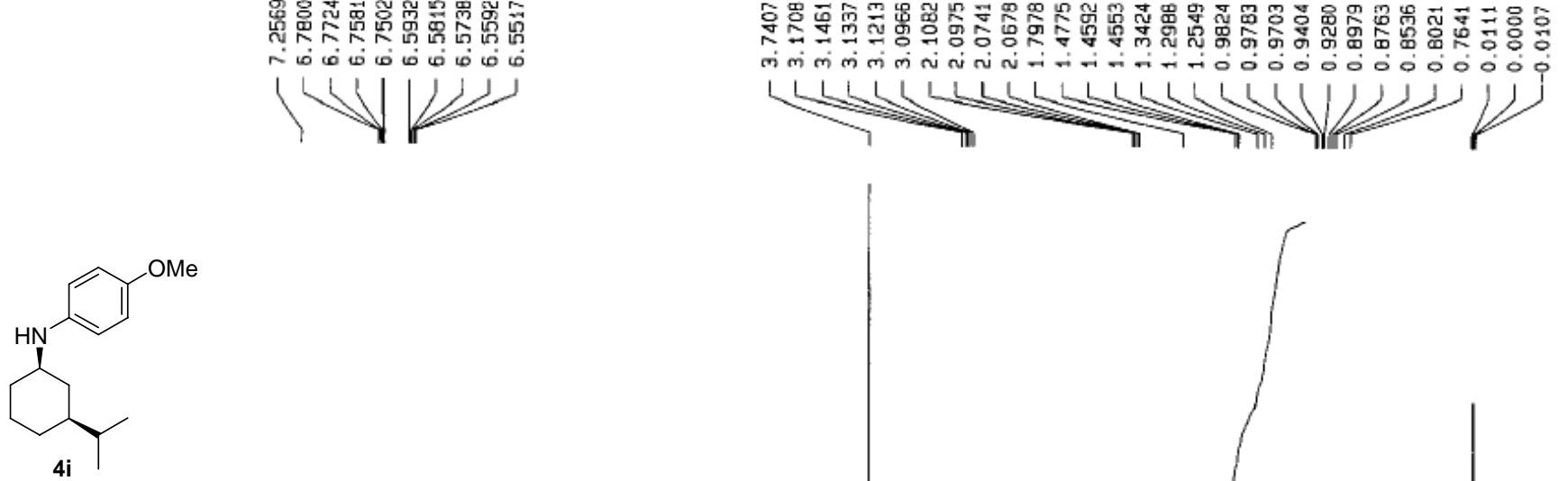

Uurrent Data Paraneters NANE PAOCNO au29058
10
1

F2 - Acquisition Paraneters Date_ 20050930 INSTRUM dox $\quad 450$ PROBHD 5 ma ONP $1 \mathrm{HH}$ PUPPAOG $\quad 2930$ $\begin{array}{ll}\text { TO } & 32766 \\ \text { SOLVENT } & \text { CDC13 }\end{array}$

SWH

$\begin{array}{ll}\text { SWH } & 6172.839 \mathrm{~Hz} \\ \text { FIDRES } & 0.188380 \mathrm{~Hz}\end{array}$

AQ $2.6542580 \mathrm{sec}$

$\begin{array}{ll}R G & 362 \\ \text { OW } & \text { B1.000 use }\end{array}$

DE 4.50 usec

$01 \quad 3.00000000 \mathrm{~K}$

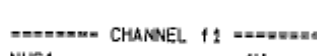

NuC1 $\quad 1 \mathrm{H}$

$P_{1} \quad 7.56$ usec

$\begin{array}{lr}\text { PL1 } & -6.00 \mathrm{~dB} \\ \text { SF01 } & 300.1318534 \mathrm{NFHz}\end{array}$

F2 - Processing oeraneters

SI

$\begin{array}{ll}\text { SI } & 16384 \\ \text { SF } & 300.1300073\end{array}$

$\begin{array}{ll}\text { HOW } & \text { EM } \\ \text { SSB } & 0\end{array}$

$\begin{array}{lc}\mathrm{LB} & 0.30 \mathrm{~Hz} \\ \mathrm{~GB} & 0\end{array}$

10 NMR plot parameters

$\begin{array}{ll}\mathrm{CX} & 22.00 \mathrm{~cm} \\ \mathrm{cY} & 15.00 \mathrm{~cm}\end{array}$

CY $\quad 15.00 \mathrm{~cm}$

$\begin{array}{lr}F 1 P & 9.500 \mathrm{ppm} \\ F 1 & 2851.24 \mathrm{~Hz}\end{array}$

$\begin{array}{ll}F_{2 P} & -0.500 \mathrm{ppm} \\ \mathrm{F2} & -150.07 \mathrm{~Hz}\end{array}$

$\begin{array}{lr}{ }_{22} & -150.07 \mathrm{~Hz} \\ \mathrm{PPMCM} & 0.45455 \mathrm{ppm} / \mathrm{cm} \\ \mathrm{HZCM} & 136.42273 \mathrm{~Hz} / \mathrm{Cm}\end{array}$

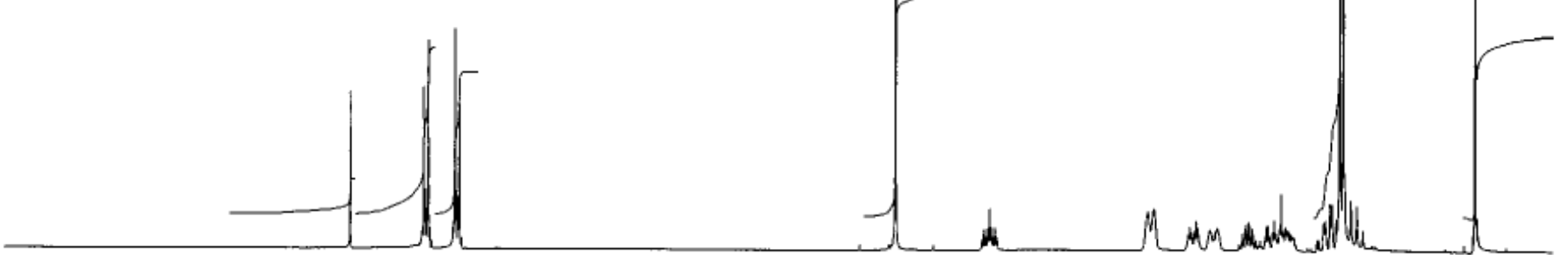

$136,42273 \mathrm{~Hz} / \mathrm{cm}$ 

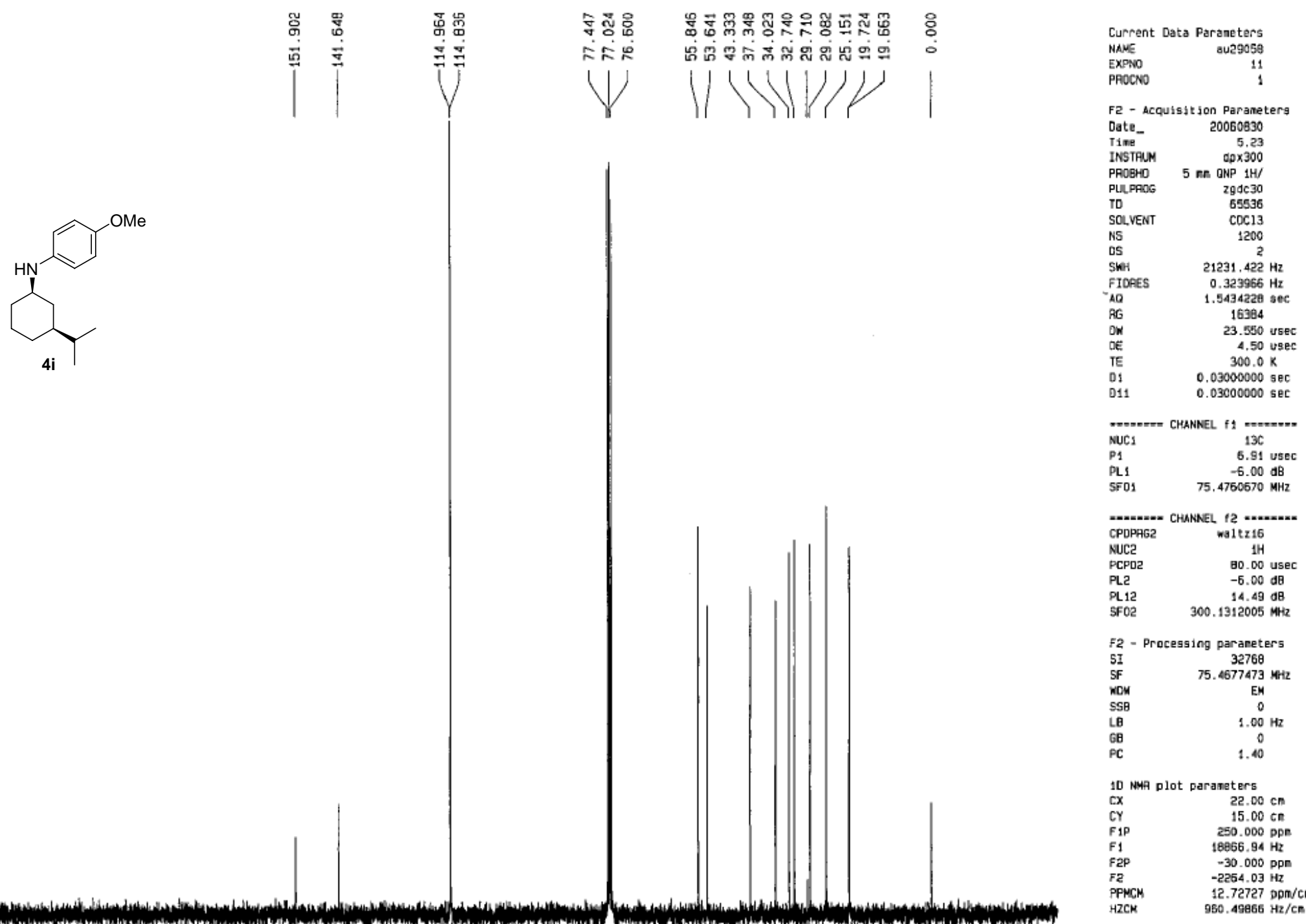

10 NuA plot paraneters

$\begin{array}{lr}\mathrm{CX} & 22.00 \mathrm{~cm} \\ \mathrm{CY} & 15.00 \mathrm{cR} \\ \mathrm{Fip} & 250.000 \mathrm{ppR}\end{array}$

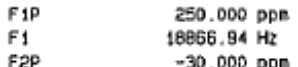

$\begin{array}{ll}F 2 P & -30.000 \mathrm{ppn} \\ F 2 & -2254.03 \mathrm{~Hz}\end{array}$

$\begin{array}{ll}\text { PPNCM } & 12.72727 \mathrm{ppm} / \mathrm{Cn} \\ \mathrm{HZCM} & 950.49866 \mathrm{~Hz} / \mathrm{Cm}\end{array}$

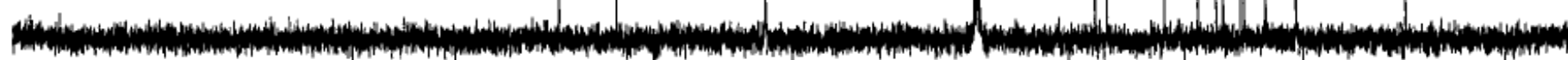




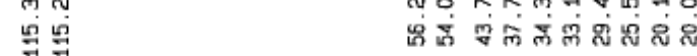

(1)

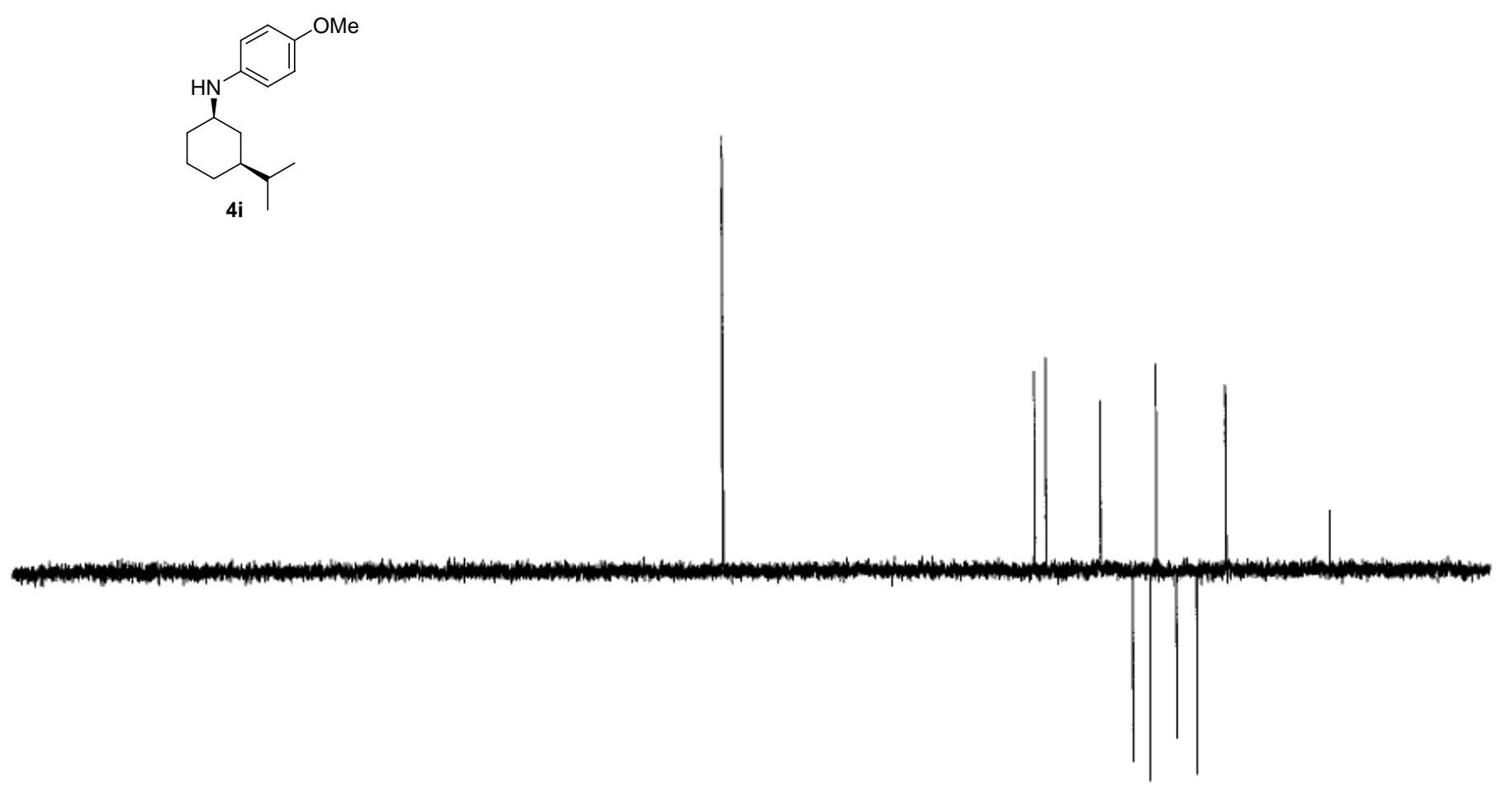

Current Dato Paraneters

EXPNO

F2 - Acquisition Parareters
Dete 20060830

Tinse-

$\begin{array}{lr}\text { Tíne } & 5.39 \\ \text { INSTRUM } & 0 \times 300\end{array}$

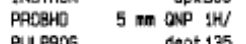

\begin{tabular}{lr} 
PULPA06 & dept 135 \\
TO & 65536 \\
\hline
\end{tabular}

SOLVEN

D5 150

$\begin{array}{ll}\text { SWH } & 21231.422 \mathrm{~Hz} \\ \text { FIOFES } & 0.323965 \mathrm{~Hz}\end{array}$

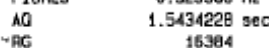

$\begin{array}{ll} & \\ & 23.550 \\ D E & 4.50\end{array}$

TE $300.0 \mathrm{~K}$

$\begin{array}{ll}2.00000000 \mathrm{sec} \\ 02 & 0.00357143 \mathrm{sec} \\ 02 & 0.00002000 \mathrm{sec}\end{array}$

DELTA $\quad 0.0000008 B 0 \mathrm{sec}$

NUC:

$\begin{array}{lr}\mathrm{P}_{1} & 13 \mathrm{C} \\ \mathrm{P}_{2} & 6.91 \text { usec } \\ \mathrm{P} & 13.82 \text { usec }\end{array}$

$\begin{array}{lr}\mathrm{P} 2 & 13.82 \text { usec } \\ \mathrm{PL1} & -5.00 \text { ob } \\ \text { SF01 } & 75.4750670 \mathrm{NHz}\end{array}$

CPPPAG2 OHANvEL 12 ....nn......

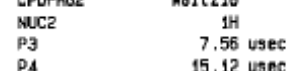

$\begin{array}{ll}p_{4} & 15.12 \text { usec } \\ \text { perpor } & 80.00 \text { usec }\end{array}$

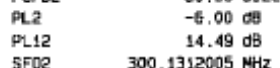

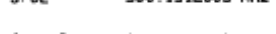

F2 - Processing paraneters
S1
32768

$\begin{array}{ll}51 & 32768 \\ 5 F & 75.4677190 \\ \mathrm{FHH}\end{array}$

$\begin{array}{lc}\text { NOW } & \text { EK } \\ \text { SSB } & 0 \\ \text { LB } & 1.00 \mathrm{HZ} \\ \text { GB } & 0.00\end{array}$

10 NiHa plot paraseters
CX $22.00 \mathrm{cn}$

$\begin{array}{lc}\mathrm{CX} & 22.00 \mathrm{~cm} \\ \mathrm{CY} & 6.50 \mathrm{~cm} \\ \mathrm{~F} 1 \mathrm{P} & 250.000 \mathrm{pmm} \\ \mathrm{F} 1 & 18666.93 \mathrm{~Hz} \\ \mathrm{~F} 2 \mathrm{P} & -30.000 \mathrm{pom} \\ \mathrm{F2} & -22564.03 \mathrm{~Hz} \\ \mathrm{PPHCH} & 12.72727 \mathrm{pom} / \mathrm{cm} \\ \mathrm{HZCH} & 960.49923 \mathrm{~Hz} / \mathrm{cm}\end{array}$

zho-za-440b-cis 


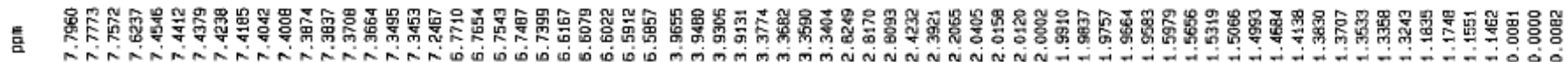

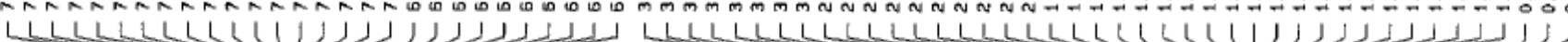
L จา
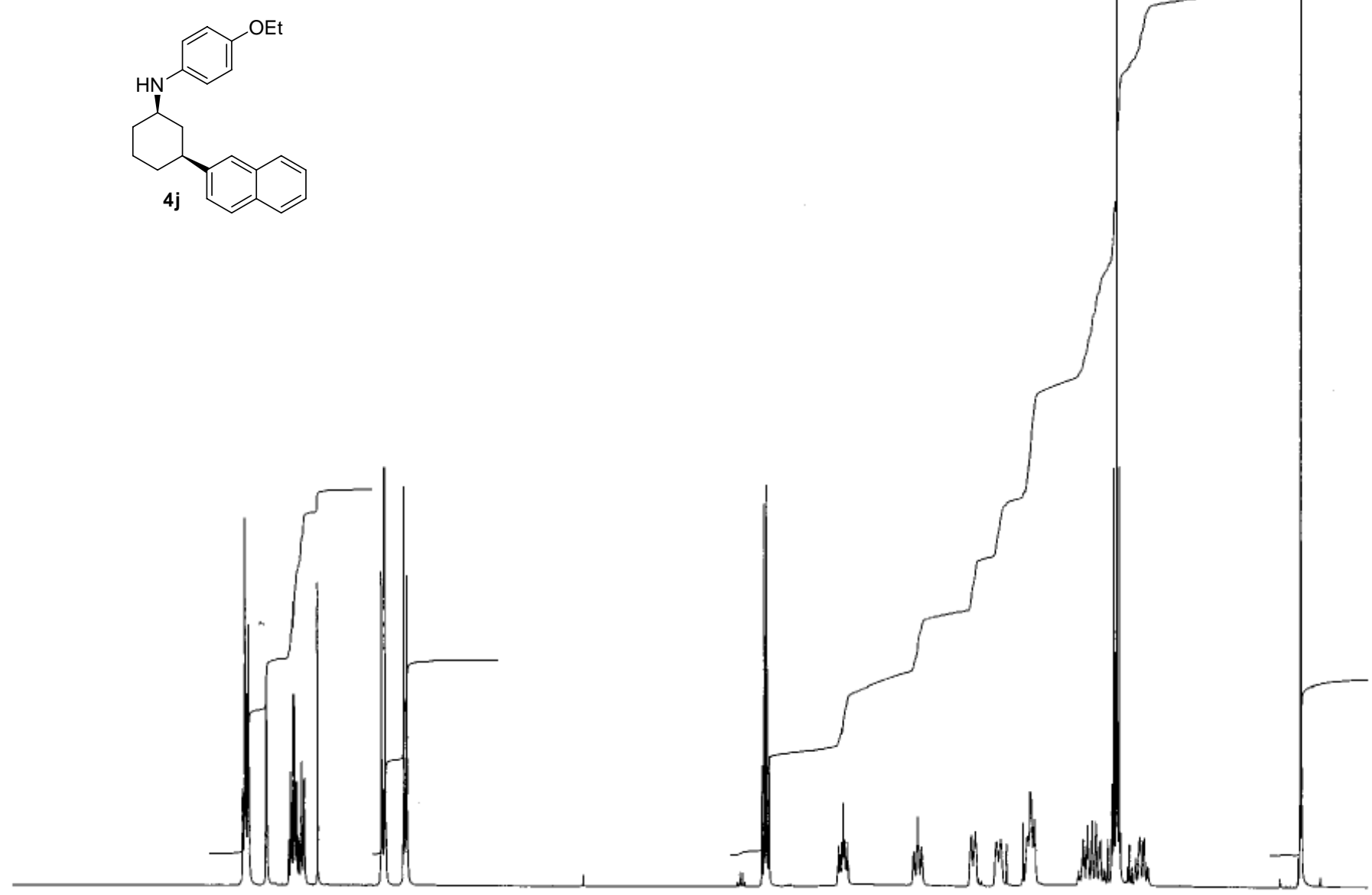

Current Data Paraneters
Nawe

Exeno

F2 - Acquisition Paraneters

Tate-

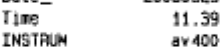

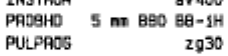

2930
PUPAPOS
TO
SOYVEYT

紧

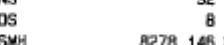

$\begin{array}{ll}\text { FIOFES } & 0.126314 \mathrm{~Hz} \\ A B & 3.9584243 \mathrm{sec}\end{array}$

RS
DW
DE

$\begin{array}{ll} & 6.50 \text { use } \\ \text { TE } & 303.0 \mathrm{~K} \\ \text { TE } & 0.0030000\end{array}$

NUC1

$\begin{array}{lr}\text { P1 } & 9.25 \text { usec } \\ \text { PL1 } & 0.00080 \\ \text { SF01 } & 400.1324710 \text { Netz }\end{array}$

$\begin{array}{lc}\text { F2 - Processing paraneters } \\ \text { SI } & 32768 \\ \text { SF } & 400.1300150\end{array}$

$\begin{array}{lc}\text { NOW } & \text { EM } \\ \text { SSB } & 0 \\ \text { LB } & 0.30 \mathrm{~Hz}\end{array}$

$G 8$

10 Nur plot paraneters

$\begin{array}{ll}\mathrm{CX} & 22.00 \mathrm{~cm} \\ \mathrm{CY} & 15.00 \mathrm{~cm} \\ \mathrm{Fy} & 5.500 \mathrm{pm}\end{array}$

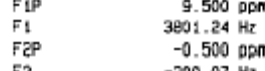

PPMCH $\quad-200.07 \mathrm{~Hz}$

PFMCH
$\mathrm{HZCH}$
$181.87727 \mathrm{~Hz} / \mathrm{Cr}$

6

5

4

3

2

1

' 1

zho-za-430b-cis 

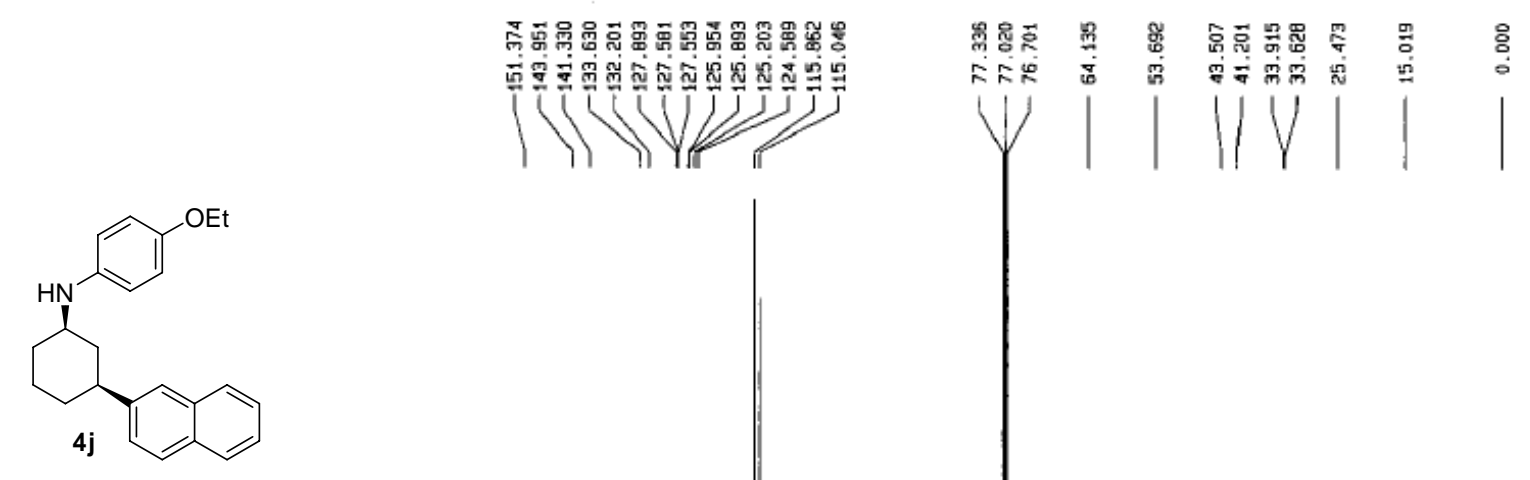

Current Data Paraneters
NaHE
EXPNO at29005

PFOCNO

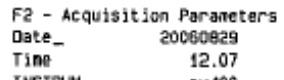

INSTTAM

PULPAOG

$\begin{array}{ll}\text { TO } & 65536 \\ \text { SOLVENT } & \text { CDC13 } \\ \text { NS } & 1500\end{array}$

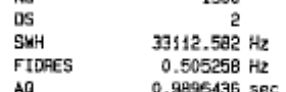

$\begin{array}{ll}A G & 16384 \\ \text { AG } & 15.100 \mathrm{usec}\end{array}$

$\begin{array}{ll}\text { DE } & 8.00 \text { usec } \\ \text { TE } & 303.0 \mathrm{~K}\end{array}$

$\begin{array}{ll}01 & 0.03000000 \mathrm{sec} \\ & \\ \mathrm{d} 11 & 0.03000000 \mathrm{sec}\end{array}$

$==3 \pi=z=$
NUC1 CHANNEL

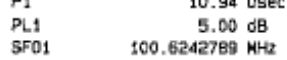

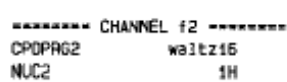

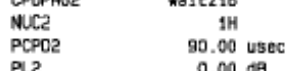

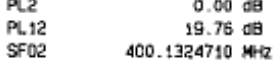

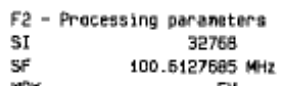

$\begin{array}{lc}\text { row } & \text { EN } \\ \text { SSB } & 0 \\ \text { LB } & 1.00 \mathrm{~Hz}\end{array}$

Ge

Nup plot paraneters

10 NuP plot parameters
CX
$22.00 \mathrm{~cm}$

$\begin{array}{lr}\text { CY } & 15.00 \mathrm{~cm} \\ \text { Fip } & 250.000 \mathrm{oph} \\ \text { F1 } & 25153.19 \mathrm{~Hz}\end{array}$

$\begin{array}{ll}\text { F2P } & -30.000 \mathrm{ppn} \\ \text { F2 } & -3015.38\end{array}$

$\begin{array}{ll}\text { F2 } & -3018.38 \mathrm{~Hz} \\ \text { PPHCM } & 12.72727 \mathrm{ppaz} / \mathrm{Cm}\end{array}$

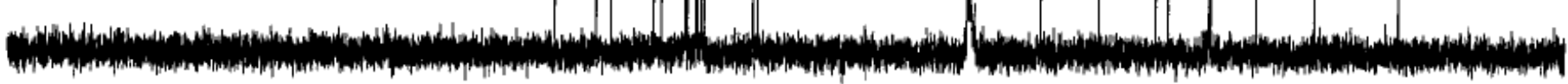


气

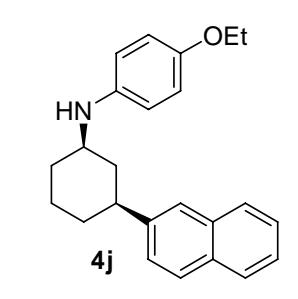

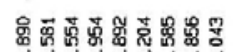

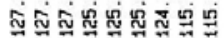

1. जि

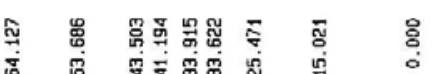

$1|1| 1$

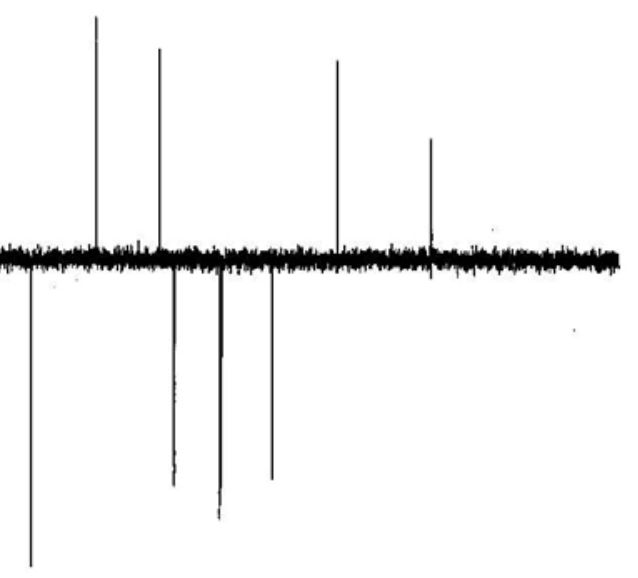

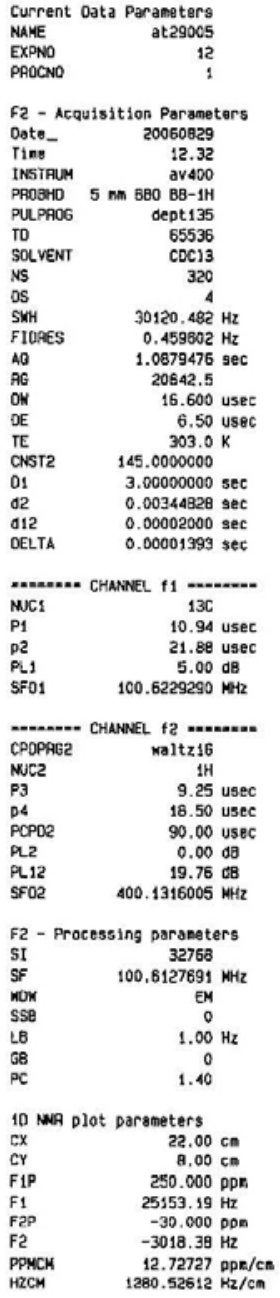

zho-za-430b-cis 


\section{๕్}
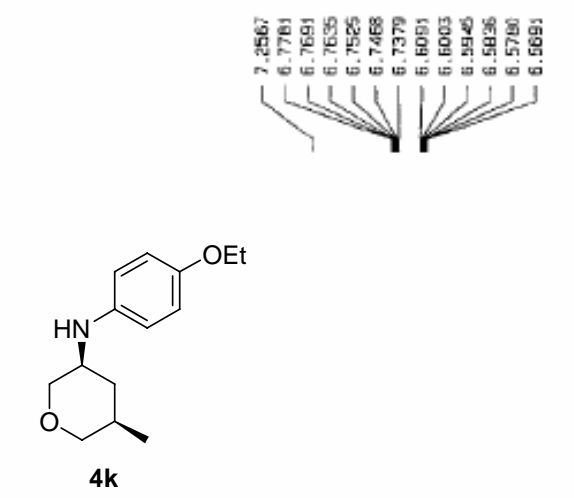

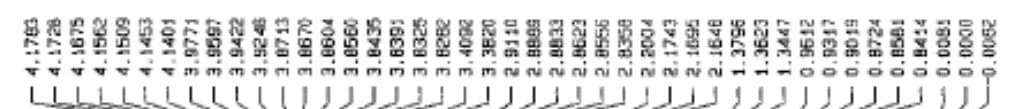

ubsuly y y y y

$4 \mathrm{k}$

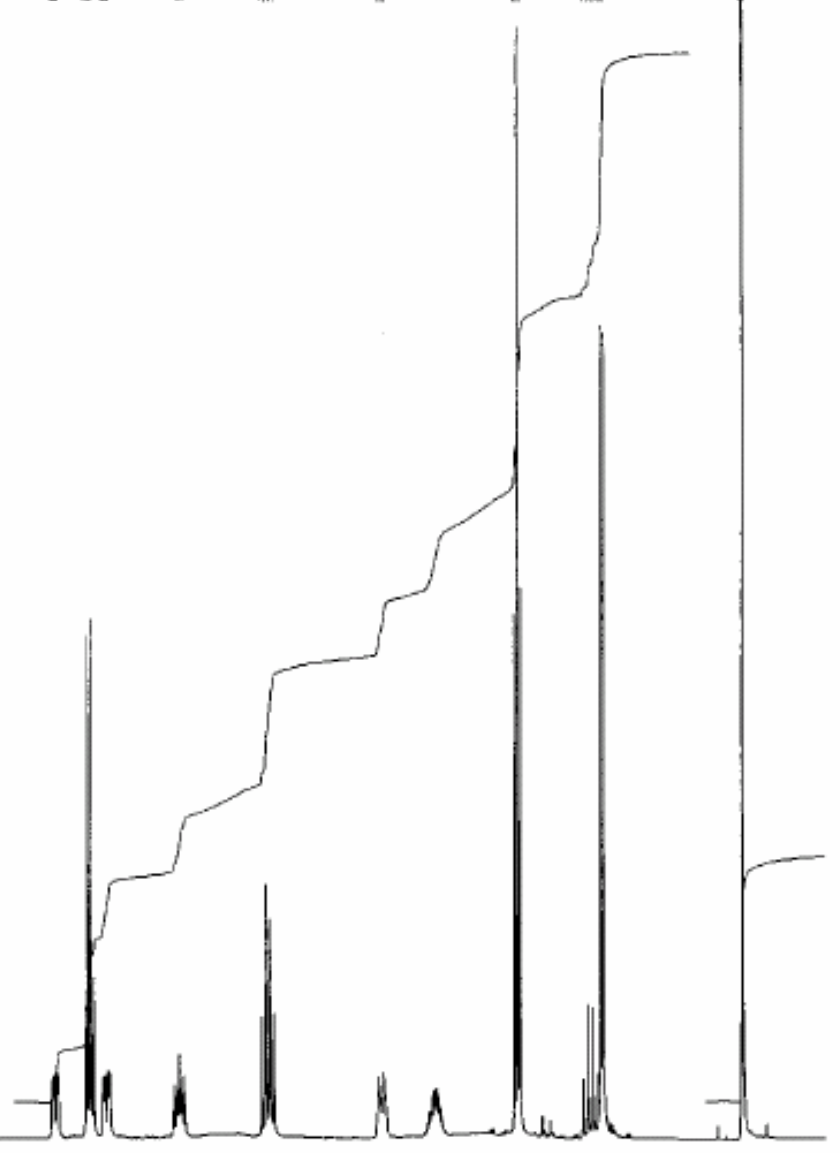

${ }_{4}^{4}$

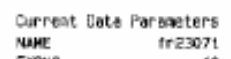

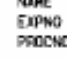

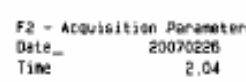

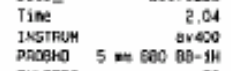

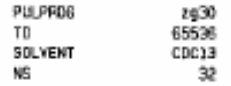

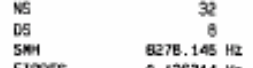

Al 0.0 .128314 Hz

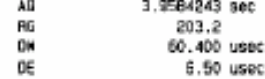

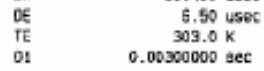

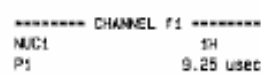

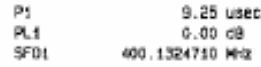

F2- Arocesessing perapeters

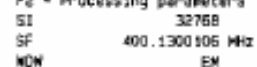

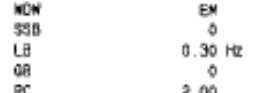

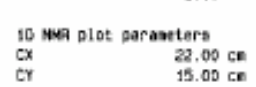

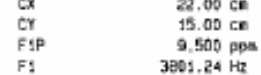

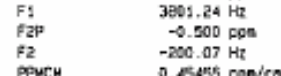

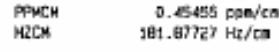

zho-zb-107-2 

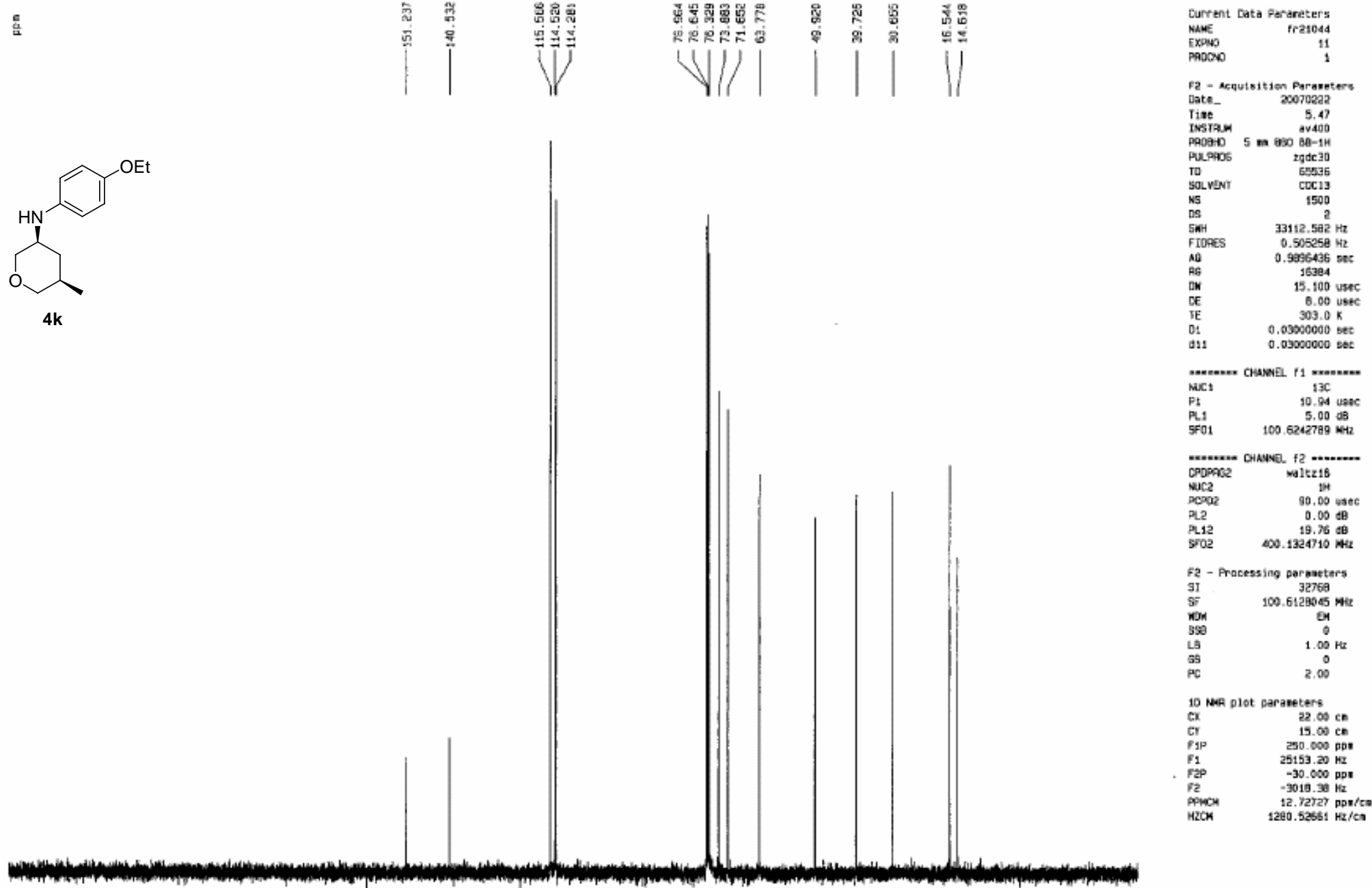


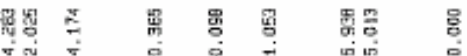

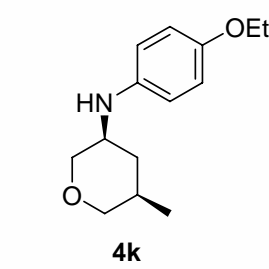

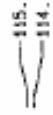

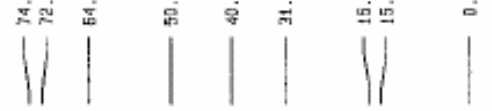

$4 \mathrm{k}$
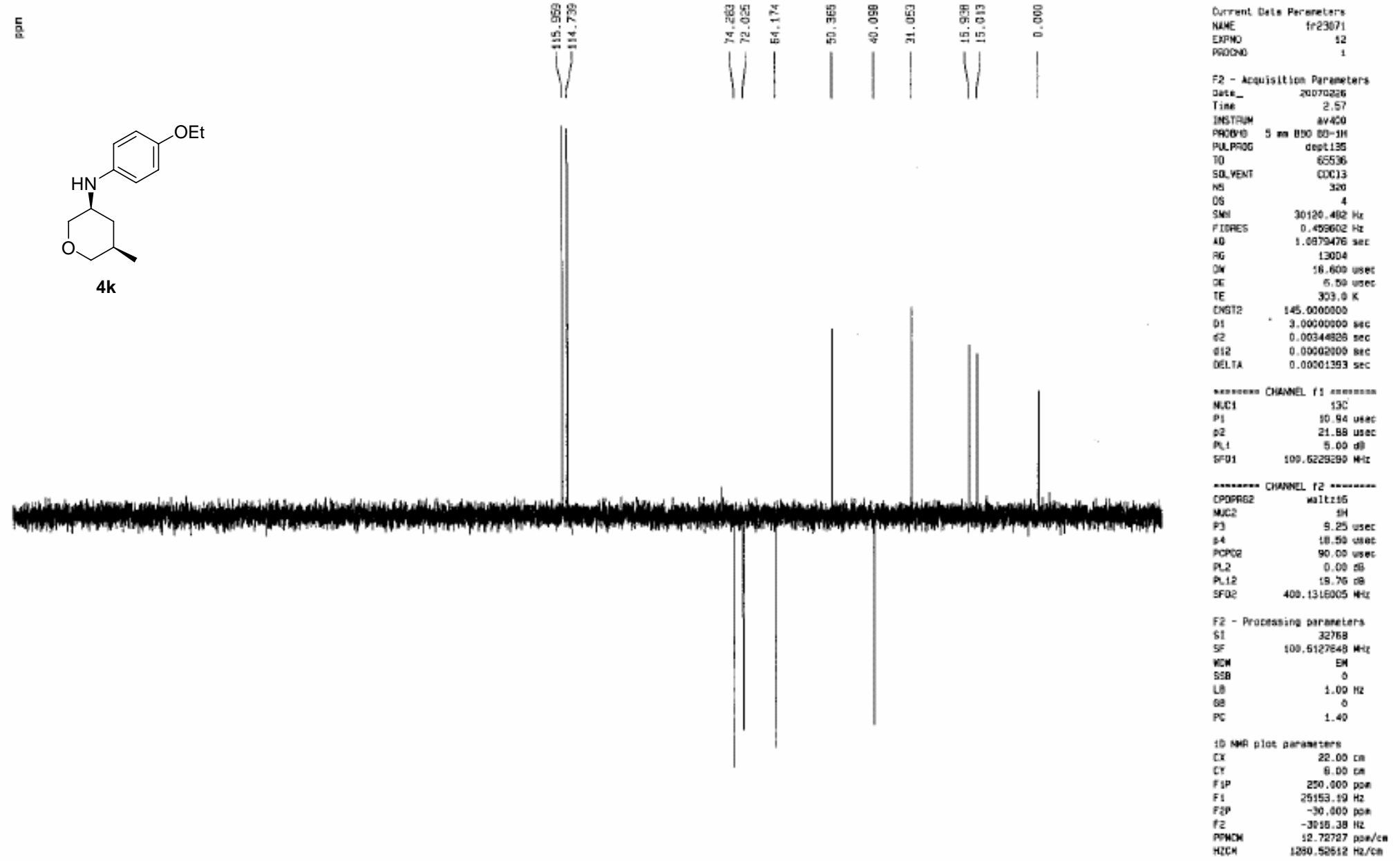

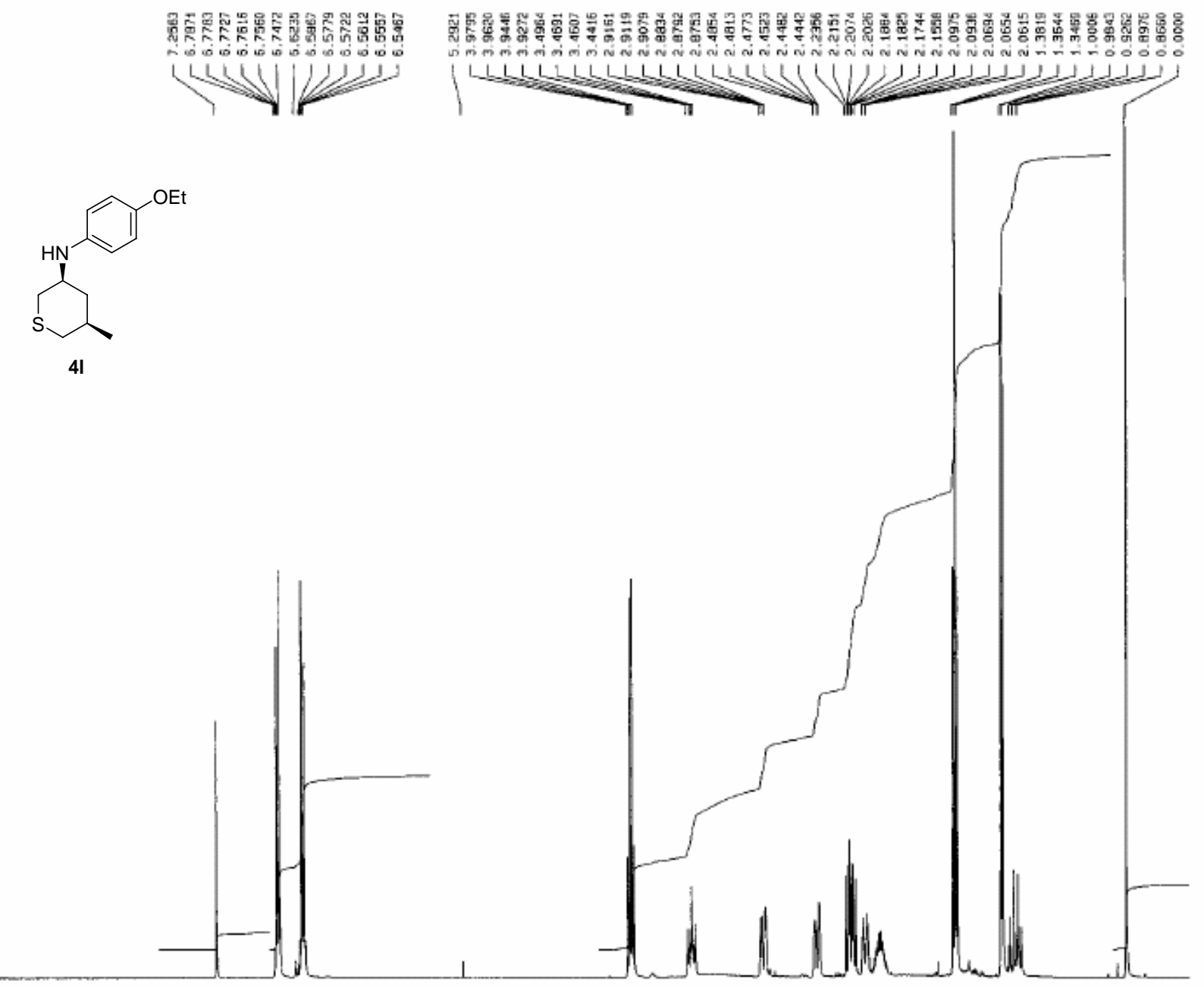

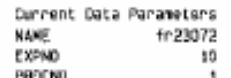

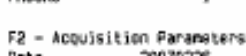

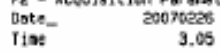

ThSTFUK

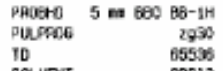

$\begin{array}{lr}\text { SOLVENT } & \text { Cac13 } \\ 156 & 32 \\ \text { OS } & 8 \\ \text { S5H } & 8279.145\end{array}$

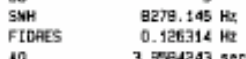

19
90
$0 \%$

TE $303.0 \mathrm{~K}$

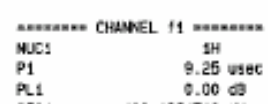

F2 - Procassing prezenters

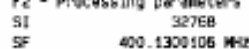

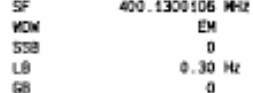

10 nea dot paravetars
$c . x$
22.00

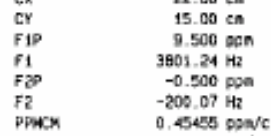

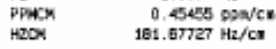

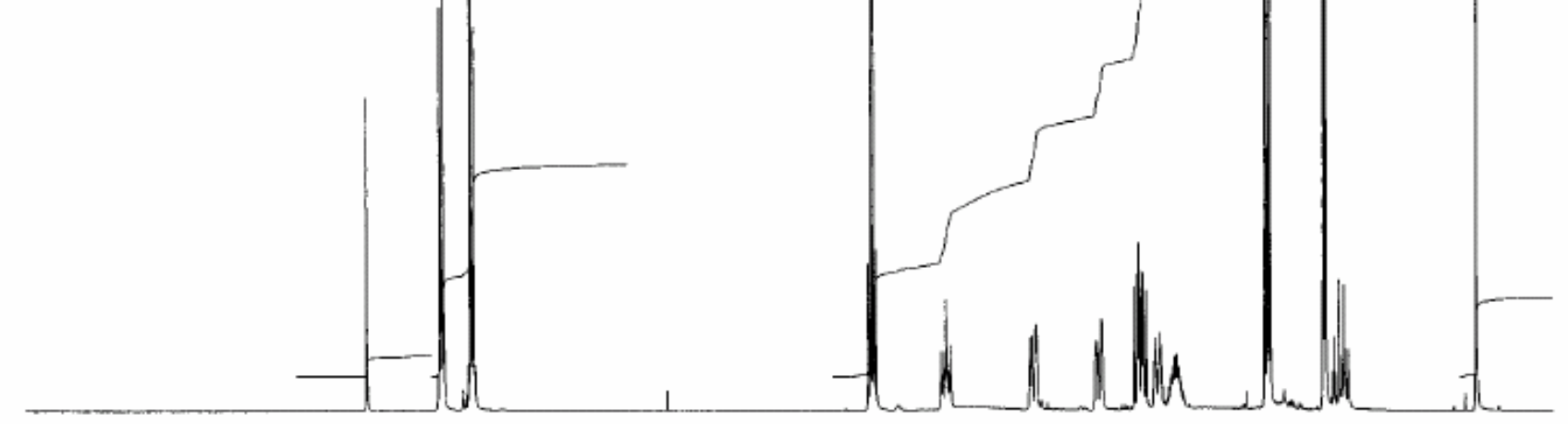

zho-zb-119-c is

8

16

5

4

2

i 

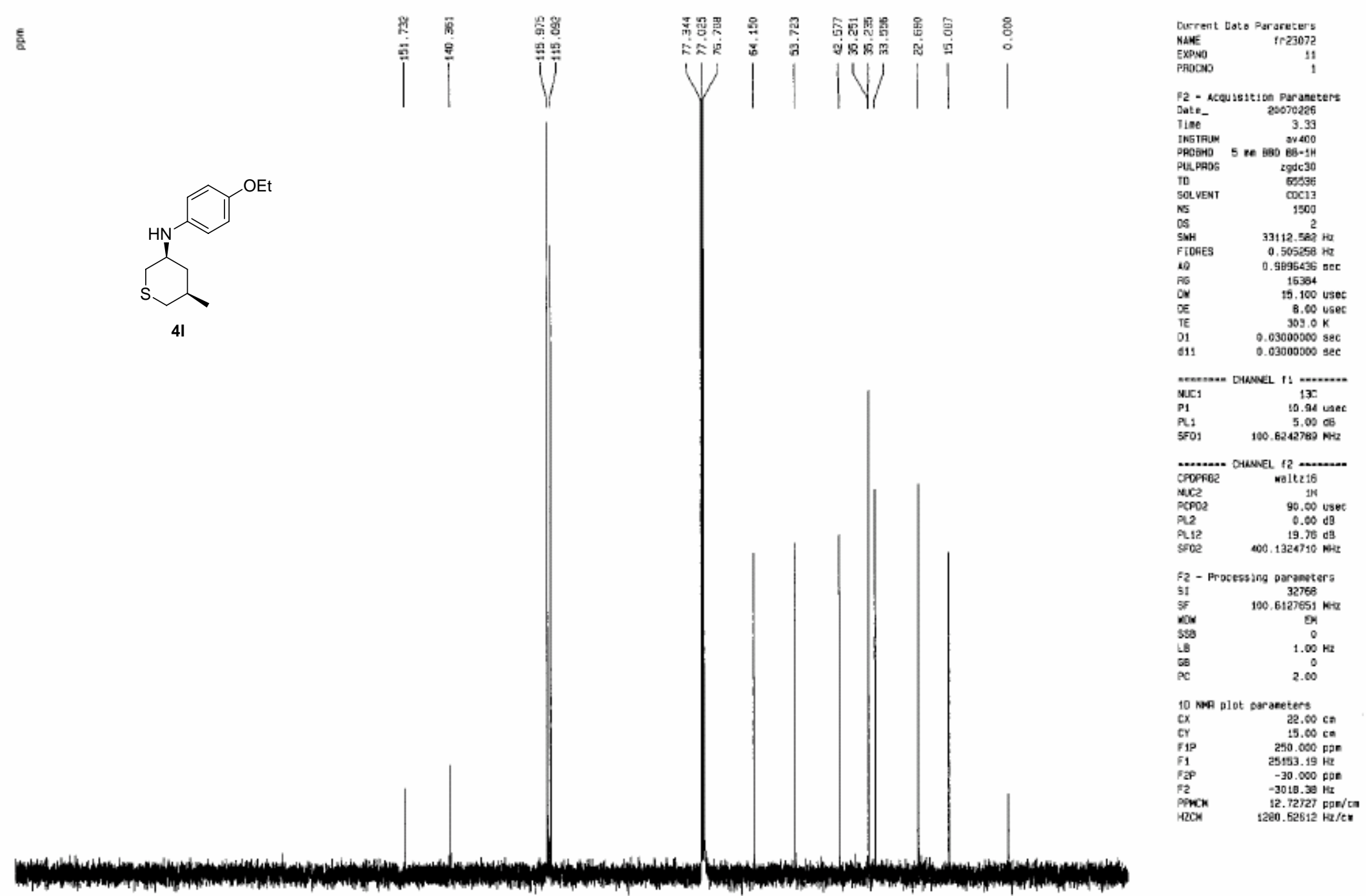

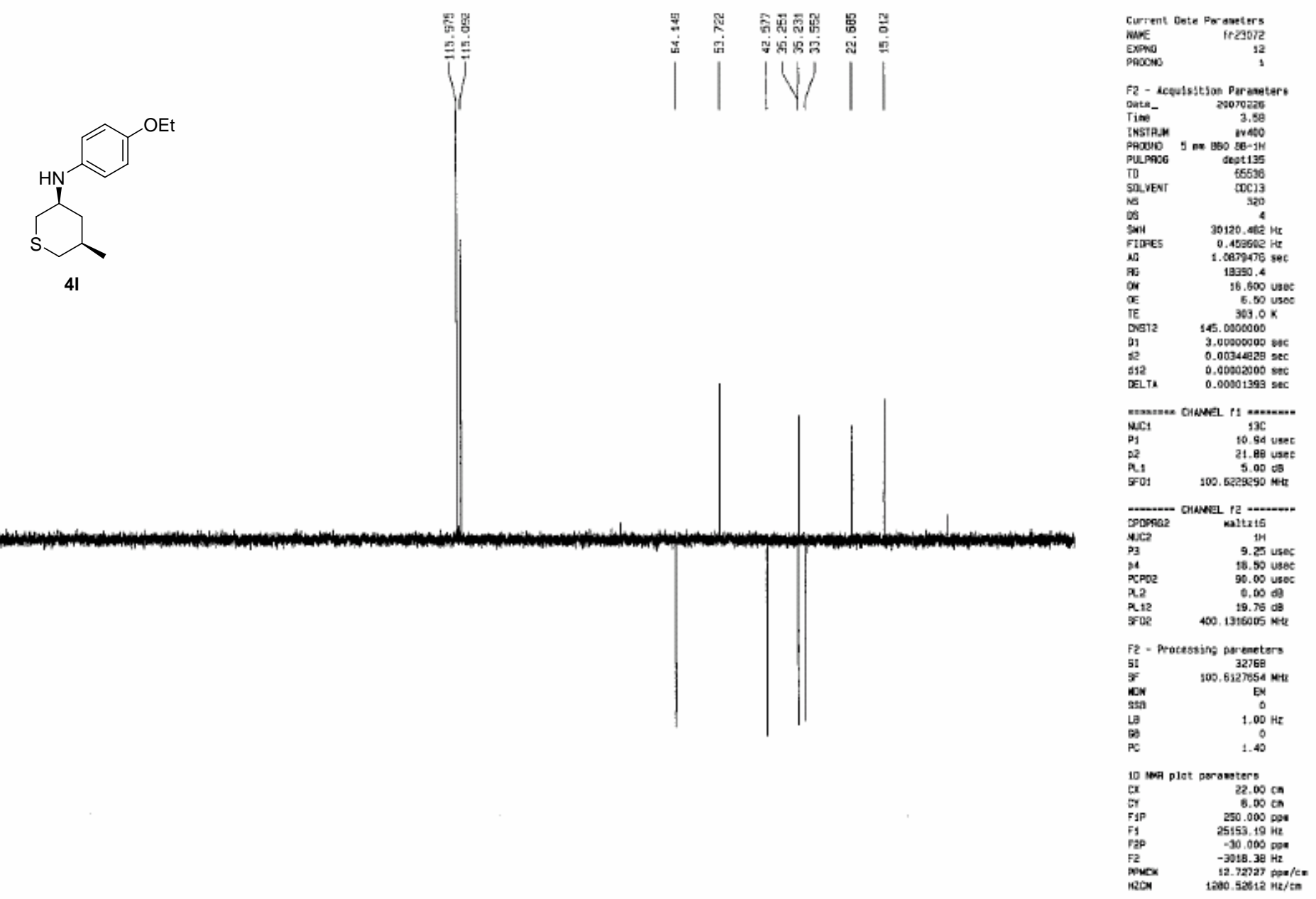


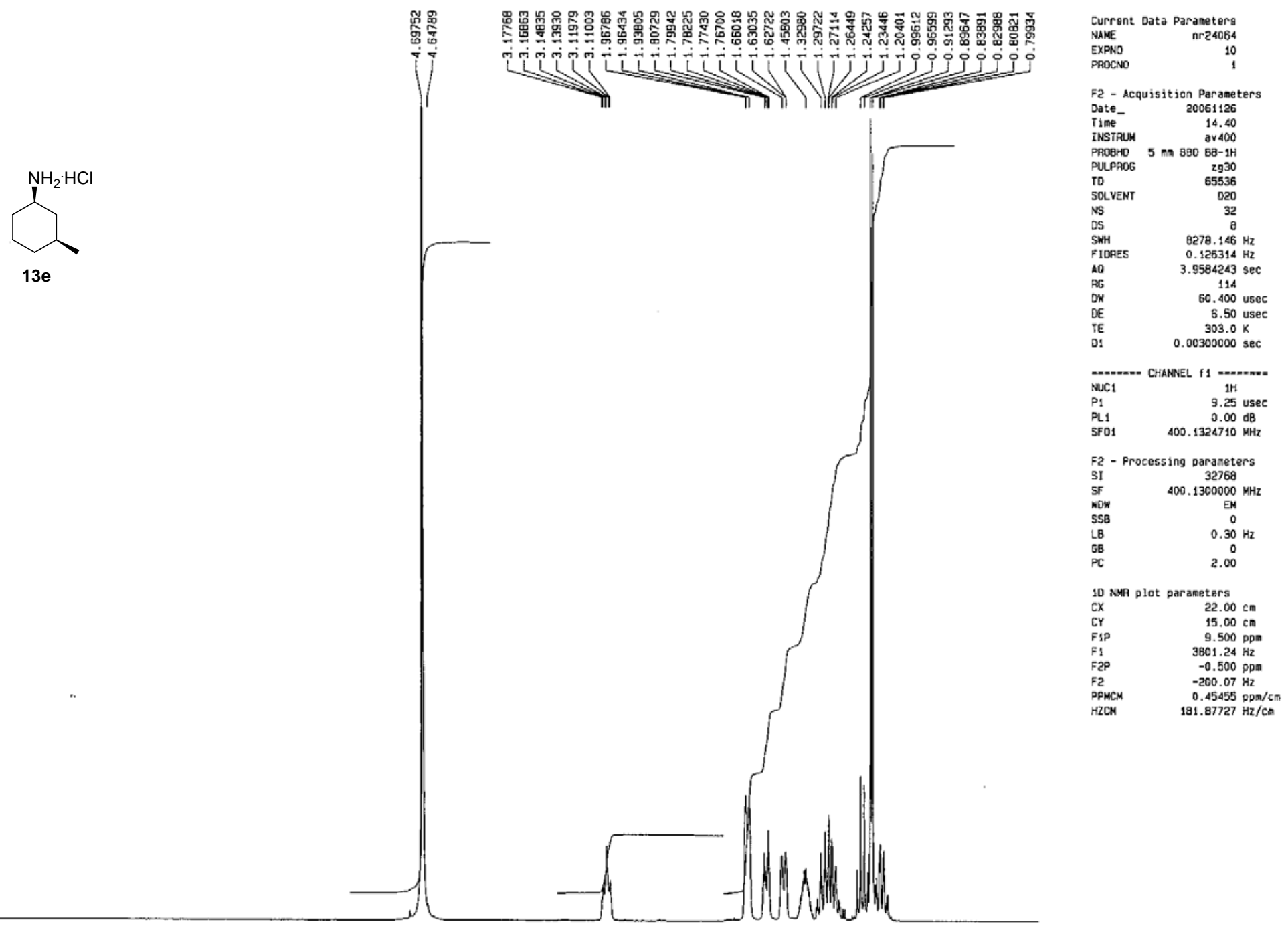

ppm

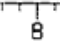

8

7

5

5

1

Tु1

zho-zb-11-h 


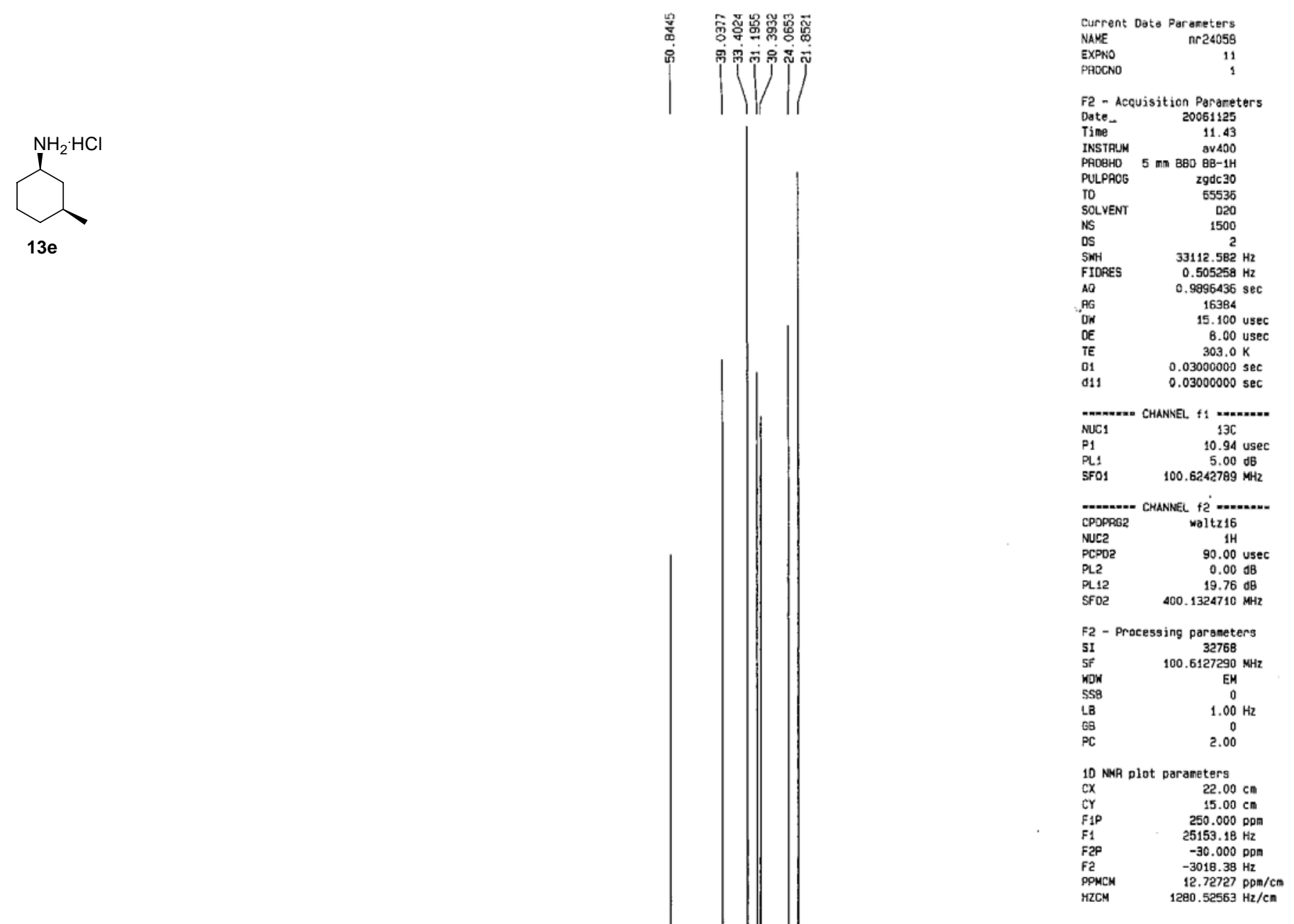

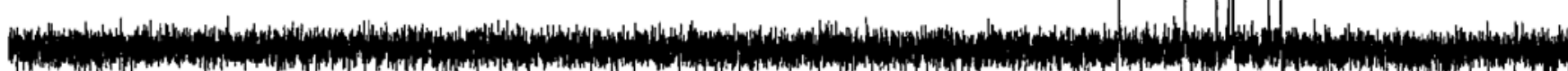
ppm 200 150 100 50 

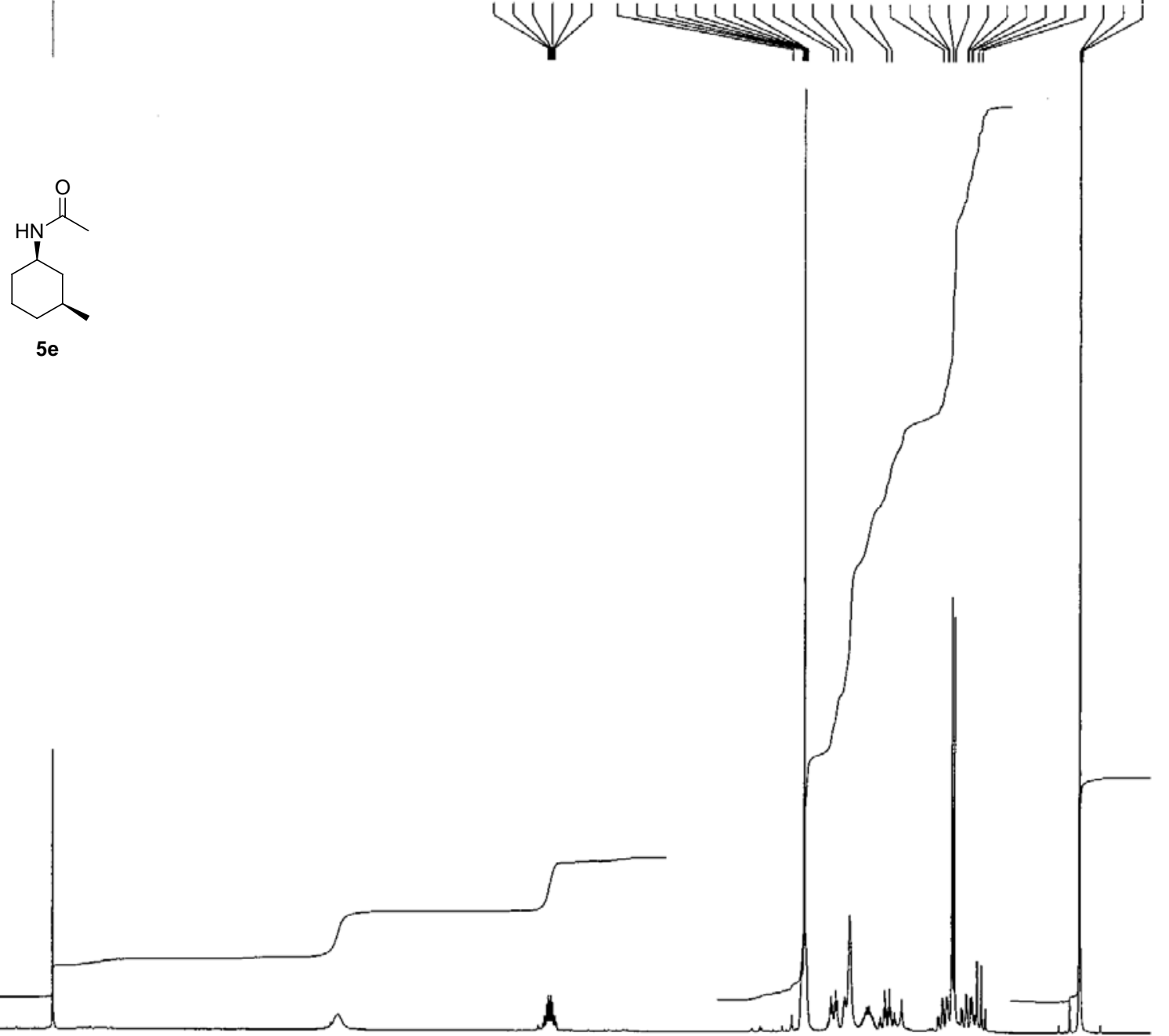

Current Data Paraneters

EXPNO

F2 - Accuisition Parometers

Date-

Tige
Twstrut
The

INSTrUM
PROOHD

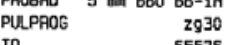

55536
SoLVENT
coc13
32

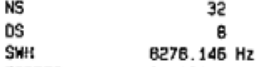

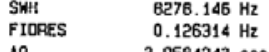

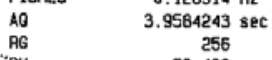

60
$D E$ TE
Tis $\quad 0.003000000 \mathrm{sec}$

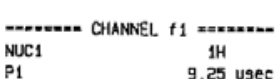

P1
PL1

F2 - Processing paraseters

SI
SF

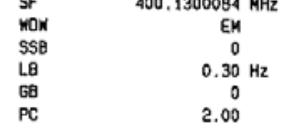

10 NHA plot parameters

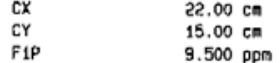

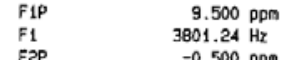

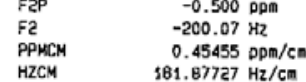

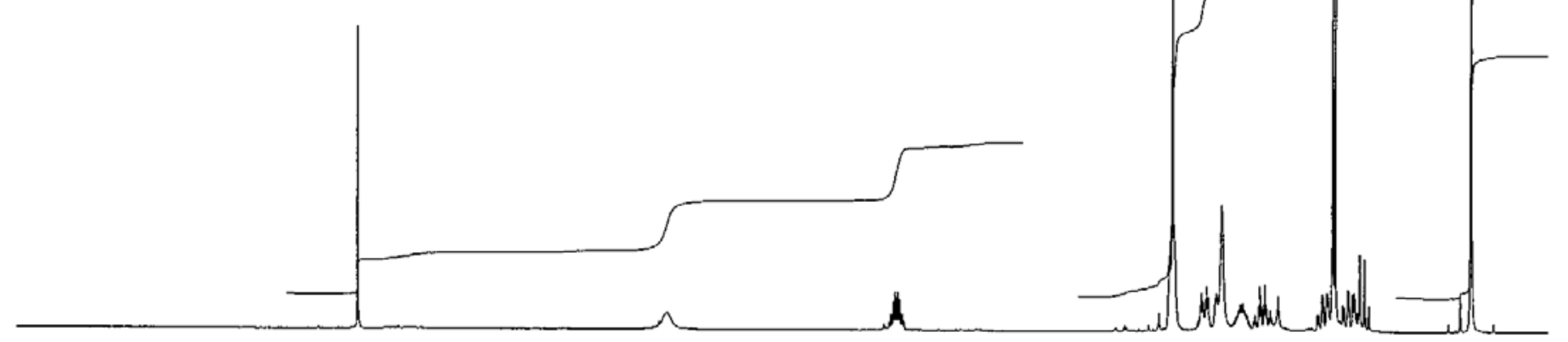




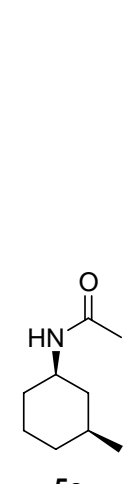

$5 e$
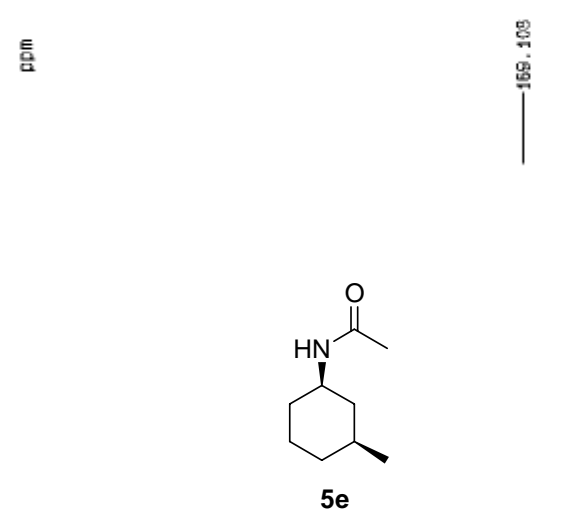

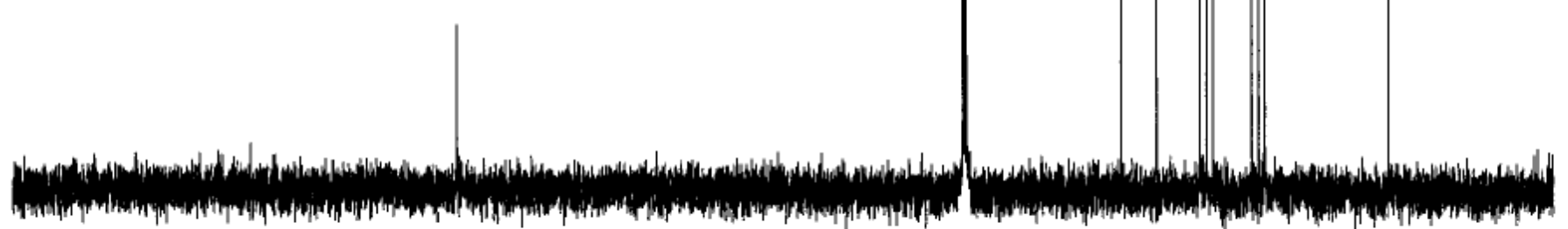

100

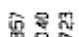

SR

W

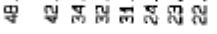

1VIV

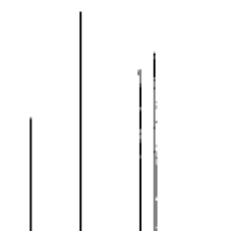

ppm
200 $150^{\circ}$

$$
\begin{aligned}
& \text { current Date Paraneters } \\
& \text { F2 - Acquistition Paraneters } \\
& \begin{array}{l}
\text { Dine } \\
\text { INSTRum }
\end{array}
\end{aligned}
$$

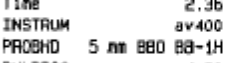

$$
\begin{aligned}
& \begin{array}{l}
\text { SOLVENT } \quad \text { CDC13 } \\
\text { NS } \\
\text { NSOO }
\end{array} \\
& \text { ShH } \quad 33112.582 \mathrm{~Hz} \\
& { }_{10} \quad 0.505258 \mathrm{~Hz} \\
& \begin{array}{l}
\text { PA } \\
\text { OW } \\
\text { DE }
\end{array} \\
& \text { IE } \quad 303.0 \mathrm{~K} \\
& \begin{array}{ll}
011 & 0.03000000 \mathrm{sec} \\
011 & 0.03000000 \mathrm{sec}
\end{array} \\
& \begin{array}{lll}
\text { NuC1 } \\
P_{1}
\end{array}
\end{aligned}
$$

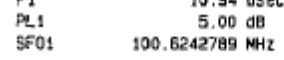

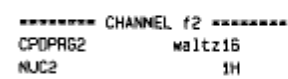

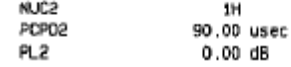

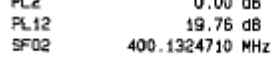

$$
\begin{aligned}
& \text { F2 - Processing paraneters } \\
& \begin{array}{ll}
\text { SI } & 32766 \\
\text { SF } & 100.6127641 \\
\mathrm{HHz} &
\end{array}
\end{aligned}
$$

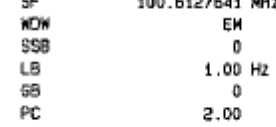

$$
\begin{aligned}
& \text { 10 NMR plot paraneters } \\
& \begin{array}{lr}
c x & 22.00 \mathrm{cn} \\
c r & 15.00 \mathrm{~cm} \\
\mathrm{~F} & 250.000 \mathrm{ppn}
\end{array}
\end{aligned}
$$

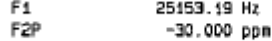

$$
\begin{aligned}
& \begin{array}{ll}
\text { F2 } & -3018.30 \mathrm{~Hz} \\
\text { PPMCM } & 12.72727 \mathrm{PDB} / \mathrm{cn} \\
\text { HZCM } & 1280.52612 \mathrm{~Hz} / \mathrm{Cn}
\end{array}
\end{aligned}
$$




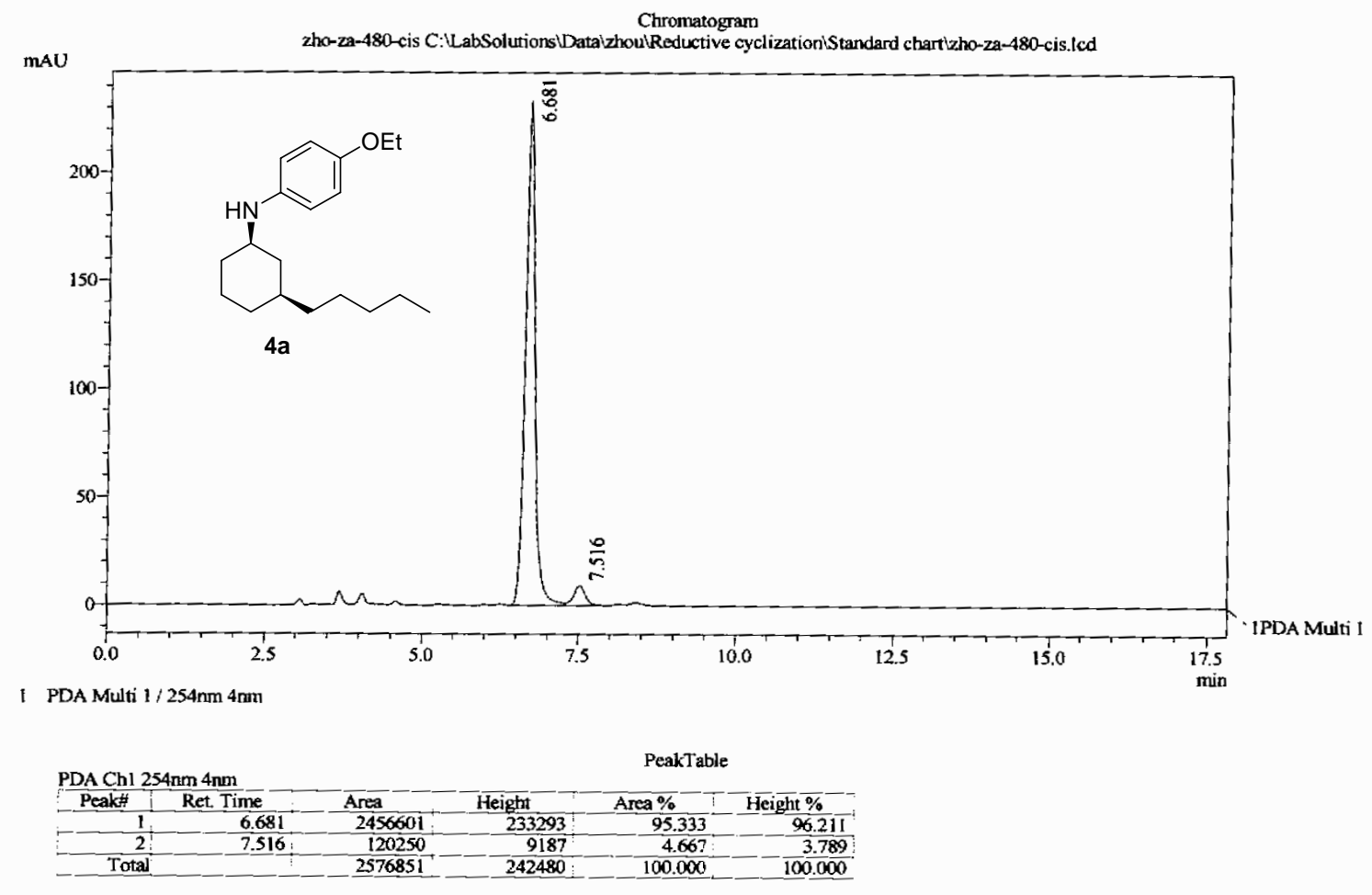

Chromatogram

zho-7a-479b-cis-rac C.LabSolutionsiDataivhou Reductive cyclizationiStankard chart'zho-za-479b-cis.lcd

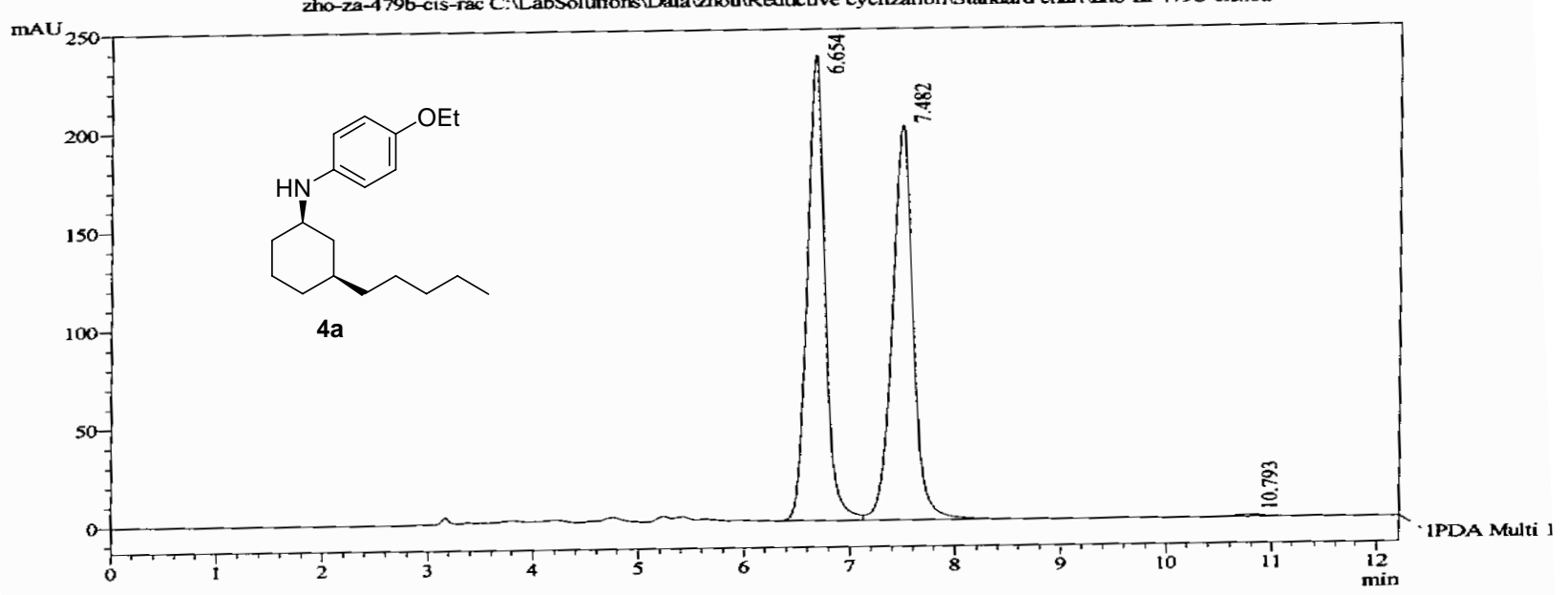

1 PDA Multi $1 / 254 \mathrm{~nm} 4 \mathrm{rum}$

PeakTable

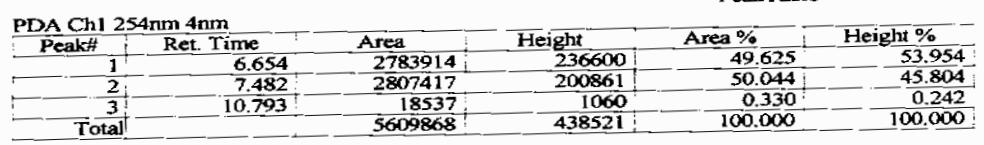


$\mathrm{mV}$

C-ILabSolutionsIDatalZhoulReductive CyclizationiStandard chartlzho-za-402b-cis.lcd

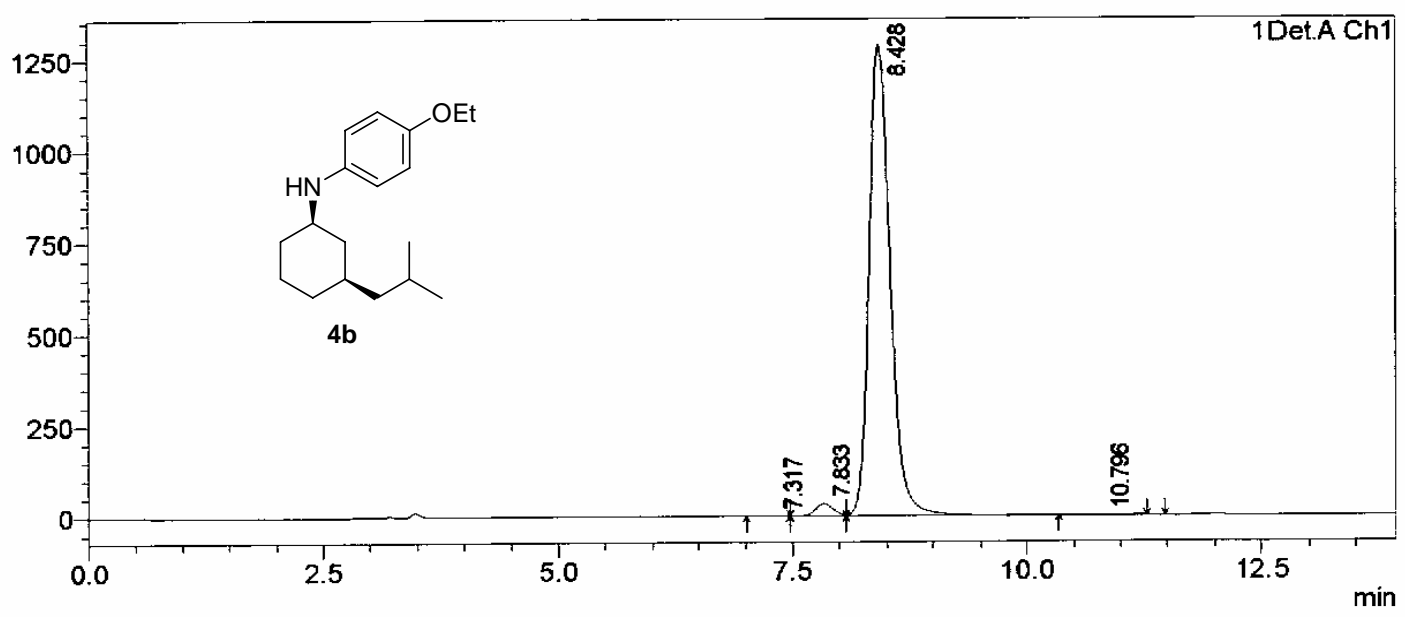

1 Det.A Ch $1 / 247 \mathrm{~nm}$

\section{<Results>}

Detector A Ch1 247nm
\begin{tabular}{|l|r|r|}
\hline Peak \# & Ret. Time & Area \% \\
\hline 1 & 7.317 & 0.016 \\
\hline 2 & 7.833 & 2.373 \\
\hline 3 & 8.428 & 97.565 \\
\hline 4 & 10.796 & 0.046 \\
\hline Total & & 100.000 \\
\hline
\end{tabular}

$\mathrm{mV}$

C:ILabSolutionsIDatalZhoulReductive CyclizationiStandard chartizho-za-402a-cis-rac.lcd

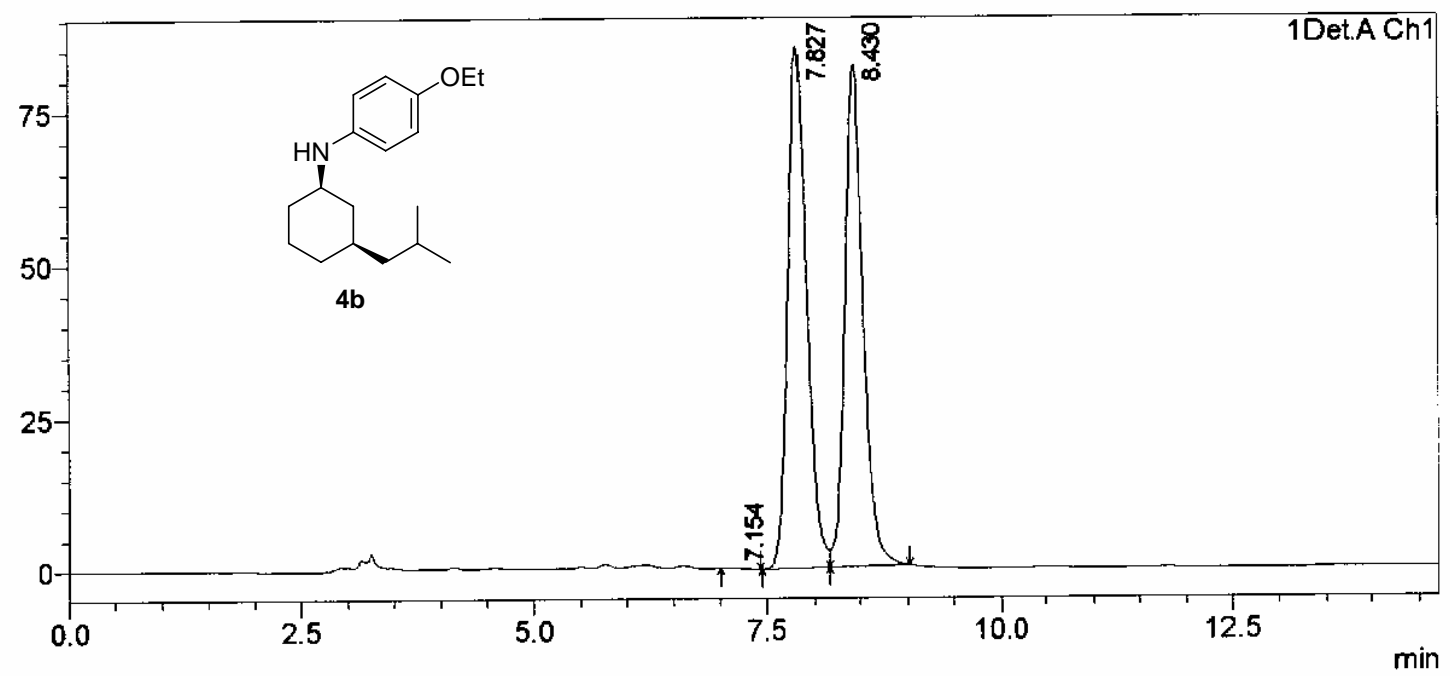

1 Det.A Ch1/247nm

\section{<Results>}

Detector A Ch1 $247 \mathrm{~nm}$
\begin{tabular}{|l|r|r|}
\hline Peak \# & Ret. Time & \multicolumn{1}{c|}{ Area \% } \\
\hline 1 & 7.154 & 0.052 \\
\hline 2 & 7.827 & 49.598 \\
\hline 3 & 8.430 & 50.350 \\
\hline Total & & 100.000 \\
\hline
\end{tabular}




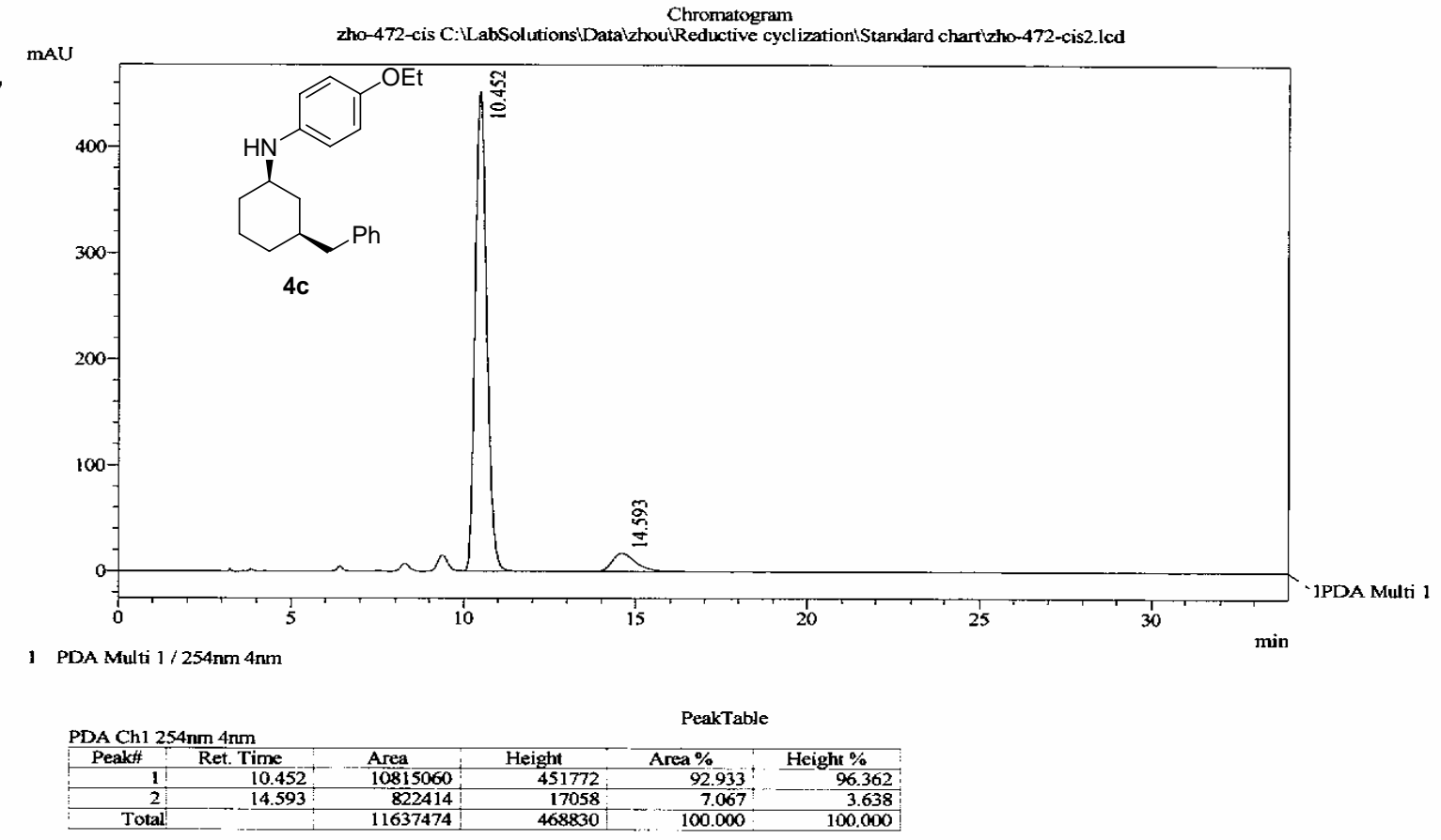

Chromatogram

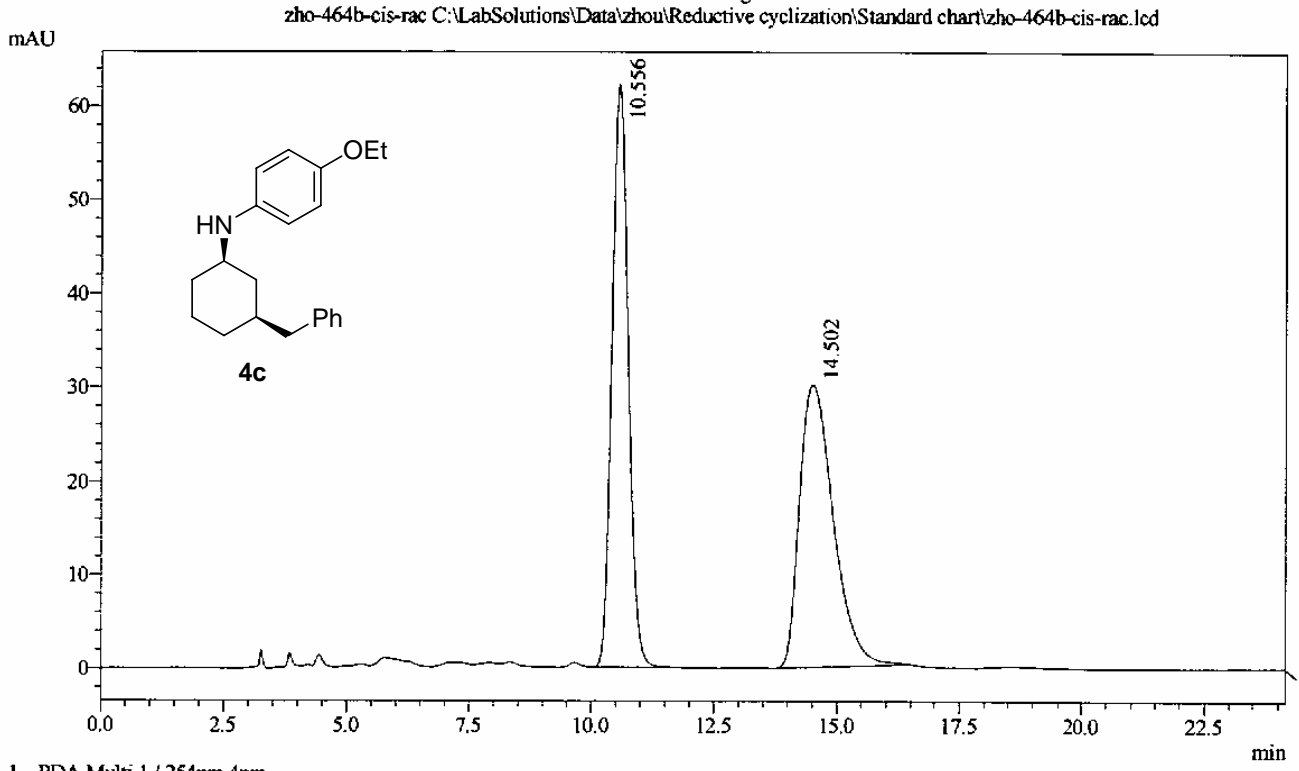

1 PDA Multi $1 / 254 \mathrm{~nm} 4 \mathrm{~nm}$

PeakTable

\begin{tabular}{|c|c|c|c|c|c|}
\hline \multicolumn{2}{|c|}{ PDA Ch1 254nm 4nm } & \multicolumn{4}{|c|}{ - } \\
\hline Peak\# & Ret. Time & As rea & Height & Area $\%$ & Height $\%$ \\
\hline 1 & 10.556 & 1463679 & 62171 & 50.367 & 67.347 \\
\hline 2 & 14.502 & 1442330 & 30143 & 49.633 & 32.653 \\
\hline Tota & & 2906009 & 92314 & 100.000 & 100.000 \\
\hline
\end{tabular}


Chromatogram

zho-468cis C:LabSolutions'Datazhou Reductive cyclization'Standard chartizho-468cis 1.Icd

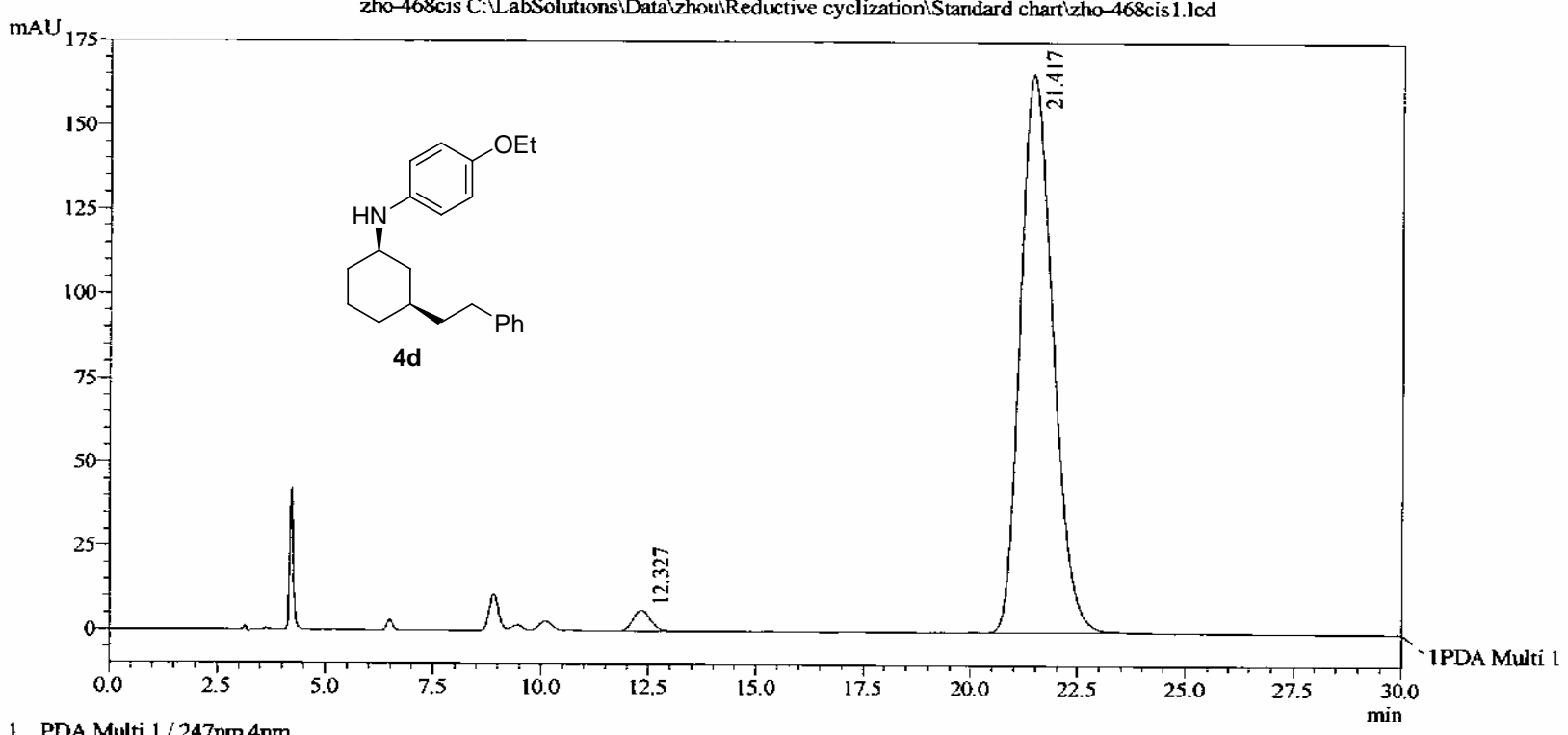

1 PDA Multi $1 / 247 \mathrm{~nm} 4 \mathrm{~nm}$

PeakTable

\begin{tabular}{|c|c|c|c|c|c|}
\hline Peak\# & Ret. Time & Area & Height & Area \% & Height \% \\
\hline-1 & 12.327 & 179301 & 6219 & 1994 & 3.617 \\
\hline 2 & 21.417 & 8810832 & 165723 & $98.0 \overline{06}$ & 96.383 \\
\hline Totail & & 8990132 & 171942 & 100.000 & 100.000 \\
\hline
\end{tabular}

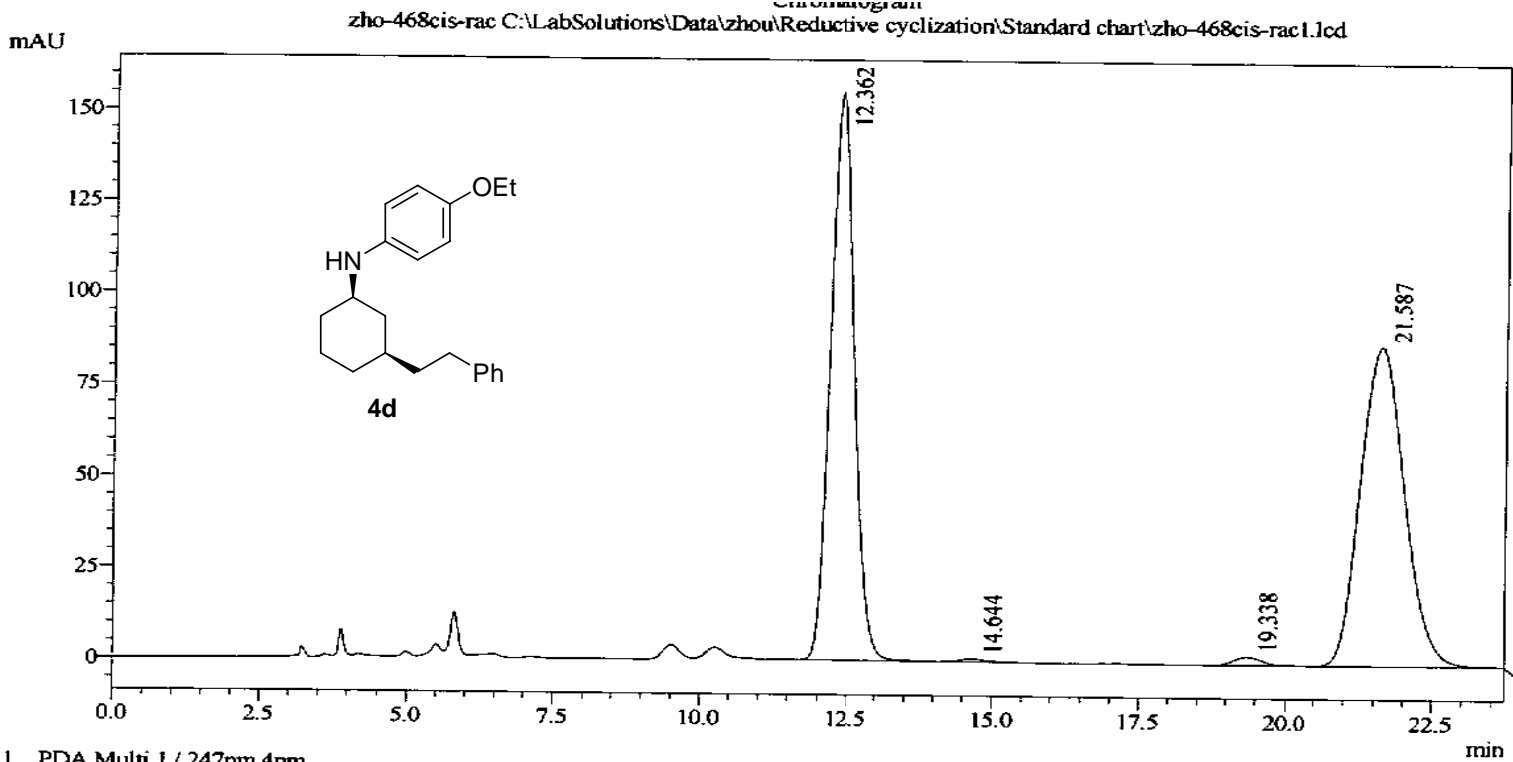

IPDA Multi 1

1 PDA Multi $1 / 247 \mathrm{~nm} 4 \mathrm{~nm}$

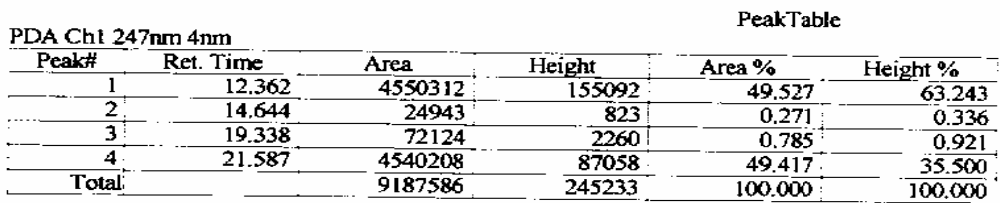




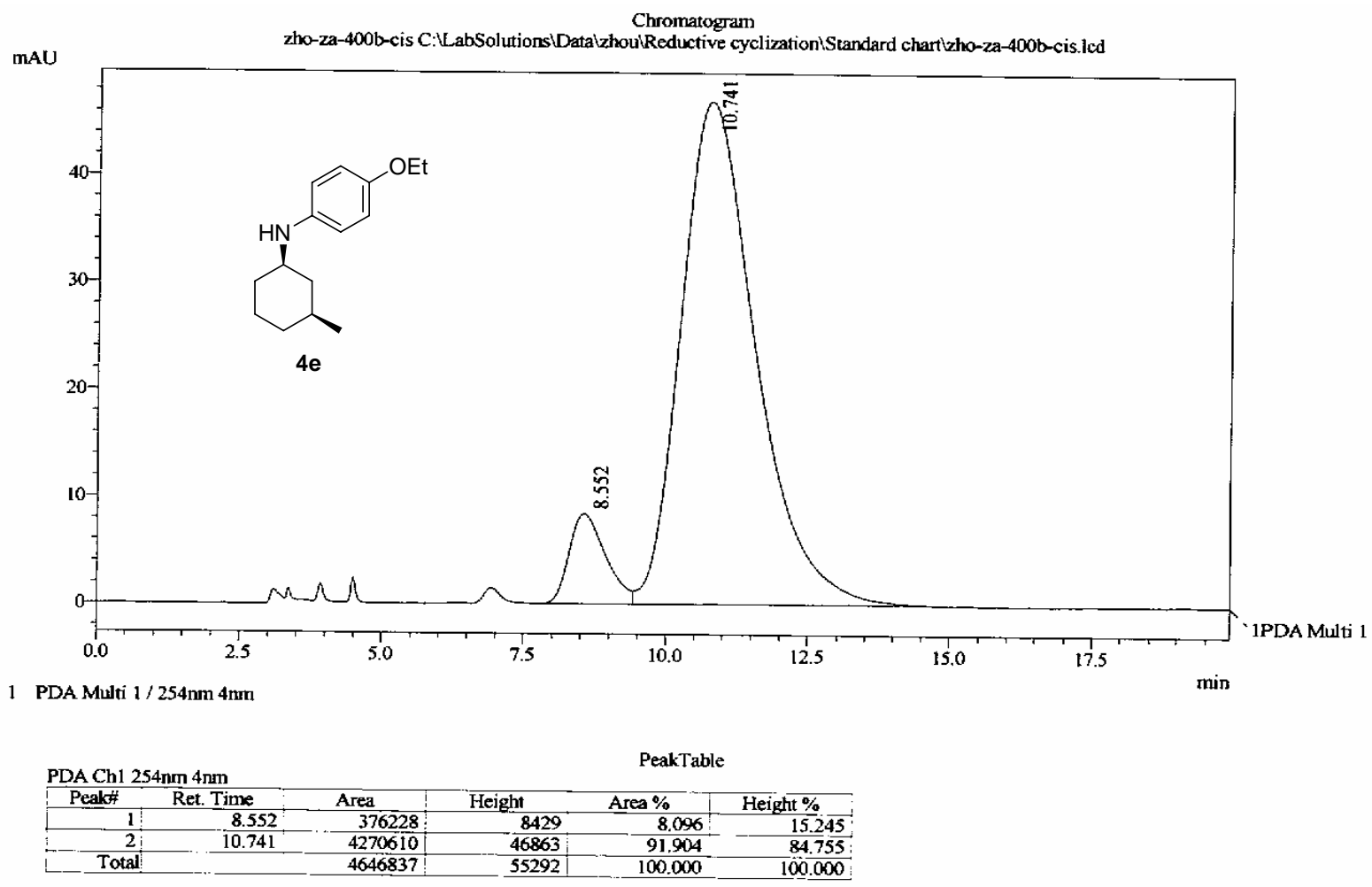

Chromatogram

mAU

zho-za-400b-cis-rac C:LabSolutionsLatalzhoulReductive cyclizationizho-za-400b-cis-rac.lcd

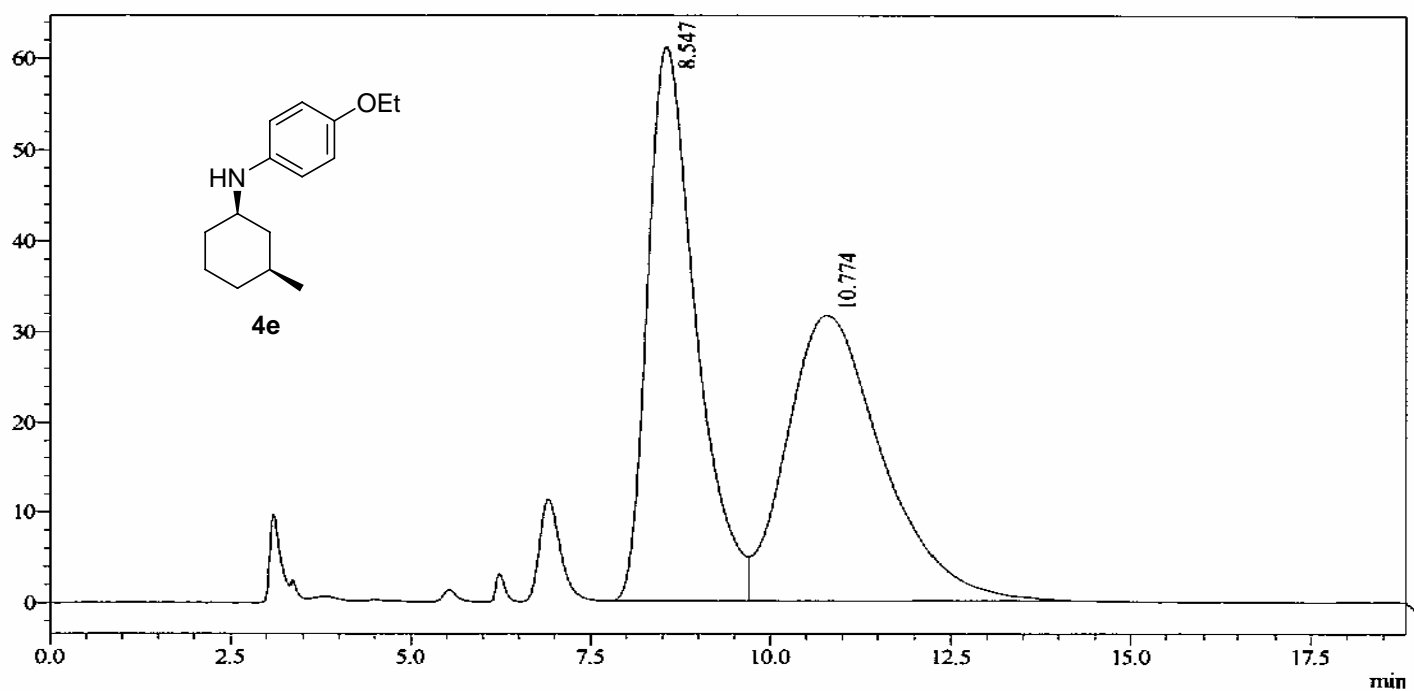

1 PDA Multi $1 / 247 \mathrm{~mm} 4 \mathrm{~nm}$

PeakTable

\begin{tabular}{|c|c|c|c|c|c|}
\hline Peak\# & Ret Time & Area & Height & Area \% & Height \% \\
\hline 1 & 8.547 & 2867697 & 61117 & 49.583 & $65.9 \mathrm{I} 1$ \\
\hline 2 & 10.774 & 2915936 & 31609 & 50.417 & 34.089 \\
\hline Total & & 5783633 & 92727 & 100.000 & 100.000 \\
\hline
\end{tabular}




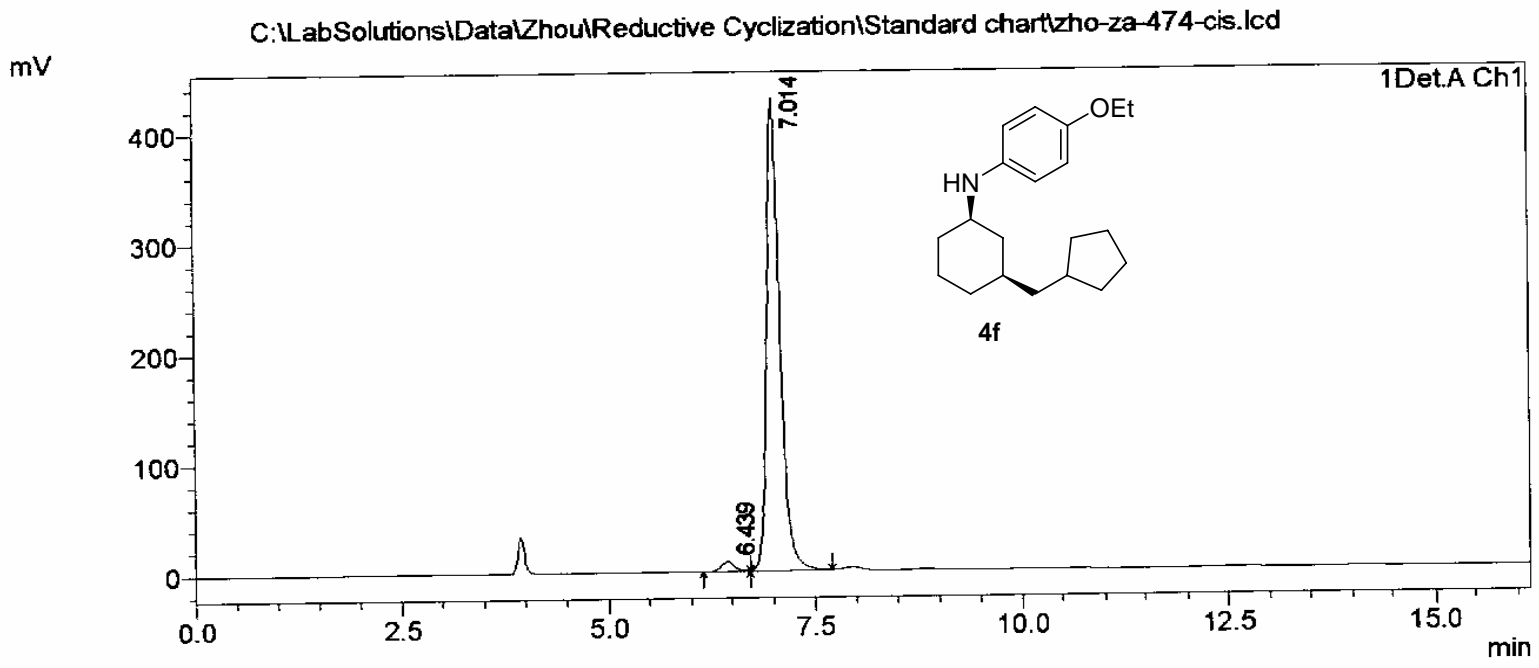

1 Det.A Ch1/247nm

\section{<Results>}

Detector A Ch1 $247 \mathrm{~nm}$
\begin{tabular}{|l|r|r|}
\hline Peak\# & Ret. Time & \multicolumn{1}{|c|}{ Area \% } \\
\hline 1 & 6.439 & 2.165 \\
\hline 2 & 7.014 & 97.835 \\
\hline Total & & 100.000 \\
\hline
\end{tabular}

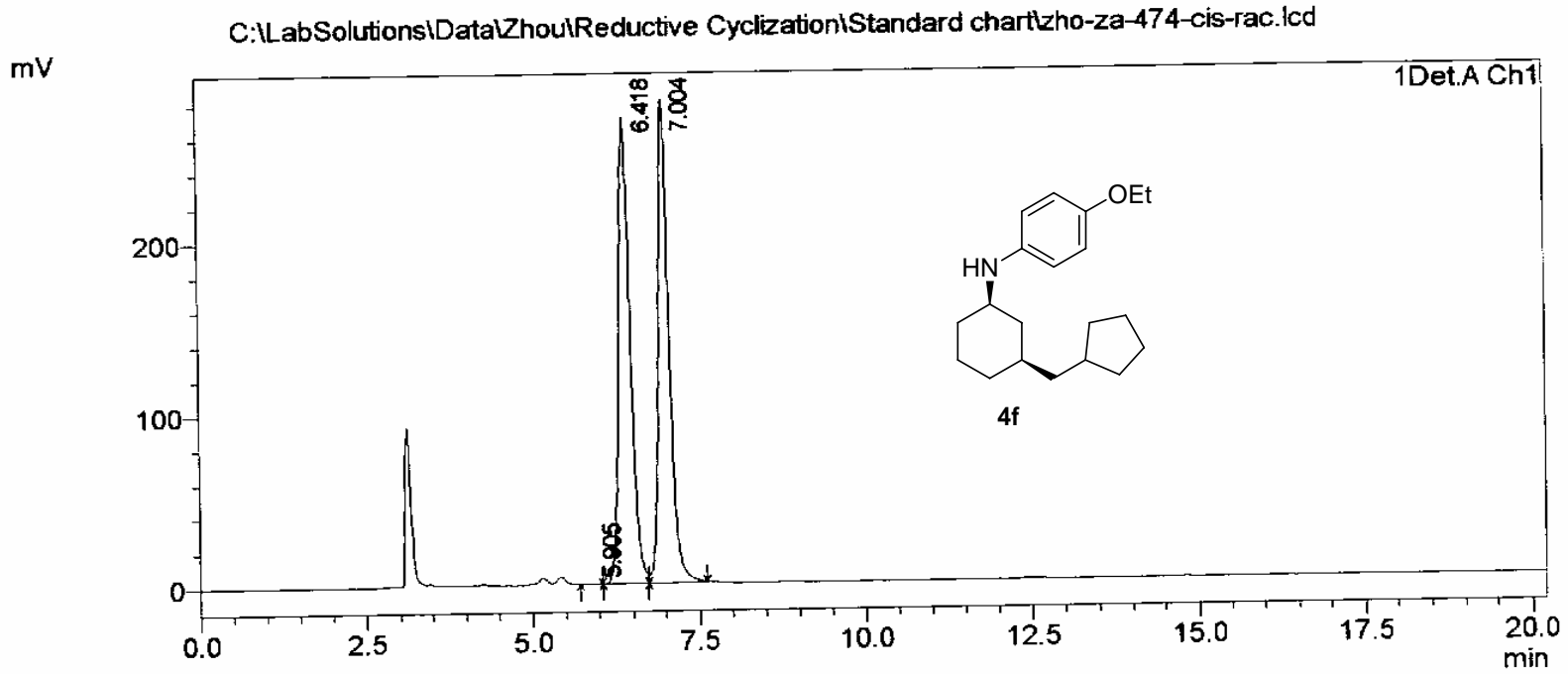

1 Det.A Ch $1 / 247 \mathrm{~nm}$

\section{<Results>}

Detector A Ch1 247nm

\begin{tabular}{|l|r|r|}
\hline Peak \# & Ret. Time & \multicolumn{1}{|c|}{ Area \% } \\
\hline 1 & 5.905 & 0.048 \\
\hline 2 & 6.418 & 49.692 \\
\hline 3 & 7.004 & 50.260 \\
\hline Total & & 100.000 \\
\hline
\end{tabular}


Chromatogram

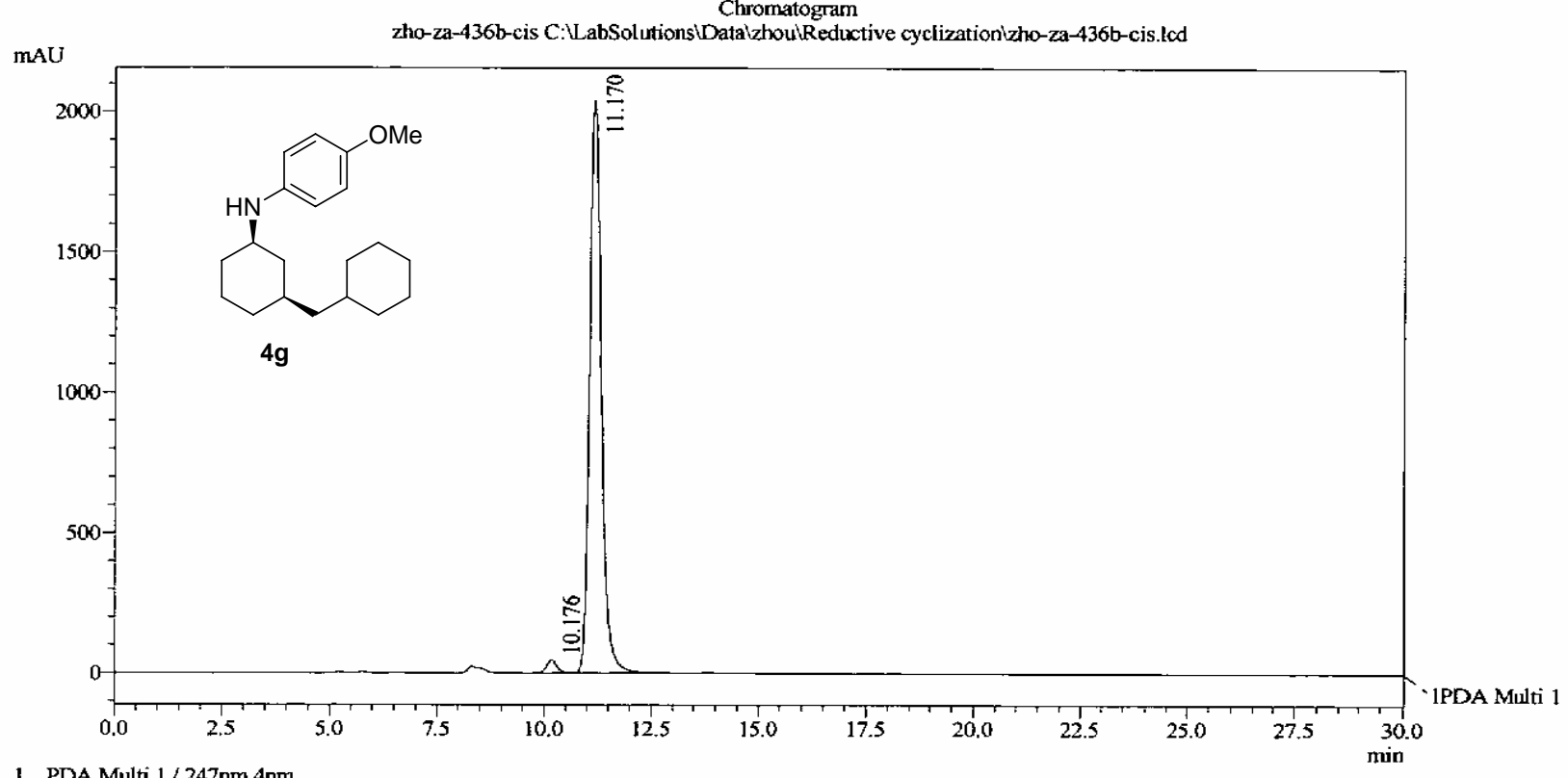

1 PDA Multi $1 / 247 \mathrm{~nm} 4 \mathrm{~nm}$

\begin{tabular}{|c|c|c|c|c|c|}
\hline \multicolumn{3}{|c|}{ PDA Chl 247nm 4nm } & \multicolumn{3}{|c|}{ PeakTable } \\
\hline Peak\# & Ret Time & Aren & Height & Aren \% & Height 0 \\
\hline 1 & 10.176 & 774840 & 46400 & 1.922 & 2.222 \\
\hline 2 & 11.170 & 39532331 & 2042182 & 98.078 & 97.778 \\
\hline Total & & 40307170 & 2088582 & 100.000 & 100.000 \\
\hline
\end{tabular}

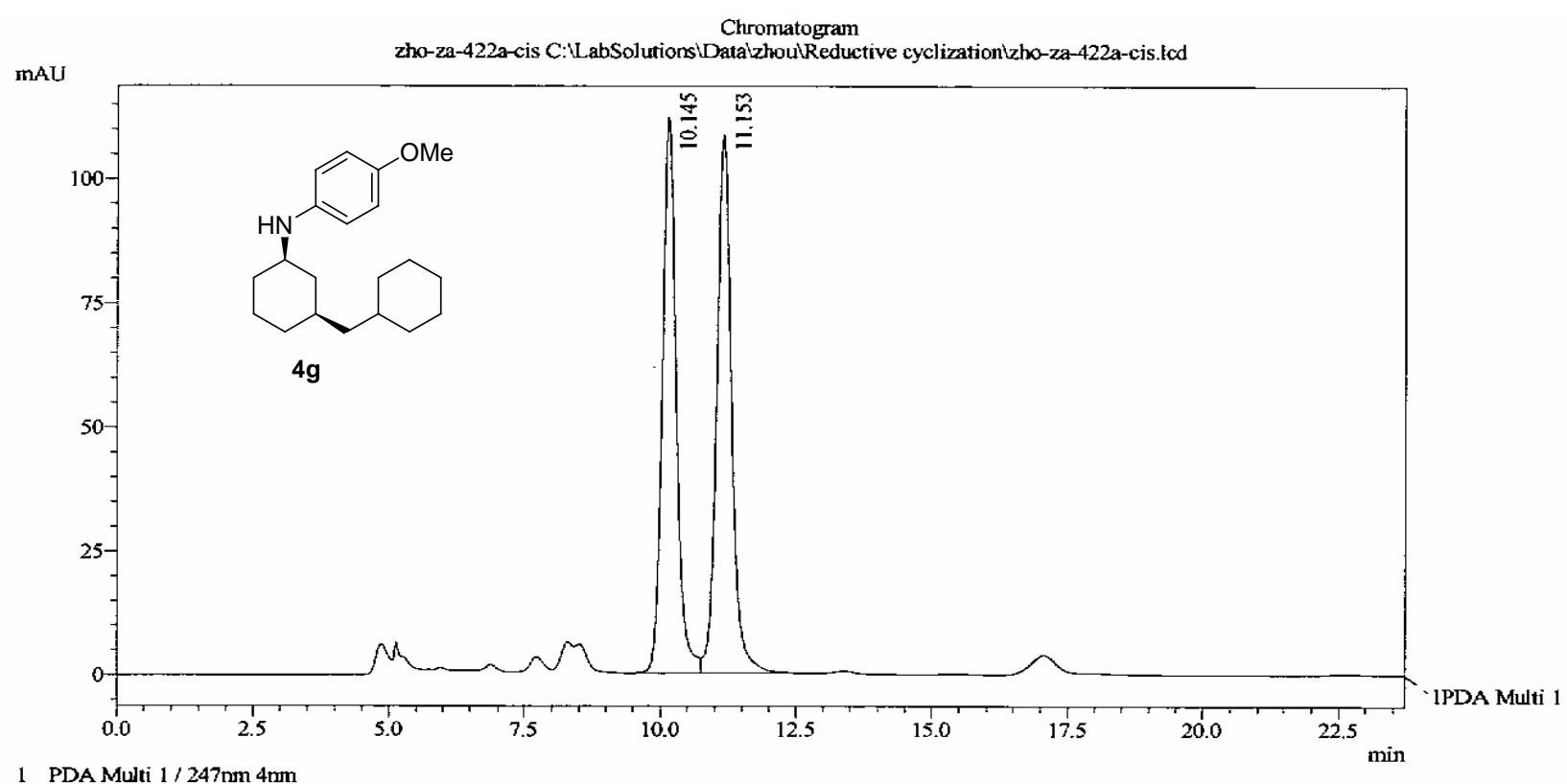

1 PDA Multi $1 / 247 \mathrm{~nm} 4 \mathrm{~mm}$

PeakTable

\begin{tabular}{|c|c|c|c|c|c|}
\hline \multicolumn{2}{|c|}{ PDA Ch1 247nm 4nm } & \multicolumn{3}{|r|}{ reak Iavie } & \multirow[b]{2}{*}{ Height \% } \\
\hline Peak\# & Ret. Time & Area & Height & Area \% & \\
\hline 1 & 10.145 & 203547 & 112036 & 49.815 & 50.810 \\
\hline 2 & 11.153 & 2050621 & 108464 & 50.185 & 49.190 \\
\hline Total & & 4086092 & 220501 & 100.000 & 100.000 \\
\hline
\end{tabular}


Chromatogram

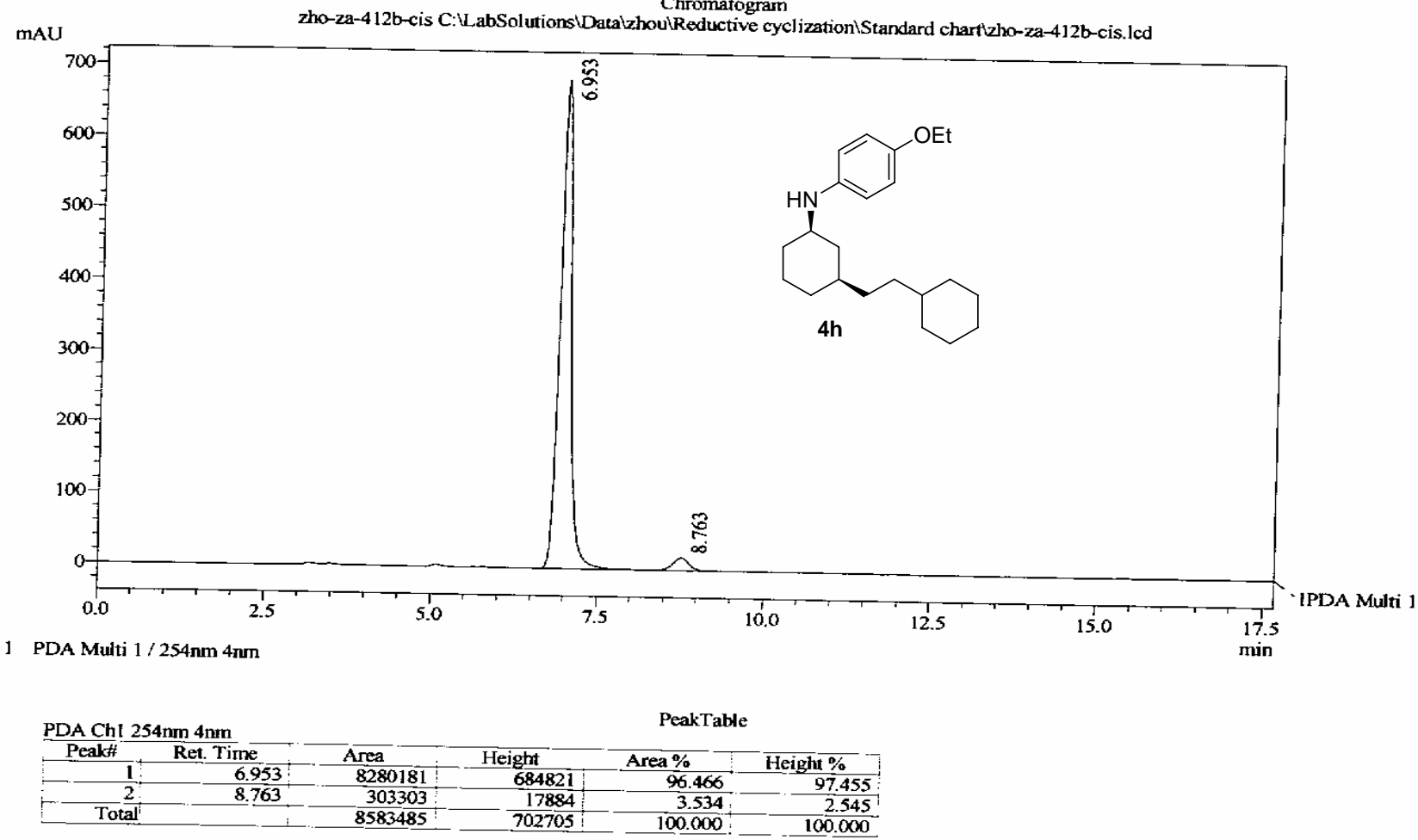

Chromatogram

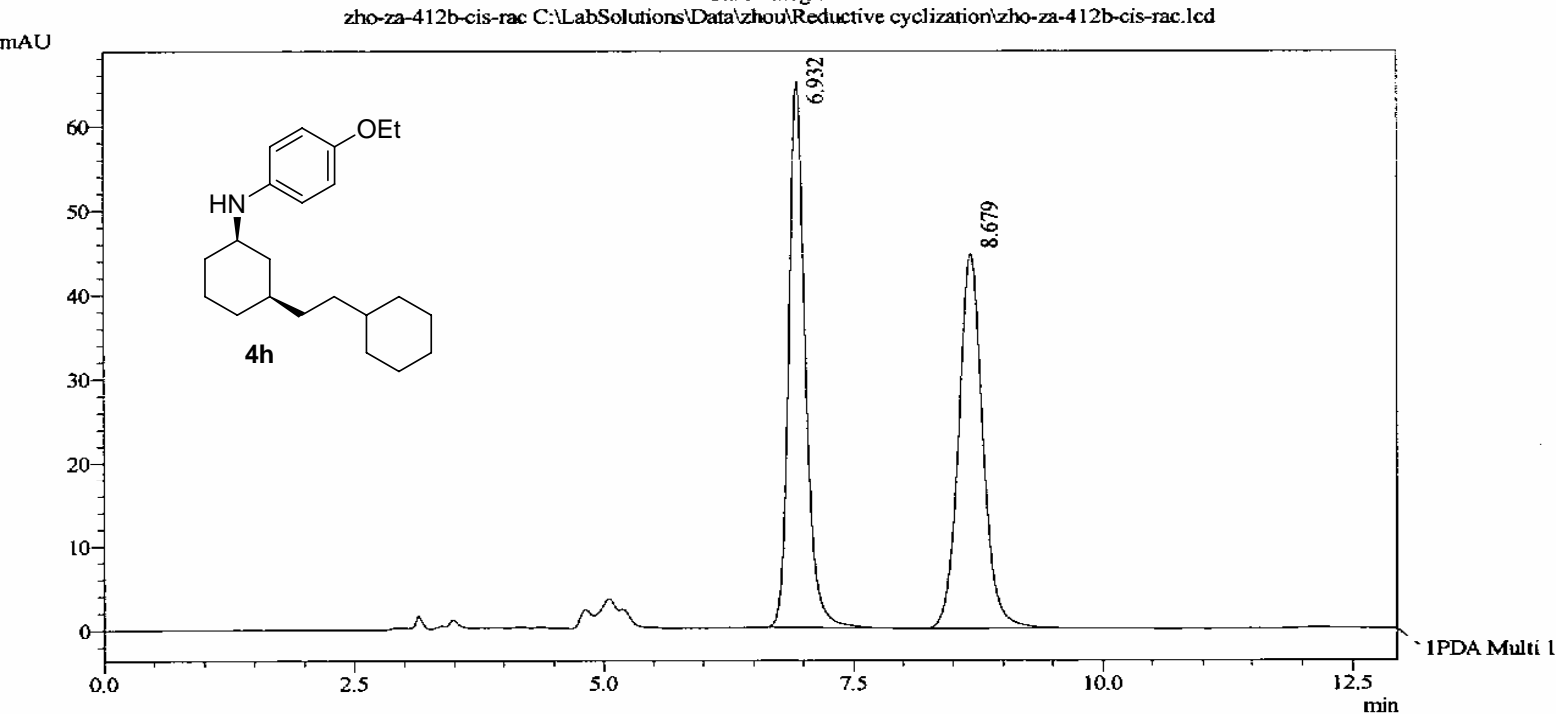

1 PDA Multi 1/254nm 4nm

PeakTable

\begin{tabular}{|c|c|c|c|c|c|}
\hline \multicolumn{6}{|c|}{ PDA Ch1 254nm 4nm } \\
\hline Peakł & Ret. Time & Area & Height & Area \% & Height \% \\
\hline 1 & 6.932 & 764153 & 64895 & 50.066 & 59.225 \\
\hline 2 & 8.679 & 762150 & 44678 & 49.934 & 40.775 \\
\hline Total & & 1526303 & 109573 & 100.000 & 100.000 \\
\hline
\end{tabular}


cmromatogram

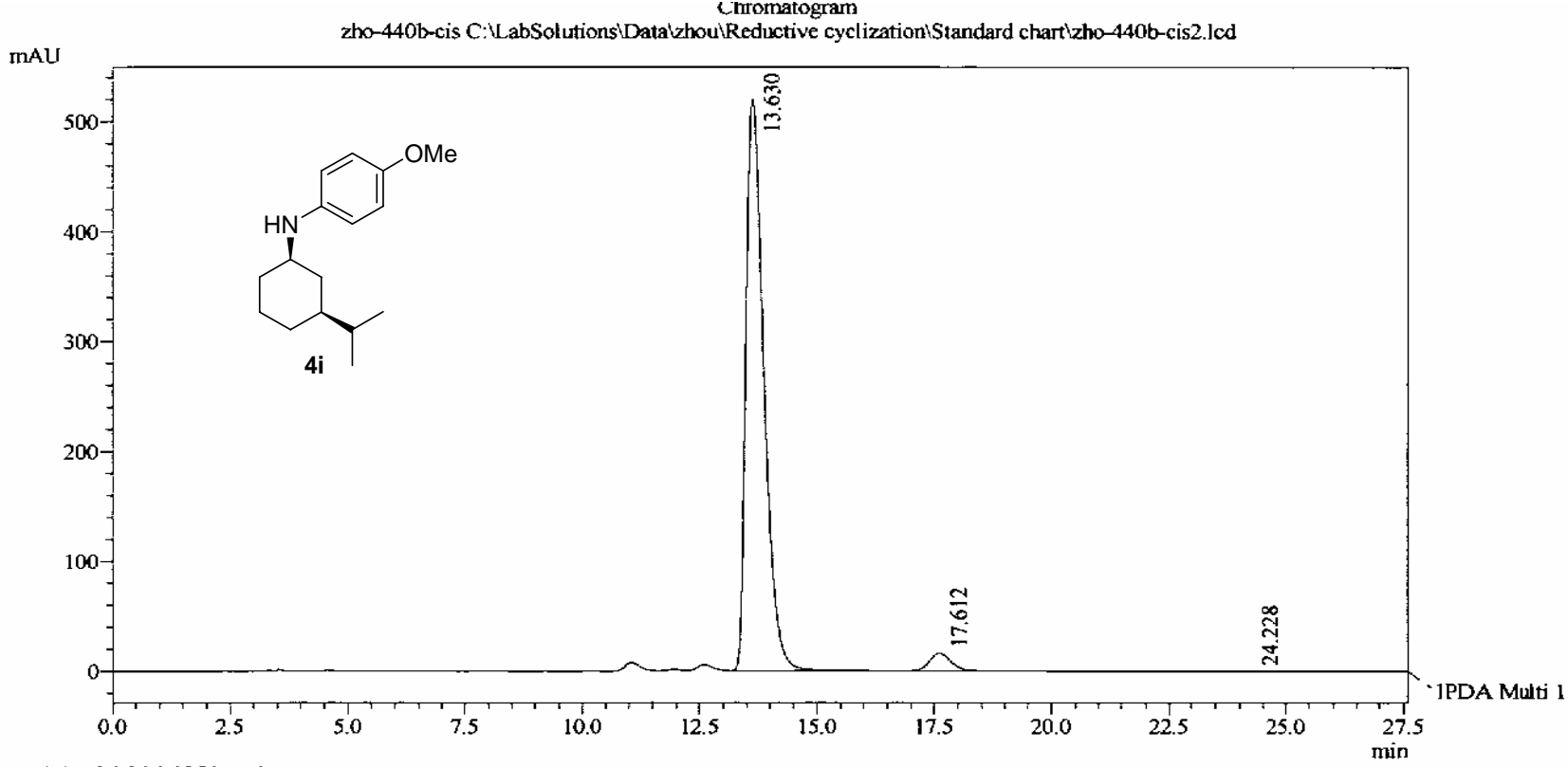

1 PDA Multi $1 / 254 \mathrm{~nm} 4 \mathrm{~nm}$

PeakTable

PDA Ch1 254nm 4nm

\begin{tabular}{|c|c|c|c|c|c|}
\hline Peak\# & Ret. Time & Area & Height & Area \% & Height \% \\
\hline 1 & 13.630 & 13982836 & 520298 & 96.351 & 96.899 \\
\hline 2 & 17.612 & 514332 & 16171 & 3.544 & 3.012 \\
\hline 3 & 24.228 & 15234 & 480 & 0.105 & 0.089 \\
\hline tal: & & 14512401 & 536948 & 100.000 & 100.000 \\
\hline
\end{tabular}

Chromatogram

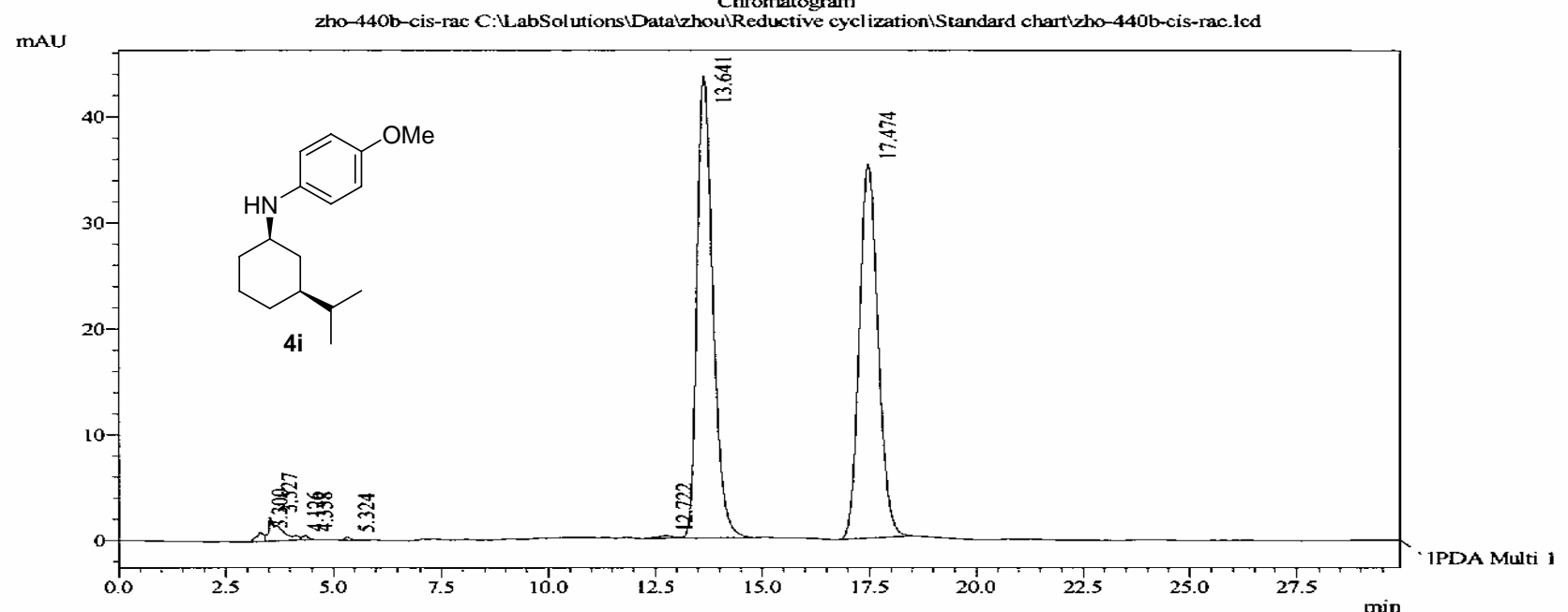

1 PDA Multi I / 254nm 4nm

PcakTable

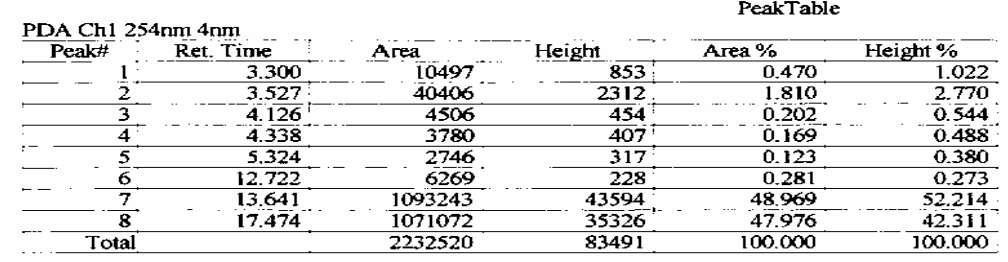


$\mathrm{mV}$

C:ILabSolutionsIDatalZhoulReductive CyclizationIStandard chartlzho-za-430b-cis.Icd

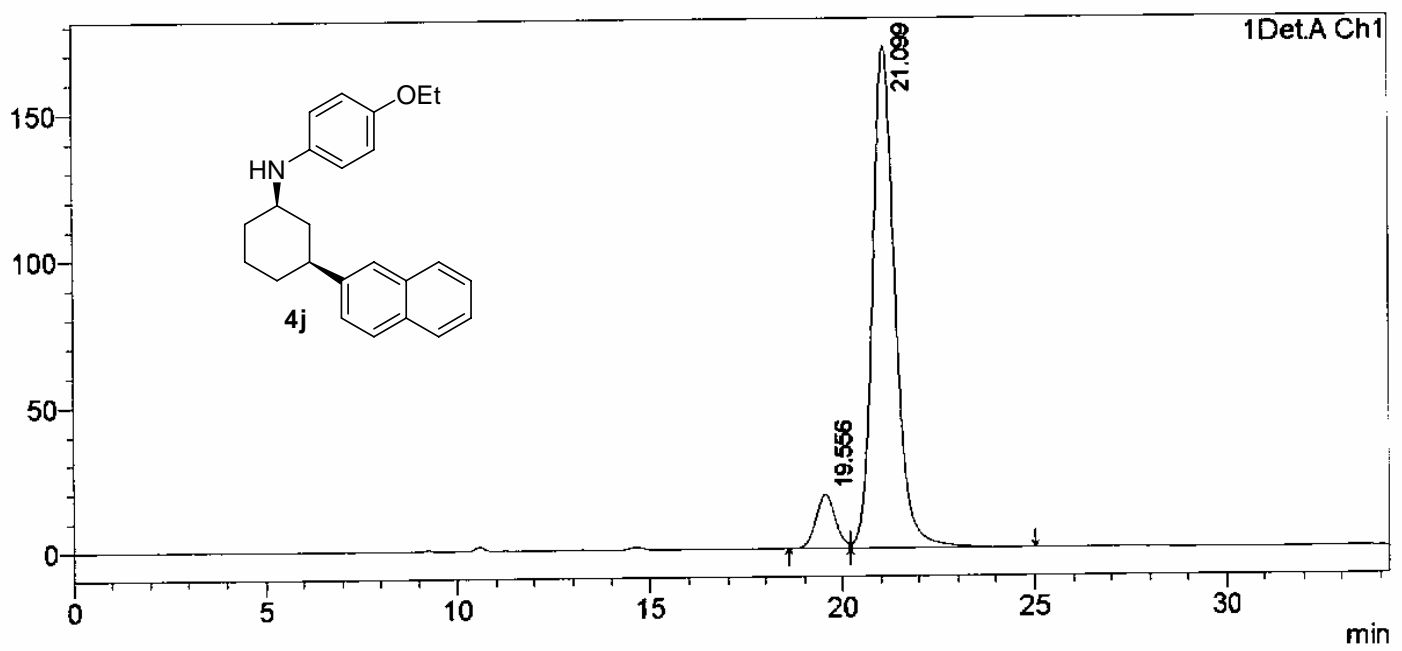

1 Det.A Ch1/247nm

<Results>

Detector A Ch1 $247 \mathrm{~nm}$
\begin{tabular}{|l|r|r|}
\hline Peak \# & Ret. Time & Area \% \\
\hline 1 & 19.556 & 8.757 \\
\hline 2 & 21.099 & 91.243 \\
\hline Total & & 100.000 \\
\hline
\end{tabular}

$\mathrm{mV}$

C:ILabSolutionsIDatalZhoulReductive Cyclizationizho-za-430c-cis-rac2.lcd

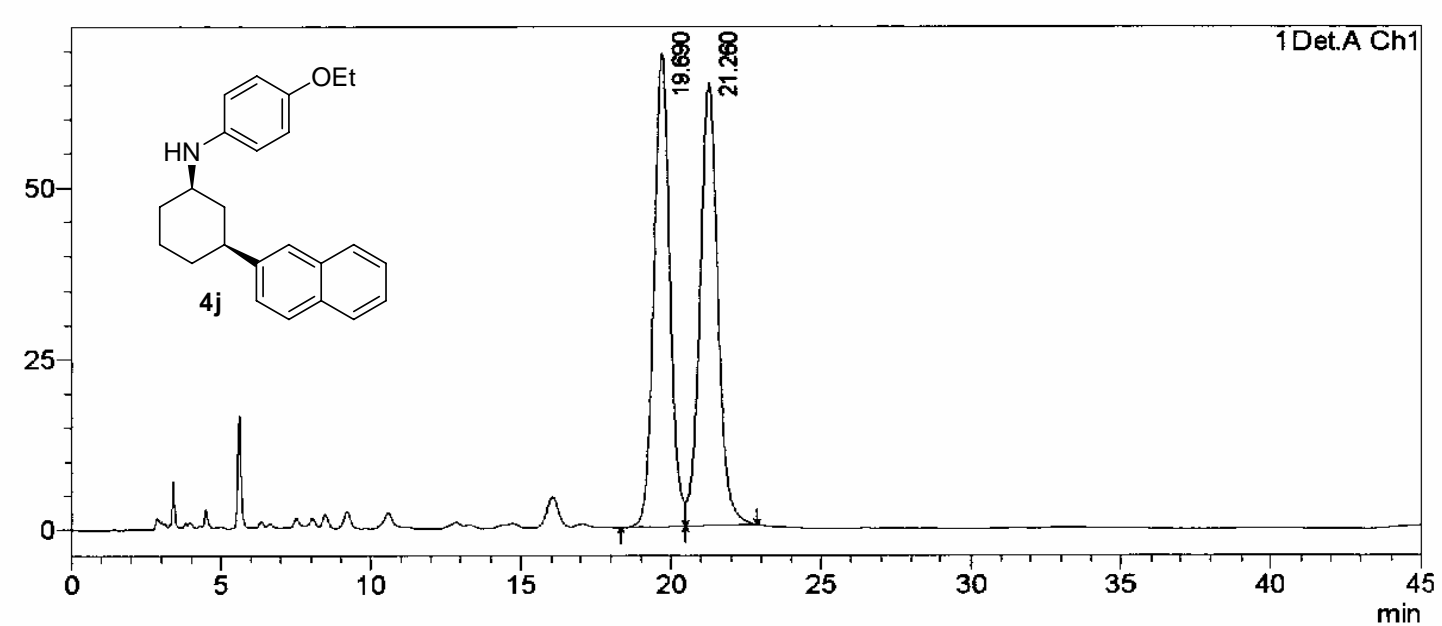

1 Det.A Ch1/247nm

<Results>

Detector A Ch1 247nm

\begin{tabular}{|l|r|r|}
\hline Peak \# & Ret. Time & \multicolumn{1}{c|}{ Area \% } \\
\hline 1 & 19.690 & 49.504 \\
\hline 2 & 21.260 & 50.496 \\
\hline Total & & 100.000 \\
\hline
\end{tabular}


Chromatogram

mAU

zho-zb-118-cis-ADH C:LabSolutions $\backslash$ Datalzhou Reductive cyclization $\backslash$ Standard chartizho-zb-118-cis-ADH.lcd

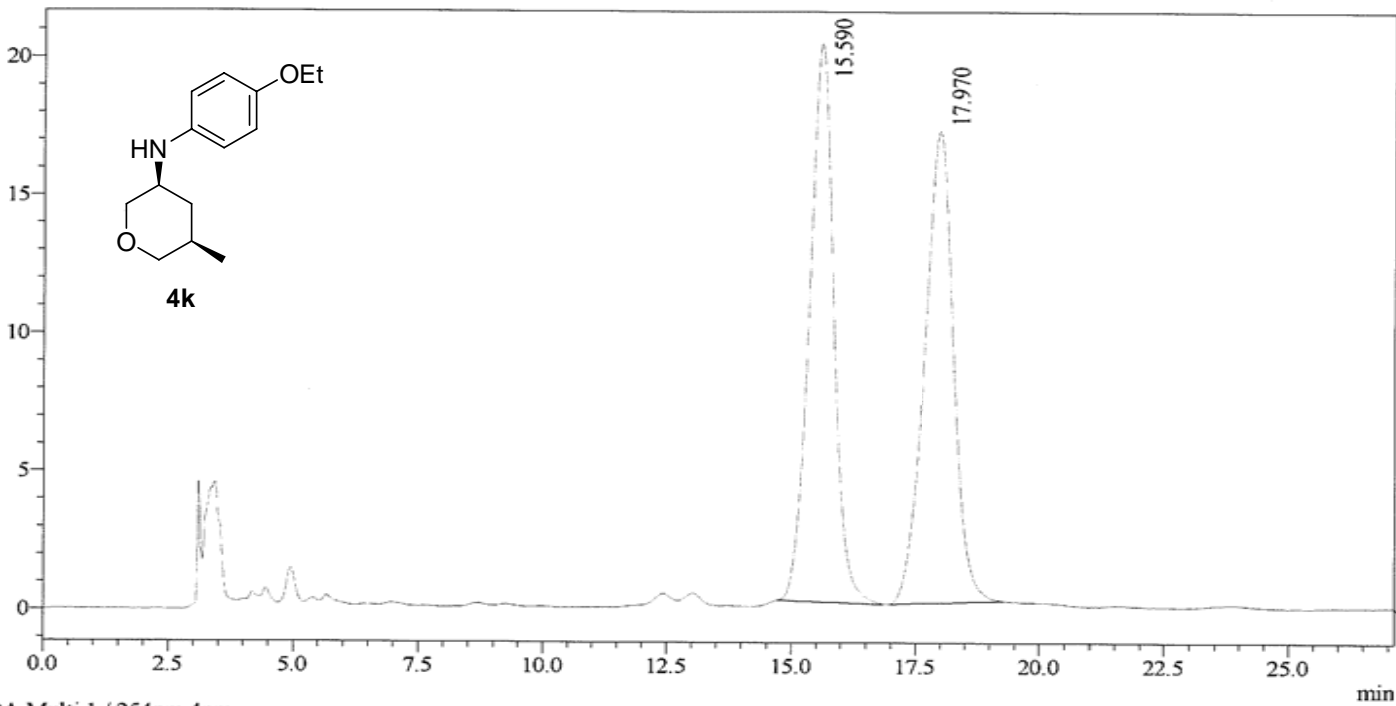

1 PDA Multi $1 / 254 \mathrm{~nm} 4 \mathrm{~nm}$

PDA Ch1 254nm 4nm

PeakTable

\begin{tabular}{|r|r|r|r|r|r|}
\hline \multicolumn{1}{|c|}{ Peak\# } & Ret. Time & \multicolumn{1}{c}{ Area } & Height & Area \% & \multicolumn{1}{c|}{ Height \% } \\
\hline 1 & 15.590 & 696035 & 20193 & 50.011 & 54.172 \\
\hline 2 & 17.970 & 695736 & 17083 & 49.989 & 45.828 \\
\hline Total & & 1391771 & 37276 & 100.000 & 100.000 \\
\hline
\end{tabular}

Chromatogran

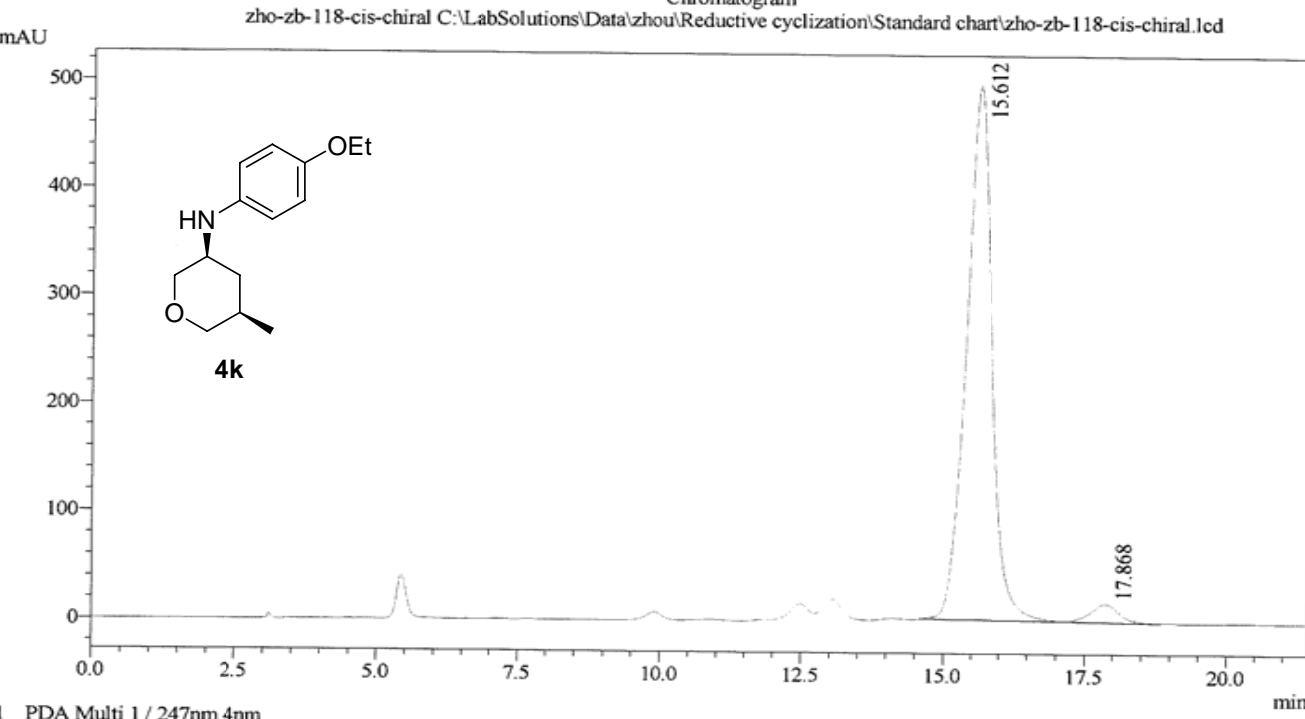

'PPDA Multi 1

1 PDA Multi $1 / 247 \mathrm{~nm} 4 \mathrm{~nm}$

PeakTable

PDA Ch1 $247 \mathrm{~nm} 4 \mathrm{~nm}$
\begin{tabular}{|r|r|r|r|r|r|}
\hline Peak\# & Ret. Time & \multicolumn{1}{|c|}{ Area } & Height & Area $\%$ & \multicolumn{1}{|c|}{ Height \% } \\
\hline 1 & 15.612 & 15690829 & 495325 & 96.421 & 96.697 \\
\hline 2 & 17.868 & 582482 & 16920 & 3.579 & 3.303 \\
\hline Total & & 16273311 & 512245 & 100.000 & 100.000 \\
\hline
\end{tabular}




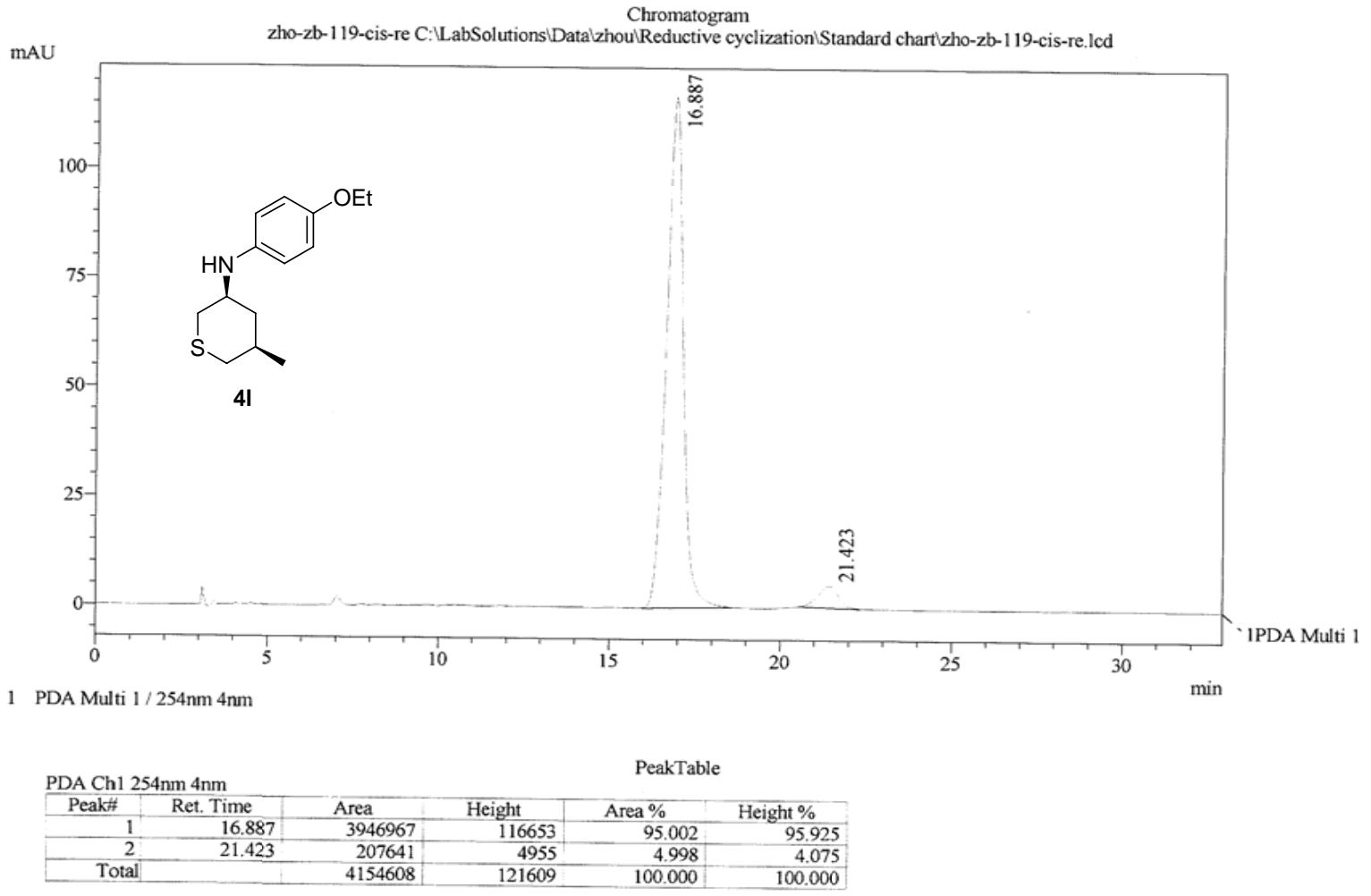

Chromatogram

mAU

zho-zb-119-cis-rac C: LabSolutions $\backslash$ Datalzhou'Reductive cyclization $\backslash$ Standard chart'zho-zb-119-cis-rac.lcd

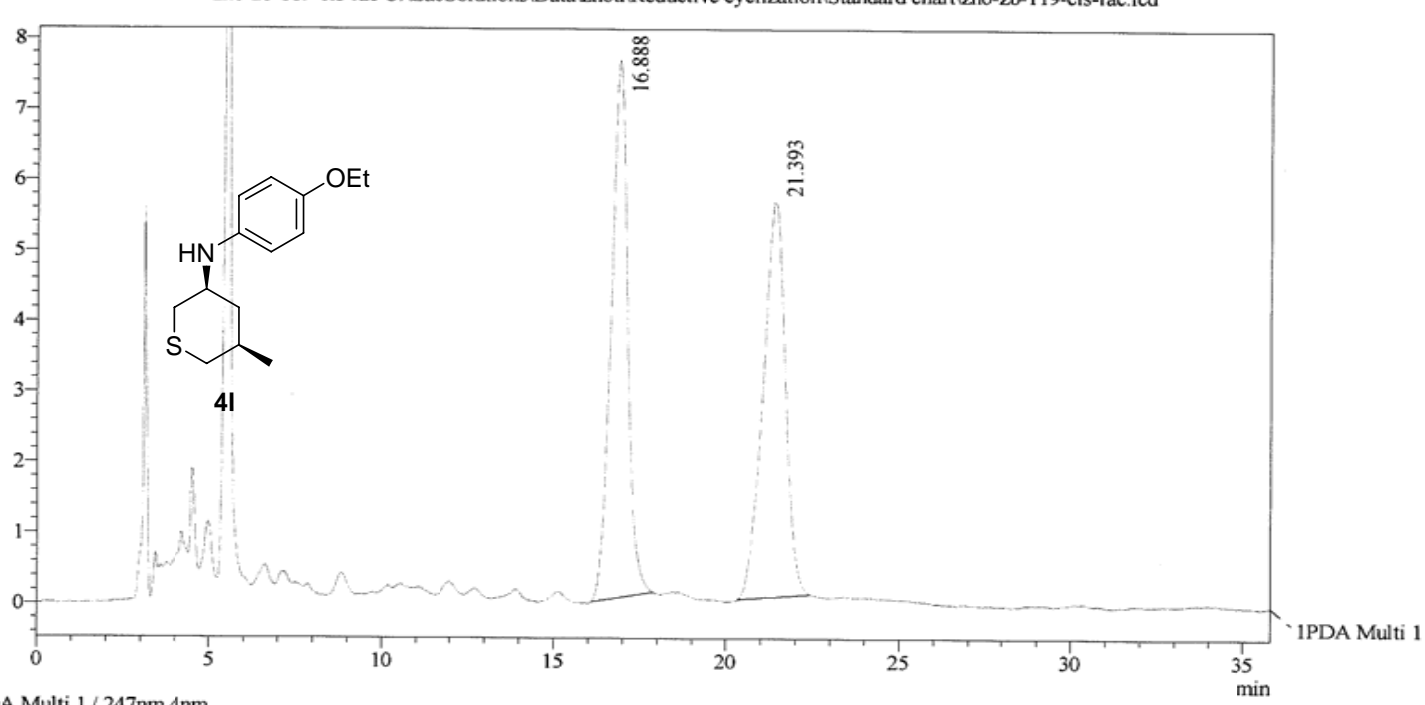

PeakTable

\begin{tabular}{|c|c|c|c|c|c|}
\hline Peak\# & Ret. Time & Area & Height & Area \% & Height \% \\
\hline 1 & 16.888 & 253029 & 7605 & 50.331 & 57.567 \\
\hline 2 & 21.393 & 249704 & 5606 & 49.669 & 42.433 \\
\hline Total & & 502732 & 13211 & 100.000 & 100.000 \\
\hline
\end{tabular}

